\title{
Palladium(II)-Catalyzed Reductive Cyclization of $N$-Tosyl-Tethered 1,7-Enynes: Enantioselective Synthesis of 1,2,3,4-Tetrahydroquinolines
}

\author{
Junjie Chen, Xiuling Han ${ }^{*}$ and Xiyan $\mathrm{Lu}^{*}$ \\ State Key Laboratory of Organometallic Chemistry, Shanghai Institute of Organic Chemistry, \\ University of Chinese Academy of Sciences, Chinese Academy of Sciences, 345 Lingling Road, \\ Shanghai 200032, China \\ Fax: (+86)-21-6416-6128; \\ Email: xlhan@mail.sioc.ac.cn; xylu@mail.sioc.ac.cn
}

\section{Supporting Information}

\section{Table of Contents}

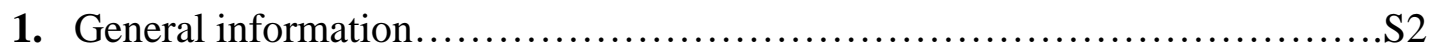

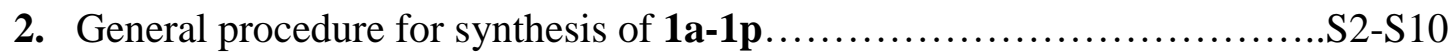

3. General procedure for $\mathrm{Pd}\left(\mathrm{CH}_{3} \mathrm{CN}\right)_{4}\left(\mathrm{BF}_{4}\right)_{2} /(\mathrm{R})-\mathrm{SEGPHOS}$ catalyzed cyclization of

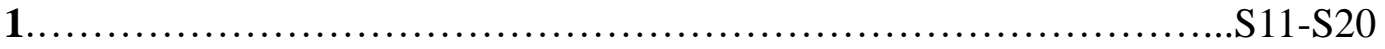

4. Procedure for asymmetric cyclization of $\mathbf{1 a}$ in $2 \mathrm{mmoL}$ scale................S21

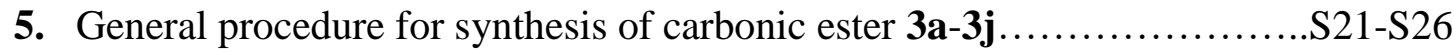

6. General procedure for $\mathrm{Pd}\left(\mathrm{CH}_{3} \mathrm{CN}\right)_{4}\left(\mathrm{BF}_{4}\right)_{2} /(\mathrm{R})-\mathrm{SEGPHOS}$ catalyzed cyclization of 3. S27-S33

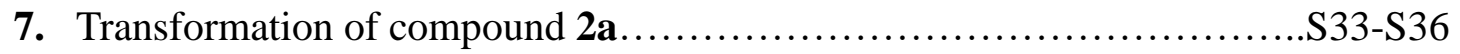

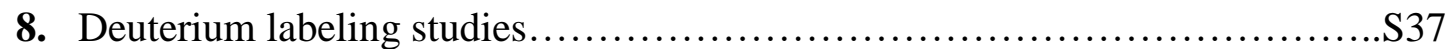

9. Copies of ${ }^{1} \mathrm{H}$ NMR, ${ }^{13} \mathrm{C}$ NMR and ${ }^{19} \mathrm{~F}$ NMR spectra........................... 3382

10. HPLC analysis reports of chiral products.........................S103-S132

11. Crystal structure analyses for product $8 \mathbf{8} \ldots \ldots \ldots \ldots \ldots \ldots \ldots \ldots \ldots . . . \ldots 133-\mathrm{S} 134$ 


\section{General information}

All solvents were dried and distilled using standard procedures. Unless otherwise noted, reagents were obtained from commercial sources and used without further purification. ${ }^{1} \mathrm{H}$ NMR and ${ }^{13} \mathrm{C}$ NMR were recorded in deuterated chloroform $\left(\mathrm{CDCl}_{3}\right)$. Coupling constants are recorded in hertz, and chemical shifts are recorded as $\delta$ values in ppm. The following abbreviations are used to describe multiplicities: $\mathrm{s}=$ singlet, $\mathrm{d}=$ doublet, $\mathrm{dd}=$ double doublet, $\mathrm{t}=$ triplet, $\mathrm{m}=$ multiplet. High-resolution mass spectra were carried out on a mass spectrometer with a TOF analyzer (ESI). Infrared spectra were recorded on a FT-IR spectrometer. Melting points were determined by using a local hot-stage melting point apparatus and are uncorrected. The optical rotation was measured on a Perkin-Elmer 341 polarimeter and the enantiomeric excesses were determined after separation of the enantiomers by HPLC on a Perkin-Elmer (785A, 200 IC Pump) or Waters (515 Pump, 2487. Dual Absorbance Detector) instruments. For column chromatography, silica gel of 200-300 mesh size was used.

\section{General procedure for synthesis of 1a-1p}

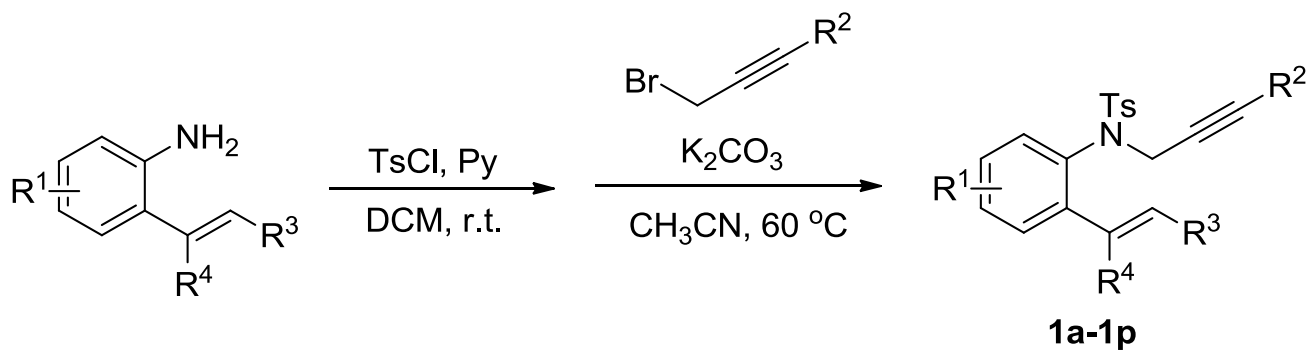

In a $50 \mathrm{~mL}$ single neck flask, the corresponding aniline (2.0 mmol, 1.0 equiv.) was dissolved in anhydrous DCM (25 mL). Then TsCl (2.4 mmol, 1.2 equiv.) and pyridine (3.0 mmol, 1.5 equiv.) were added to the mixture and the resulting solution was stirred at room temperature. After completion of the reaction, the crude reaction mixture was quenched by $\mathrm{HCl}(10 \mathrm{~mL}, 1 \mathrm{M})$, extracted with ethyl acetate $(15 \mathrm{~mL} * 3)$. The organic layer was dried over anhydrous $\mathrm{Na}_{2} \mathrm{SO}_{4}$, and then concentrated under reduced pressure. This crude residue was dissolved in $\mathrm{CH}_{3} \mathrm{CN}(20 \mathrm{~mL})$, then $\mathrm{K}_{2} \mathrm{CO}_{3}(2.4 \mathrm{mmol}$, 1.2 equiv.) and propargyl bromide ( $3.0 \mathrm{mmol}, 1.5$ equiv.) were added sequentially. 
The mixture was stirred at $60{ }^{\circ} \mathrm{C}$ for $2 \mathrm{~h}$, after which time TLC indicated complete conversion. The mixture was filtered by celite, washed with ethyl acetate. The solvent was removed under reduced pressure and the residue was purified by flash column chromatography (petroleum ether : ethyl acetate $=5: 1$ ) to obtain the compound 1 .<smiles>[3H]N(CC#CC#CCC(=O)OC)c1ccccc1C(C)=O</smiles>

$1 \mathrm{a}$

White solid; m.p.: $72-73{ }^{\circ} \mathrm{C}$; (628 mg; $69 \%$ yield for two steps); ${ }^{1} \mathbf{H}$ NMR (400 MHz, $\left.\mathbf{C D C l}_{3}\right): \delta 7.68(\mathrm{~d}, J=8.4 \mathrm{~Hz}, 2 \mathrm{H}), 7.35-7.18(\mathrm{~m}, 5 \mathrm{H}), 6.96(\mathrm{~d}, J=8.0 \mathrm{~Hz}, 1 \mathrm{H}), 5.94$ (q, $J=1.2 \mathrm{~Hz}, 1 \mathrm{H}), 4.58(\mathrm{br}, 1 \mathrm{H}), 4.14(\mathrm{br}, 1 \mathrm{H}), 3.74(\mathrm{~s}, 3 \mathrm{H}), 2.59$ (d, $J=0.8 \mathrm{~Hz}, 3 \mathrm{H})$, 2.44 (s, 3H), -0.03 (s, 9H); ${ }^{\mathbf{1 3}} \mathbf{C}$ NMR (100 MHz, $\left.\mathbf{C D C l}_{3}\right): \delta$ 166.8, 155.9, 145.1, 143.9, 136.1, 135.9, 129.4, 129.1, 128.9, 128.8, 128.5, 128.1, 120.0, 99.0, 91.6, 51.1, 42.3, 21.6, 20.5, -0.6; IR (neat, $\mathbf{~ c m}^{-1}$ ): $v$ 2964, 2166, 1708, 1632, 1483, 1346, 1161, 1086, 982, 843, 762; HRMS calculated for $\mathrm{C}_{24} \mathrm{H}_{33} \mathrm{~N}_{2} \mathrm{O}_{4} \mathrm{SSi}\left(\mathrm{M}+\mathrm{NH}_{4}\right)^{+}$: 473.1925; Found: 473.1909.

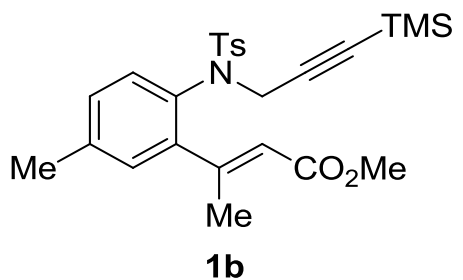

White solid; m.p.: $71-72{ }^{\circ} \mathrm{C}$; $\left(610 \mathrm{mg}\right.$; $65 \%$ yield for two steps); ${ }^{1} \mathbf{H}$ NMR (400 MHz, $\left.\mathbf{C D C l}_{3}\right): \delta 7.68(\mathrm{~d}, J=8.4 \mathrm{~Hz}, 2 \mathrm{H}), 7.28(\mathrm{~d}, J=8.4 \mathrm{~Hz}, 2 \mathrm{H}), 7.04-6.99(\mathrm{~m}, 2 \mathrm{H}), 6.84$ (d, $J=8.4 \mathrm{~Hz}, 1 \mathrm{H}), 5.90$ (q, $J=1.2 \mathrm{~Hz}, 1 \mathrm{H}), 4.59$ (br, 1H), 4.08 (br, 1H), 3.74 (s, 3H), $2.57(\mathrm{~d}, J=1.2 \mathrm{~Hz}, 3 \mathrm{H}), 2.44(\mathrm{~s}, 3 \mathrm{H}), 2.34(\mathrm{~s}, 3 \mathrm{H}),-0.02(\mathrm{~s}, 9 \mathrm{H}) ;{ }^{13} \mathbf{C}$ NMR (100 MHz, $\left.\mathbf{C D C l}_{3}\right): \delta 166.8,156.2,144.9,143.8,138.9,136.3,133.3,129.8,129.4,128.8$, 128.6, 119.8, 99.3, 91.5, 51.1, 42.4, 21.6, 21.1, 20.6, -0.5; IR (neat, $\mathbf{c m}^{-1}$ ): $v 2952$, 2173, 1715, 1645, 1492, 1345, 1248, 1202, 1156, 990, 841, 663; HRMS calculated for $\mathrm{C}_{25} \mathrm{H}_{35} \mathrm{~N}_{2} \mathrm{O}_{4} \mathrm{SSi}\left(\mathrm{M}+\mathrm{NH}_{4}\right)^{+}: 487.2081$; Found: 487.2068. 


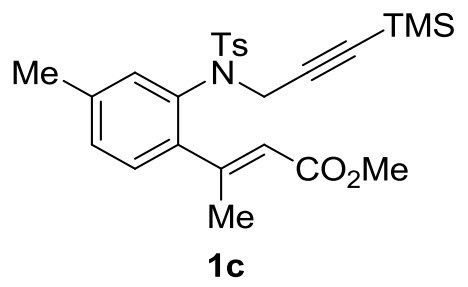

White solid; m.p.: $95-96{ }^{\circ} \mathrm{C}$; $\left(629 \mathrm{mg}\right.$; $67 \%$ yield for two steps); ${ }^{1} \mathbf{H}$ NMR (400 MHz, $\left.\mathbf{C D C l}_{3}\right): \delta 7.69(\mathrm{~d}, J=8.0 \mathrm{~Hz}, 2 \mathrm{H}), 7.29(\mathrm{~d}, J=8.4 \mathrm{~Hz}, 2 \mathrm{H}), 7.16-7.11(\mathrm{~m}, 2 \mathrm{H}), 6.81$ (s, 1H), 5.89 (q, $J=1.2 \mathrm{~Hz}, 1 \mathrm{H}), 4.59$ (br, 1H), 4.08 (br, 1H), 3.74 (s, 3H), 2.55 (d, J $=1.2 \mathrm{~Hz}, 3 \mathrm{H}), 2.45(\mathrm{~s}, 3 \mathrm{H}), 2.23(\mathrm{~s}, 3 \mathrm{H}),-0.01$ (s, 9H); ${ }^{13} \mathbf{C}$ NMR (100 MHz, CDCl $)$ : $\delta 166.9,156.1,143.8,142.1,138.1,136.3,135.8,129.8,129.6,129.3,129.0,128.7$, 119.8, 99.2, 91.5, 51.1, 42.4, 21.6, 20.9, 20.6, -0.5; IR (neat, $\mathbf{c m}^{-1}$ ): v 2948, 2185, 1713, 1644, 1346, 1251, 1159, 1086, 1062, 838, 657; HRMS calculated for $\mathrm{C}_{25} \mathrm{H}_{35} \mathrm{~N}_{2} \mathrm{O}_{4} \mathrm{SSi}\left(\mathrm{M}+\mathrm{NH}_{4}\right)^{+}$: 487.2081; Found: 487.2068.<smiles>COc1ccc([N-]C#CC(C)(C)C)c(/C(C)=C/C(C)=O)c1</smiles>

White solid; m.p.: $92-93{ }^{\circ} \mathrm{C}$; (602 mg; $62 \%$ yield for two steps); ${ }^{1} \mathbf{H}$ NMR (400 MHz, $\left.\mathbf{C D C l}_{3}\right): \delta 7.66(\mathrm{~d}, J=8.4 \mathrm{~Hz}, 2 \mathrm{H}), 7.27(\mathrm{~d}, J=8.4 \mathrm{~Hz}, 2 \mathrm{H}), 6.86(\mathrm{~d}, J=8.8 \mathrm{~Hz}, 1 \mathrm{H})$, 6.73-6.70 (m, 2H), 5.89 (q, $J=1.6 \mathrm{~Hz}, 1 \mathrm{H}), 4.60$ (d, $J=19.2 \mathrm{~Hz}, 1 \mathrm{H}), 4.60$ (d, $J=$ $18.0 \mathrm{~Hz}, 1 \mathrm{H}), 3.78$ (s, 3H), 3.73 (s, 3H), 2.55 (d, J = $1.2 \mathrm{~Hz}, 3 \mathrm{H}), 2.42$ (s, 3H), -0.02 (s, 9H); ${ }^{13} \mathbf{C}$ NMR (100 MHz, $\left.\mathbf{C D C l}_{3}\right): \delta$ 166.8, 159.4, 155.9, 146.5, 143.8, 136.3, 130.3, 129.4, 128.6, 128.5, 119.9, 114.2, 113.3, 99.4, 91.5, 55.5, 51.1, 42.6, 21.6, 20.6, -0.4; IR (neat, $\mathbf{c m}^{-\mathbf{1}}$ ): v 2956, 2180, 1713, 1642, 1344, 1220, 1160, 1086, 996, 843, 663; HRMS calculated for $\mathrm{C}_{25} \mathrm{H}_{35} \mathrm{~N}_{2} \mathrm{O}_{5} \mathrm{SSi}\left(\mathrm{M}+\mathrm{NH}_{4}\right)^{+}$: 503.2030; Found: 503.2018. 


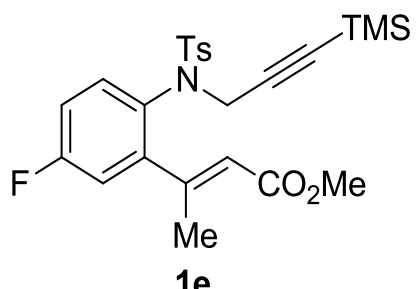

White solid; m.p.: $86-87^{\circ} \mathrm{C}$; (558 mg; $59 \%$ yield for two steps); ${ }^{1} \mathbf{H}$ NMR (400 MHz, $\left.\mathbf{C D C l}_{3}\right): \delta 7.64(\mathrm{~d}, J=8.0 \mathrm{~Hz}, 2 \mathrm{H}), 7.28(\mathrm{~d}, J=8.0 \mathrm{~Hz}, 2 \mathrm{H}), 6.96-6.87(\mathrm{~m}, 3 \mathrm{H}), 5.91$ (q, $J=1.2 \mathrm{~Hz}, 1 \mathrm{H}), 4.54(\mathrm{br}, 1 \mathrm{H}), 4.09(\mathrm{br}, 1 \mathrm{H}), 3.73(\mathrm{~s}, 3 \mathrm{H}), 2.54(\mathrm{~d}, J=1.2 \mathrm{~Hz}, 3 \mathrm{H})$, $2.43(\mathrm{~s}, 3 \mathrm{H}),-0.03(\mathrm{~s}, 9 \mathrm{H}) ;{ }^{13} \mathbf{C}$ NMR (100 MHz, $\left.\mathbf{C D C l}_{3}\right): \delta 166.9,162.0(\mathrm{~d}, J=$ 248.2 Hz), 154.4, 147.5 (d, $J=7.6 \mathrm{~Hz}), 144.1,135.8,131.9$ (d, $J=3.1 \mathrm{~Hz}), 130.9$ (d, $J=9.1 \mathrm{~Hz}), 129.6,128.6,120.6,116.1(\mathrm{~d}, J=22.8 \mathrm{~Hz}), 114.9(\mathrm{~d}, J=22.7 \mathrm{~Hz}), 98.9$, 92.0, 51.2 42.5, 21.6, 20.3, -0.5; ${ }^{19}$ F NMR (376 $\left.\mathbf{M H z}, \mathbf{C D C l}_{3}\right)$ : $\delta$-111.5; IR (neat, $\left.\mathbf{c m}^{-1}\right): v 2960,2178,1728,1708,1645,1437,1345,1159,1062,840,757,659$; HRMS calculated for $\mathrm{C}_{24} \mathrm{H}_{32} \mathrm{FN}_{2} \mathrm{O}_{4} \mathrm{SSi}\left(\mathrm{M}+\mathrm{NH}_{4}\right)^{+}$: 491.1831; Found: 491.1818.<smiles>COC(=O)/C=C(\C)c1cc(Cl)ccc1N([13CH3])CC#CC(C)(C)C</smiles>

White solid; m.p.: 76-77 ${ }^{\circ} \mathrm{C}$; $\left(558 \mathrm{mg} ; 57 \%\right.$ yield for two steps); ${ }^{1} \mathbf{H}$ NMR (400 MHz, $\left.\mathbf{C D C l}_{3}\right): \delta 7.62(\mathrm{~d}, J=8.4 \mathrm{~Hz}, 2 \mathrm{H}), 7.26(\mathrm{~d}, J=8.0 \mathrm{~Hz}, 2 \mathrm{H}), 7.21(\mathrm{~s}, 1 \mathrm{H}), 7.15(\mathrm{dd}, J$ $=8.8 \mathrm{~Hz}, 2.8 \mathrm{~Hz}, 1 \mathrm{H}), 6.86(\mathrm{~d}, J=8.8 \mathrm{~Hz}, 1 \mathrm{H}), 5.89(\mathrm{q}, J=1.2 \mathrm{~Hz}, 1 \mathrm{H}), 4.47(\mathrm{br}, 1 \mathrm{H})$, $4.07(\mathrm{br}, 1 \mathrm{H}), 3.71(\mathrm{~s}, 3 \mathrm{H}), 2.52(\mathrm{~d}, J=1.2 \mathrm{~Hz}, 3 \mathrm{H}), 2.41(\mathrm{~s}, 3 \mathrm{H}),-0.05(\mathrm{~s}, 9 \mathrm{H}) ;{ }^{13} \mathrm{C}$ NMR (100 MHz, $\left.\mathbf{C D C l}_{3}\right): \delta$ 166.5, 154.2, 146.8, 144.1, 135.7, 134.6, 130.3, 129.5, 129.2, 128.5, 128.1, 120.7, 98.7, 92.1, 51.2, 42.3, 21.6, 20.3, -0.5; IR (neat, $\mathbf{c m}^{-1}$ ): $v$ 2952, 2190, 1713, 1643, 1342, 1163, 1063, 842, 759, 663; HRMS calculated for $\mathrm{C}_{24} \mathrm{H}_{32} \mathrm{ClN}_{2} \mathrm{O}_{4} \mathrm{SSi}\left(\mathrm{M}+\mathrm{NH}_{4}\right)^{+}:$507.1535; Found: 507.1521. 


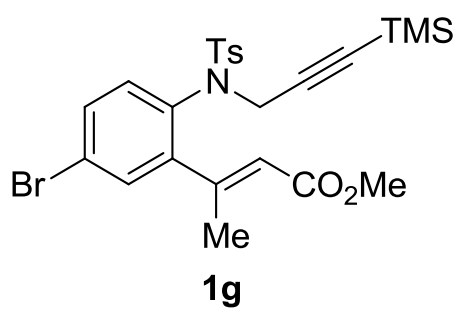

Pale yellow solid; m.p.: $94-95{ }^{\circ} \mathrm{C}$; $\left(630 \mathrm{mg} ; 59 \%\right.$ yield for two steps); ${ }^{\mathbf{1}} \mathbf{H}$ NMR (400 MHz, $\left.\mathbf{C D C l}_{3}\right): \delta 7.63(\mathrm{~d}, J=8.4 \mathrm{~Hz}, 2 \mathrm{H}), 7.38(\mathrm{~d}, J=2.8 \mathrm{~Hz}, 1 \mathrm{H}), 7.28-7.26(\mathrm{~m}, 3 \mathrm{H})$, $6.80(\mathrm{~d}, J=8.4 \mathrm{~Hz}, 1 \mathrm{H}), 5.90(\mathrm{q}, J=1.2 \mathrm{~Hz}, 1 \mathrm{H}), 4.50(\mathrm{br}, 1 \mathrm{H}), 4.10(\mathrm{br}, 1 \mathrm{H}), 3.72(\mathrm{~s}$, 3H), $2.53(\mathrm{~d}, J=0.8 \mathrm{~Hz}, 3 \mathrm{H}), 2.41(\mathrm{~s}, 3 \mathrm{H}),-0.04$ (s, 9H); ${ }^{13} \mathbf{C}$ NMR (100 MHz, $\left.\mathbf{C D C l}_{3}\right): \delta 166.4,154.1,174.0,144.1,135.7,135.1,132.1,131.1,130.6,129.5,128.5$, 122.7, 120.7, 98.7, 92.1, 51.2, 42.2, 21.6, 20.3, -0.5; IR (neat, $\mathbf{~ c m}^{-1}$ ): $v$ 2953, 2177, 1713, 1644, 1345, 1161, 1065, 843, 759, 659, 591; HRMS calculated for $\mathrm{C}_{24} \mathrm{H}_{32} \mathrm{BrN}_{2} \mathrm{O}_{4} \mathrm{SSi}\left(\mathrm{M}+\mathrm{NH}_{4}\right)^{+}:$551.1030; Found: 551.1024 .<smiles>[3H]N(CC#CC(C)(C)C)c1ccc(C(F)(F)F)cc1C(C)=CC(C)=O</smiles>

White solid; m.p.: $112-113{ }^{\circ} \mathrm{C}$; $\left(648 \mathrm{mg} ; 62 \%\right.$ yield for two steps); ${ }^{1} \mathbf{H}$ NMR (400 MHz, $\left.\mathbf{C D C l}_{3}\right): \delta 7.64(\mathrm{~d}, J=8.4 \mathrm{~Hz}, 2 \mathrm{H}), 7.59(\mathrm{~d}, J=8.0 \mathrm{~Hz}, 1 \mathrm{H}), 7.37(\mathrm{~d}, J=8.0$ $\mathrm{Hz}, 1 \mathrm{H}), 7.30(\mathrm{~d}, J=8.0 \mathrm{~Hz}, 2 \mathrm{H}), 7.21(\mathrm{~s}, 1 \mathrm{H}), 5.91(\mathrm{q}, J=1.2 \mathrm{~Hz}, 1 \mathrm{H}), 4.65(\mathrm{br}, 1 \mathrm{H})$, 4.07 (br, 1H), 3.74 (s, 3H), $2.57(\mathrm{~d}, J=1.2 \mathrm{~Hz}, 3 \mathrm{H}), 2.43(\mathrm{~s}, 3 \mathrm{H}),-0.05(\mathrm{~s}, 9 \mathrm{H}) ;{ }^{13} \mathrm{C}$ NMR (100 MHz, $\left.\mathbf{C D C l}_{3}\right): \delta 166.4,154.2,149.0,144.5,136.7,135.6,130.3$ (q, $J=$ $33.0 \mathrm{~Hz}), 129.8,129.6,128.7,126.6(\mathrm{q}, J=3.6 \mathrm{~Hz}), 125.7(\mathrm{~d}, J=3.6 \mathrm{~Hz}), 123.3(\mathrm{q}, J$ $=271.1 \mathrm{~Hz}), 120.9,98.2,92.5,51.3,42.3,21.6,20.3,-0.7 ;{ }^{19}$ F NMR (376 MHz, $\left.\mathbf{C D C l}_{3}\right): \delta-62.9 ;$ IR (neat, $\mathbf{c m}^{-1}$ ): $v 2957,2180,1718,1642,1410,1330,1164,1127$, 1083, 841, 665; HRMS calculated for $\mathrm{C}_{25} \mathrm{H}_{32} \mathrm{~F}_{3} \mathrm{~N}_{2} \mathrm{O}_{4} \mathrm{SSi}\left(\mathrm{M}+\mathrm{NH}_{4}\right)^{+}:$541.1799; Found: 541.1784 . 
<smiles>[3H]N(CC#CC(C)(C)C)c1ccc(-c2ccccc2)cc1C(C)=CCOC</smiles>

$1 \mathrm{i}$

Pale yellow solid; m.p.: 103-104 ${ }^{\circ} \mathrm{C}$; (542 mg; 51\% yield for two steps); ${ }^{1} \mathbf{H}$ NMR (400 MHz, $\left.\mathbf{C D C l}_{3}\right): \delta 7.72(\mathrm{~d}, J=8.4 \mathrm{~Hz}, 2 \mathrm{H}), 7.56(\mathrm{~d}, J=7.6 \mathrm{~Hz}, 2 \mathrm{H}), 7.46-7.41(\mathrm{~m}$, 4H), 7.38-7.35 (m, 1H), $7.30(\mathrm{~d}, J=8.4 \mathrm{~Hz}, 2 \mathrm{H}), 7.04(\mathrm{~d}, J=8.4 \mathrm{~Hz}, 1 \mathrm{H}), 5.99(\mathrm{q}, J=$ $1.6 \mathrm{~Hz}, 1 \mathrm{H}), 4.59$ (br, 1H), 4.18 (br, 1H), 3.76 (s, 3H), 2.62 (d, J = 1.2 Hz, 3H), 2.45 (s, 3H), -0.01 (s, 9H); ${ }^{13} \mathbf{C}$ NMR (100 MHz, $\left.\mathbf{C D C l}_{3}\right): \delta$ 166.9, 156.0, 145.5, 144.0, $141.8,139.8,136.3,135.1,129.6,129.5,129.0,128.7,128.0,127.9,127.2,126.7$, 120.3, 99.2, 91.9, 51.2, 42.5, 21.7, 20.7, -0.4; IR (neat, $\mathbf{~ c m}^{-1}$ ): v 2956, 2173, 1720, 1638, 1482, 1342, 1158, 1030, 988, 841, 665; HRMS calculated for $\mathrm{C}_{30} \mathrm{H}_{37} \mathrm{~N}_{2} \mathrm{O}_{4} \mathrm{SSi}$ $\left(\mathrm{M}+\mathrm{NH}_{4}\right)^{+}:$549.2238; Found: 549.2233.

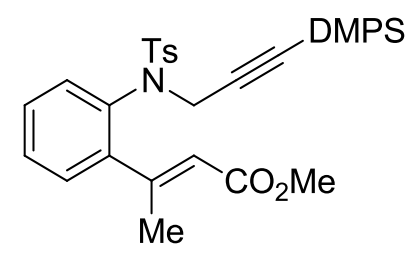

$1 \mathbf{j}$

Colorless oil; (620 mg; 60\% yield for two steps); ${ }^{1} \mathbf{H}$ NMR (400 $\left.\mathbf{M H z}, \mathbf{C D C l}_{3}\right): \delta$ $7.25(\mathrm{~d}, J=8.0 \mathrm{~Hz}, 2 \mathrm{H}), 6.98-6.88(\mathrm{~m}, 6 \mathrm{H}), 6.84-6.79(\mathrm{~m}, 3 \mathrm{H}), 6.72(\mathrm{~d}, J=8.0 \mathrm{~Hz}$, 1H), 7.52 (d, $J=8.0 \mathrm{~Hz}, 1 \mathrm{H}), 5.53(\mathrm{~s}, 1 \mathrm{H}), 4.25$ (br, 1H), 3.78 (br, 1H), 3.30 (s, 3H), 2.18 (s, 3H), 1.98 (s, 3H), -0.17 (s, 6H); ${ }^{13} \mathbf{C}$ NMR (100 MHz, CDCl $): \delta 166.8$, $155.9,145.2$, 143.9, 136.1, 136.0, 135.8, 133.5, 129.6, 129.5, 129.3, 129.0, 128.9, 128.5, 128.2, 127.8, 120.1, 100.8, 89.8, 51.1, 42.4, 21.6, 20.6, -1.3; IR (neat, $\mathbf{c m}^{-1}$ ): $v$ 2953, 2179, 1714, 1639, 1486, 1432, 1348, 1160, 1066, 814, 732, 660; HRMS calculated for $\mathrm{C}_{29} \mathrm{H}_{35} \mathrm{~N}_{2} \mathrm{O}_{4} \mathrm{SSi}\left(\mathrm{M}+\mathrm{NH}_{4}\right)^{+}$: 535.2081; Found: 535.2072. 


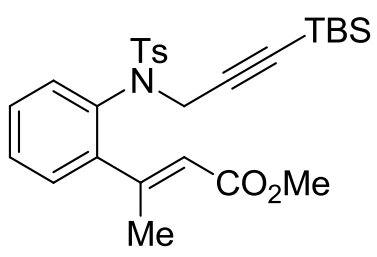

$1 \mathrm{k}$

White solid; m.p.: $72-73{ }^{\circ} \mathrm{C}$; $\left(587 \mathrm{mg}\right.$; $59 \%$ yield for two steps); ${ }^{1} \mathbf{H}$ NMR (400 MHz, $\left.\mathbf{C D C l}_{3}\right): \delta 7.65(\mathrm{~d}, J=8.4 \mathrm{~Hz}, 2 \mathrm{H}), 7.32-7.19(\mathrm{~m}, 5 \mathrm{H}), 6.96(\mathrm{~d}, J=7.6 \mathrm{~Hz}, 1 \mathrm{H}), 5.94$ $(\mathrm{d}, J=1.6 \mathrm{~Hz}, 1 \mathrm{H}), 4.58(\mathrm{br}, 1 \mathrm{H}), 4.21(\mathrm{br}, 1 \mathrm{H}), 3.74(\mathrm{~s}, 3 \mathrm{H}), 2.59$ (d, $J=1.2 \mathrm{~Hz}, 3 \mathrm{H})$, $2.43(\mathrm{~s}, 3 \mathrm{H}), 0.72(\mathrm{~s}, 9 \mathrm{H}),-0.08(\mathrm{~s}, 6 \mathrm{H}) ;{ }^{13} \mathbf{C}$ NMR (100 MHz, CDCl $): \delta 166.9$, $156.0,145.2$, 143.9, 136.0, 135.8, 129.6, 129.3, 129.1, 128.8, 128.5, 128.1, 120.0, 99.5, 90.0, 51.2, 42.3, 25.8, 21.6, 20.5, 16.3, -4.9; IR (neat, $\mathbf{c m}^{-1}$ ): $v$ 2952, 2179, $1706,1632,1348,1185,1162,1070,836,769$; HRMS calculated for $\mathrm{C}_{27} \mathrm{H}_{39} \mathrm{~N}_{2} \mathrm{O}_{4} \mathrm{SSi}$ $\left(\mathrm{M}+\mathrm{NH}_{4}\right)^{+}:$515.2394; Found: 515.2383

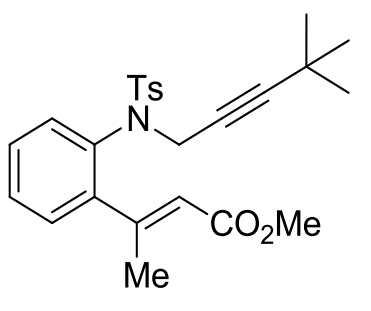

11

Yellow solid; m.p.: $90-92{ }^{\circ} \mathrm{C}$; $\left(553 \mathrm{mg}\right.$; $63 \%$ yield for two steps); ${ }^{1} \mathbf{H}$ NMR (400 MHz, $\left.\mathbf{C D C l}_{3}\right): \delta 7.67(\mathrm{~d}, J=8.4 \mathrm{~Hz}, 2 \mathrm{H}), 7.32-7.18(\mathrm{~m}, 5 \mathrm{H}), 6.95(\mathrm{~d}, J=8.0 \mathrm{~Hz}, 1 \mathrm{H}), 5.94$ $(\mathrm{d}, J=1.6 \mathrm{~Hz}, 1 \mathrm{H}), 4.52$ (br, 1H), 4.13 (br, 1H), 3.75 (s, 3H), $2.58(\mathrm{~d}, J=1.2 \mathrm{~Hz}, 3 \mathrm{H})$, 2.44 (s, 3H), 0.95 (s, 9H); ${ }^{13}$ C NMR (100 MHz, $\left.\mathbf{C D C l}_{3}\right): \delta 166.9,156.1,145.2,143.7$, $136.3,136.0,129.4,129.2,128.7,128.5,128.0,120.0,95.0,72.2,51.1,41.8,30.4$, 27.2, 21.6, 20.6; IR (neat, $\mathbf{c m}^{-1}$ ): v 2926, 2239, 1715, 1639, 1437, 1347, 1160, 1090, 860, 669; HRMS calculated for $\mathrm{C}_{25} \mathrm{H}_{33} \mathrm{~N}_{2} \mathrm{O}_{4} \mathrm{~S}\left(\mathrm{M}+\mathrm{NH}_{4}\right)^{+}$: 457.2156; Found: 457.2140. 


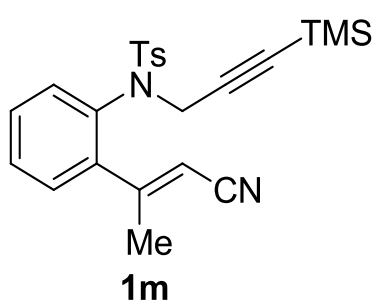

Pale yellow solid; m.p.: $82-83{ }^{\circ} \mathrm{C}$; $\left(590 \mathrm{mg} ; 70 \%\right.$ yield for two steps); ${ }^{\mathbf{1}} \mathbf{H}$ NMR (400 MHz, $\left.\mathbf{C D C l}_{3}\right): \delta 7.60(\mathrm{~d}, J=8.4 \mathrm{~Hz}, 2 \mathrm{H}), 7.37-7.23(\mathrm{~m}, 5 \mathrm{H}), 6.88(\mathrm{~d}, J=8.0 \mathrm{~Hz}, 1 \mathrm{H})$, $5.58(\mathrm{~d}, J=1.2 \mathrm{~Hz}, 1 \mathrm{H}), 4.40(\mathrm{br}, 1 \mathrm{H}), 4.25(\mathrm{br}, 1 \mathrm{H}), 2.50(\mathrm{~d}, J=1.2 \mathrm{~Hz}, 3 \mathrm{H}), 2.45(\mathrm{~s}$, 3H), -0.02 (s, 9H); ${ }^{13} \mathbf{C}$ NMR (100 MHz, $\left.\mathbf{C D C l}_{3}\right): \delta 160.2,144.3,142.0,136.1,135.1$, 129.6, 129.1, 129.0, 128.8, 128.5, 117.0, 100.1, 98.6, 92.2, 42.3, 22.8, 21.6, -0.5; IR $\left(\right.$ neat, $\left.\mathbf{c m}^{-1}\right): v 2957,2214,2190,1621,1341,1159,1072,1007,840,754,659$; HRMS calculated for $\mathrm{C}_{23} \mathrm{H}_{30} \mathrm{~N}_{3} \mathrm{O}_{2} \mathrm{SSi}\left(\mathrm{M}+\mathrm{NH}_{4}\right)^{+}$: 440.1823; Found: 440.1812 .

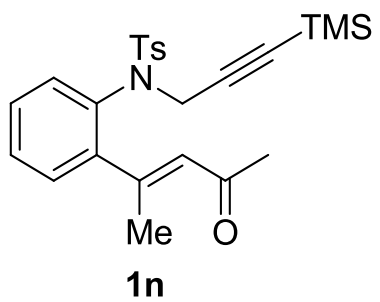

White solid; m.p.: $90-91{ }^{\circ} \mathrm{C}$; (580 mg; 66\% yield for two steps); ${ }^{1} \mathbf{H}$ NMR (400 MHz, $\left.\mathbf{C D C l}_{3}\right): \delta 7.69(\mathrm{~d}, J=8.0 \mathrm{~Hz}, 2 \mathrm{H}), 7.37-7.26(\mathrm{~m}, 4 \mathrm{H}), 7.21(\mathrm{t}, J=8.0 \mathrm{~Hz}, 1 \mathrm{H}), 6.96$ $(\mathrm{d}, J=8.0 \mathrm{~Hz}, 1 \mathrm{H}), 6.42(\mathrm{~s}, 1 \mathrm{H}), 4.55(\mathrm{br}, 1 \mathrm{H}), 4.11(\mathrm{br}, 1 \mathrm{H}), 2.51(\mathrm{~d}, J=1.2 \mathrm{~Hz}, 3 \mathrm{H})$, 2.45 (s, 3H), 2.28 (s, 3H), -0.03 (s, 9H); ${ }^{13} \mathbf{C}$ NMR (100 MHz, CDCl $): \delta 199.2$, $152.8,145.3,143.9,136.3,136.1,129.5,129.3,129.0,128.8,128.5,128.1,128.0$, 99.0, 91.7, 42.3, 32.3, 21.6, 20.9, -0.5; IR (neat, $\mathbf{c m}^{-1}$ ): $v$ 2961, 2172, 1680, 1622, 1485, 1344, 1157, 1057, 843, 761, 665; HRMS calculated for $\mathrm{C}_{24} \mathrm{H}_{33} \mathrm{~N}_{2} \mathrm{O}_{3} \mathrm{SSi}$ $\left(\mathrm{M}+\mathrm{NH}_{4}\right)^{+}:$457.1976; Found: 457.1962. 


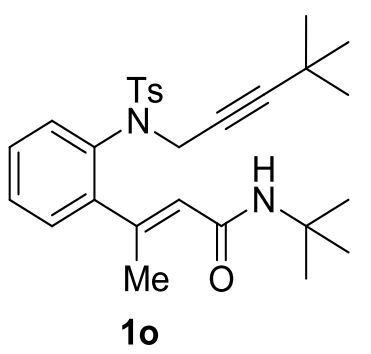

White solid; m.p.: $102-103{ }^{\circ} \mathrm{C}$; (547 mg; $57 \%$ yield for two steps); ${ }^{1} \mathbf{H}$ NMR (400 MHz, $\left.\mathbf{C D C l}_{3}\right): \delta 7.72(\mathrm{~d}, J=8.0 \mathrm{~Hz}, 2 \mathrm{H}), 7.32-7.28(\mathrm{~m}, 4 \mathrm{H}), 7.18(\mathrm{t}, J=8.0 \mathrm{~Hz}, 1 \mathrm{H})$, $6.97(\mathrm{~d}, J=8.0 \mathrm{~Hz}, 1 \mathrm{H}), 5.94(\mathrm{~d}, J=1.2 \mathrm{~Hz}, 1 \mathrm{H}), 5.67(\mathrm{~s}, 1 \mathrm{H}), 4.51(\mathrm{~d}, J=18.0 \mathrm{~Hz}$, 1H), 4.02 (d, $J=18.0 \mathrm{~Hz}, 1 \mathrm{H}), 2.45$ (s, 6H), 1.42 (s, 9H), 0.93 (s, 9H); ${ }^{13} \mathbf{C}$ NMR (100 MHz, $\left.\mathbf{C D C l}_{3}\right): \delta$ 166.7, 146.4, 145.2, 143.7, 136.7, 136.2, 129.5, 129.3, 129.1, 128.6, 128.3, 127.6, 125.3, 94.9, 72.2, 51.2, 41.6, 30.4, 28.9, 27.1, 21.6, 19.7; IR (neat, $\left.\mathbf{c m}^{-1}\right): v 3387,3317,2966,2924,2237,1633,1529,1347,1210,1158,1089,861,761$, 669; HRMS calculated for $\mathrm{C}_{28} \mathrm{H}_{37} \mathrm{~N}_{2} \mathrm{O}_{3} \mathrm{~S}(\mathrm{M}+\mathrm{H})^{+}: 481.2519$; Found: 481.2505 .<smiles>COC(=O)C=C(c1ccccc1)c1ccccc1N(C)C</smiles>

White solid; m.p.: $84-85^{\circ} \mathrm{C}$; (601 mg; $64 \%$ yield for two steps); ${ }^{1} \mathbf{H}$ NMR (400 MHz, $\left.\mathbf{C D C l}_{3}\right): \delta 7.73(\mathrm{~d}, J=8.4 \mathrm{~Hz}, 2 \mathrm{H}), 7.33-7.29(\mathrm{~m}, 3 \mathrm{H}), 7.22-7.18(\mathrm{~m}, 2 \mathrm{H}), 6.99(\mathrm{~d}, J=$ $7.6 \mathrm{~Hz}, 1 \mathrm{H}), 5.84(\mathrm{~s}, 1 \mathrm{H}), 4.61$ (br, 1H), 4.10 (br, 1H), 3.74 (s, 3H), 3.14 (d, $J=6.4$ Hz, 2H), 2.44 (s, 3H), 1.03 (t, $J=7.6 \mathrm{~Hz}, 3 \mathrm{H}),-0.03$ (s, 9H); ${ }^{13} \mathbf{C}$ NMR (100 MHz, $\left.\mathbf{C D C l}_{3}\right): \delta 166.5,162.4,143.9,143.4,136.5,136.3,130.2,129.5,129.2,128.7,128.6$, 128.1, 119.7, 99.0, 91.6, 51.2, 42.4, 25.9, 21.6, 13.2, -0.5; IR (neat, $\mathbf{~ c m}^{-1}$ ): $v 2958$, 2179, 1716, 1633, 1351, 1160, 1091, 1066, 994, 841, 760, 662; HRMS calculated for $\mathrm{C}_{25} \mathrm{H}_{35} \mathrm{~N}_{2} \mathrm{O}_{4} \mathrm{SSi}\left(\mathrm{M}+\mathrm{NH}_{4}\right)^{+}: 487.2081$; Found: 487.2068. 


\section{General procedure for $\mathrm{Pd}\left(\mathrm{CH}_{3} \mathrm{CN}\right)_{4}\left(\mathrm{BF}_{4}\right)_{2} /(\mathrm{R})-\mathrm{SEGPHOS}$ catalyzed}

cyclization of 1<smiles>[R]C#CCN([3H])c1cc[R1]#cc1C([R4])=C[R]</smiles>

1a-1p
$\mathrm{Pd}\left(\mathrm{CH}_{3} \mathrm{CN}\right)_{4}\left(\mathrm{BF}_{4}\right)_{2}(5 \mathrm{~mol} \%)$ (R)-SEGPHOS (5 mol \%)

$\mathrm{CH}_{3} \mathrm{CN}, \mathrm{EtOH}, 60^{\circ} \mathrm{C}$<smiles>[R]C=C1CN([As])c2cc[R1]([H])cc2[C@@]1([R])C[R]</smiles>

$2 a-2 p$

To a dried Schlenk tube were added $\mathrm{Pd}\left(\mathrm{CH}_{3} \mathrm{CN}\right)_{4}\left(\mathrm{BF}_{4}\right)_{2}(2.2 \mathrm{mg}, 0.005 \mathrm{mmol})$, (R)-SEGPHOS (3.1 mg, $0.005 \mathrm{mmol})$, and $\mathrm{CH}_{3} \mathrm{CN}(0.2 \mathrm{~mL})$. The mixture was stirred at room temperature for $1 \mathrm{~min}$, then substrate 1 ( $0.1 \mathrm{mmol}, 1.0$ equiv.) and EtOH (1 $\mathrm{mL}$ ) were added sequentially. The mixture was stirred at $60{ }^{\circ} \mathrm{C}$ until the substrate was disappeared (monitored by TLC). The solvent was removed under reduced pressure and the residue was purified by flash column chromatography (petroleum ether : ethyl acetate $=10: 1-4: 1)$ to give product 2 .<smiles>COC(=O)C=C1CN([As])c2ccccc2[C@@]1(C)CC(C)(C)C</smiles>

$2 a$

White solid; m.p.: $115-116{ }^{\circ} \mathrm{C}$; $(41 \mathrm{mg}$; $90 \%$ yield; $95 \%$ ee); [The ee value was determined by chiral HPLC using a Chiralpak IC-3 column with hexane : isopropanol $=90: 10$ at a flow rate $0.7 \mathrm{~mL} / \mathrm{min}$ detected at $214 \mathrm{~nm}$ wavelength. Elution time: $\mathrm{t}($ minor $)=12.7 \mathrm{~min} ; \mathrm{t}$ (major $)=14.2 \mathrm{~min} ;[\alpha]_{\mathrm{D}}{ }^{27}=-52.07\left(\mathrm{c}=1.0\right.$, in $\left.\mathrm{CDCl}_{3}\right) .{ }^{1} \mathbf{H}$ NMR (400 MHz, CDCl $): \delta 7.79(\mathrm{~d}, J=8.0 \mathrm{~Hz}, 1 \mathrm{H}), 7.42(\mathrm{~d}, J=8.4 \mathrm{~Hz}, 2 \mathrm{H})$, 7.22-7.14 (m, 3H), 7.10(d, $J=8.0 \mathrm{~Hz}, 2 \mathrm{H}), 5.46(\mathrm{~s}, 1 \mathrm{H}), 4.86(\mathrm{~d}, J=15.2 \mathrm{~Hz}, 1 \mathrm{H})$, $4.27(\mathrm{~d}, J=15.6 \mathrm{~Hz}, 1 \mathrm{H}), 3.45(\mathrm{~s}, 3 \mathrm{H}), 2.78(\mathrm{~d}, J=15.2 \mathrm{~Hz}, 1 \mathrm{H}), 2.50(\mathrm{~d}, J=15.2 \mathrm{~Hz}$, 1H), 2.31 (s, 3H), 0.75 (s, 3H), $0.26(\mathrm{~s}, 9 \mathrm{H}) ;{ }^{13} \mathbf{C}$ NMR (100 MHz, CDCl $): \delta 170.8$, $154.4,143.5,137.6,136.3,135.9,129.1,127.6,126.7,126.2,126.0,125.2,124.9$, 51.2, 50.2, 48.6, 41.8, 29.2, 21.4, 0.1; IR (neat, $\mathbf{~ c m}^{-1}$ ): $v$ 2956, 1737, 1604, 1443, 1342, 1254, 1157, 1075, 851, 756, 657; HRMS calculated for $\mathrm{C}_{24} \mathrm{H}_{35} \mathrm{~N}_{2} \mathrm{O}_{4} \mathrm{SSi}$ $\left(\mathrm{M}+\mathrm{NH}_{4}\right)^{+}:$475.2081; Found: 475.2071. 
<smiles>[CH2-][NH+]1CC(=CC(C)=O)[C@@](C)(CC(C)=O)c2cc(C)ccc21</smiles>

2b

White solid; m.p.: $92-93{ }^{\circ} \mathrm{C}$; $(45 \mathrm{mg}$; 96\% yield; 95\% ee); [The ee value was determined by chiral HPLC using a Chiralpak IC-3 column with hexane : isopropanol $=90: 10$ at a flow rate $0.7 \mathrm{~mL} / \mathrm{min}$ detected at $214 \mathrm{~nm}$ wavelength. Elution time: $\mathrm{t}($ minor $)=11.9 \mathrm{~min} ; \mathrm{t}($ major $)=13.5 \mathrm{~min} ;[\alpha]_{\mathrm{D}}{ }^{21}=-124.91\left(\mathrm{c}=1.0\right.$, in $\left.\left.\mathrm{CDCl}_{3}\right)\right] .{ }^{1} \mathbf{H}$ NMR (400 MHz, $\left.\mathbf{C D C l}_{3}\right): \delta 7.66(\mathrm{~d}, J=8.4 \mathrm{~Hz}, 1 \mathrm{H}), 7.41(\mathrm{~d}, J=8.4 \mathrm{~Hz}, 2 \mathrm{H}), 7.10$ $(\mathrm{d}, J=8.0 \mathrm{~Hz}, 2 \mathrm{H}), 7.01(\mathrm{~d}, J=8.0 \mathrm{~Hz}, 1 \mathrm{H}), 6.93(\mathrm{~s}, 1 \mathrm{H}), 5.43(\mathrm{~d}, J=1.2 \mathrm{~Hz}, 1 \mathrm{H})$, $4.83(\mathrm{~d}, J=15.2 \mathrm{~Hz}, 1 \mathrm{H}), 4.25(\mathrm{dd}, J=15.2 \mathrm{~Hz}, 1.2 \mathrm{~Hz}, 1 \mathrm{H}), 3.46$ (s, 3H), 2.75 (d, $J$ $=14.8 \mathrm{~Hz}, 1 \mathrm{H}), 2.46(\mathrm{~d}, J=15.2 \mathrm{~Hz}, 1 \mathrm{H}), 2.31(\mathrm{~s}, 3 \mathrm{H}), 2.29(\mathrm{~s}, 3 \mathrm{H}), 0.74(\mathrm{~s}, 3 \mathrm{H})$, 0.25 (s, 9H); ${ }^{13} \mathbf{C}$ NMR (100 MHz, $\left.\mathbf{C D C l}_{3}\right): \delta$ 171.0, 154.5, 143.4, 137.4, 136.4, $135.6,133.4,129.2,127.8,127.6,126.8,125.2,124.9,51.3,50.3,48.7,41.8,29.2$, 21.5, 21.3, 0.1; IR (neat, $\mathbf{c m}^{-1}$ ): v 2952, 1729, 1604, 1338, 1155, 1045, 848, 669; HRMS calculated for $\mathrm{C}_{25} \mathrm{H}_{37} \mathrm{~N}_{2} \mathrm{O}_{4} \mathrm{SSi}\left(\mathrm{M}+\mathrm{NH}_{4}\right)^{+}$: 489.2238; Found: 489.2224 .<smiles>[CH2-][NH+]1CC(=CC(=O)OC)[C@](C)(CC(C)(C)C)c2ccc(C)cc21</smiles>

2c

Pale yellow solid; m.p.: $108-109^{\circ} \mathrm{C}$; $(43 \mathrm{mg}$; $92 \%$ yield; $94 \%$ ee); [The ee value was determined by chiral HPLC using a Chiralpak IC-3 column with hexane : isopropanol $=90: 10$ at a flow rate $0.7 \mathrm{~mL} / \mathrm{min}$ detected at $214 \mathrm{~nm}$ wavelength. Elution time: $\mathrm{t}($ minor $)=9.9 \mathrm{~min} ; \mathrm{t}($ major $)=10.6 \mathrm{~min} ;[\alpha]_{\mathrm{D}}{ }^{22}=-86.45\left(\mathrm{c}=1.0\right.$, in $\left.\left.\mathrm{CDCl}_{3}\right)\right] .{ }^{1} \mathbf{H}$ NMR (400 MHz, $\left.\mathbf{C D C l}_{3}\right): \delta 7.61(\mathrm{~d}, J=0.8 \mathrm{~Hz}, 1 \mathrm{H}), 7.42(\mathrm{~d}, J=8.4 \mathrm{~Hz}, 2 \mathrm{H}), 7.11$ $(\mathrm{d}, J=8.0 \mathrm{~Hz}, 2 \mathrm{H}), 7.03(\mathrm{~d}, J=8.0 \mathrm{~Hz}, 1 \mathrm{H}), 6.97(\mathrm{~d}, J=1.2 \mathrm{~Hz}, 1 \mathrm{H}), 5.43(\mathrm{~d}, J=1.2$ $\mathrm{Hz}, 1 \mathrm{H}), 4.83(\mathrm{~d}, J=15.2 \mathrm{~Hz}, 1 \mathrm{H}), 4.24(\mathrm{dd}, J=15.6 \mathrm{~Hz}, 1.6 \mathrm{~Hz}, 1 \mathrm{H}), 3.46$ (s, 3H), $2.74(\mathrm{~d}, J=14.8 \mathrm{~Hz}, 1 \mathrm{H}), 2.46$ (d, $J=14.8 \mathrm{~Hz}, 1 \mathrm{H}), 2.34$ (s, 3H), 2.31 (s, 3H), 0.72 (s, 3H), 0.25 (s, 9H); ${ }^{13} \mathbf{C}$ NMR (100 MHz, $\left.\mathbf{C D C l}_{3}\right): \delta 171.0,154.6,143.4,136.5,136.4$, 
$135.8,134.6,129.2,127.8,127.1,126.1,125.7,124.9,51.3,50.4,48.7,41.7,29.3$, 21.5, 21.2, 0.1; IR (neat, $\mathbf{c m}^{-\mathbf{1}}$ ): v 2947, 2913, 1731, 1615, 1499, 1330, 1206, 1154, 1121, 933, 858, 832, 659; HRMS calculated for $\mathrm{C}_{25} \mathrm{H}_{37} \mathrm{~N}_{2} \mathrm{O}_{4} \mathrm{SSi}\left(\mathrm{M}+\mathrm{NH}_{4}\right)^{+}$: 489.2238; Found: 489.2222.<smiles>CC[C@]1(C)/C(=C\C(C)=S)CN([125I])c2ccc(OC)cc21</smiles>

White solid; m.p.: $69-70{ }^{\circ} \mathrm{C}$; $(44 \mathrm{mg} ; 90 \%$ yield; $94 \%$ ee); [The ee value was determined by chiral HPLC using a Chiralpak IC-3 column with hexane : isopropanol $=90: 10$ at a flow rate $0.7 \mathrm{~mL} / \mathrm{min}$ detected at $214 \mathrm{~nm}$ wavelength. Elution time: $\mathrm{t}($ minor $)=16.4 \mathrm{~min} ; \mathrm{t}($ major $)=20.1 \mathrm{~min} ;[\alpha]_{\mathrm{D}}{ }^{21}=-82.33\left(\mathrm{c}=1.0\right.$, in $\left.\left.\mathrm{CDCl}_{3}\right)\right]$.

${ }^{1}$ H NMR (400 MHz, $\left.\mathbf{C D C l}_{3}\right): \delta 7.72(\mathrm{~d}, J=8.8 \mathrm{~Hz}, 1 \mathrm{H}), 7.38(\mathrm{~d}, J=8.4 \mathrm{~Hz}, 2 \mathrm{H})$, $7.09(\mathrm{~d}, J=8.4 \mathrm{~Hz}, 2 \mathrm{H}), 6.78(\mathrm{dd}, J=8.8 \mathrm{~Hz}, 1.2 \mathrm{~Hz}, 1 \mathrm{H}), 6.65(\mathrm{~d}, J=2.4 \mathrm{~Hz}, 1 \mathrm{H})$, $5.42(\mathrm{~d}, J=0.8 \mathrm{~Hz}, 1 \mathrm{H}), 4.86(\mathrm{~d}, J=15.6 \mathrm{~Hz}, 1 \mathrm{H}), 4.22(\mathrm{dd}, J=15.6 \mathrm{~Hz}, 1.6 \mathrm{~Hz}, 1 \mathrm{H})$, $3.78(\mathrm{~s}, 3 \mathrm{H}), 3.46$ (s, 3H), $2.74(\mathrm{~d}, J=15.2 \mathrm{~Hz}, 1 \mathrm{H}), 2.46$ (d, $J=15.2 \mathrm{~Hz}, 1 \mathrm{H}), 2.31$ (s, 3H), 0.65 (s, 3H), 0.25 (s, 9H); ${ }^{13} \mathbf{C}$ NMR (100 MHz, $\left.\mathbf{C D C l}_{\mathbf{3}}\right): \delta 170.9,157.7,154.3$, $143.4,139.3,136.2,129.2,128.9,127.9,127.0,125.2,112.2,111.6,55.4,51.4,50.4$, 49.0, 42.0, 29.4, 21.5, 0.1; IR (neat, $\mathbf{c m}^{-1}$ ): v 2959, 1734, 1603, 1488, 1344, 1289, 1155, 1073, 844, 808, 665; HRMS calculated for $\mathrm{C}_{25} \mathrm{H}_{37} \mathrm{~N}_{2} \mathrm{O}_{5} \mathrm{SSi}\left(\mathrm{M}+\mathrm{NH}_{4}\right)^{+}$: 505.2187; Found: 505.2172.<smiles>COC(=O)C=C1C[NH2+]c2ccc(F)cc2[C@]1(C)CC(C)(C)C</smiles>

2e

White solid; m.p.: $121-122{ }^{\circ} \mathrm{C}$; (39 mg; 82\% yield; 94\% ee); [The ee value was determined by chiral HPLC using a Chiralpak IC column with hexane : isopropanol = 90 : 10 at a flow rate $0.7 \mathrm{~mL} / \mathrm{min}$ detected at $214 \mathrm{~nm}$ wavelength. Elution time: 
$\mathrm{t}($ minor $)=12.7 \mathrm{~min} ; \mathrm{t}($ major $)=14.4 \mathrm{~min} ;[\alpha]_{\mathrm{D}}{ }^{23}=-105.45\left(\mathrm{c}=1.0\right.$, in $\left.\left.\mathrm{CDCl}_{3}\right)\right] .{ }^{1} \mathbf{H}$ NMR (400 MHz, CDCl $): \delta 7.79(\mathrm{dd}, J=8.8 \mathrm{~Hz}, 5.6 \mathrm{~Hz}, 1 \mathrm{H}), 7.39(\mathrm{~d}, J=8.4 \mathrm{~Hz}$, 2H), $7.12(\mathrm{~d}, J=8.0 \mathrm{~Hz}, 2 \mathrm{H}), 6.96-6.91(\mathrm{~m}, 1 \mathrm{H}), 6.84(\mathrm{dd}, J=10 \mathrm{~Hz}, 2.8 \mathrm{~Hz}, 1 \mathrm{H})$, $5.45(\mathrm{~d}, J=1.6 \mathrm{~Hz}, 1 \mathrm{H}), 4.87(\mathrm{~d}, J=15.6 \mathrm{~Hz}, 1 \mathrm{H}), 4.23(\mathrm{dd}, J=15.6 \mathrm{~Hz}, 1.6 \mathrm{~Hz}, 1 \mathrm{H})$, $3.47(\mathrm{~s}, 3 \mathrm{H}), 2.73(\mathrm{~d}, J=15.6 \mathrm{~Hz}, 1 \mathrm{H}), 2.49(\mathrm{~d}, J=15.6 \mathrm{~Hz}, 1 \mathrm{H}), 2.32(\mathrm{~s}, 3 \mathrm{H}), 0.64(\mathrm{~s}$, 3H), 0.26 (s, 9H); ${ }^{13} \mathbf{C}$ NMR (100 MHz, $\left.\mathbf{C D C l}_{3}\right): \delta 170.7,160.8(\mathrm{~d}, J=243.7 \mathrm{~Hz})$, $153.8,143.7,140.2$ (d, $J=6.8 \mathrm{~Hz}), 136.1,132.0(\mathrm{~d}, J=2.3 \mathrm{~Hz}), 129.3,127.8,127.5$ $(\mathrm{d}, J=8.3 \mathrm{~Hz}), 125.6,114.0(\mathrm{~d}, J=22.8 \mathrm{~Hz}), 113.0(\mathrm{~d}, J=23.5 \mathrm{~Hz}), 51.5,50.4,48.9$, 42.0, 29.6, 21.5, 0.1; ${ }^{19}$ F NMR (376 $\left.\mathbf{M H z}, \mathbf{C D C l}_{3}\right): \delta-115.2 ;$ IR (neat, $\left.\mathbf{~ c m}^{-1}\right): v 2953$, 2898, 1742, 1611, 1487, 1345, 1251, 1198, 1156, 1068, 848, 665; HRMS calculated for $\mathrm{C}_{24} \mathrm{H}_{34} \mathrm{FN}_{2} \mathrm{O}_{4} \mathrm{SSi}\left(\mathrm{M}+\mathrm{NH}_{4}\right)^{+}$: 493.1987; Found: 493.1975.

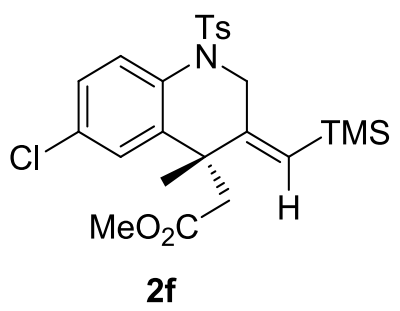

White solid; m.p.: $102-103{ }^{\circ} \mathrm{C}$; $(39 \mathrm{mg} ; 79 \%$ yield; $92 \%$ ee); [The ee value was determined by chiral HPLC using a Chiralpak IC column with hexane $:$ isopropanol = 90 : 10 at a flow rate $0.7 \mathrm{~mL} / \mathrm{min}$ detected at $214 \mathrm{~nm}$ wavelength. Elution time: $\mathrm{t}($ minor $)=11.4 \mathrm{~min} ; \mathrm{t}$ (major $)=12.8 \mathrm{~min} ;[\alpha]_{\mathrm{D}}{ }^{23}=-88.71\left(\mathrm{c}=1.0\right.$, in $\left.\left.\mathrm{CDCl}_{3}\right)\right] .{ }^{1} \mathbf{H}$ NMR (400 MHz, CDCl 3$): \delta 7.76(\mathrm{~d}, J=8.8 \mathrm{~Hz}, 1 \mathrm{H}), 7.43(\mathrm{~d}, J=8.4 \mathrm{~Hz}, 2 \mathrm{H})$, 7.20-7.10 (m, 4H), $5.46(\mathrm{~d}, J=1.2 \mathrm{~Hz}, 1 \mathrm{H}), 4.84(\mathrm{~d}, J=15.6 \mathrm{~Hz}, 1 \mathrm{H}), 4.25(\mathrm{dd}, J=$ $15.6 \mathrm{~Hz}, 1.6 \mathrm{~Hz}, 1 \mathrm{H}), 3.48$ (s, 3H), 2.73 (d, $J=15.6 \mathrm{~Hz}, 1 \mathrm{H}), 2.48$ (d, $J=15.6 \mathrm{~Hz}$, 1H), 2.33 (s, 3H), 0.71 (s, 3H), 0.25 (s, 9H); ${ }^{13} \mathbf{C}$ NMR (100 MHz, CDCl 3 ): $\delta$ 170.7, $153.7,143.8,139.6,136.1,134.7,131.5,129.4,127.8,127.0,126.7,126.4,125.6$, 51.5, 50.2, 48.5, 42.0, 29.5, 21.5, 0.1; IR (neat, $\mathbf{c m}^{-1}$ ): $v 2952,1731,1597,1473$, 1348, 1250, 1160, 1046, 808, 665; HRMS calculated for $\mathrm{C}_{24} \mathrm{H}_{34} \mathrm{ClN}_{2} \mathrm{O}_{4} \mathrm{SSi}$ $\left(\mathrm{M}+\mathrm{NH}_{4}\right)^{+}:$509.1692; Found: 509.1678. 


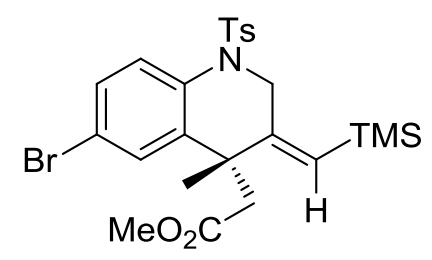

2g

White solid; m.p.: $105-106{ }^{\circ} \mathrm{C}$; $(32 \mathrm{mg} ; 60 \%$ yield; 92\% ee); [The ee value was determined by chiral HPLC using a Chiralpak IG column with hexane : isopropanol = 90 : 10 at a flow rate $0.7 \mathrm{~mL} / \mathrm{min}$ detected at $214 \mathrm{~nm}$ wavelength. Elution time: $\mathrm{t}($ minor $)=13.3 \mathrm{~min} ; \mathrm{t}($ major $)=15.9 \mathrm{~min} ;[\alpha]_{\mathrm{D}}{ }^{28}=-105.32\left(\mathrm{c}=1.0\right.$, in $\left.\left.\mathrm{CDCl}_{3}\right)\right] .{ }^{1} \mathbf{H}$ NMR (400 MHz, CDCl $): \delta 7.69(\mathrm{~d}, J=8.8 \mathrm{~Hz}, 1 \mathrm{H}), 7.44(\mathrm{~d}, J=8.0 \mathrm{~Hz}, 2 \mathrm{H}), 7.33$ (dd, $J=8.8 \mathrm{~Hz}, 2.4 \mathrm{~Hz}, 1 \mathrm{H}), 7.25(\mathrm{~s}, 1 \mathrm{H}), 7.14(\mathrm{~d}, J=8.0 \mathrm{~Hz}, 2 \mathrm{H}), 5.46(\mathrm{~s}, 1 \mathrm{H}), 4.83$ $(\mathrm{d}, J=15.2 \mathrm{~Hz}, 1 \mathrm{H}), 4.26(\mathrm{dd}, J=15.6 \mathrm{~Hz}, 1.6 \mathrm{~Hz}, 1 \mathrm{H}), 3.48(\mathrm{~s}, 3 \mathrm{H}), 2.72(\mathrm{~d}, J=$ $15.6 \mathrm{~Hz}, 1 \mathrm{H}), 2.47(\mathrm{~d}, J=15.6 \mathrm{~Hz}, 1 \mathrm{H}), 2.33(\mathrm{~s}, 3 \mathrm{H}), 0.75(\mathrm{~s}, 3 \mathrm{H}), 0.24(\mathrm{~s}, 9 \mathrm{H}) ;{ }^{13} \mathrm{C}$ NMR (100 MHz, $\left.\mathbf{C D C l}_{3}\right): \delta$ 170.7, 153.7, 143.9, 139.9, 136.1, 135.3, 129.9, 129.4, $129.3,127.7,126.9,125.6,119.4,51.5,50.2,48.4,42.0,29.4,21.5,0.1$; IR (neat, $\left.\mathbf{c m}^{-1}\right): \vee 2945,1735,1599,1466,1340,1172,1071,834,673$; HRMS calculated for $\mathrm{C}_{24} \mathrm{H}_{34} \mathrm{BrN}_{2} \mathrm{O}_{4} \mathrm{SSi}\left(\mathrm{M}+\mathrm{NH}_{4}\right)^{+}:$553.1186; Found: 553.1178 .<smiles>[CH2-][NH+]1CC(=CC(C)=O)[C@@](C)(CC)c2cc(C(F)(F)F)ccc21</smiles>

$2 \mathrm{~h}$

White solid; m.p.: $115-116{ }^{\circ} \mathrm{C}$; $(47 \mathrm{mg}$; $90 \%$ yield; $94 \%$ ee); [The ee value was determined by chiral HPLC using a Chiralpak IC-3 column with hexane : isopropanol $=95: 5$ at a flow rate $0.7 \mathrm{~mL} / \mathrm{min}$ detected at $214 \mathrm{~nm}$ wavelength. Elution time: $\mathrm{t}($ minor $)=6.8 \mathrm{~min} ; \mathrm{t}$ (major $)=7.4 \mathrm{~min} ;[\alpha]_{\mathrm{D}}{ }^{29}=-151.04\left(\mathrm{c}=1.0\right.$, in $\left.\left.\mathrm{CDCl}_{3}\right)\right] .{ }^{1} \mathbf{H}$ NMR (400 MHz, CDCl $\left.\mathbf{3}_{3}\right): \delta 8.08(\mathrm{~d}, J=1.2 \mathrm{~Hz}, 1 \mathrm{H}), 7.48(\mathrm{~d}, J=8.0 \mathrm{~Hz}, 2 \mathrm{H}), 7.38$ $(\mathrm{dd}, J=8.4 \mathrm{~Hz}, 1.6 \mathrm{~Hz}, 1 \mathrm{H}), 7.27(\mathrm{~d}, J=8.8 \mathrm{~Hz}, 1 \mathrm{H}), 7.15(\mathrm{~d}, J=8.0 \mathrm{~Hz}, 2 \mathrm{H}), 5.51(\mathrm{~d}$, $J=1.2 \mathrm{~Hz}, 1 \mathrm{H}), 4.87(\mathrm{~d}, J=15.6 \mathrm{~Hz}, 1 \mathrm{H}), 4.29(\mathrm{dd}, J=15.2 \mathrm{~Hz}, 1.6 \mathrm{~Hz}, 1 \mathrm{H}), 3.47(\mathrm{~s}$, $3 \mathrm{H}), 2.82(\mathrm{~d}, J=15.6 \mathrm{~Hz}, 1 \mathrm{H}), 2.56(\mathrm{~d}, J=15.2 \mathrm{~Hz}, 1 \mathrm{H}), 2.33(\mathrm{~s}, 3 \mathrm{H}), 0.81(\mathrm{~s}, 3 \mathrm{H})$, 0.25 (s, 9H); ${ }^{13} \mathbf{C}$ NMR (100 MHz, $\left.\mathbf{C D C l}_{3}\right): \delta$ 170.7, 153.9, 144.1, 141.5, 136.8, 
136.1, 129.5, $129.2(\mathrm{q}, J=32.9 \mathrm{~Hz}), 127.8,126.9,125.7,123.8(\mathrm{q}, J=270.4 \mathrm{~Hz})$, $122.4(\mathrm{q}, J=3.8 \mathrm{~Hz}), 122.1(\mathrm{q}, J=3.9 \mathrm{~Hz}), 51.5,50.1,48.2,42.2,29.6,21.5,0.1 ;{ }^{19} \mathbf{F}$ NMR (376 MHz, CDCl 3 ): $\delta$-62.5; IR (neat, $\mathbf{c m}^{-\mathbf{1}}$ ): $v$ 2961, 2920, 1737, 1613, 1417 , 1328, 1259, 1084, 1018, 797, 666; HRMS calculated for $\mathrm{C}_{25} \mathrm{H}_{34} \mathrm{~F}_{3} \mathrm{~N}_{2} \mathrm{O}_{4} \mathrm{SSi}$ $\left(\mathrm{M}+\mathrm{NH}_{4}\right)^{+}:$543.1955; Found: 543.1949.<smiles>CC(=O)C=C1CN([As])c2ccc(-c3ccccc3)cc2[C@@]1(C)CC(C)(C)C</smiles>

2i

Pale yellow solid; m.p.: $103-105^{\circ} \mathrm{C}$; $(40 \mathrm{mg}$; $75 \%$ yield; $92 \%$ ee); [The ee value was determined by chiral HPLC using a Chiralpak IF-3 column with hexane : isopropanol $=90: 10$ at a flow rate $0.7 \mathrm{~mL} / \mathrm{min}$ detected at $214 \mathrm{~nm}$ wavelength. Elution time: $\mathrm{t}($ minor $)=8.6 \mathrm{~min} ; \mathrm{t}($ major $)=9.3 \mathrm{~min} ;[\alpha]_{\mathrm{D}}{ }^{28}=-493\left(\mathrm{c}=1.0\right.$, in $\left.\left.\mathrm{CDCl}_{3}\right)\right] .{ }^{\mathbf{1}} \mathbf{H} \mathbf{~ N M R}$ (400 MHz, $\left.\mathbf{C D C l}_{3}\right): \delta 7.86(\mathrm{~d}, J=8.4 \mathrm{~Hz}, 1 \mathrm{H}), 7.55-7.48(\mathrm{~m}, 4 \mathrm{H}), 7.46-7.41(\mathrm{~m}, 3 \mathrm{H})$, 7.37-7.33 (m, 2H), $7.13(\mathrm{~d}, J=8.4 \mathrm{~Hz}, 2 \mathrm{H}), 5.49$ (s, 1H), 4.87 (d, $J=15.2 \mathrm{~Hz}, 1 \mathrm{H})$, $4.32(\mathrm{dd}, J=15.6 \mathrm{~Hz}, 1.6 \mathrm{~Hz}, 1 \mathrm{H}), 3.47$ (s, 3H), 2.84 (d, $J=14.8 \mathrm{~Hz}, 1 \mathrm{H}), 2.54$ (d, $J$ $=14.8 \mathrm{~Hz}, 1 \mathrm{H}), 2.32(\mathrm{~s}, 3 \mathrm{H}), 0.86(\mathrm{~s}, 3 \mathrm{H}), 0.26$ (s, 9H); ${ }^{13} \mathbf{C}$ NMR (100 MHz, CDCl $)$ : $\delta 170.9,154.4,143.6,140.6,138.7,137.8,136.4,135.4,129.3,128.9,127.8,127.5$, $127.0,125.6,125.5,125.2,125.1,51.4,50.4,48.7,42.2,29.3,21.5,0.2$; IR (neat, $\left.\mathbf{c m}^{-1}\right): v 2949,1730,1603,1467,1339,1222,1154,1070,838,755,679 ;$ HRMS calculated for $\mathrm{C}_{30} \mathrm{H}_{39} \mathrm{~N}_{2} \mathrm{O}_{4} \mathrm{SSi}\left(\mathrm{M}+\mathrm{NH}_{4}\right)^{+}$: 551.2394; Found: 551.2383.<smiles>CC(=O)C[C@@]1(C)C(=COC(C)=O)C[NH2+]c2ccccc21</smiles>

2j

Yellow oil; m.p.: $122-123{ }^{\circ} \mathrm{C}$; (44 mg; $85 \%$ yield; $96 \%$ ee); [The ee value was determined by chiral HPLC using a Chiralpak IC-3 column with hexane : isopropanol $=90: 10$ at a flow rate $0.7 \mathrm{~mL} / \mathrm{min}$ detected at $214 \mathrm{~nm}$ wavelength. Elution time: 
$\mathrm{t}($ minor $)=12.7 \mathrm{~min} ; \mathrm{t}($ major $)=14.3 \mathrm{~min} ;[\alpha]_{\mathrm{D}}{ }^{20}=-137.58\left(\mathrm{c}=1.0\right.$, in $\left.\left.\mathrm{CDCl}_{3}\right)\right] .{ }^{1} \mathbf{H}$ NMR (400 MHz, CDCl 3$): \delta 7.72(\mathrm{dd}, J=8.0 \mathrm{~Hz}, 0.8 \mathrm{~Hz}, 1 \mathrm{H}), 7.57-7.55$ (m, 2H), 7.39-7.37 (m, 3H), $7.30(\mathrm{~d}, J=8.4 \mathrm{~Hz}, 2 \mathrm{H}), 7.19-7.15(\mathrm{~m}, 3 \mathrm{H}), 7.01(\mathrm{~d}, J=8.4 \mathrm{~Hz}$, 2H), $5.62(\mathrm{~d}, J=1.2 \mathrm{~Hz}, 1 \mathrm{H}), 4.74(\mathrm{~d}, J=15.2 \mathrm{~Hz}, 1 \mathrm{H}), 4.17(\mathrm{dd}, J=15.6 \mathrm{~Hz}, 1.6 \mathrm{~Hz}$, 1H), 3.43 (s, 3H), 2.83 (d, J = 15.2 Hz, 1H), 2.56 (d, $J=15.2 \mathrm{~Hz}, 1 \mathrm{H}), 2.29$ (s, 3H), 0.79 (s, 3H), 0.54 (s, 6H); ${ }^{13}$ C NMR (100 MHz, $\left.\mathbf{C D C l}_{3}\right): \delta 170.9,156.3,143.4,138.8$, 137.4 , 136.4, 136.1, 133.8, 129.3, 129.2, 128.0, 127.6, 126.8, 126.1, 126.0, 125.1, 122.8, 51.4, 50.6, 48.4, 42.1, 29.5, 21.5, -1.0, -1.0; IR (neat, $\mathbf{c m}^{-1}$ ): v 2953, 2900, 1734, 1601, 1430, 1348, 1159, 1084, 843, 808, 731, 664; HRMS calculated for $\mathrm{C}_{29} \mathrm{H}_{37} \mathrm{~N}_{2} \mathrm{O}_{4} \mathrm{SSi}\left(\mathrm{M}+\mathrm{NH}_{4}\right)^{+}$: 537.2238; Found: 537.2224.

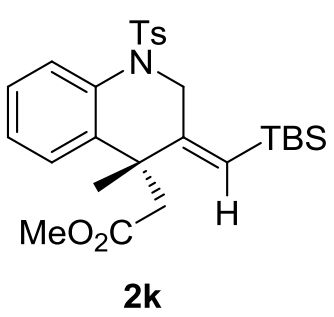

Pale yellow solid; m.p.: $109-111^{\circ} \mathrm{C}$; $(44 \mathrm{mg}$; $89 \%$ yield; $83 \%$ ee); [The ee value was determined by chiral HPLC using a Chiralpak IC-3 column with hexane : isopropanol $=90: 10$ at a flow rate $0.7 \mathrm{~mL} / \mathrm{min}$ detected at $214 \mathrm{~nm}$ wavelength. Elution time: $\mathrm{t}($ minor $)=9.8 \mathrm{~min} ; \mathrm{t}$ (major $)=10.6 \mathrm{~min} ;[\alpha]_{\mathrm{D}}{ }^{26}=-164.98\left(\mathrm{c}=1.0\right.$, in $\left.\left.\mathrm{CDCl}_{3}\right)\right] .{ }^{1} \mathbf{H}$ NMR (400 MHz, CDCl $): \delta 7.77(\mathrm{~d}, J=8.4 \mathrm{~Hz}, 1 \mathrm{H}), 7.46(\mathrm{~d}, J=8.0 \mathrm{~Hz}, 2 \mathrm{H})$, 7.23-7.19 (m, 1H), 7.16-7.10 (m, 4H), $5.52(\mathrm{~d}, J=1.2 \mathrm{~Hz}, 1 \mathrm{H}), 4.85(\mathrm{~d}, J=15.2 \mathrm{~Hz}$, 1H), $4.30(\mathrm{dd}, J=15.2 \mathrm{~Hz}, 1.6 \mathrm{~Hz}, 1 \mathrm{H}), 3.44(\mathrm{~s}, 3 \mathrm{H}), 2.85$ (d, $J=15.6 \mathrm{~Hz}, 1 \mathrm{H}), 2.50$ $(\mathrm{d}, J=15.6 \mathrm{~Hz}, 1 \mathrm{H}), 2.32(\mathrm{~s}, 3 \mathrm{H}), 0.88(\mathrm{~s}, 9 \mathrm{H}), 0.75(\mathrm{~s}, 3 \mathrm{H}), 0.31(\mathrm{~s}, 3 \mathrm{H}), 0.24(\mathrm{~s}, 3 \mathrm{H})$; ${ }^{13}$ C NMR (100 MHz, $\left.\mathbf{C D C l}_{3}\right): \delta 171.0,156.2,143.6,137.6,136.6,136.2,129.3$, 127.7, 126.8, 126.1, 126.0, 125.2, 122.2, 51.4, 50.4, 48.5, 42.1, 30.2, 26.4, 21.5, 17.4, -4.3; IR (neat, $\mathbf{c m}^{-1}$ ): v 2950, 2928, 2854, $1740,1612,1343,1156,1079,1014,849$, 803, 667; HRMS calculated for $\mathrm{C}_{27} \mathrm{H}_{41} \mathrm{~N}_{2} \mathrm{O}_{4} \mathrm{SSi}\left(\mathrm{M}+\mathrm{NH}_{4}\right)^{+}$: 517.2551; Found: 517.2535 . 


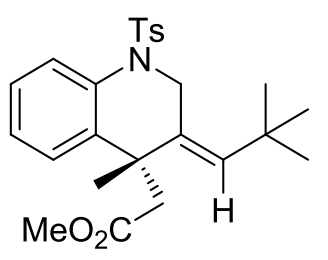

$2 \mathbf{1}$

Pale yellow solid; m.p.: $83-84{ }^{\circ} \mathrm{C}$; $(29 \mathrm{mg} ; 66 \%$ yield; $89 \%$ ee); [The ee value was determined by chiral HPLC using a Chiralpak IC-3 column with hexane : isopropanol $=90: 10$ at a flow rate $0.7 \mathrm{~mL} / \mathrm{min}$ detected at $214 \mathrm{~nm}$ wavelength. Elution time: $\mathrm{t}($ minor $)=15.8 \mathrm{~min} ; \mathrm{t}($ major $)=17.6 \mathrm{~min} ;[\alpha]_{\mathrm{D}}{ }^{27}=-72.92\left(\mathrm{c}=1.0\right.$, in $\left.\left.\mathrm{CDCl}_{3}\right)\right] .{ }^{1} \mathbf{H}$ NMR (400 MHz, CDCl $): \delta 7.77(\mathrm{dd}, J=8.0 \mathrm{~Hz}, 0.8 \mathrm{~Hz}, 1 \mathrm{H}), 7.49(\mathrm{~d}, J=8.4 \mathrm{~Hz}$, 2H), 7.20-7.12 (m, 5H), $5.35(\mathrm{~d}, J=0.8 \mathrm{~Hz}, 1 \mathrm{H}), 5.16(\mathrm{~d}, J=15.2 \mathrm{~Hz}, 1 \mathrm{H}), 4.21(\mathrm{dd}$, $J=15.6 \mathrm{~Hz}, 2.0 \mathrm{~Hz}, 1 \mathrm{H}), 3.47(\mathrm{~s}, 3 \mathrm{H}), 2.79(\mathrm{~d}, J=14.8 \mathrm{~Hz}, 1 \mathrm{H}), 2.45(\mathrm{~d}, J=14.8 \mathrm{~Hz}$, 1H), 2.33 (s, 3H), 1.21 (s, 9H), 0.82 (s, 3H); ${ }^{\mathbf{1 3}} \mathbf{C}$ NMR (100 MHz, CDCl $): \delta$ 171.1, $143.5,137.6$, 136.6, 136.5, 136.1, 136.0, 129.3, 127.6, 126.7, 126.1, 125.8, 124.6, 51.3, 48.8, 46.2, 40.4, 32.8, 31.3, 29.7, 21.5; IR (neat, $\mathbf{c m}^{-1}$ ): v 2953, 1737, 1596, 1440, 1341, 1156, 1077, 875, 760, 672; HRMS calculated for $\mathrm{C}_{25} \mathrm{H}_{35} \mathrm{~N}_{2} \mathrm{O}_{4} \mathrm{~S}$ $\left(\mathrm{M}+\mathrm{NH}_{4}\right)^{+}:$459.2312; Found: 459.2298 .<smiles>CC[C@]1(C)/C(=C\C#N)CN([As])c2ccccc21</smiles>

$2 m$

White solid; m.p.: $101-103{ }^{\circ} \mathrm{C}$; $(31 \mathrm{mg} ; 73 \%$ yield; $87 \%$ ee); [The ee value was determined by chiral HPLC using a Chiralpak IC-3 column with hexane : isopropanol $=90: 10$ at a flow rate $0.7 \mathrm{~mL} / \mathrm{min}$ detected at $214 \mathrm{~nm}$ wavelength. Elution time: $\mathrm{t}($ minor $)=18.4 \mathrm{~min} ; \mathrm{t}($ major $)=22.0 \mathrm{~min} ;[\alpha]_{\mathrm{D}}{ }^{26}=-83.87\left(\mathrm{c}=1.0\right.$, in $\left.\left.\mathrm{CDCl}_{3}\right)\right] .{ }^{1} \mathbf{H}$ NMR (400 MHz, CDCl $): \delta 7.80(\mathrm{~d}, J=8.0 \mathrm{~Hz}, 1 \mathrm{H}), 7.39(\mathrm{~d}, J=8.0 \mathrm{~Hz}, 2 \mathrm{H})$, 7.29-7.23 (m, 3H), $7.13(\mathrm{~d}, J=8.0 \mathrm{~Hz}, 2 \mathrm{H}), 5.65(\mathrm{~s}, 1 \mathrm{H}), 4.71(\mathrm{~d}, J=15.6 \mathrm{~Hz}, 1 \mathrm{H})$, $4.33(\mathrm{dd}, J=15.6 \mathrm{~Hz}, 1.2 \mathrm{~Hz}, 1 \mathrm{H}), 2.47$ (d, $J=16.4 \mathrm{~Hz}, 1 \mathrm{H}), 2.33$ (s, 3H), 2.18 (d, $J$ $=16.8 \mathrm{~Hz}, 1 \mathrm{H}), 1.01$ (s, 3H), 0.29 (s, 9H); $\left.{ }^{13} \mathbf{C ~ N M R ~ ( 1 0 0 ~ M H z , ~} \mathbf{C D C l}_{3}\right): \delta$ 151.3, $143.9,136.5,136.0,135.3,129.4,127.9,127.8,127.5,126.7,126.6,125.8,117.4$, 
50.0, 41.6, 33.0, 27.3, 21.5, 0.1; IR (neat, $\mathbf{c m}^{-1}$ ): v 2957, 2898, 1612, 1487, 1336, $1250,1155,1084,1052,843,775,706,666$; HRMS calculated for $\mathrm{C}_{23} \mathrm{H}_{32} \mathrm{~N}_{3} \mathrm{O}_{2} \mathrm{SSi}$ $\left(\mathrm{M}+\mathrm{NH}_{4}\right)^{+}:$442.1979; Found: 442.1968.

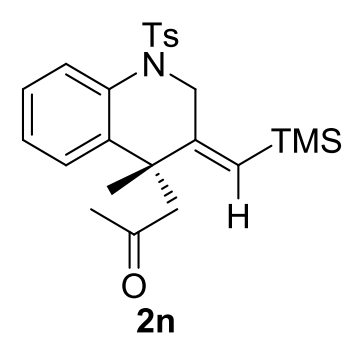

White solid; m.p.: $119-120{ }^{\circ} \mathrm{C}$; (35 mg; $80 \%$ yield; $95 \%$ ee); [The ee value was determined by chiral HPLC using a Chiralpak IC-3 column with hexane : isopropanol $=90: 10$ at a flow rate $0.7 \mathrm{~mL} / \mathrm{min}$ detected at $214 \mathrm{~nm}$ wavelength. Elution time: $\mathrm{t}($ minor $)=15.8 \mathrm{~min} ; \mathrm{t}($ major $)=22.5 \mathrm{~min} ;[\alpha]_{\mathrm{D}}{ }^{28}=-133.54\left(\mathrm{c}=1.0\right.$, in $\left.\left.\mathrm{CDCl}_{3}\right)\right] .{ }^{1} \mathbf{H}$ NMR (400 MHz, CDCl $): \delta 7.75(\mathrm{~d}, J=8.0 \mathrm{~Hz}, 1 \mathrm{H}), 7.47(\mathrm{~d}, J=8.4 \mathrm{~Hz}, 2 \mathrm{H})$, 7.20-7.11 (m, 5H), 5.43 (s, 1H), 4.80 (d, $J=15.2 \mathrm{~Hz}, 1 \mathrm{H}), 4.44$ (dd, $J=15.2 \mathrm{~Hz}, 1.6$ $\mathrm{Hz}, 1 \mathrm{H}), 2.02(\mathrm{~d}, J=16.0 \mathrm{~Hz}, 1 \mathrm{H}), 2.71(\mathrm{~d}, J=16.0 \mathrm{~Hz}, 1 \mathrm{H}), 2.33$ (s, 3H), 1.84 (s, 3H), 0.73 (s, 3H), 0.24 (s, 9H); ${ }^{13} \mathbf{C}$ NMR (100 MHz, $\left.\mathbf{C D C l}_{3}\right): \delta 206.1,155.9,143.5$, 138.0, 136.7, 136.1, 129.4, 127.6, 126.6, 126.0, 125.8, 125.2, 124.1, 57.0, 50.4, 41.8, 31.4, 29.9, 21.5, 0.2; IR (neat, $\mathbf{c m}^{-1}$ ): v 2957, 1720, 1608, 1342, 1250, 1156, 1080, 846, 756, 711, 661; HRMS calculated for $\mathrm{C}_{24} \mathrm{H}_{35} \mathrm{~N}_{2} \mathrm{O}_{3} \mathrm{SSi}\left(\mathrm{M}+\mathrm{NH}_{4}\right)^{+}$: 459.2132; Found: 459.2120 .<smiles>CC(C)(C)/C=C1\CN([125I])c2ccccc2[C@]1(C)CC(=O)NC(C)(C)C</smiles>

20

White solid; m.p.: $126-128{ }^{\circ} \mathrm{C}$; $(34 \mathrm{mg} ; 71 \%$ yield; $90 \%$ ee); [The ee value was determined by chiral HPLC using a Chiralpak IC-3 column with hexane : isopropanol $=90: 10$ at a flow rate $0.7 \mathrm{~mL} / \mathrm{min}$ detected at $214 \mathrm{~nm}$ wavelength. Elution time: 
$\mathrm{t}($ minor $)=14.1 \mathrm{~min} ; \mathrm{t}($ major $)=18.1 \mathrm{~min} ;[\alpha]_{\mathrm{D}}{ }^{28}=-10.21\left(\mathrm{c}=1.0\right.$, in $\left.\left.\mathrm{CDCl}_{3}\right)\right] .{ }^{\mathbf{1}} \mathbf{H}$ NMR (400 MHz, CDCl $)$ ): $\delta 7.69(\mathrm{~d}, J=8.4 \mathrm{~Hz}, 2 \mathrm{H}), 7.39(\mathrm{dd}, J=7.6 \mathrm{~Hz}, 1.6 \mathrm{~Hz}, 1 \mathrm{H})$, 7.31-7.29 (m, 3H), 7.19-7.15 (m, 2H), 5.46 (s, 1H), 5.14 (s, 1H), 4.84 (dd, J= 15.6 Hz, $2.0 \mathrm{~Hz}, 1 \mathrm{H}), 4.47$ (dd, $J=15.2 \mathrm{~Hz}, 1.6 \mathrm{~Hz}, 1 \mathrm{H}), 2.47$ (d, $J=13.6 \mathrm{~Hz}, 1 \mathrm{H}), 2.42$ (s, 3H), $2.32(\mathrm{~d}, J=13.2 \mathrm{~Hz}, 1 \mathrm{H}), 1.40(\mathrm{~s}, 3 \mathrm{H}), 1.17(\mathrm{~s}, 9 \mathrm{H}), 1.04(\mathrm{~s}, 9 \mathrm{H}) ;{ }^{13}$ C NMR (100 MHz, $\left.\mathbf{C D C l}_{3}\right): \delta 169.3,143.9,137.9,137.6,137.0,136.8,135.0,129.9,127.1,126.9$, $126.8,125.8,123.1,50.7,50.4,47.0,42.1,32.8,21.1,28.4,25.8,21.7$; IR (neat, $\left.\mathbf{c m}^{-1}\right): v 3397,2962,1667,1528,1451,1334,1155,1080,773,674$; HRMS calculated for $\mathrm{C}_{28} \mathrm{H}_{39} \mathrm{~N}_{2} \mathrm{O}_{3} \mathrm{~S}(\mathrm{M}+\mathrm{H})^{+}$: 483.2676; Found: 483.2661 .

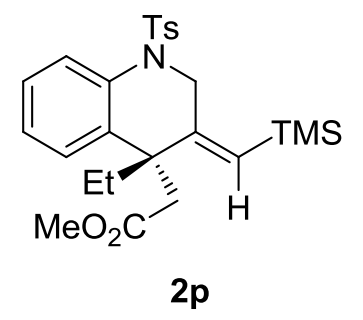

White solid; m.p.: $112-113{ }^{\circ} \mathrm{C}$; $(37 \mathrm{mg} ; 78 \%$ yield; $64 \%$ ee); [The ee value was determined by chiral HPLC using a Chiralpak IC-3 column with hexane : isopropanol $=90: 10$ at a flow rate $0.7 \mathrm{~mL} / \mathrm{min}$ detected at $214 \mathrm{~nm}$ wavelength. Elution time: $\mathrm{t}($ minor $)=10.6 \mathrm{~min} ; \mathrm{t}($ major $)=12.2 \mathrm{~min} ;[\alpha]_{\mathrm{D}}{ }^{27}=-44.78\left(\mathrm{c}=1.0\right.$, in $\left.\left.\mathrm{CDCl}_{3}\right)\right] .{ }^{1} \mathbf{H}$ NMR (400 MHz, CDCl $): \delta 7.68(\mathrm{dd}, J=8.4 \mathrm{~Hz}, 1.2 \mathrm{~Hz}, 1 \mathrm{H}), 7.61(\mathrm{~d}, J=8.4 \mathrm{~Hz}$, 2H), 7.20-7.10 (m, 5H), $5.50(\mathrm{~s}, 1 \mathrm{H}), 4.63(\mathrm{~d}, J=15.2 \mathrm{~Hz}, 1 \mathrm{H}), 4.34(\mathrm{dd}, J=15.2 \mathrm{~Hz}$, $1.6 \mathrm{~Hz}, 1 \mathrm{H}), 3.45$ (s, 3H), 2.80 (d, $J=15.2 \mathrm{~Hz}, 1 \mathrm{H}), 2.35$ (d, $J=15.2 \mathrm{~Hz}, 1 \mathrm{H}), 2.35$ (s, $3 \mathrm{H}), 1.74-1.69(\mathrm{~m}, 1 \mathrm{H}), 1.58-1.53(\mathrm{~m}, 1 \mathrm{H}), 0.34(\mathrm{t}, J=6.8 \mathrm{~Hz}, 3 \mathrm{H}), 0.26(\mathrm{~s}, 9 \mathrm{H}) ;{ }^{13} \mathrm{C}$ NMR (100 MHz, $\left.\mathbf{C D C l}_{3}\right): \delta$ 171.2, 154.0, 143.5, 137.9, 137.7, 134.5, 129.6, 127.2, 126.9, 126.7, 125.1, 124.7, 123.3, 51.3, 20.8, 47.8, 46.5, 35.6, 21.5, 8.6, 0.2; IR (neat, $\left.\mathbf{c m}^{-1}\right): v 2959,1733,1604,1487,1348,1256,1159,1081,1021,838,806,662$; HRMS calculated for $\mathrm{C}_{25} \mathrm{H}_{37} \mathrm{~N}_{2} \mathrm{O}_{4} \mathrm{SSi}(\mathrm{M}+\mathrm{H})^{+}$: 489.2238; Found: 489.2222. 


\section{Procedure for asymmetric cyclization of $1 \mathrm{a}$ in $2 \mathrm{mmoL}$ scale}

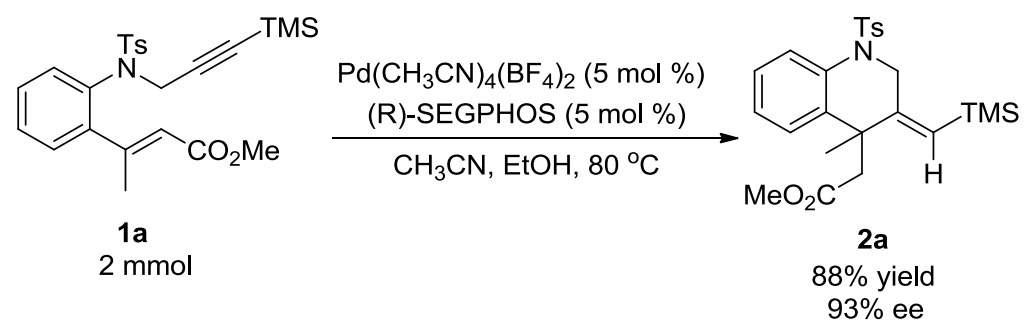

To a dried Schlenk tube were added $\mathrm{Pd}\left(\mathrm{CH}_{3} \mathrm{CN}\right)_{4}\left(\mathrm{BF}_{4}\right)_{2}(44 \mathrm{mg}, 0.1 \mathrm{mmol})$, (R)-SEGPHOS (62 mg, $0.1 \mathrm{mmol}$ ), and $\mathrm{CH}_{3} \mathrm{CN}(4 \mathrm{~mL})$. The mixture was stirred at room temperature for $5 \mathrm{~min}$, then substrate $1 \mathrm{a}(2 \mathrm{mmol}, 1.0$ equiv. $)$ and $\mathrm{EtOH}(20 \mathrm{~mL})$ were added sequentially. The mixture was stirred at $80{ }^{\circ} \mathrm{C}$ overnight. The solvent was removed under reduced pressure and the residue was purified by flash column chromatography (petroleum ether : ethyl acetate $=10: 1-4: 1)$ to give product $2 \mathbf{a}(803$ $\mathrm{mg}, 88 \%$ yield, $93 \%$ ee).

\section{General procedure for synthesis of carbonic ester}<smiles>[R]C#CCN([CH2-])c1cc[R1]#cc1C(C)=CCOC</smiles>

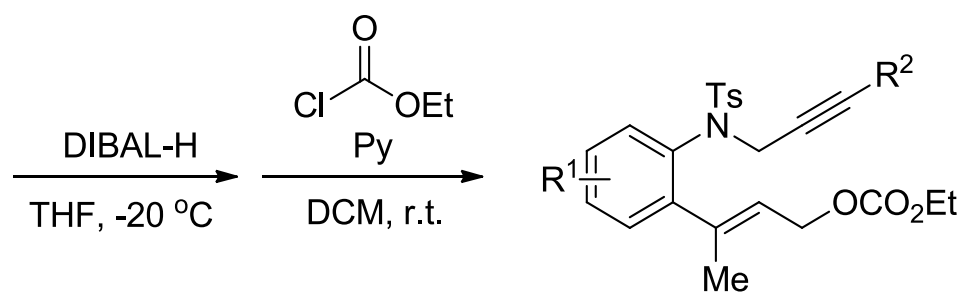

To a stirred solution of carboxylic ester ( $2.0 \mathrm{mmol}, 1.0$ equiv.) in anhydrous THF $(15 \mathrm{~mL})$ was added a solution of DIBAL-H (6 mmol, 3.0 equiv. $1 \mathrm{M}$ in hexane).The resulting solution was stirred at $-20{ }^{\circ} \mathrm{C}$. After completion of the reaction, the crude reaction mixture was quenched by water $(10 \mathrm{~mL})$. The mixture was filtered by celite, washed with ethyl acetate to remove the aluminium salt. The organic layer was dried over anhydrous $\mathrm{Na}_{2} \mathrm{SO}_{4}$, and then evaporated under reduced pressure. The residue was dissolved in DCM (10 mL), and then added pyridine (0.2 mL, 1.2 equiv.) and ethyl chloroformate (325.5 mg, 1.5 equiv.) sequentially. The mixture was stirred at room temperature overnight. After completion of the reaction, the solvent was evaporated under reduced pressure and the residue was purified by flash column chromatography (petroleum ether : ethyl acetate $=5: 1$ ) to get the corresponding carbonic ester. 


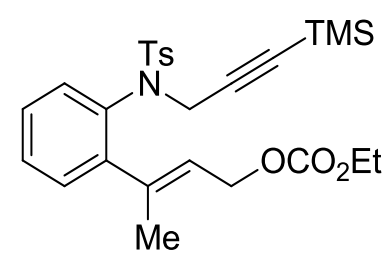

$3 a$

White solid; m.p.: 82-83 ${ }^{\circ} \mathrm{C}$; (719 mg; $72 \%$ yield for two steps); ${ }^{1} \mathbf{H}$ NMR (400 MHz, $\left.\mathbf{C D C l}_{3}\right): \delta 7.73(\mathrm{~d}, J=8.4 \mathrm{~Hz}, 2 \mathrm{H}), 7.31-7.22(\mathrm{~m}, 4 \mathrm{H}), 7.14(\mathrm{td}, J=7.6 \mathrm{~Hz}, 1.6 \mathrm{~Hz}$, 1H), $6.92(\mathrm{~d}, J=7.6 \mathrm{~Hz}, 1 \mathrm{H}), 5.62(\mathrm{td}, J=6.8 \mathrm{~Hz}, 1.2 \mathrm{~Hz}, 1 \mathrm{H}), 4.81(\mathrm{~d}, J=6.8 \mathrm{~Hz}$, 2H), 4.63 (br, 1H), 4.22 (q, J= 7.2 Hz, 2H), 4.08 (br, 1H), 2.45 (s, 3H), 2.17 (s, 3H), $1.32(\mathrm{t}, J=7.2 \mathrm{~Hz}, 3 \mathrm{H}),-0.03(\mathrm{~s}, 9 \mathrm{H}) ;{ }^{13} \mathbf{C} \mathbf{N M R}\left(\mathbf{1 0 0} \mathbf{~ M H z}, \mathbf{C D C l}_{\mathbf{3}}\right): \delta 155.3,145.5$, 143.7, 140.8, 137.1, 136.4, 130.0, 129.4, 129.0, 128.8, 128.6, 127.4, 123.9, 99.5, 91.3, 64.4, 64.1, 42.4, 21.6, 18.4, 14.4, -0.4; IR (neat, $\mathbf{~ c m}^{-1}$ ): v 2961, 2178, 1741, 1596, 1350, 1248, 1159, 1085, 997, 843, 755, 659; HRMS calculated for $\mathrm{C}_{26} \mathrm{H}_{37} \mathrm{~N}_{2} \mathrm{O}_{5} \mathrm{SSi}$ $\left(\mathrm{M}+\mathrm{NH}_{4}\right)^{+}:$517.2187; Found: 517.5178.

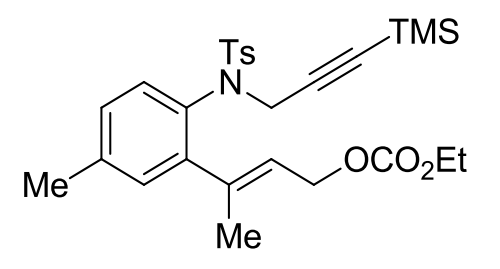

3b

White solid; m.p.: 83-84 ${ }^{\circ} \mathrm{C}$; (698 mg; $68 \%$ yield for two steps); ${ }^{1} \mathbf{H}$ NMR (400 MHz, $\left.\mathbf{C D C l}_{3}\right): \delta 7.72(\mathrm{~d}, J=8.4 \mathrm{~Hz}, 2 \mathrm{H}), 7.28(\mathrm{~d}, J=8.0 \mathrm{~Hz}, 2 \mathrm{H}), 7.05(\mathrm{~s}, 1 \mathrm{H}), 6.94(\mathrm{~d}, J=$ $8.0 \mathrm{~Hz}, 1 \mathrm{H}), 6.81(\mathrm{~d}, J=8.0 \mathrm{~Hz}, 1 \mathrm{H}), 5.61(\mathrm{td}, J=6.4 \mathrm{~Hz}, 1.2 \mathrm{~Hz}, 1 \mathrm{H}), 4.80(\mathrm{~d}, J=$ $6.8 \mathrm{~Hz}, 2 \mathrm{H}), 4.66$ (br, 1H), 4.21 (q, J=7.2 Hz, 2H), 4.03 (br, 1H), 2.43 (s, 3H), 2.31 $(\mathrm{s}, 3 \mathrm{H}), 2.16(\mathrm{~s}, 3 \mathrm{H}), 1.31(\mathrm{t}, J=7.2 \mathrm{~Hz}, 3 \mathrm{H}),-0.02(\mathrm{~s}, 9 \mathrm{H}) ;{ }^{13} \mathbf{C}$ NMR (100 MHz, $\left.\mathbf{C D C l}_{3}\right): \delta 155.1,145.0,143.4,140.8,138.6,137.0,133.7,130.4,129.3,128.7,128.4$, 128.0, 123.5, 99.5, 91.0, 64.2, 63.9, 42.3, 21.5, 21.0, 18.3, 14.3, -0.5; IR (neat, $\mathbf{~ c m}^{-1}$ ): $v$ 2960, 2178, 1741, 1349, 1248, 1158, 1085, 997, 842, 661; HRMS calculated for $\mathrm{C}_{27} \mathrm{H}_{39} \mathrm{~N}_{2} \mathrm{O}_{5} \mathrm{SSi}\left(\mathrm{M}+\mathrm{NH}_{4}\right)^{+}:$531.2343; Found: 531.2333. 


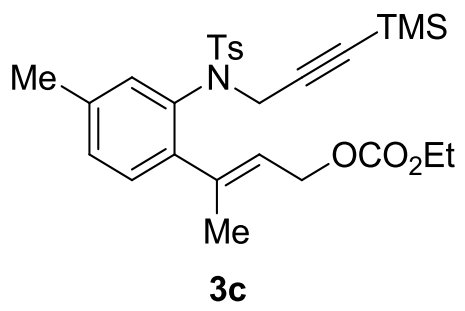

White solid; m.p.: $79-80{ }^{\circ} \mathrm{C}$; $\left(688 \mathrm{mg} ; 67 \%\right.$ yield for two steps); ${ }^{1} \mathbf{H}$ NMR (400 MHz, $\left.\mathbf{C D C l}_{3}\right): \delta 7.74(\mathrm{~d}, J=8.4 \mathrm{~Hz}, 2 \mathrm{H}), 7.30(\mathrm{~d}, J=8.0 \mathrm{~Hz}, 2 \mathrm{H}), 7.13-7.09(\mathrm{~m}, 2 \mathrm{H}), 6.75$ (s, 1H), 5.59 (td, $J=6.8 \mathrm{~Hz}, 1.6 \mathrm{~Hz}, 1 \mathrm{H}), 4.79$ (d, $J=6.8 \mathrm{~Hz}, 2 \mathrm{H}), 4.66$ (br, 1H), 4.22 (q, $J=7.2 \mathrm{~Hz}, 2 \mathrm{H}), 4.03(\mathrm{br}, 1 \mathrm{H}), 2.45(\mathrm{~s}, 3 \mathrm{H}), 2.20(\mathrm{~s}, 3 \mathrm{H}), 2.15(\mathrm{~s}, 3 \mathrm{H}), 1.32(\mathrm{t}, J=$ $7.2 \mathrm{~Hz}, 3 \mathrm{H}),-0.02(\mathrm{~s}, 9 \mathrm{H}) ;{ }^{13} \mathbf{C}$ NMR (100 MHz, $\left.\mathbf{C D C l}_{\mathbf{3}}\right): \delta$ 155.2, 143.6, 142.3, $140.7,137.2,137.1,136.1,129.8,129.7,129.5,129.3,128.6,123.6,99.5,91.1,64.4$, 64.0, 42.3, 21.6, 20.8, 18.4, 14.3, -0.4; IR (neat, $\mathbf{~ c m}^{-1}$ ): $v$ 2960, 2178, 1741, 1350, 1249, 1160, 1085, 996, 841, 660; HRMS calculated for $\mathrm{C}_{27} \mathrm{H}_{39} \mathrm{~N}_{2} \mathrm{O}_{5} \mathrm{SSi}\left(\mathrm{M}+\mathrm{NH}_{4}\right)^{+}$: 531.2343; Found: 531.2331.<smiles>[3H]N(CC#CC(C)(C)C)c1ccc(OC)cc1C(C)=CCOCC</smiles>

White solid; m.p.: $86-87^{\circ} \mathrm{C}$; $\left(656 \mathrm{mg}\right.$; $62 \%$ yield for two steps); ${ }^{1} \mathbf{H}$ NMR (400 MHz, $\left.\mathbf{C D C l}_{3}\right): \delta 7.72(\mathrm{~d}, J=8.4 \mathrm{~Hz}, 2 \mathrm{H}), 7.29(\mathrm{~d}, J=8.0 \mathrm{~Hz}, 2 \mathrm{H}), 6.83(\mathrm{~d}, J=8.8 \mathrm{~Hz}, 1 \mathrm{H})$, $6.75(\mathrm{~d}, J=2.8 \mathrm{~Hz}, 1 \mathrm{H}), 6.65(\mathrm{dd}, J=8.8 \mathrm{~Hz}, 2.8 \mathrm{~Hz}, 1 \mathrm{H}), 5.62(\mathrm{t}, J=6.8 \mathrm{~Hz}, 1 \mathrm{H})$, $4.80(\mathrm{~d}, J=6.8 \mathrm{~Hz}, 2 \mathrm{H}), 4.67(\mathrm{~d}, J=17.6 \mathrm{~Hz}, 1 \mathrm{H}), 4.22(\mathrm{q}, J=7.2 \mathrm{~Hz}, 2 \mathrm{H}), 4.10(\mathrm{~d}, J$ $=17.6 \mathrm{~Hz}, 1 \mathrm{H}), 3.78(\mathrm{~s}, 3 \mathrm{H}), 2.43(\mathrm{~s}, 3 \mathrm{H}), 2.16(\mathrm{~s}, 3 \mathrm{H}), 1.32(\mathrm{t}, J=7.2 \mathrm{~Hz}, 3 \mathrm{H}),-0.09$ (s, 9H); ${ }^{13} \mathbf{C}$ NMR (100 MHz, $\left.\mathbf{C D C l}_{3}\right): \delta$ 159.3, 155.2, 146.7, 143.5, 140.6, 137.0, $130.1,129.3,129.0,128.5,123.7,114.7,112.7,99.6,91.1,64.3,64.0,55.5,42.5,21.5$, 18.3, 14.3, -0.5; IR (neat, $\mathbf{c m}^{-1}$ ): $v$ 2959, 2178, 1741, 1599, 1348, 1248, 1158, 1086, 997, 842, 661; HRMS calculated for $\mathrm{C}_{27} \mathrm{H}_{39} \mathrm{~N}_{2} \mathrm{O}_{6} \mathrm{SSi}\left(\mathrm{M}+\mathrm{NH}_{4}\right)^{+}$: 547.2293; Found: 547.2281 


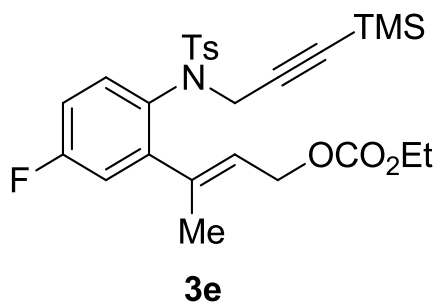

White solid; m.p.: $60-61{ }^{\circ} \mathrm{C}$; $\left(672 \mathrm{mg}\right.$; $65 \%$ yield for two steps); ${ }^{1} \mathbf{H}$ NMR (400 MHz, $\left.\mathbf{C D C l}_{3}\right): \delta 7.70(\mathrm{~d}, J=8.4 \mathrm{~Hz}, 2 \mathrm{H}), 7.30(\mathrm{~d}, J=8.0 \mathrm{~Hz}, 2 \mathrm{H}), 7.30(\mathrm{dd}, J=8.8 \mathrm{~Hz}, 2.8$ $\mathrm{Hz}, 1 \mathrm{H}), 6.90-6.81(\mathrm{~m}, 2 \mathrm{H}), 5.64(\mathrm{td}, J=6.8 \mathrm{~Hz}, 1.2 \mathrm{~Hz}, 1 \mathrm{H}), 4.80(\mathrm{~d}, J=6.8 \mathrm{~Hz}, 2 \mathrm{H})$, 4.66 (br, 1H), 4.22 (q, J = 7.2 Hz, 2H), 4.01 (br, 1H), 2.44 (s, 3H), 2.16 (s, 3H), 1.32 $(\mathrm{t}, J=7.2 \mathrm{~Hz}, 3 \mathrm{H}),-0.02(\mathrm{~s}, 9 \mathrm{H}) ;{ }^{\mathbf{1 3}} \mathbf{C}$ NMR (100 MHz, $\left.\mathbf{C D C l}_{\mathbf{3}}\right): \delta 162.1(\mathrm{~d}, J=248.2$ Hz), 155.2, 147.7 (d, $J=8.4 \mathrm{~Hz}), 143.8,139.5,136.6,132.3$ (d, $J=3.0 \mathrm{~Hz}), 130.7$ (d, $J=9.1 \mathrm{~Hz}), 129.5,128.5,124.6,116.8(\mathrm{~d}, J=22.8 \mathrm{~Hz}), 114.1(\mathrm{~d}, J=22.8 \mathrm{~Hz}), 99.2$, 91.6, 64.2, 64.1, 42.4, 21.6, 18.2, 14.3, -0.5; IR (neat, $\mathbf{c m}^{-1}$ ): $v 2961,2178,1742$, 1589, 1484, 1352, 1249, 1159, 1085, 997, 842, 661; HRMS calculated for $\mathrm{C}_{26} \mathrm{H}_{36} \mathrm{FN}_{2} \mathrm{O}_{5} \mathrm{SSi}\left(\mathrm{M}+\mathrm{NH}_{4}\right)^{+}$: 535.2093; Found: 535.2079.<smiles>[3H]N(CC#CC#N)c1ccc(Cl)cc1/C(C)=C/COC(=O)OCC</smiles>

White solid; m.p.: $82-83{ }^{\circ} \mathrm{C}$; $\left(726 \mathrm{mg}\right.$; $68 \%$ yield for two steps); ${ }^{1} \mathbf{H}$ NMR (400 MHz, $\left.\mathbf{C D C l}_{3}\right): \delta 7.70(\mathrm{~d}, J=8.0 \mathrm{~Hz}, 2 \mathrm{H}), 7.30(\mathrm{~d}, J=8.0 \mathrm{~Hz}, 2 \mathrm{H}), 7.25(\mathrm{~d}, J=2.4 \mathrm{~Hz}, 1 \mathrm{H})$, $7.12(\mathrm{dd}, J=8.4 \mathrm{~Hz}, 2.4 \mathrm{~Hz}, 1 \mathrm{H}), 6.85(\mathrm{~d}, J=8.4 \mathrm{~Hz}, 1 \mathrm{H}), 5.64(\mathrm{td}, J=6.4 \mathrm{~Hz}, 1.6 \mathrm{~Hz}$, $1 \mathrm{H}), 4.80(\mathrm{~d}, J=6.8 \mathrm{~Hz}, 2 \mathrm{H}), 4.60(\mathrm{br}, 1 \mathrm{H}), 4.22(\mathrm{q}, J=7.2 \mathrm{~Hz}, 2 \mathrm{H}), 4.02(\mathrm{br}, 1 \mathrm{H})$, $2.44(\mathrm{~s}, 3 \mathrm{H}), 2.15(\mathrm{~s}, 3 \mathrm{H}), 1.32(\mathrm{t}, J=7.2 \mathrm{~Hz}, 3 \mathrm{H}),-0.02(\mathrm{~s}, 9 \mathrm{H}) ;{ }^{13} \mathbf{C}$ NMR (100 MHz, $\left.\mathbf{C D C l}_{3}\right): \delta 155.1,147.0,143.9,139.3,136.5,135.0,134.4,130.2,129.9,129.5,128.4$, 127.4, 124.8, 99.0, 91.6, 64.0, 42.2, 21.5, 18.1, 14.3, -0.5; IR (neat, $\mathbf{c m}^{-1}$ ): $v$ 2961, 2178, 1742, 1593, 1352, 1249, 1160, 1085, 998, 843, 796, 662; HRMS calculated for $\mathrm{C}_{26} \mathrm{H}_{36} \mathrm{ClN}_{2} \mathrm{O}_{5} \mathrm{SSi}\left(\mathrm{M}+\mathrm{NH}_{4}\right)^{+}:$551.1797; Found: 551.1786. 
<smiles>CCOCC=C(C)c1cc(Br)ccc1N([13CH3])CC#CC(C)(C)C</smiles>

White solid; m.p.: $103-104{ }^{\circ} \mathrm{C}$; (705 mg; 61\% yield for two steps); ${ }^{1} \mathbf{H}$ NMR (400 MHz, $\left.\mathbf{C D C l}_{3}\right): \delta 7.70(\mathrm{~d}, J=8.4 \mathrm{~Hz}, 2 \mathrm{H}), 7.40(\mathrm{~d}, J=2.4 \mathrm{~Hz}, 1 \mathrm{H}), 7.32-7.26(\mathrm{~m}, 3 \mathrm{H})$, $6.78(\mathrm{~d}, J=8.8 \mathrm{~Hz}, 1 \mathrm{H}), 5.63(\mathrm{td}, J=6.8 \mathrm{~Hz}, 1.2 \mathrm{~Hz}, 1 \mathrm{H}), 4.79(\mathrm{~d}, J=6.8 \mathrm{~Hz}, 2 \mathrm{H})$, $4.61(\mathrm{br}, 1 \mathrm{H}), 4.22$ (q, J = 7.2 Hz, 2H), 4.03 (br, 1H), 2.44 (s, 3H), 2.15 (s, 3H), 1.32 $(\mathrm{t}, J=7.2 \mathrm{~Hz}, 3 \mathrm{H}),-0.02(\mathrm{~s}, 9 \mathrm{H}) ;{ }^{13} \mathbf{C}$ NMR (100 MHz, $\left.\mathbf{C D C l}_{3}\right): \delta$ 155.2, 147.4, $143.9,139.3,136.6,135.5,132.9,130.5,130.5,129.5,128.5,124.8,122.7,99.0,91.7$ 64.1, 42.2, 21.6, 18.2, 14.3, -0.5; IR (neat, $\mathbf{~ c m}^{-1}$ ): $v$ 2974, 2177, 1739, 1465, 1352, 1259, 1159, 1083, 989, 645, 786, 663; HRMS calculated for $\mathrm{C}_{26} \mathrm{H}_{36} \mathrm{BrN}_{2} \mathrm{O}_{5} \mathrm{SSi}$ $\left(\mathrm{M}+\mathrm{NH}_{4}\right)^{+}:$595.1292; Found: 595.1284.<smiles>[3H]N(CC#CC#CCCOCC)c1ccc(C(F)(F)F)cc1C(C)=CCOCC</smiles>

$3 h$

White solid; m.p.: 101-102 ${ }^{\circ} \mathrm{C}$; (715 mg; $63 \%$ yield for two steps); ${ }^{1} \mathbf{H}$ NMR (400 MHz, $\left.\mathbf{C D C l}_{3}\right): \delta 7.70(\mathrm{~d}, J=8.4 \mathrm{~Hz}, 2 \mathrm{H}), 7.57(\mathrm{~d}, J=8.0 \mathrm{~Hz}, 1 \mathrm{H}), 7.38(\mathrm{~d}, J=8.4$ $\mathrm{Hz}, 1 \mathrm{H}), 7.33$ (d, $J=8.4 \mathrm{~Hz}, 2 \mathrm{H}), 7.19(\mathrm{~s}, 1 \mathrm{H}), 5.66(\mathrm{t}, J=6.8 \mathrm{~Hz}, 1 \mathrm{H}), 4.83$ (d, $J=$ $6.8 \mathrm{~Hz}, 2 \mathrm{H}), 4.73$ (br, 1H), 4.22 (q, $J=7.2 \mathrm{~Hz}, 2 \mathrm{H}), 4.04$ (br, 1H), 2.45 (s, 3H), 2.19 (s, 3H), 1.32 (t, $J=7.2 \mathrm{~Hz}, 3 \mathrm{H}),-0.04$ (s, 9H); ${ }^{13} \mathbf{C}$ NMR (100 MHz, CDCl $)$ : $\delta$ 155.2, 149.3, 144.2, 139.4, 137.0, 136.3, 130.5, 129.6 (q, $J=32.7 \mathrm{~Hz}), 129.5,128.6,126.5$ $(\mathrm{q}, J=3.7 \mathrm{~Hz}), 125.5(\mathrm{q}, J=3.6 \mathrm{~Hz}), 125.1,123.5(\mathrm{q}, J=270.9 \mathrm{~Hz}), 98.5,92.1,64.1$, 64.1, 42.2, 21.6, 18.2, 14.3, -0.7; IR (neat, $\mathbf{~ c m}^{-1}$ ): $v$ 2963, 2179, 1743, 1409, 1250, 1163, 1125, 1082, 842, 728, 665; HRMS calculated for $\mathrm{C}_{27} \mathrm{H}_{36} \mathrm{~F}_{3} \mathrm{~N}_{2} \mathrm{O}_{5} \mathrm{SSi}\left(\mathrm{M}+\mathrm{NH}_{4}\right)^{+}$: 585.2061; Found: 585.2051. 


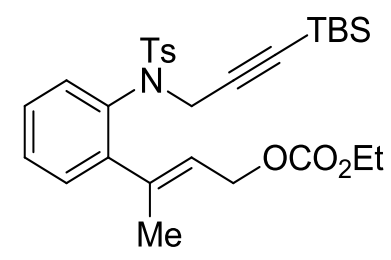

$3 \mathbf{i}$

White solid; m.p.: $83-84{ }^{\circ} \mathrm{C}$; (639 mg; $59 \%$ yield for two steps); ${ }^{1}$ H NMR (400 MHz, $\left.\mathbf{C D C l}_{3}\right): \delta 7.71(\mathrm{~d}, J=8.4 \mathrm{~Hz}, 2 \mathrm{H}), 7.30-7.22(\mathrm{~m}, 4 \mathrm{H}), 7.12(\mathrm{~d}, J=8.0 \mathrm{~Hz}, 1 \mathrm{H}), 6.93$ $(\mathrm{d}, J=8.0 \mathrm{~Hz}, 1 \mathrm{H}), 5.64(\mathrm{td}, J=6.8 \mathrm{~Hz}, 1.2 \mathrm{~Hz}, 1 \mathrm{H}), 4.80(\mathrm{~d}, J=6.8 \mathrm{~Hz}, 2 \mathrm{H}), 4.66$ (br, 1H), 4.22 (q, J=7.2 Hz, 2H), $4.13(\mathrm{br}, 1 \mathrm{H}), 2.43(\mathrm{~s}, 3 \mathrm{H}), 2.18(\mathrm{~s}, 3 \mathrm{H}), 1.32(\mathrm{t}, J=$ $7.2 \mathrm{~Hz}, 3 \mathrm{H}), 0.72$ (s, 9H), -0.09 (s, 6H); ${ }^{13} \mathbf{C}$ NMR (100 MHz, CDCl $\left.\mathbf{3}\right): \delta 155.3,145.3$, 143.6, 140.7, 137.0, 136.2, 129.9, 129.5, 129.1, 128.7, 128.4, 127.4, 123.9, 99.9, 89.6, 64.4, 64.0, 42.2, 25.9, 21.6, 18.4, 16.3, 14.4, -4.8; IR (neat, $\mathbf{~ c m}^{-1}$ ): v 2930, 2856, 2178, 1742, 1351, 1249, 1160, 1085, 999, 816, 771, 661; HRMS calculated for $\mathrm{C}_{29} \mathrm{H}_{43} \mathrm{~N}_{2} \mathrm{O}_{5} \mathrm{SSi}\left(\mathrm{M}+\mathrm{NH}_{4}\right)^{+}:$559.2656; Found: 559.2646.

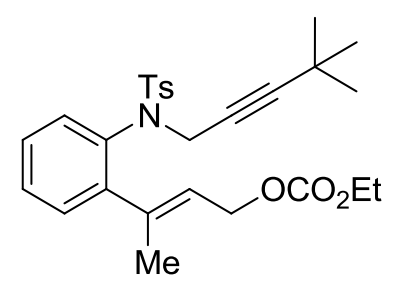

3j

Colorless oil; (590 mg; 61\% yield for two steps); ${ }^{1} \mathbf{H}$ NMR (400 $\left.\mathbf{M H z}, \mathbf{C D C l}_{3}\right): \delta$ $7.72(\mathrm{~d}, J=8.0 \mathrm{~Hz}, 2 \mathrm{H}), 7.31-7.22(\mathrm{~m}, 4 \mathrm{H}), 7.13(\mathrm{~d}, J=7.6 \mathrm{~Hz}, 1 \mathrm{H}), 6.91(\mathrm{~d}, J=8.0$ $\mathrm{Hz}, 1 \mathrm{H}), 5.64(\mathrm{t}, J=6.8 \mathrm{~Hz}, 1 \mathrm{H}), 4.82(\mathrm{~d}, J=6.8 \mathrm{~Hz}, 2 \mathrm{H}), 4.60(\mathrm{br}, 1 \mathrm{H}), 4.22(\mathrm{q}, J=$ $7.2 \mathrm{~Hz}, 2 \mathrm{H}), 4.06$ (br, 1H), 2.43 (s, 3H), 2.18 (s, 3H), 1.32 (t, J= 7.2 Hz, 3H), 0.94 (s, 9H); ${ }^{13} \mathbf{C}$ NMR (100 MHz, $\left.\mathbf{C D C l}_{3}\right): \delta$ 155.2, 145.4, 143.5, 140.8, 137.2, 136.4, 129.8, 129.4, 129.1, 128.6, 128.5, 127.2, 123.8, 94.7, 72.4, 64.4, 64.0, 41.8, 30.4, 27.1, 21.5, 18.3, 14.3; IR (neat, $\mathbf{c m}^{-1}$ ): v 2968, 2240, 1741, 1596, 1446, 1349, 1250, 1159, 1089, 1005, 865, 666; HRMS calculated for $\mathrm{C}_{27} \mathrm{H}_{37} \mathrm{~N}_{2} \mathrm{O}_{5} \mathrm{~S}\left(\mathrm{M}+\mathrm{NH}_{4}\right)^{+}:$501.2418; Found: 501.2404 . 


\section{General procedure for $\operatorname{Pd}\left(\mathrm{CH}_{3} \mathrm{CN}\right)_{4}\left(\mathrm{BF}_{4}\right)_{2} /(\mathrm{R})$-SEGPHOS catalyzed}

cyclization of 3 .<smiles></smiles>

3a-3j
$\mathrm{Pd}\left(\mathrm{CH}_{3} \mathrm{CN}\right)_{4}\left(\mathrm{BF}_{4}\right)_{2}(5 \mathrm{~mol} \%)$ R)-SEGPHOS (5 mol \%)

$\mathrm{CH}_{3} \mathrm{CN}, \mathrm{EtOH}, 80^{\circ} \mathrm{C}$<smiles>[R]/C=C1/CN([As])C2=C(C=C[R1]C=C2)[C@@]1(C)C=C</smiles>

$4 a-4 j$

To a dried Schlenk tube were added $\mathrm{Pd}\left(\mathrm{CH}_{3} \mathrm{CN}\right)_{4}\left(\mathrm{BF}_{4}\right)_{2}(2.2 \mathrm{mg}, 0.005 \mathrm{mmol})$, (R)-SEGPHOS (3.1 mg, $0.005 \mathrm{mmol})$, and $0.2 \mathrm{~mL}$ of $\mathrm{CH}_{3} \mathrm{CN}$. The mixture was stirred at room temperature for $1 \mathrm{~min}$, then substrate 3 ( $0.1 \mathrm{mmol}, 1.0$ equiv.) and 1 $\mathrm{mL}$ of EtOH were added sequentially. The reaction was heated at $80{ }^{\circ} \mathrm{C}$ until the substrate was disappeared (monitored by TLC). The solvents were evaporated under reduced pressure and the residue was purified by flash column chromatography (petroleum ether : ethyl acetate $=20: 1-10: 1$ ) to give product 4 .<smiles>C=C[C@]1(C)/C(=C\C(C)C)CN([As])c2ccccc21</smiles>

$4 a$

White solid; m.p.: $83-84{ }^{\circ} \mathrm{C}$; $(37 \mathrm{mg} ; 89 \%$ yield; 95\% ee); [The ee value was determined by chiral HPLC using a Chiralpak IC-3 column with hexane : isopropanol $=95: 5$ at a flow rate $0.7 \mathrm{~mL} / \mathrm{min}$ detected at $214 \mathrm{~nm}$ wavelength. Elution time: $\mathrm{t}($ minor $)=10.6 \mathrm{~min} ; \mathrm{t}($ major $)=11.3 \mathrm{~min} ;[\alpha]_{\mathrm{D}}{ }^{29}=12.23\left(\mathrm{c}=1.0\right.$, in $\left.\left.\mathrm{CDCl}_{3}\right)\right] .{ }^{1} \mathbf{H}$ NMR (400 MHz, CDCl $\left.)_{3}\right): \delta .79(\mathrm{dd}, J=8.0 \mathrm{~Hz}, 1.2 \mathrm{~Hz}, 1 \mathrm{H}), 7.31(\mathrm{~d}, J=8.4 \mathrm{~Hz}$, 2H), $7.20(\mathrm{td}, J=7.2 \mathrm{~Hz}, 1.6 \mathrm{~Hz}, 1 \mathrm{H}), 7.13(\mathrm{td}, J=7.6 \mathrm{~Hz}, 1.2 \mathrm{~Hz}, 1 \mathrm{H}), 7.08-7.04(\mathrm{~m}$, $3 \mathrm{H}), 5.37(\mathrm{~s}, 1 \mathrm{H}), 4.99-4.90(\mathrm{~m}, 2 \mathrm{H}), 4.83-4.77(\mathrm{~m}, 1 \mathrm{H}), 4.65(\mathrm{~d}, J=15.2 \mathrm{~Hz}, 1 \mathrm{H})$, $4.37(\mathrm{~d}, J=15.2 \mathrm{~Hz}, 1 \mathrm{H}), 2.32(\mathrm{~s}, 3 \mathrm{H}), 1.10(\mathrm{~s}, 3 \mathrm{H}), 0.26(\mathrm{~s}, 9 \mathrm{H}) ;{ }^{13} \mathbf{C}$ NMR $(\mathbf{1 0 0}$ MHz, $\left.\mathbf{C D C l}_{3}\right): \delta 152.6,144.4,143.5,137.1,136.0,135.8,129.4,129.0,128.1,126.9$, 126.7, 126.1, 125.4, 112.3, 49.3, 47.9, 28.7, 21.5, 0.2; IR (neat, $\mathbf{~ c m}^{-1}$ ): v 2953, 2900 , $1618,1482,1344,1250,1160,839,756,668$; HRMS calculated for $\mathrm{C}_{23} \mathrm{H}_{30} \mathrm{NO}_{2} \mathrm{SSi}$ $(\mathrm{M}+\mathrm{H})^{+}:$412.1761; Found: 412.1753. 


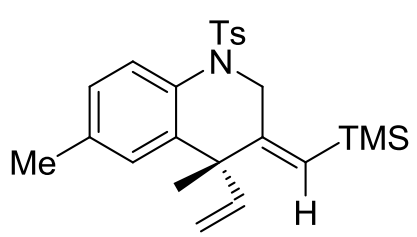

4b

White solid; m.p.: $82-83{ }^{\circ} \mathrm{C}$; $(31 \mathrm{mg} ; 73 \%$ yield; 95\% ee); [The ee value was determined by chiral HPLC using a Chiralpak IC-3 column with hexane : isopropanol $=95: 5$ at a flow rate $0.7 \mathrm{~mL} / \mathrm{min}$ detected at $214 \mathrm{~nm}$ wavelength. Elution time: $\mathrm{t}($ minor $)=11.1 \mathrm{~min} ; \mathrm{t}($ major $)=11.7 \mathrm{~min} ;[\alpha]_{\mathrm{D}}{ }^{30}=4.91\left(\mathrm{c}=1.0\right.$, in $\left.\left.\mathrm{CDCl}_{3}\right)\right] .{ }^{\mathbf{1}} \mathbf{H} \mathbf{~ N M R}$ $\left(400 \mathrm{MHz}, \mathbf{C D C l}_{3}\right): \delta 7.67(\mathrm{~d}, J=8.0 \mathrm{~Hz}, 1 \mathrm{H}), 7.32(\mathrm{~d}, J=8.4 \mathrm{~Hz}, 2 \mathrm{H}), 7.07$ (d, $J=$ $7.6 \mathrm{~Hz}, 2 \mathrm{H}), 7.01(\mathrm{~d}, J=8.4 \mathrm{~Hz}, 1 \mathrm{H}), 6.83(\mathrm{~s}, 1 \mathrm{H}), 5.35(\mathrm{~s}, 1 \mathrm{H}), 4.99-4.90(\mathrm{~m}, 2 \mathrm{H})$, 4.82-4.75 (m, 1H), $4.63(\mathrm{~d}, J=15.2 \mathrm{~Hz}, 1 \mathrm{H}), 4.33(\mathrm{~d}, J=15.6 \mathrm{~Hz}, 1 \mathrm{H}), 2.32(\mathrm{~s}, 3 \mathrm{H})$, 2.27 (s, 3H), 1.08 (s, 3H), 0.25 (s, 9H); ${ }^{13} \mathbf{C}$ NMR (100 MHz, CDCl $): \delta$ 152.8, 144.4, 143.3, 136.8, 135.9, 135.7, 133.4, 129.7, 129.0, 128.1, 127.6, 126.8, 125.2, 112.1, 49.4, 47.8, 28.7, 21.5, 21.2, 0.2; IR (neat, $\mathbf{~ c m}^{-1}$ ): $v$ 2945, 1623, 1492, 1346, 1159, 1045, 914, 848, 668; HRMS calculated for $\mathrm{C}_{24} \mathrm{H}_{32} \mathrm{NO}_{2} \mathrm{SSi}(\mathrm{M}+\mathrm{H})^{+}$: 426.1918; Found: 426.1906 .

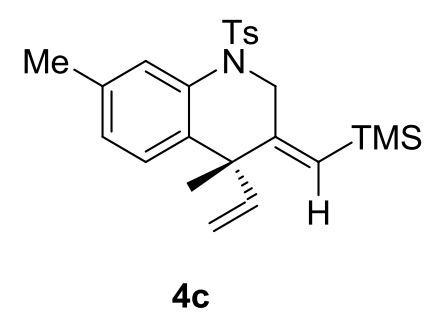

White solid; m.p.: $103-104{ }^{\circ} \mathrm{C}$; (30 mg; 70\% yield; 93\% ee); [The ee value was determined by chiral HPLC using a Chiralpak IC-3 column with hexane : isopropanol $=95: 5$ at a flow rate $0.7 \mathrm{~mL} / \mathrm{min}$ detected at $214 \mathrm{~nm}$ wavelength. Elution time: $\mathrm{t}($ minor $)=7.9 \mathrm{~min} ; \mathrm{t}($ major $)=9.0 \mathrm{~min} ;[\alpha]_{\mathrm{D}}{ }^{30}=-9.32\left(\mathrm{c}=1.0\right.$, in $\left.\left.\mathrm{CDCl}_{3}\right)\right] .{ }^{\mathbf{1}} \mathbf{H} \mathbf{~ N M R}$ (400 MHz, $\left.\mathbf{C D C l}_{3}\right): \delta 7.61(\mathrm{~s}, 1 \mathrm{H}), 7.33(\mathrm{~d}, J=8.4 \mathrm{~Hz}, 2 \mathrm{H}), 7.07(\mathrm{~d}, J=8.0 \mathrm{~Hz}, 2 \mathrm{H})$, 6.96-6.91 (m, 2H), $5.36(\mathrm{~s}, 1 \mathrm{H}), 4.96-4.87(\mathrm{~m}, 2 \mathrm{H}), 4.84-4.77(\mathrm{~m}, 1 \mathrm{H}), 4.62(\mathrm{~d}, J=$ $15.2 \mathrm{~Hz}, 1 \mathrm{H}), 4.34$ (d, $J=15.2 \mathrm{~Hz}, 1 \mathrm{H}), 2.35$ (s, 3H), 2.32 (s, 3H), 1.08 (s, 3H), 0.25 $(\mathrm{s}, 9 \mathrm{H}) ;{ }^{13} \mathbf{C}$ NMR (100 MHz, $\left.\mathbf{C D C l}_{3}\right): \delta$ 152.8, 144.6, 143.4, 136.5, 135.9, 135.8, 134.1, 129.1, 129.0, 128.1, 127.1, 126.7, 125.6, 112.0, 49.4, 47.6, 28.7, 21.5, 21.2, 0.2; 
IR (neat, $\mathbf{c m}^{-1}$ ): $v$ 2948, 1623, 1342, 1248, 1157, 1051, 831, 669; HRMS calculated for $\mathrm{C}_{24} \mathrm{H}_{32} \mathrm{NO}_{2} \mathrm{SSi}(\mathrm{M}+\mathrm{H})^{+}$: 426.1918; Found: 426.1906 .<smiles>C=C[C@]1(C)/C(=C\C(C)(C)C)CN([As])c2ccc(OC)cc21</smiles>

4d

White solid; m.p.: $100-101{ }^{\circ} \mathrm{C}$; (34 mg; 76\% yield; 93\% ee); [The ee value was determined by chiral HPLC using a Chiralpak IG column with hexane $:$ isopropanol = $95: 5$ at a flow rate $0.7 \mathrm{~mL} / \mathrm{min}$ detected at $214 \mathrm{~nm}$ wavelength. Elution time: $\mathrm{t}$ (minor) $=15.3$ min; $\mathrm{t}($ major $)=16.2 \mathrm{~min} ;[\alpha]_{\mathrm{D}}{ }^{29}=-1.89\left(\mathrm{c}=1.0\right.$ in $\left.\left.\mathrm{CDCl}_{3}\right)\right] .{ }^{\mathbf{1}} \mathbf{H}$ NMR $(400$ MHz, $\left.\mathbf{C D C l}_{3}\right): \delta 7.82(\mathrm{~d}, J=9.2 \mathrm{~Hz}, 1 \mathrm{H}), 7.28(\mathrm{~d}, J=8.4 \mathrm{~Hz}, 2 \mathrm{H}), 7.07(\mathrm{~d}, J=8.0$ $\mathrm{Hz}, 2 \mathrm{H}), 6.77(\mathrm{dd}, J=8.8 \mathrm{~Hz}, 3.2 \mathrm{~Hz}, 1 \mathrm{H}), 6.56(\mathrm{~d}, J=3.2 \mathrm{~Hz}, 1 \mathrm{H}), 5.34(\mathrm{~s}, 1 \mathrm{H})$, 4.98-4.88 (m, 2H), 4.78-4.71 (m, 1H), $4.60(\mathrm{~d}, J=15.2 \mathrm{~Hz}, 1 \mathrm{H}), 4.34(\mathrm{~d}, J=15.6 \mathrm{~Hz}$, 1H), 3.76 (s, 3H), 2.32 (s, 3H), 1.05 (s, 3H), 0.25 (s, 9H); ${ }^{13}$ C NMR (100 MHz, $\left.\mathbf{C D C l}_{3}\right): \delta 157.7,152.4,144.3,143.3,138.8,135.7,129.0,128.2,127.1,126.9,114.6$, 112.2, 112.1, 55.4, 49.5, 48.1, 28.8, 21.5, 0.2; IR (neat, $\mathbf{~ c m}^{-1}$ ): $v 2952,1604,1490$, 1346, 1158, 1043, 845, 668, 543; HRMS calculated for $\mathrm{C}_{24} \mathrm{H}_{32} \mathrm{NO}_{3} \mathrm{SSi}(\mathrm{M}+\mathrm{H})^{+}$: 442.1867; Found: 442.1854.<smiles>C=C[C@]1(C)/C(=C\[As](C)C)CN([As])c2ccc(F)cc21</smiles>

$4 e$

White solid; m.p.: $79-80{ }^{\circ} \mathrm{C}$; $(31 \mathrm{mg} ; 72 \%$ yield; $94 \%$ ee); [The ee value was determined by chiral HPLC using a Chiralpak IC-3 column with hexane : isopropanol $=95: 5$ at a flow rate $0.7 \mathrm{~mL} / \mathrm{min}$ detected at $214 \mathrm{~nm}$ wavelength. Elution time: $\mathrm{t}($ minor $)=7.4$ min; $\mathrm{t}($ major $)=7.7 \mathrm{~min} ;[\alpha]_{\mathrm{D}}{ }^{30}=6.98\left(\mathrm{c}=1.0\right.$, in $\left.\left.\mathrm{CDCl}_{3}\right)\right] .{ }^{\mathbf{1}} \mathbf{H} \mathbf{~ N M R}$ (400 MHz, $\left.\mathbf{C D C l}_{3}\right): \delta 7.77(\mathrm{dd}, J=9.2 \mathrm{~Hz}, 5.6 \mathrm{~Hz}, 1 \mathrm{H}), 7.30(\mathrm{~d}, J=8.0 \mathrm{~Hz}, 2 \mathrm{H})$, $7.09(\mathrm{~d}, J=8.4 \mathrm{~Hz}, 2 \mathrm{H}), 6.94-6.89(\mathrm{~m}, 1 \mathrm{H}), 6.76(\mathrm{dd}, J=10.0 \mathrm{~Hz}, 3.2 \mathrm{~Hz}, 1 \mathrm{H}), 5.37$ 
(s, 1H), 4.99-4.92 (m, 2H), 4.81-4.74 (m, 1H), $4.61(\mathrm{~d}, J=15.2 \mathrm{~Hz}, 1 \mathrm{H}), 4.36$ (d, $J=$ $15.6 \mathrm{~Hz}, 1 \mathrm{H}), 2.33$ (s, 3H), 1.06 (s, 3H), 0.26 (s, 9H); ${ }^{13} \mathbf{C}$ NMR (100 MHz, $\left.\mathbf{C D C l}_{3}\right)$ : $\delta 160.8(\mathrm{~d}, J=243.6 \mathrm{~Hz}), 151.7,143.8,143.7,139.7(\mathrm{~d}, J=6.8 \mathrm{~Hz}), 135.6,132.0(\mathrm{~d}$, $J=2.2 \mathrm{~Hz}), 129.1,128.2,127.5,127.4(\mathrm{~d}, J=8.3 \mathrm{~Hz}), 115.7(\mathrm{~d}, J=23.5 \mathrm{~Hz}), 114.0$ (d, $J=22.8 \mathrm{~Hz}$ ), 112.7, 49.4, 48.1, 28.7, 21.5, 0.2; IR (neat, $\mathbf{c m}^{-1}$ ): $v$ 2961, 2922 , 1485, 1350, 1252, 1159, 1051, 836, 670; HRMS calculated for $\mathrm{C}_{23} \mathrm{H}_{29} \mathrm{FNO}_{2} \mathrm{SSi}$ $(\mathrm{M}+\mathrm{H})^{+}:$430.1667; Found: 430.1665.

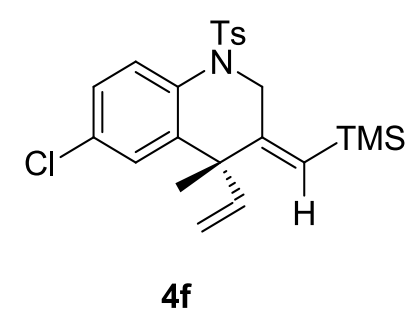

White solid; m.p.: $85-86{ }^{\circ} \mathrm{C}$; $(33 \mathrm{mg} ; 75 \%$ yield; $95 \%$ ee); [The ee value was determined by chiral HPLC using a Chiralpak IG column with hexane : isopropanol = $95: 5$ at a flow rate $0.7 \mathrm{~mL} / \mathrm{min}$ detected at $214 \mathrm{~nm}$ wavelength. Elution time: $\mathrm{t}$ (minor) $=8.9$ min; $\mathrm{t}($ major $)=9.5 \mathrm{~min} ;[\alpha]_{\mathrm{D}}{ }^{30}=0.95\left(\mathrm{c}=1.0\right.$, in $\left.\left.\mathrm{CDCl}_{3}\right)\right] .{ }^{\mathbf{1}} \mathbf{H} \mathbf{N M R}(\mathbf{4 0 0} \mathbf{M H z}$ $\left.\mathbf{C D C l}_{3}\right): \delta 7.75(\mathrm{~d}, J=8.8 \mathrm{~Hz}, 1 \mathrm{H}), 7.33(\mathrm{~d}, J=8.4 \mathrm{~Hz}, 2 \mathrm{H}), 7.17(\mathrm{dd}, J=8.8 \mathrm{~Hz}, 2.4$ $\mathrm{Hz}, 1 \mathrm{H}), 7.10(\mathrm{~d}, J=8.0 \mathrm{~Hz}, 2 \mathrm{H}), 7.02$ (d, $J=2.4 \mathrm{~Hz}, 1 \mathrm{H}), 5.38$ (s, 1H), 5.00-4.94 (m, 2H), 4.86-4.79 (m, 1H), $4.61(\mathrm{~d}, J=15.2 \mathrm{~Hz}, 1 \mathrm{H}), 4.36(\mathrm{~d}, J=15.2 \mathrm{~Hz}, 1 \mathrm{H}), 2.34$ (s, 3H), 1.08 (s, 3H), 0.25 (s, 9H); ${ }^{13}$ C NMR (100 MHz, $\left.\mathbf{C D C l}_{3}\right): \delta 151.7,143.8,143.7$, 139.0, 135.6, 134.7, 131.5, 129.3, 129.2, 128.1, 127.5, 127.0, 126.6, 113.0, 49.2, 48.0, 28.6, 21.5, 0.2; IR (neat, $\mathbf{c m}^{-1}$ ): $v$ 2956, 1601, 1350, 1251, 1160, 1088, 1048, 845, 664; HRMS calculated for $\mathrm{C}_{23} \mathrm{H}_{32} \mathrm{ClN}_{2} \mathrm{O}_{2} \mathrm{SSi}\left(\mathrm{M}+\mathrm{NH}_{4}\right)^{+}$: 463.1637; Found: 463.1629. 
<smiles>C=C[C@]1(C)/C(=C/[15C](C)(C)C)CN([As])c2ccc(Br)cc21</smiles>

4g

White solid; m.p.: $91-92{ }^{\circ} \mathrm{C}$; $(27 \mathrm{mg} ; 55 \%$ yield; 92\% ee); [The ee value was determined by chiral HPLC using a Chiralpak IG column with hexane : isopropanol = $98: 2$ at a flow rate $0.7 \mathrm{~mL} / \mathrm{min}$ detected at $214 \mathrm{~nm}$ wavelength. Elution time: $\mathrm{t}($ minor) $=15.1 \mathrm{~min} ; \mathrm{t}($ major $)=15.9 \mathrm{~min} ;[\alpha]_{\mathrm{D}}{ }^{30}=-1.36\left(\mathrm{c}=1.0\right.$, in $\left.\left.\mathrm{CDCl}_{3}\right)\right] .{ }^{\mathbf{1}} \mathbf{H}$ NMR (400 MHz, $\left.\mathbf{C D C l}_{3}\right): \delta 7.69(\mathrm{~d}, J=8.8 \mathrm{~Hz}, 1 \mathrm{H}), 7.35(\mathrm{~d}, J=8.4 \mathrm{~Hz}, 2 \mathrm{H}), 7.31(\mathrm{dd}, J=8.8$ $\mathrm{Hz}, 2.0 \mathrm{~Hz}, 1 \mathrm{H}), 7.16$ (d, $J=2.0 \mathrm{~Hz}, 1 \mathrm{H}), 7.12$ (d, $J=8.4 \mathrm{~Hz}, 2 \mathrm{H}), 5.39$ (s, 1H), 5.00-4.95 (m, 2H), 4.89-4.82 (m, 1H), $4.60(\mathrm{~d}, J=15.6 \mathrm{~Hz}, 1 \mathrm{H}), 4.36(\mathrm{~d}, J=15.2 \mathrm{~Hz}$, 1H), 2.34 (s, 3H), 1.09 (s, 3H), 0.25 (s, 9H); ${ }^{13} \mathbf{C}$ NMR (100 MHz, CDCl 3 ): $\delta$ 151.7, $143.8,143.7,139.3,135 . .6,135.2,132.2,129.9,129.2,128.0,127.5,126.9,119.4$, 113.0, 49.2, 48.0, 28.6, 21.5, 0.2; IR (neat, $\mathbf{c m}^{-1}$ ): $v$ 2953, 1603, 1472, 1350, 1159, 1085, 1047, 844, 662; HRMS calculated for $\mathrm{C}_{23} \mathrm{H}_{29} \mathrm{BrNO}_{2} \mathrm{SSi}(\mathrm{M}+\mathrm{H})^{+}$: 490.0866; Found: 490.0866 .<smiles>C=C[C@]1(C)/C(=C/C(C)(C)C)CN([As])c2ccc(C(F)(F)F)cc21</smiles>

4h

White solid; m.p.: $96-97{ }^{\circ} \mathrm{C}$; $(27 \mathrm{mg} ; 56 \%$ yield; $95 \%$ ee); [The ee value was determined by chiral HPLC using a Chiralpak IC-3 column with hexane : isopropanol $=97: 3$ at a flow rate $0.7 \mathrm{~mL} / \mathrm{min}$ detected at $214 \mathrm{~nm}$ wavelength. Elution time: $\mathrm{t}($ minor $)=5.1 \mathrm{~min} ; \mathrm{t}($ major $)=5.3 \mathrm{~min} ;[\alpha]_{\mathrm{D}}{ }^{29}=0.77\left(\mathrm{c}=1.0\right.$, in $\left.\left.\mathrm{CDCl}_{3}\right)\right] .{ }^{1} \mathbf{H} \mathbf{~ N M R}$ (400 MHz, $\left.\mathbf{C D C l}_{3}\right): \delta 8.08(\mathrm{~s}, 1 \mathrm{H}), 7.38-7.33(\mathrm{~m}, 3 \mathrm{H}), 7.19(\mathrm{~d}, J=8.4 \mathrm{~Hz}, 1 \mathrm{H}), 7.11$ $(\mathrm{d}, J=8.0 \mathrm{~Hz}, 2 \mathrm{H}), 5.44(\mathrm{~s}, 1 \mathrm{H}), 5.02-4.95(\mathrm{~m}, 3 \mathrm{H}), 4.63(\mathrm{~d}, J=15.2 \mathrm{~Hz}, 1 \mathrm{H}), 4.41$ (d, $J=15.2 \mathrm{~Hz}, 1 \mathrm{H}), 2.34(\mathrm{~s}, 3 \mathrm{H}), 1.14(\mathrm{~s}, 3 \mathrm{H}), 0.26(\mathrm{~s}, 9 \mathrm{H}) ;{ }^{\mathbf{1 3}} \mathbf{C}$ NMR (100 MHz, $\left.\mathbf{C D C l}_{3}\right): \delta 151.7,144.0,143.7,140.8,136.7,135.6,130.0,129.3,129.1$ (q, $J=33.0$ $\mathrm{Hz}), 128.1,127.6,123.8$ (q, $J=270.5 \mathrm{~Hz}), 122.2$ (q, $J=3.1 \mathrm{~Hz}), 122.0$ (q, $J=4.6 \mathrm{~Hz})$, 
113.2, 49.1, 48.1, 28.4, 21.5, 0.2; IR (neat, $\mathbf{c m}^{-1}$ ): $v$ 2950, 1622, 1414, 1325, 1161, 1127, 1048, 897, 836, 701, 578; HRMS calculated for $\mathrm{C}_{24} \mathrm{H}_{32} \mathrm{~F}_{3} \mathrm{~N}_{2} \mathrm{O}_{2} \mathrm{SSi}\left(\mathrm{M}+\mathrm{NH}_{4}\right)^{+}$: 497.1900; Found: 497.1892.

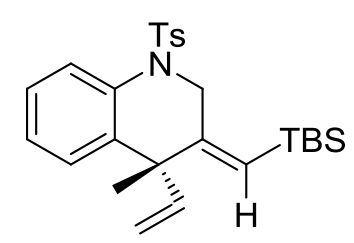

$4 \mathbf{i}$

White solid; m.p.: $105-106{ }^{\circ} \mathrm{C}$; $(20 \mathrm{mg} ; 44 \%$ yield; $82 \%$ ee); [The ee value was determined by chiral HPLC using a Chiralpak IG column with hexane : isopropanol = $95: 5$ at a flow rate $0.7 \mathrm{~mL} / \mathrm{min}$ detected at $214 \mathrm{~nm}$ wavelength. Elution time: $\mathrm{t}$ (minor) $=9.0 \mathrm{~min} ; \mathrm{t}($ major $)=10.1 \mathrm{~min} ;[\alpha]_{\mathrm{D}}{ }^{29}=6.40\left(\mathrm{c}=1.0\right.$, in $\left.\left.\mathrm{CDCl}_{3}\right)\right] .{ }^{\mathbf{1}} \mathbf{H} \mathbf{~ N M R}(\mathbf{4 0 0}$ MHz, $\left.\mathbf{C D C l}_{3}\right): \delta 7.79(\mathrm{dd}, J=8.0 \mathrm{~Hz}, 0.8 \mathrm{~Hz}, 1 \mathrm{H}), 7.31(\mathrm{~d}, J=8.4 \mathrm{~Hz}, 2 \mathrm{H})$, 7.20-7.13 (m, 2H), 7.07-7.05 (m, 3H), $5.43(\mathrm{~s}, 1 \mathrm{H}), 4.99-4.91(\mathrm{~m}, 2 \mathrm{H}), 4.83-4.79(\mathrm{~m}$, 1H), $4.63(\mathrm{~d}, J=15.6 \mathrm{~Hz}, 1 \mathrm{H}), 4.36(\mathrm{~d}, J=15.6 \mathrm{~Hz}, 1 \mathrm{H}), 2.32(\mathrm{~s}, 3 \mathrm{H}), 1.11$ (s, 3H), 0.86 (s, 9H), 0.33 (s, 3H), 0.27 (s, 3H); ${ }^{13}$ C NMR (100 MHz, CDCl $): \delta$ 153.6, 144.4, $143.5,137.2$, 136.1, 135.9, 129.3, 129.0, 128.1, 126.7, 126.1, 125.5, 124.4, 122.2, 49.3, 48.2, 28.7, 26.5, 21.5, 17.5, -4.3, -4.4; IR (neat, $\mathbf{c m}^{-1}$ ): v 2925, 2853, 1621, 1347, 1161, 807, 777, 664; HRMS calculated for $\mathrm{C}_{26} \mathrm{H}_{36} \mathrm{NO}_{2} \mathrm{SSi}(\mathrm{M}+\mathrm{H})^{+}$: 454.2231; Found: 454.2218 .<smiles>C=C[C@]1(C)/C(=C/C(C)(C)C)CN([As])c2ccccc21</smiles>

$4 \mathbf{j}$

Pale yellow solid; m.p.: $87-88^{\circ} \mathrm{C}$; $(27 \mathrm{mg} ; 68 \%$ yield; $78 \%$ ee $)$; [The ee value was determined by chiral HPLC using a Chiralpak IG column with hexane : isopropanol = $95: 5$ at a flow rate $0.7 \mathrm{~mL} / \mathrm{min}$ detected at $214 \mathrm{~nm}$ wavelength. Elution time: $\mathrm{t}$ (minor) $=12.8 \mathrm{~min} ; \mathrm{t}$ (major) $=15.8 \mathrm{~min} ;[\alpha]_{\mathrm{D}}{ }^{29}=2.31\left(\mathrm{c}=1.0\right.$, in $\left.\left.\mathrm{CDCl}_{3}\right)\right] .{ }^{\mathbf{1}} \mathbf{H} \mathbf{N M R}(\mathbf{4 0 0}$ MHz, $\left.\mathbf{C D C l}_{3}\right): \delta 7.77(\mathrm{~d}, J=8.0 \mathrm{~Hz}, 1 \mathrm{H}), 7.39(\mathrm{~d}, J=8.4 \mathrm{~Hz}, 2 \mathrm{H}), 7.20-7.15(\mathrm{~m}, 1 \mathrm{H})$, 
7.12-7.06 (m, 4H), $5.23(\mathrm{~s}, 1 \mathrm{H}), 5.01-4.85(\mathrm{~m}, 4 \mathrm{H}), 4.41(\mathrm{~d}, J=15.2 \mathrm{~Hz}, 1 \mathrm{H}), 2.33(\mathrm{~s}$, 3H), 1.21 (s, 9H), 1.13 (s, 3H); ${ }^{13}$ C NMR (100 MHz, $\left.\mathbf{C D C l}_{\mathbf{3}}\right): \delta 145.0,143.5,138.2$, $136.9,136.3,136.1,134.0,129.3,129.1,127.8,126.6,125.8,124.7,111.7,46.2,45.3$, 33.3, 31.3, 29.4, 21.5; IR (neat, $\mathbf{c m}^{-1}$ ): v 2947, 1477, 1338, 1157, 1052, 908, 757, 672; HRMS calculated for $\mathrm{C}_{24} \mathrm{H}_{30} \mathrm{NO}_{2} \mathrm{~S}(\mathrm{M}+\mathrm{H})^{+}:$396.1992; Found: 396.1982 .

\section{Transformation of Compound 2a}

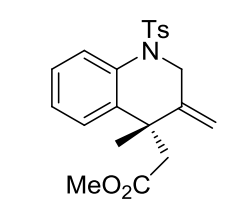

$5 a$ $73 \%$ yield, $93 \%$ ee

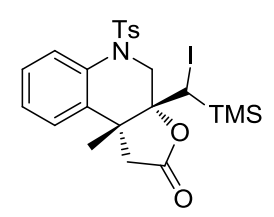

$8 \mathbf{a}$

$78 \%$ yield, $91 \%$ ee
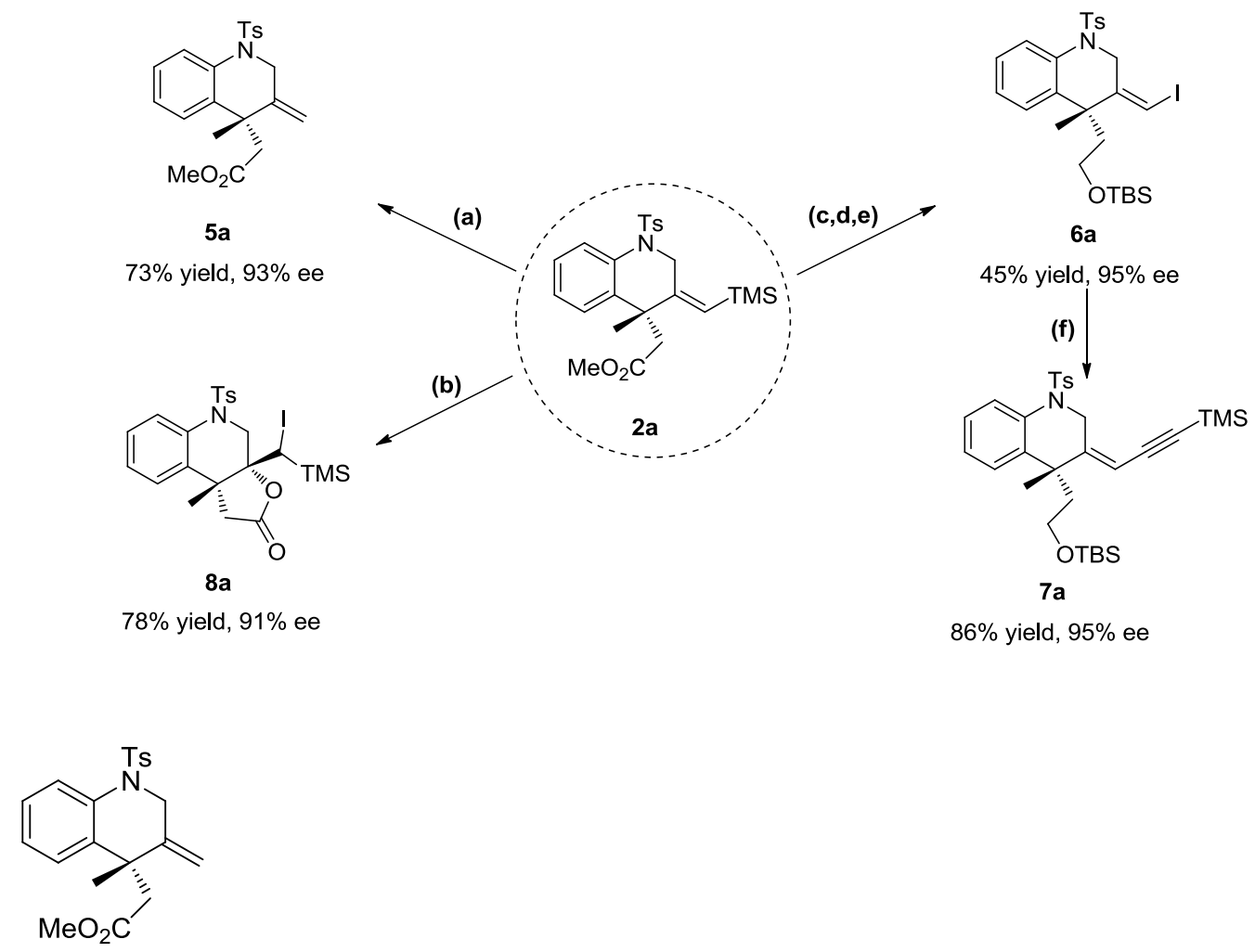

$5 \mathbf{a}$

In a $25 \mathrm{~mL}$ single neck flask, $2 \mathbf{a}$ (1.0 mmol, 1.0 equiv.) was dissolved in anhydrous THF ( $8 \mathrm{~mL})$. Then TBAF ( $2 \mathrm{~mL}, 1 \mathrm{M}$ in THF, 2.0 equiv.) was added to the mixture and the resulting solution was stirred at $50{ }^{\circ} \mathrm{C}$. After completion of the reaction, solvent was evaporated under reduced pressure, the residue was purified by flash column chromatography (petroleum ether : ethyl acetate $=5: 1$ ) to obtain the compound 5a.

White solid; m.p.: $120-121{ }^{\circ} \mathrm{C}$; $(281 \mathrm{mg} ; 73 \%$ yield; $93 \%$ ee); [The ee value was determined by chiral HPLC using a Chiralpak IC-3 column with hexane : isopropanol $=70: 30$ at a flow rate $0.7 \mathrm{~mL} / \mathrm{min}$ detected at $214 \mathrm{~nm}$ wavelength. Elution time: 
$\mathrm{t}($ minor $)=13.9 \mathrm{~min} ; \mathrm{t}($ major $)=18.0 \mathrm{~min} ;[\alpha]_{\mathrm{D}}{ }^{29}=-58.53\left(\mathrm{c}=1.0\right.$, in $\left.\left.\mathrm{CDCl}_{3}\right)\right] .{ }^{\mathbf{1}} \mathbf{H}$ NMR (400 MHz, CDCl $): \delta 7.81(\mathrm{~d}, J=8.0 \mathrm{~Hz}, 1 \mathrm{H}), 7.35(\mathrm{~d}, J=8.4 \mathrm{~Hz}, 2 \mathrm{H})$, 7.25-7.21 (m, 1H), $7.19(\mathrm{~d}, J=4.0 \mathrm{~Hz}, 2 \mathrm{H}), 7.09(\mathrm{~d}, J=8.0 \mathrm{~Hz}, 2 \mathrm{H}), 5.07(\mathrm{~s}, 1 \mathrm{H})$, $4.84(\mathrm{~s}, 1 \mathrm{H}), 4.63(\mathrm{~d}, J=14.8 \mathrm{~Hz}, 1 \mathrm{H}), 4.22(\mathrm{~d}, J=15.2 \mathrm{~Hz}, 1 \mathrm{H}), 3.48$ (s, 3H), 2.67 (d, $J=14.4 \mathrm{~Hz}, 1 \mathrm{H}), 2.47(\mathrm{~d}, J=14.4 \mathrm{~Hz}, 1 \mathrm{H}), 2.31(\mathrm{~s}, 3 \mathrm{H}), 0.88(\mathrm{~s}, 3 \mathrm{H}) ;{ }^{13} \mathbf{C}$ NMR $(100$ MHz, $\left.\mathbf{C D C l}_{3}\right): \delta 170.8,144.8,143.5,137.7,136.2,136.1,129.1,128.1,127.0,126.6$, 126.4, 126.3, 112.9, 51.9, 51.4, 48.4, 40.4, 27.9, 21.5; IR (neat, $\mathbf{~ c m}^{-1}$ ): v 2948, 2921, 2850, 1741, 1486, 1345, 1155, 1073, 1013, 877, 763, 668; HRMS calculated for $\mathrm{C}_{21} \mathrm{H}_{27} \mathrm{~N}_{2} \mathrm{O}_{4} \mathrm{~S}\left(\mathrm{M}+\mathrm{NH}_{4}\right)^{+}$: 403.1686; Found: 403.1683.

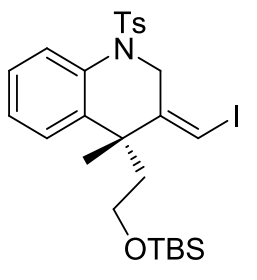

$6 a$

In a $25 \mathrm{~mL}$ two neck flask, $2 \mathbf{a}(1.0 \mathrm{mmol}, 1.0$ equiv.) was dissolved in anhydrous THF $(7 \mathrm{~mL})$. The mixture was cooled to $-20{ }^{\circ} \mathrm{C}$, then DIBAL-H $(3 \mathrm{~mL}, 1 \mathrm{M}$ in hexane, 3.0 equiv.) was added to the reaction mixture, followed by stirring for additional half an hour at the same temperature. After completion of the reaction, the mixture was quenched by water $(10 \mathrm{~mL})$. The mixture was filtered by celite, washed with ethyl acetate to remove the aluminium salt. The organic layer was dried over anhydrous $\mathrm{Na}_{2} \mathrm{SO}_{4}$, and then evaporated under reduced pressure. The residue was dissolved in DCM (10 mL), then TBSCl (1.2 mmol, 1.2 equiv.) and TEA(1 mL) were added sequentially. The reaction was stirred at room temperature overnight. After completion of the reaction, the mixture was filtered by a short silica column, the solvent was evaporated under reduced pressure. The residue was dissolved in $\mathrm{CH}_{3} \mathrm{CN}$ $(10 \mathrm{~mL})$, then NIS (1.5 mmol, 1.5 equiv.) was added to the mixture and the resulting solution was stirred at room temperature overnight (avoid light). After completion of the reaction, the solvent was evaporated under reduced pressure, the residue was purified by flash column chromatography (petroleum ether : ethyl acetate $=5: 1$ ) to obtain the compound $\mathbf{6 a}$. 
White solid; m.p.: $109-111^{\circ} \mathrm{C}$; $(268 \mathrm{mg} ; 45 \%$ yield for three steps; $95 \%$ ee $)$; [The ee value was determined by chiral HPLC using a Chiralpak IF-3 column with hexane : isopropanol $=98: 2$ at a flow rate $0.7 \mathrm{~mL} / \mathrm{min}$ detected at $214 \mathrm{~nm}$ wavelength. Elution time: $\mathrm{t}($ minor $)=12.7 \mathrm{~min} ; \mathrm{t}($ major $)=14.2 \mathrm{~min} ;[\alpha]_{\mathrm{D}}{ }^{28}=-170.41(\mathrm{c}=1.0$, in $\left.\left.\mathrm{CDCl}_{3}\right)\right] .{ }^{1} \mathbf{H}$ NMR (400 MHz, $\left.\mathbf{C D C l}_{3}\right): \delta 7.85(\mathrm{~d}, J=7.6 \mathrm{~Hz}, 1 \mathrm{H}), 7.48(\mathrm{~d}, J=8.4 \mathrm{~Hz}$, 2H), 7.29-7.22 (m, 3H), $7.14(\mathrm{~d}, J=8.4 \mathrm{~Hz}, 2 \mathrm{H}), 6.20(\mathrm{~s}, 1 \mathrm{H}), 4.99$ (d, $J=16.0 \mathrm{~Hz}$, $1 \mathrm{H}), 4.12(\mathrm{dd}, J=16.0 \mathrm{~Hz}, 1.2 \mathrm{~Hz}, 1 \mathrm{H}), 3.20-3.13(\mathrm{~m}, 2 \mathrm{H}), 2.34(\mathrm{~s}, 3 \mathrm{H}), 2.04-1.97(\mathrm{~m}$, $1 \mathrm{H}), 1.80-1.73(\mathrm{~m}, 1 \mathrm{H}), 0.83(\mathrm{~s}, 3 \mathrm{H}), 0.82(\mathrm{~s}, 9 \mathrm{H}),-0.05(\mathrm{~s}, 3 \mathrm{H}),-0.06(\mathrm{~s}, 3 \mathrm{H}) ;{ }^{13} \mathrm{C}$ NMR (100 MHz, $\left.\mathbf{C D C l}_{3}\right): \delta$ 147.6, 143.7, 137.5, 136.4, 136.1, 129.2, 127.9, 126.9, $126.6,126.4,125.9,78.3,59.7,53.5,47.4,43.8,29.7,26.0,21.6,18.2,-5.2,-5.2$; IR $\left(\right.$ neat, $\left.\mathbf{c m}^{-1}\right): \vee 2951,2928,2855,1597,1484,1354,1252,1162,1087,834,765,661$; HRMS calculated for $\mathrm{C}_{26} \mathrm{H}_{40} \mathrm{IN}_{2} \mathrm{O}_{3} \mathrm{SSi}\left(\mathrm{M}+\mathrm{NH}_{4}\right)^{+}$: 615.1568; Found: 615.1556.

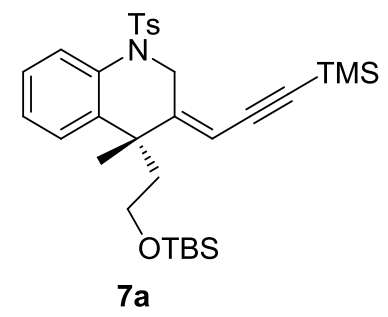

In a $25 \mathrm{~mL}$ two neck flask, $6 \mathbf{a}(0.5 \mathrm{mmol}, 1.0$ equiv.) was dissolved in TEA (10 $\mathrm{mL})$, then ethynyltrimethylsilane (0.6 mmol, 1.2 equiv.), $\mathrm{PdCl}_{2}\left(\mathrm{PPh}_{3}\right)_{2}(0.005 \mathrm{mmol}$, 0.01 equiv. $)$ and $\mathrm{CuI}(0.005 \mathrm{mmol}, 0.01$ equiv. $)$ were added to the mixture sequentially. The resulting solution was stirred at $-20{ }^{\circ} \mathrm{C}$ for $2 \mathrm{~h}$. After completion of the reaction, the mixture was filtered by celite, washed with ethyl acetate. The solvent was evaporated under reduced pressure, the residue was purified by flash column chromatography (petroleum ether : ethyl acetate $=15: 1-10: 1$ ) to obtain the compound $7 \mathbf{a}$.

Pale yellow solid; m.p.: $101-102{ }^{\circ} \mathrm{C}$; $(244 \mathrm{mg} ; 86 \%$ yield; $95 \%$ ee); [The ee value was determined by chiral HPLC using a Chiralpak AY-3 column with hexane : isopropanol $=98: 2$ at a flow rate $0.7 \mathrm{~mL} / \mathrm{min}$ detected at $214 \mathrm{~nm}$ wavelength. Elution time: $\mathrm{t}($ minor $)=5.1 \mathrm{~min} ; \mathrm{t}$ (major $)=6.1 \mathrm{~min} ;[\alpha]_{\mathrm{D}}{ }^{27}=-205.92\left(\mathrm{c}=1.0\right.$, in $\left.\left.\mathrm{CDCl}_{3}\right)\right] .{ }^{1} \mathbf{H}$ NMR (400 MHz, CDCl $\left.)_{3}\right): \delta .87(\mathrm{~d}, J=8.0 \mathrm{~Hz}, 1 \mathrm{H}), 7.48(\mathrm{~d}, J=8.4 \mathrm{~Hz}, 2 \mathrm{H})$, 7.26-7.19 (m, 3H), $7.10(\mathrm{~d}, J=8.0 \mathrm{~Hz}, 2 \mathrm{H}), 5.34(\mathrm{~s}, 1 \mathrm{H}), 5.14(\mathrm{~d}, J=15.6 \mathrm{~Hz}, 1 \mathrm{H})$, 
$4.20(\mathrm{~d}, J=15.6 \mathrm{~Hz}, 1 \mathrm{H}), 3.06(\mathrm{t}, J=7.6 \mathrm{~Hz}, 2 \mathrm{H}), 2.31(\mathrm{~s}, 3 \mathrm{H}), 2.02-1.95(\mathrm{~m}, 1 \mathrm{H})$, 1.74-1.67 (m, 1H), 0.79 (s, 9H), 0.77 (s, 3H), 0.32 (s, 9H), -0.09 (s, 3H), -0.10 (s, 3H);

${ }^{13}$ C NMR (100 MHz, $\left.\mathbf{C D C l}_{3}\right): \delta 151.8,143.5,137.2,136.3,136.2,129.1,127.9$, 126.8, 126.7, 126.3, 126.2, 106.5, 106.4, 101.9, 101.3, 59.5, 48.1, 47.2, 41.3, 30.2, 25.9, 21.6, 18.1, 0.0, -5.2, -5.3; IR (neat, $\left.\mathbf{c m}^{-1}\right): v$ 2954, 2928, 2855, 2122, 1597, $1463,1353,1249,1162,1069,833,767,670 ;$ HRMS calculated for $\mathrm{C}_{31} \mathrm{H}_{49} \mathrm{~N}_{2} \mathrm{O}_{3} \mathrm{SSi}_{2}$ $\left(\mathrm{M}+\mathrm{NH}_{4}\right)^{+}:$585.2997; Found: 585.2987.

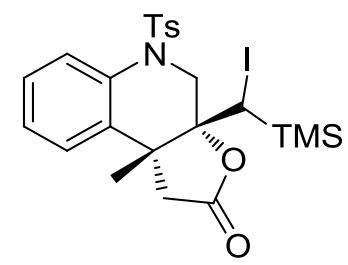

$8 \mathbf{a}$

In a $25 \mathrm{~mL}$ two neck flask, $2 \mathbf{a}\left(0.5 \mathrm{mmol}, 1.0\right.$ equiv.) was dissolved in $\mathrm{CH}_{3} \mathrm{CN}$ (10 $\mathrm{mL}$ ), then NIS ( $2.0 \mathrm{mmol}, 4.0$ equiv.) was added to the mixture. The resulting solution was stirred at room temperature for $10 \mathrm{~h}$. After completion of the reaction, the solvent was evaporated under reduced pressure, the residue was purified by flash column chromatography (petroleum ether : ethyl acetate $=10: 1-5: 1$ ) to obtain the compound 8a.

White solid; m.p.: $196-198{ }^{\circ} \mathrm{C}$; $(222 \mathrm{mg} ; 78 \%$ yield; $91 \%$ ee $)$; [The ee value was determined by chiral HPLC using a Chiralpak IC-3 column with hexane : isopropanol $=70: 30$ at a flow rate $0.7 \mathrm{~mL} / \mathrm{min}$ detected at $214 \mathrm{~nm}$ wavelength. Elution time: $\mathrm{t}($ minor $)=14.3 \mathrm{~min} ; \mathrm{t}($ major $)=19.6 \mathrm{~min} ;[\alpha]_{\mathrm{D}}{ }^{29}=26.76\left(\mathrm{c}=1.0\right.$, in $\left.\left.\mathrm{CDCl}_{3}\right)\right] .{ }^{1} \mathbf{H}$ NMR (400 MHz, CDCl $): \delta 7.79(\mathrm{~d}, J=8.4 \mathrm{~Hz}, 2 \mathrm{H}), 7.60(\mathrm{dd}, J=8.4 \mathrm{~Hz}, 0.8 \mathrm{~Hz}$, 1H), 7.32-7.30 (m, 3H), $7.22(\mathrm{t}, J=7.6 \mathrm{~Hz}, 1 \mathrm{H}), 7.13(\mathrm{t}, J=7.6 \mathrm{~Hz}, 1 \mathrm{H}), 4.62$ (d, $J=$ 14.0 Hz, 1H), 3.92 (d, $J=14.4 \mathrm{~Hz}, 1 \mathrm{H}), 3.32(\mathrm{~s}, 1 \mathrm{H}), 2.83$ (d, $J=17.6 \mathrm{~Hz}, 1 \mathrm{H}), 2.49$ $(\mathrm{d}, J=17.6 \mathrm{~Hz}, 1 \mathrm{H}), 2.41(\mathrm{~s}, 3 \mathrm{H}), 1.81(\mathrm{~s}, 3 \mathrm{H}), 0.33$ (s, 9H); ${ }^{\mathbf{1 3}} \mathbf{C}$ NMR (100 MHz, $\left.\mathbf{C D C l}_{3}\right): \delta 172.5,144.3,136.9,136.6,134.5,130.0,127.9,127.5,126.9,125.4,121.5$, 91.7, 53.8, 47.1, 45.5, 22.4, 21.7, 17.6, 0.83; IR (neat, $\mathbf{~ c m}^{-1}$ ): $v 2959,2923,1780$, 1596, 1348, 1254, 1160, 1080, 1025, 991, 844, 804, 665; HRMS calculated for $\mathrm{C}_{23} \mathrm{H}_{32} \mathrm{IN}_{2} \mathrm{O}_{4} \mathrm{SSi}\left(\mathrm{M}+\mathrm{NH}_{4}\right)^{+}:$587.0891; Found: 587.0879 . 


\section{Deuterium labeling studies}

The procedures for deuterium-labeling experiments were similar to the reaction as shown in Scheme 2 by using $\mathrm{CH}_{3} \mathrm{CH}_{2} \mathrm{OD}$ and $\mathrm{CH}_{3} \mathrm{CD}_{2} \mathrm{OH}$ as the solvents.

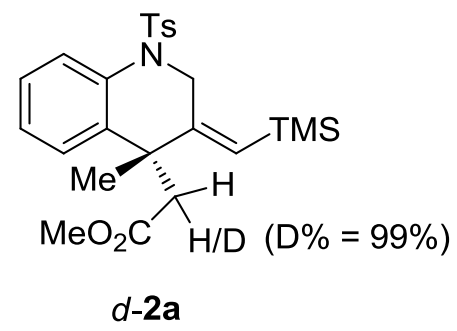

${ }^{1}$ H NMR (400 MHz, CDCl 3$): \delta 7.79(\mathrm{~d}, J=8.0 \mathrm{~Hz}, 1 \mathrm{H}), 7.42(\mathrm{~d}, J=8.0 \mathrm{~Hz}, 2 \mathrm{H})$, 7.23-7.20 (m, 1H), 7.16-7.15 (m, 2H), $7.11(\mathrm{~d}, J=8.0 \mathrm{~Hz}, 2 \mathrm{H}), 5.46(\mathrm{~s}, 1 \mathrm{H}), 4.85(\mathrm{~d}$, $J=15.6 \mathrm{~Hz}, 1 \mathrm{H}), 4.27(\mathrm{~d}, J=15.2 \mathrm{~Hz}, 1 \mathrm{H}), 3.46(\mathrm{~s}, 3 \mathrm{H}), 2.75(\mathrm{~s}, 1 \mathrm{H}), 2.32(\mathrm{~s}, 3 \mathrm{H})$, $0.75(\mathrm{~s}, 3 \mathrm{H}), 0.25(\mathrm{~s}, 9 \mathrm{H})$.

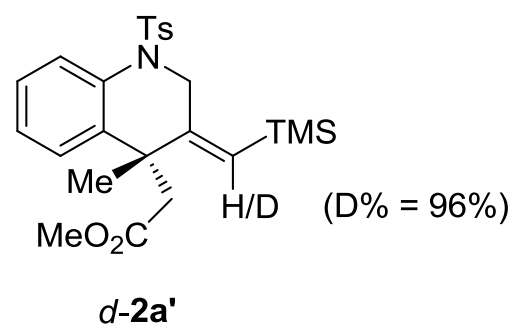

${ }^{1}$ H NMR (400 MHz, $\left.\mathbf{C D C l}_{3}\right): \delta 7.79(\mathrm{~d}, J=7.6 \mathrm{~Hz}, 1 \mathrm{H}), 7.42(\mathrm{~d}, J=8.4 \mathrm{~Hz}, 2 \mathrm{H})$, 7.23-7.20 (m, 1H), 7.16-7.15 (m, 2H), $7.11(\mathrm{~d}, J=8.4 \mathrm{~Hz}, 2 \mathrm{H}), 5.46(\mathrm{~s}, 0.04 \mathrm{H}), 4.85$ $(\mathrm{d}, J=15.2 \mathrm{~Hz}, 1 \mathrm{H}), 4.27(\mathrm{~d}, J=15.6 \mathrm{~Hz}, 1 \mathrm{H}), 3.46(\mathrm{~s}, 3 \mathrm{H}), 2.77(\mathrm{~d}, J=15.6 \mathrm{~Hz}, 1 \mathrm{H})$, $2.50(\mathrm{~d}, J=15.2 \mathrm{~Hz}, 1 \mathrm{H}), 2.32(\mathrm{~s}, 3 \mathrm{H}), 0.76(\mathrm{~s}, 3 \mathrm{H}), 0.25(\mathrm{~s}, 9 \mathrm{H})$. 
9. Copies of ${ }^{1} \mathrm{H}$ NMR, ${ }^{13} \mathrm{C}$ NMR and ${ }^{19}$ F NMR spectra<smiles>[3H]N(CC#CC(C)(C)C)c1ccccc1C(C)=CC(=O)OC</smiles>

$1 a$
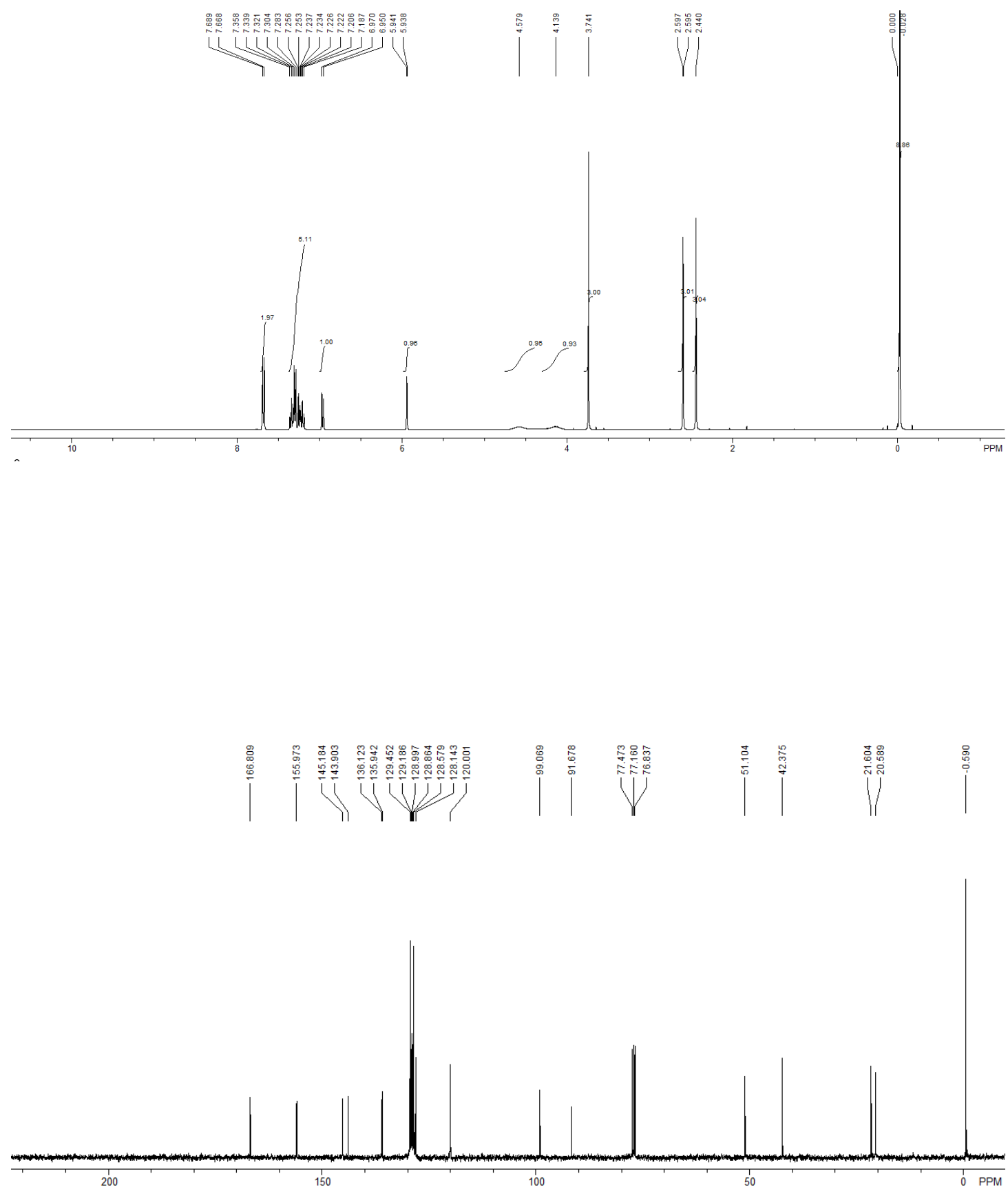
<smiles>CC(=O)CC=C(C)c1cc(C)ccc1N([135I])CC#CC(C)(C)C</smiles>

1b

ソิن

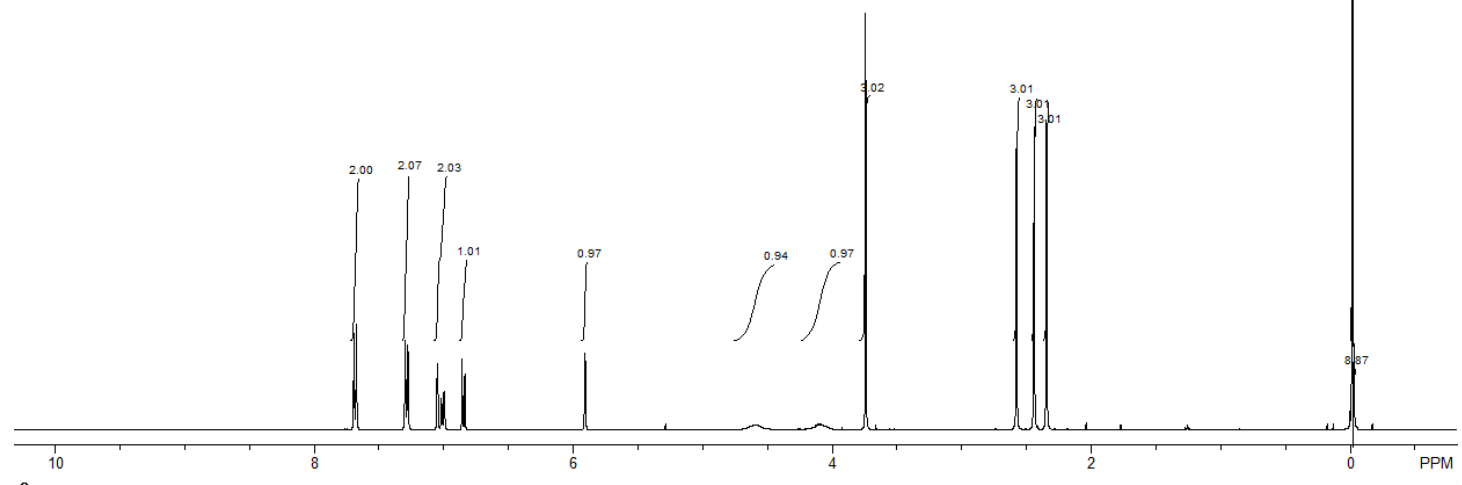

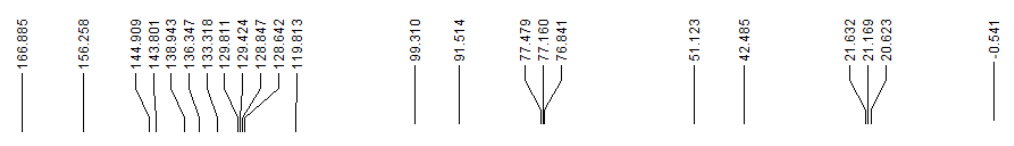

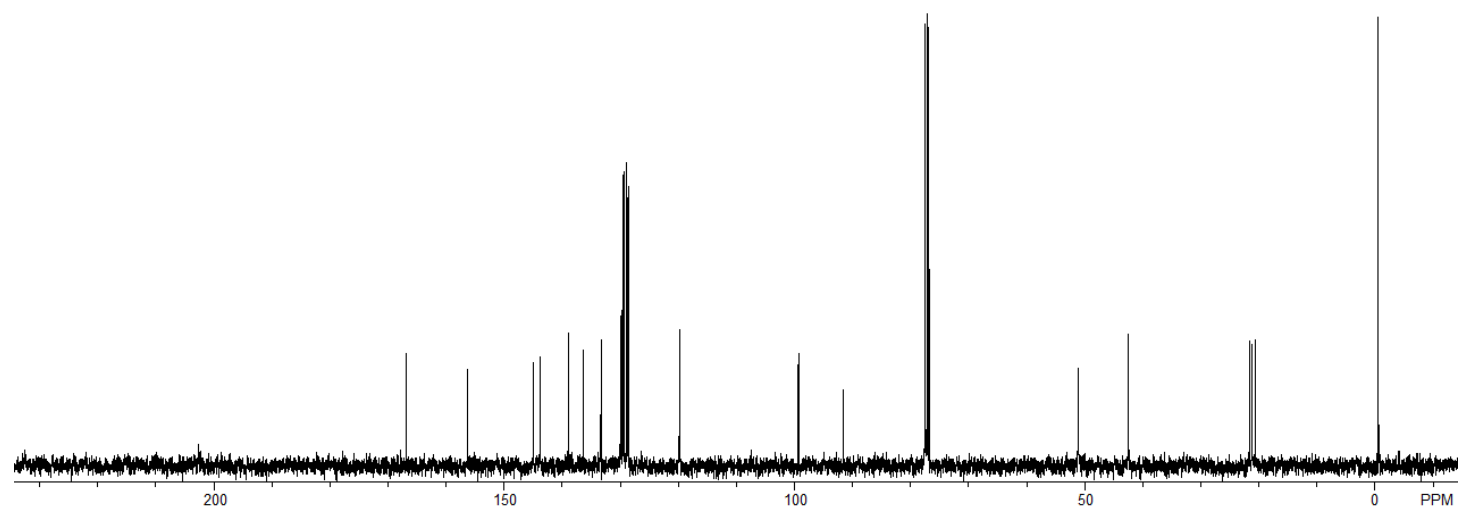



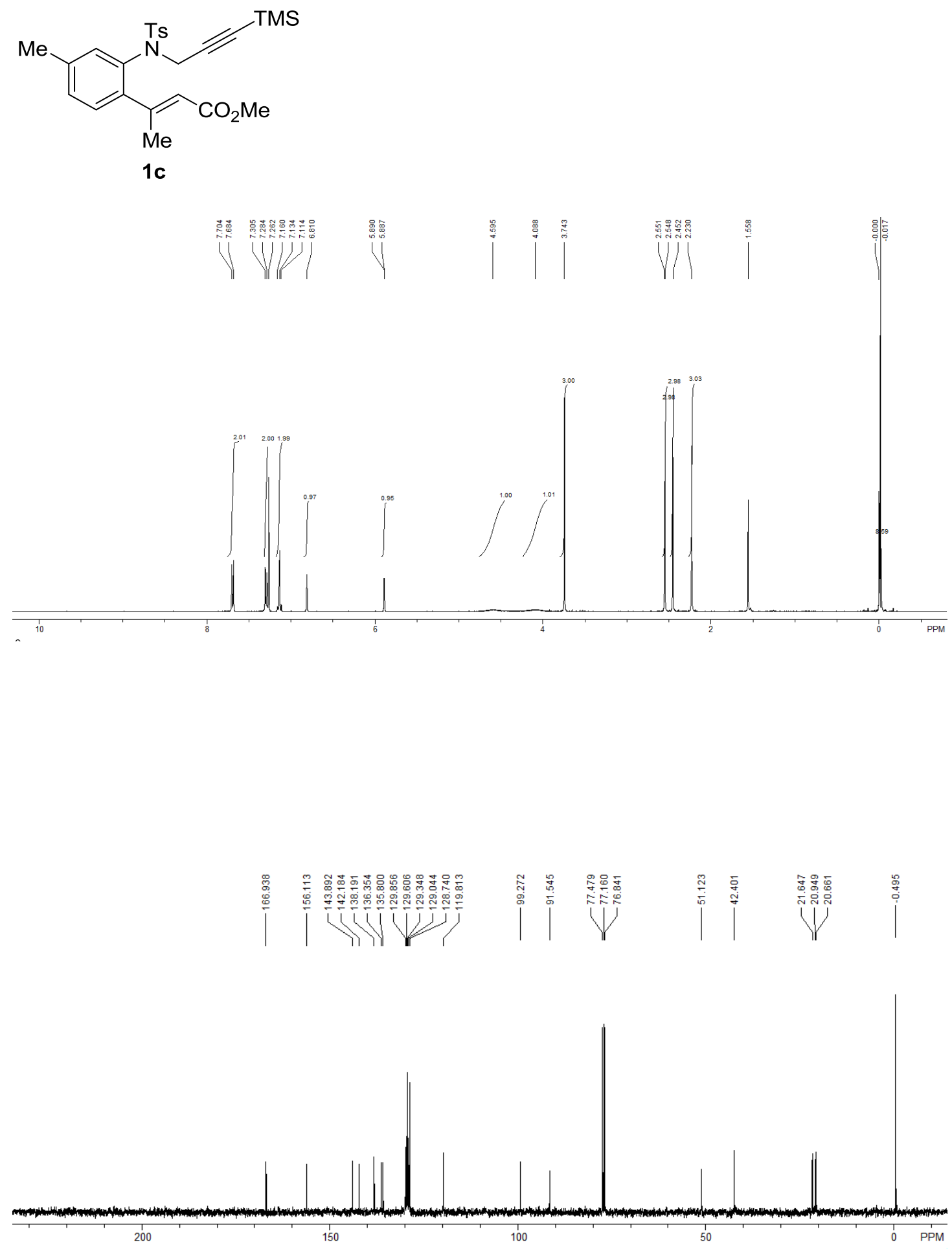

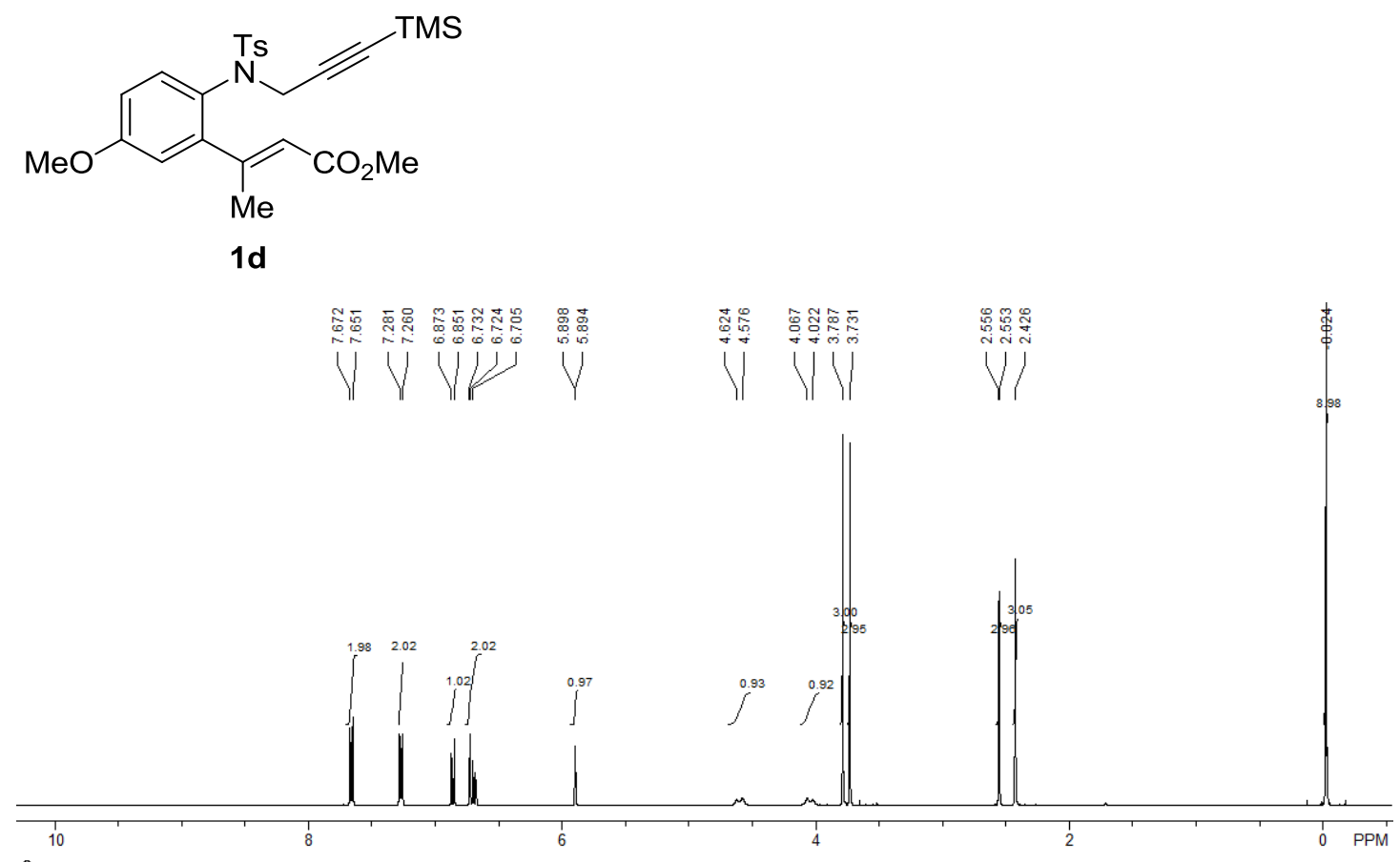

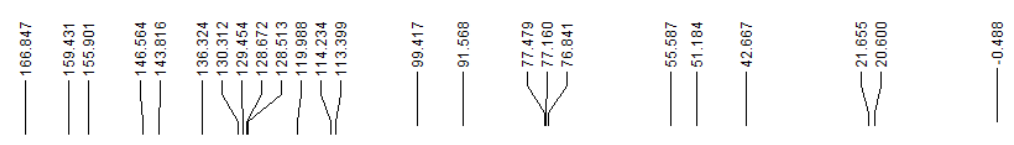

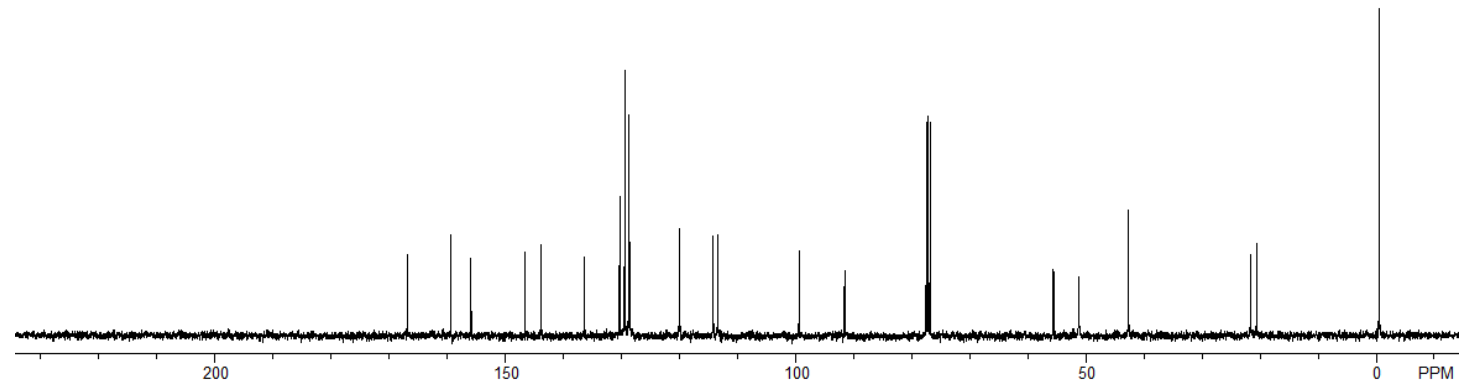



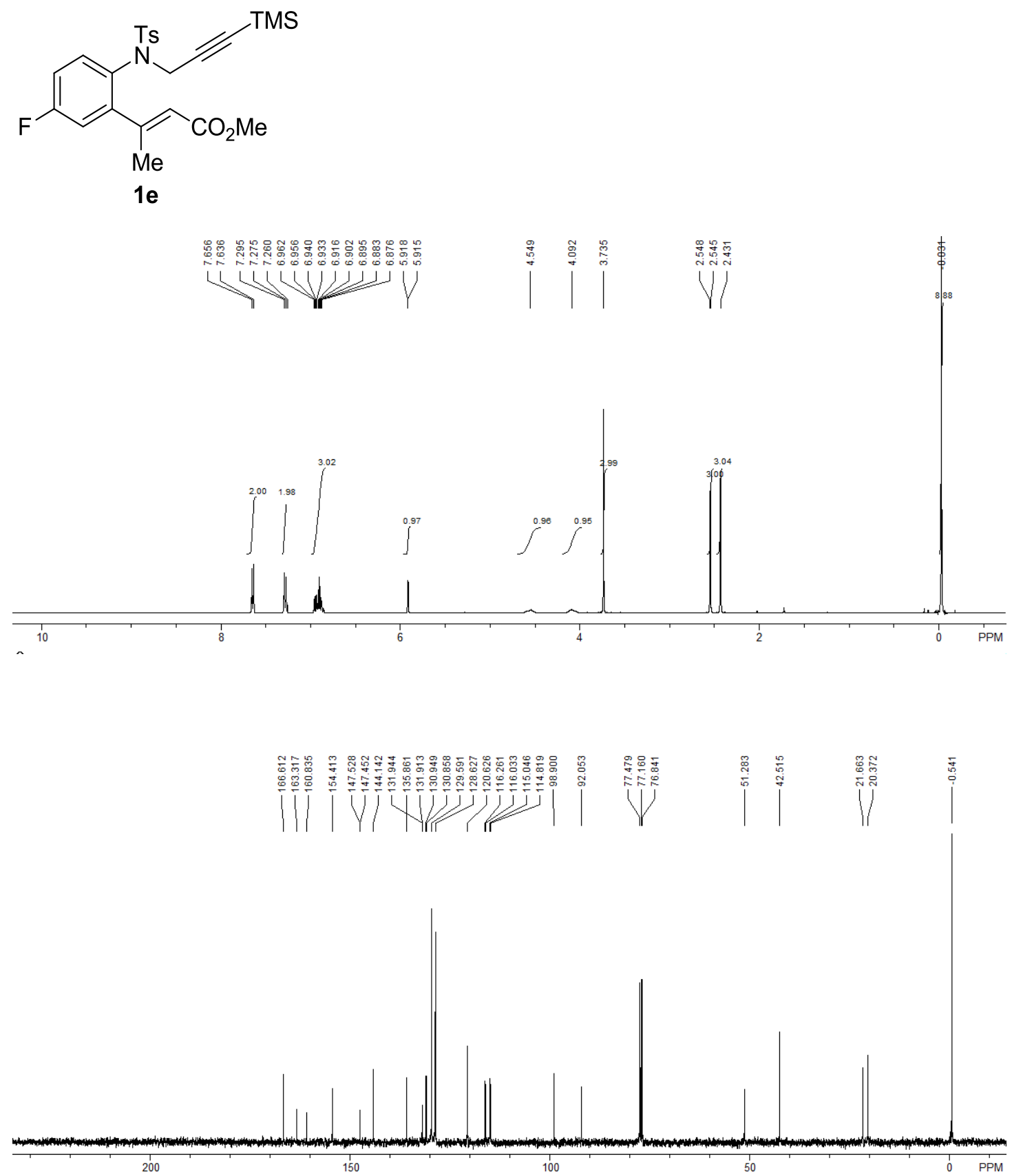


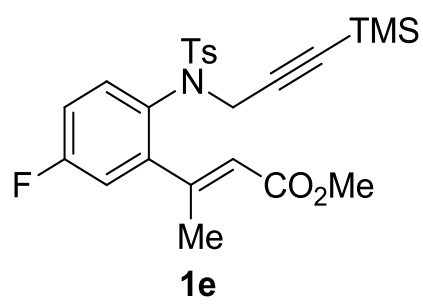

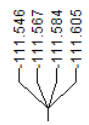

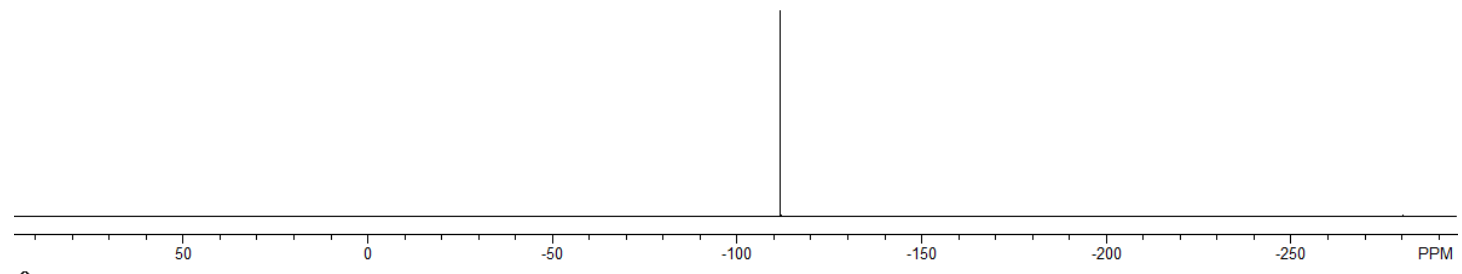



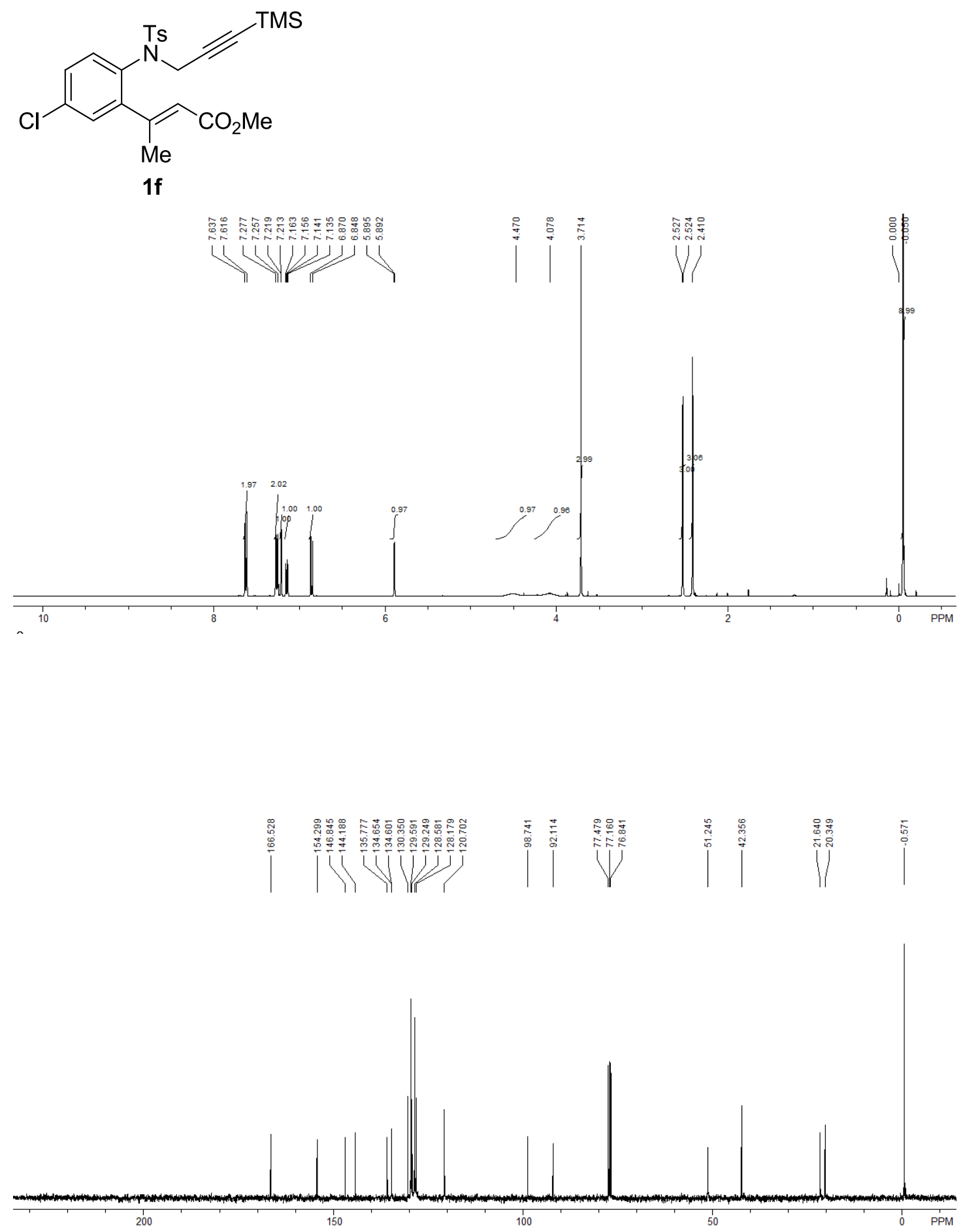
<smiles></smiles>

$1 \mathrm{~g}$
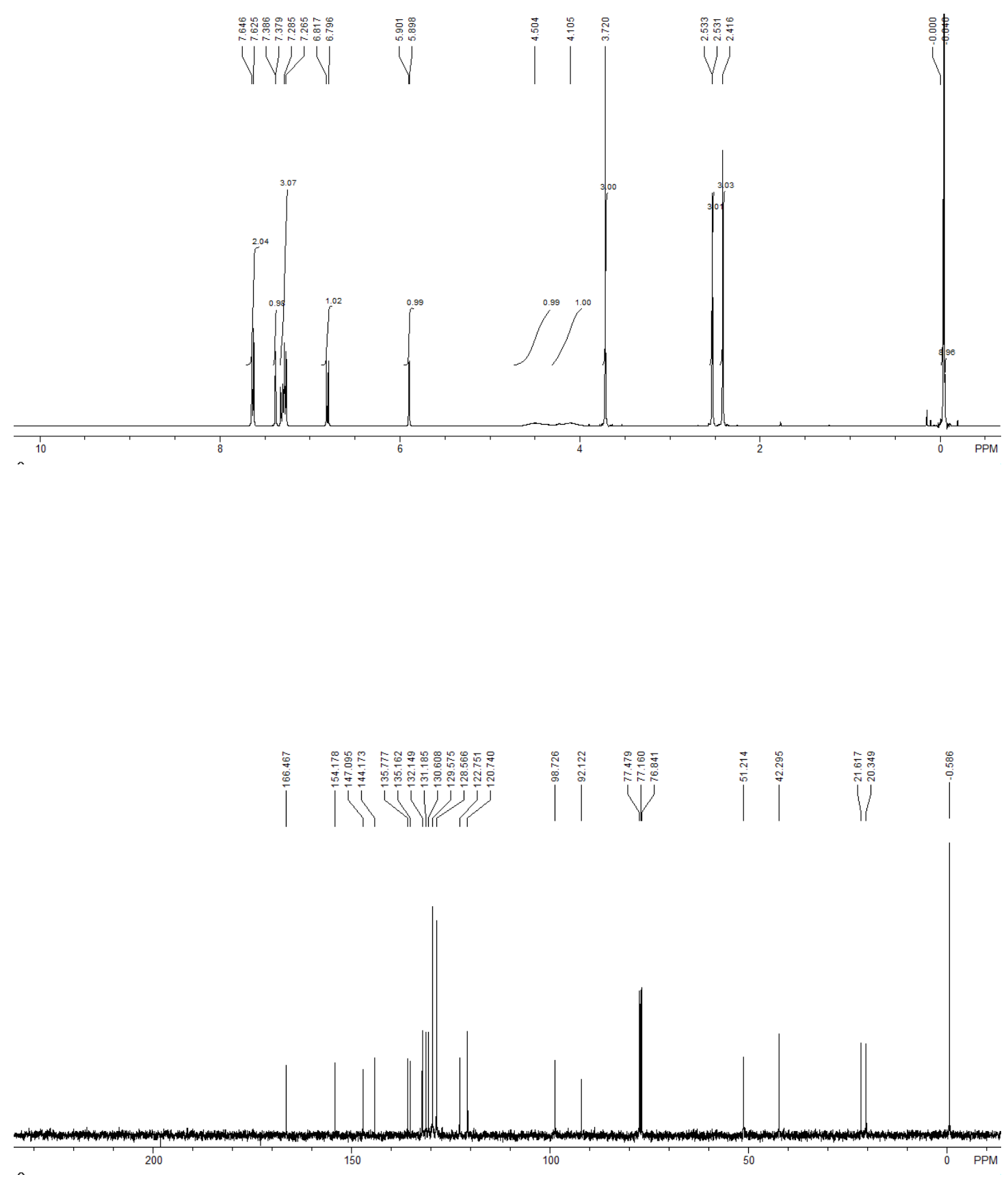
<smiles>[3H]N(CC#CC(C)=O)c1ccc(C(F)(F)F)cc1C(C)=CCC(C)=O</smiles>

1h
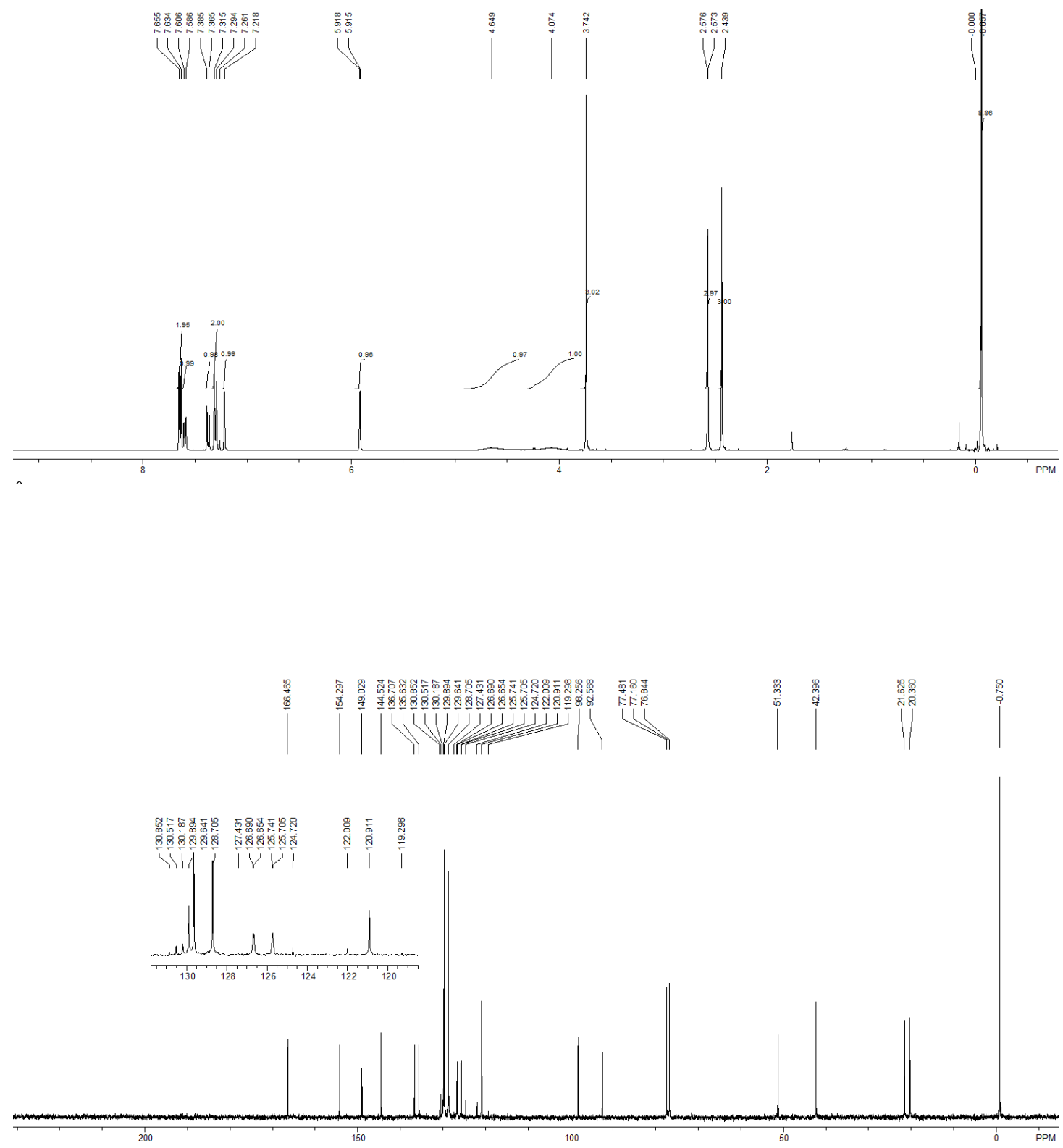


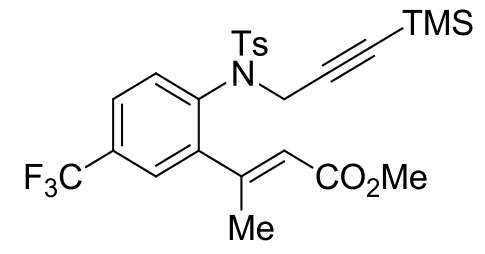

1h

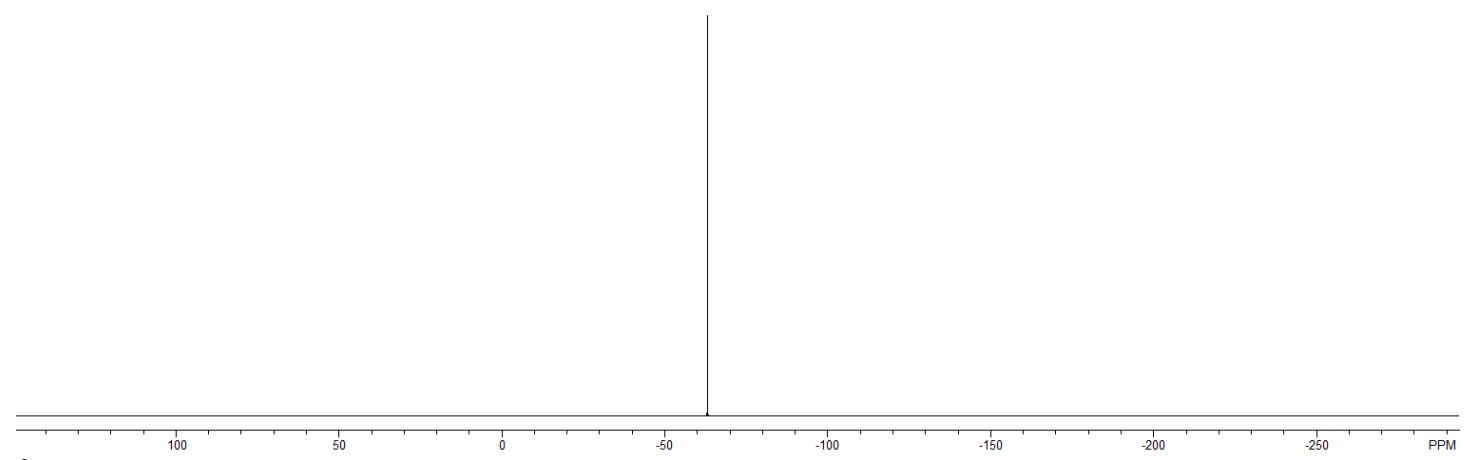




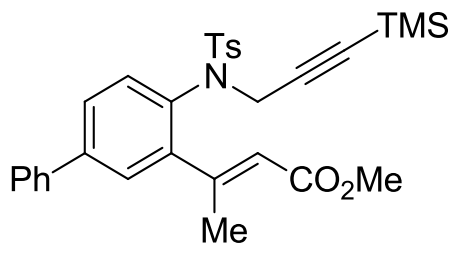

$1 \mathrm{i}$
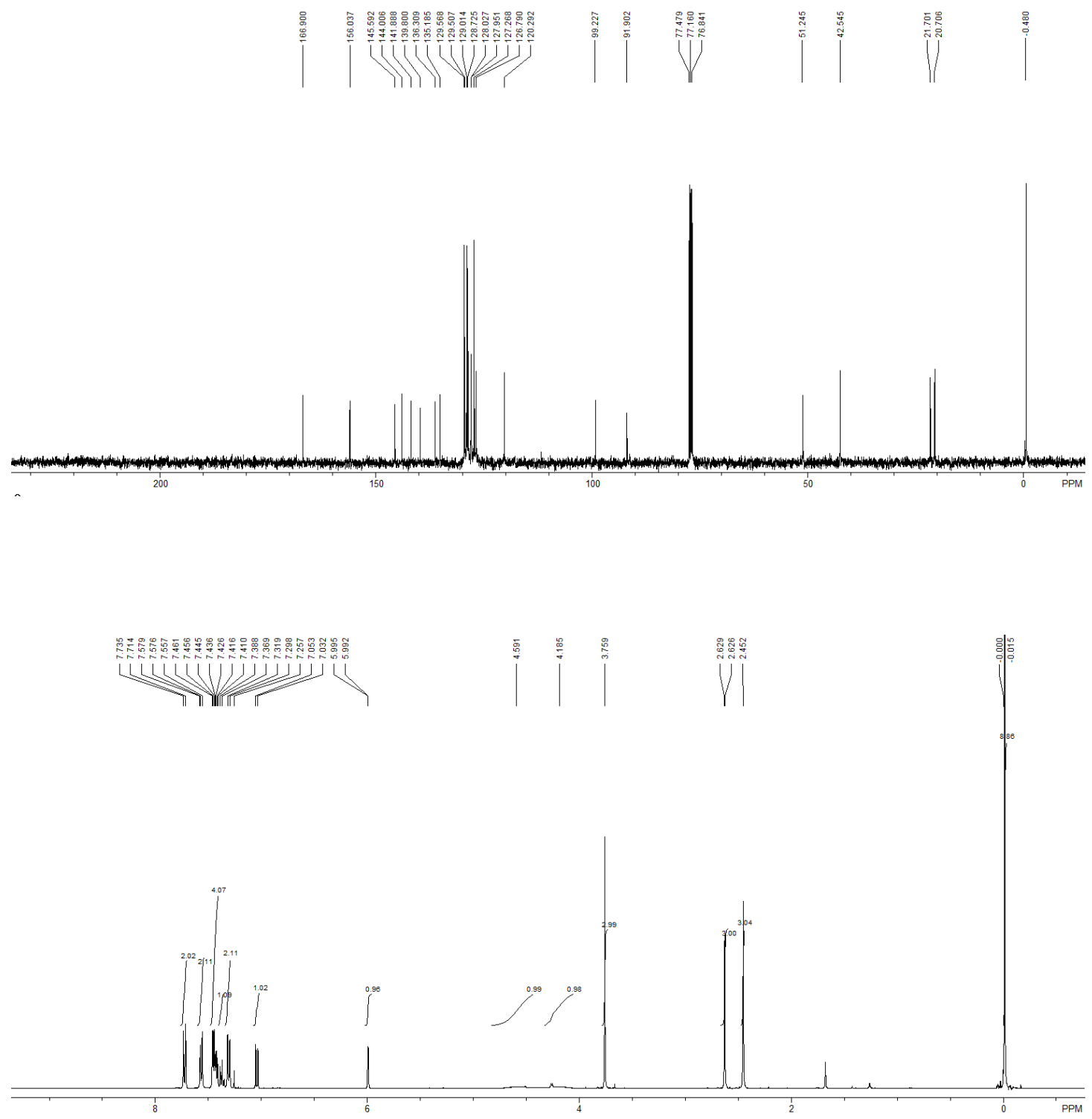


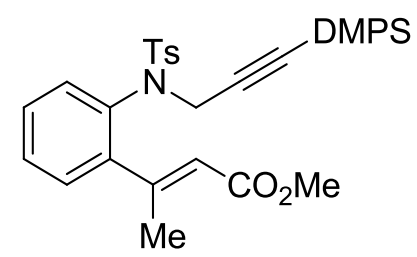

1j
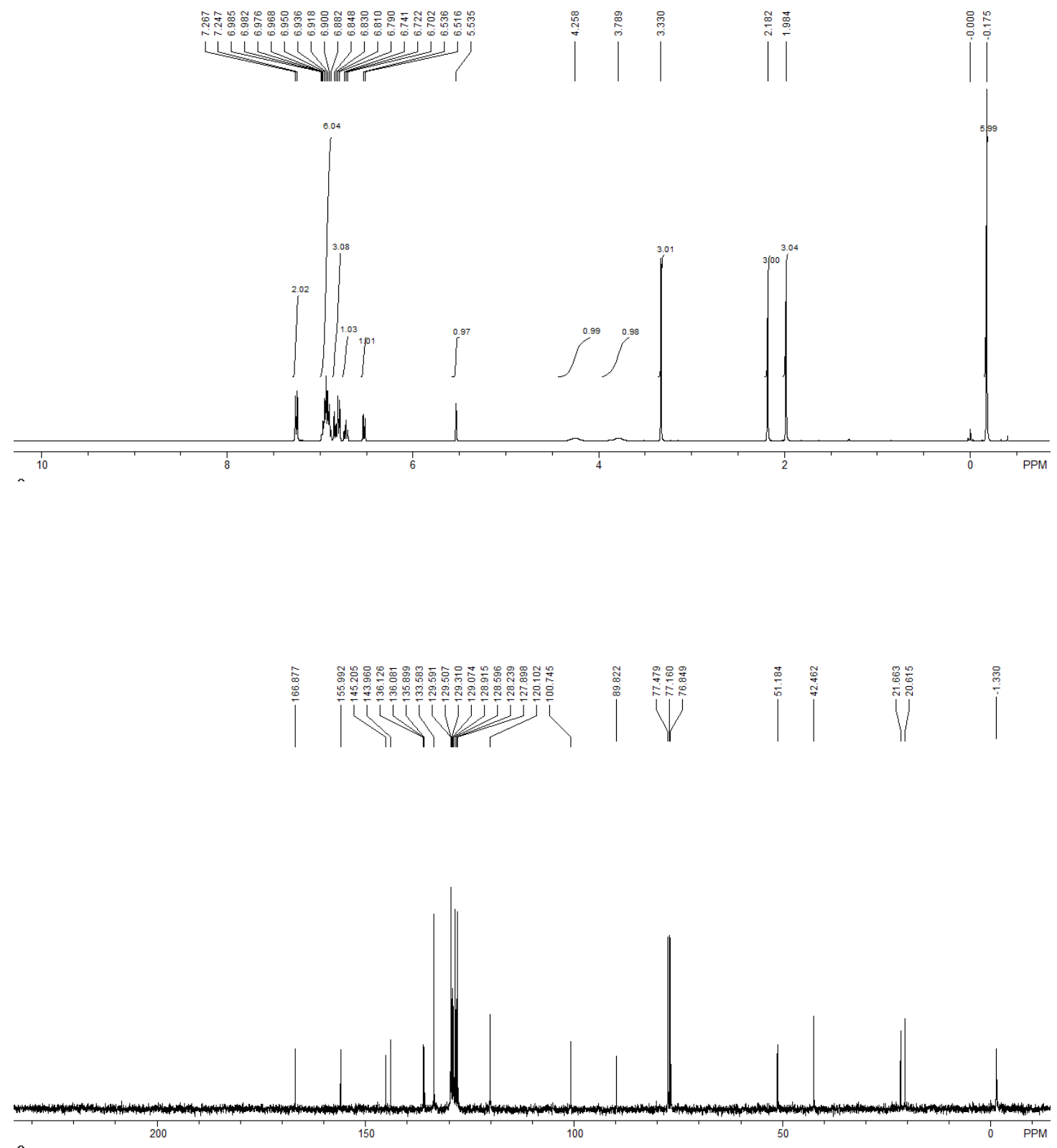

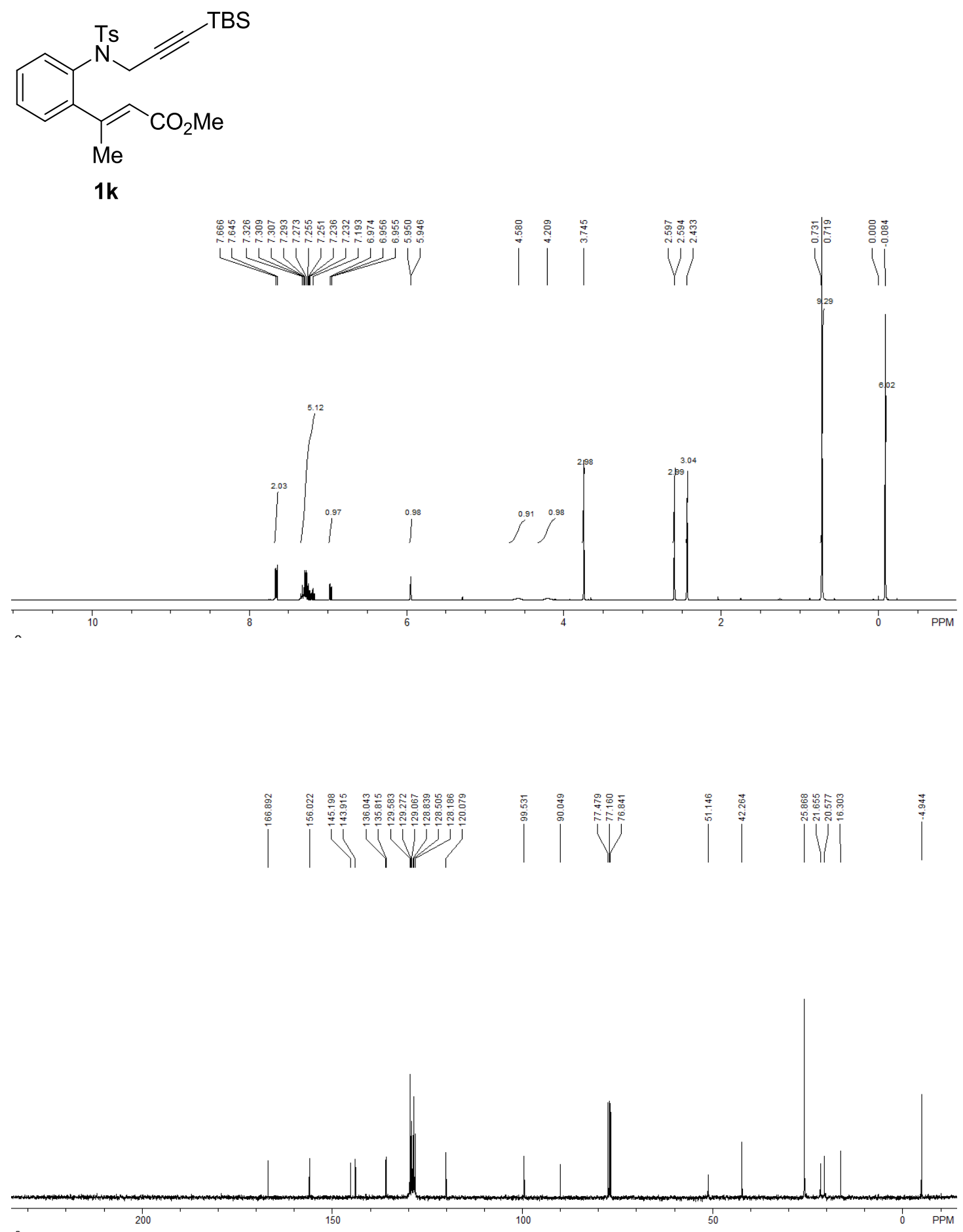


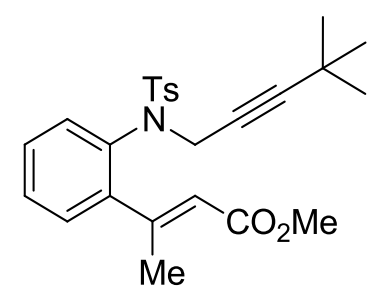

1 I

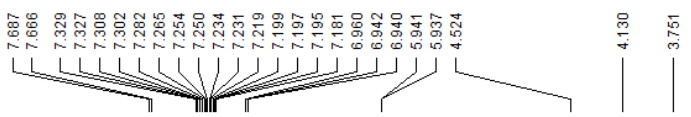

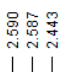

Vid

i

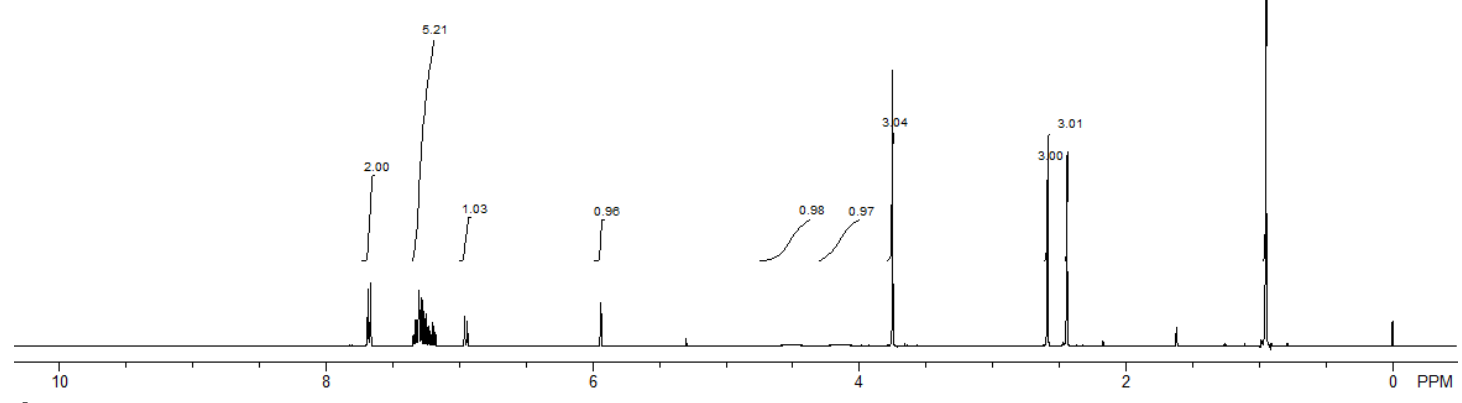

$\left.\right|^{\infty}$

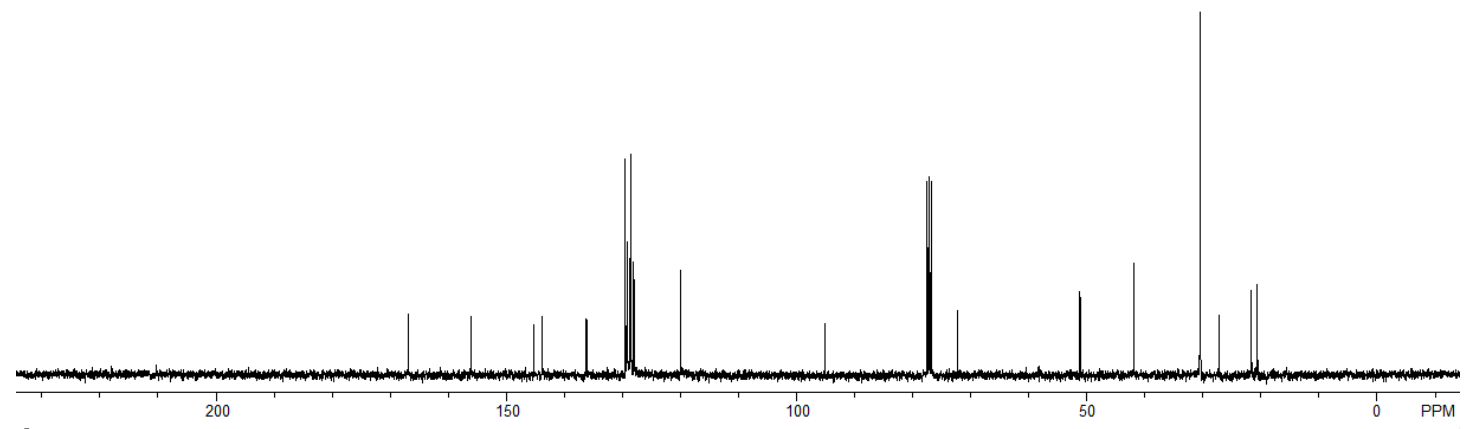



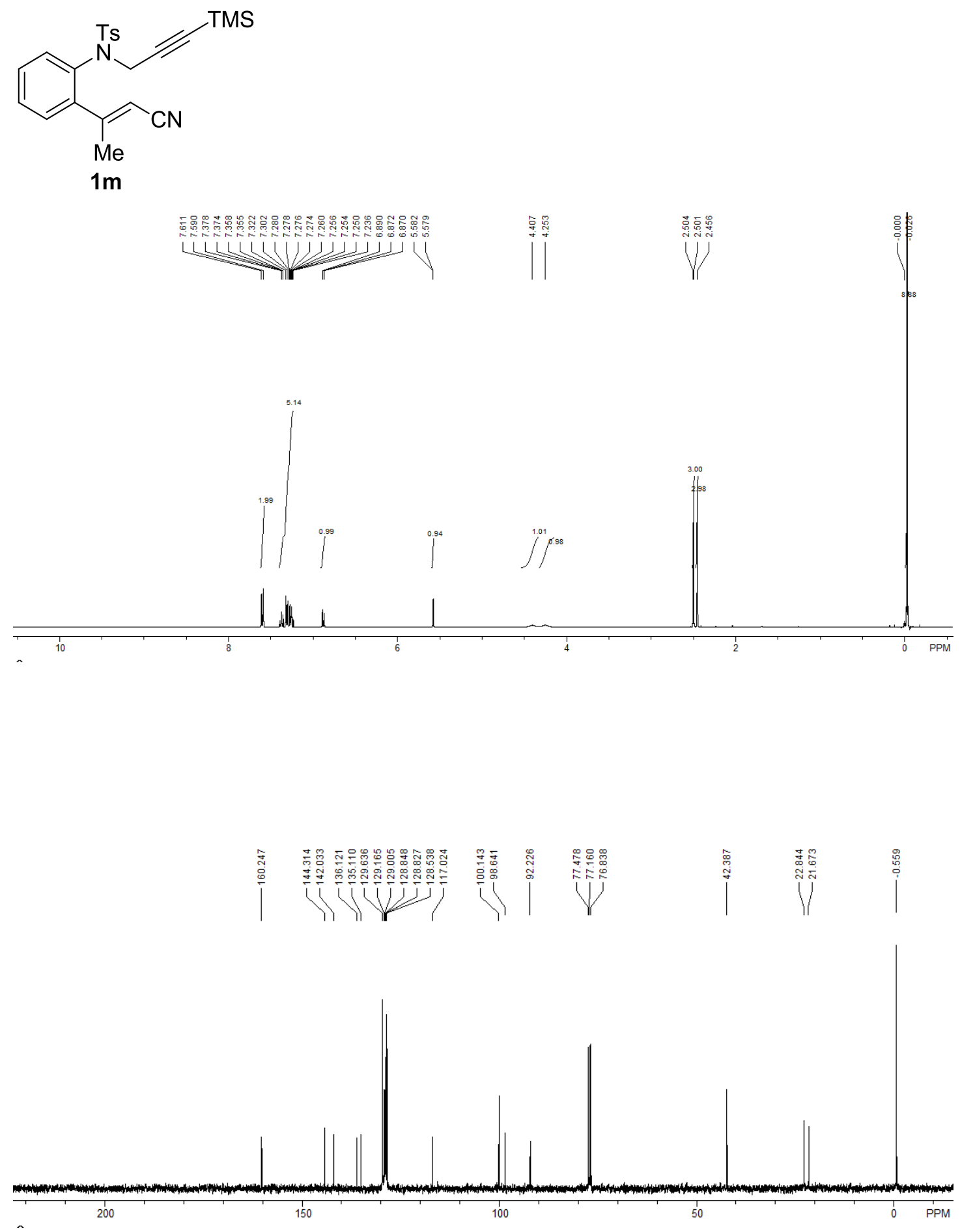

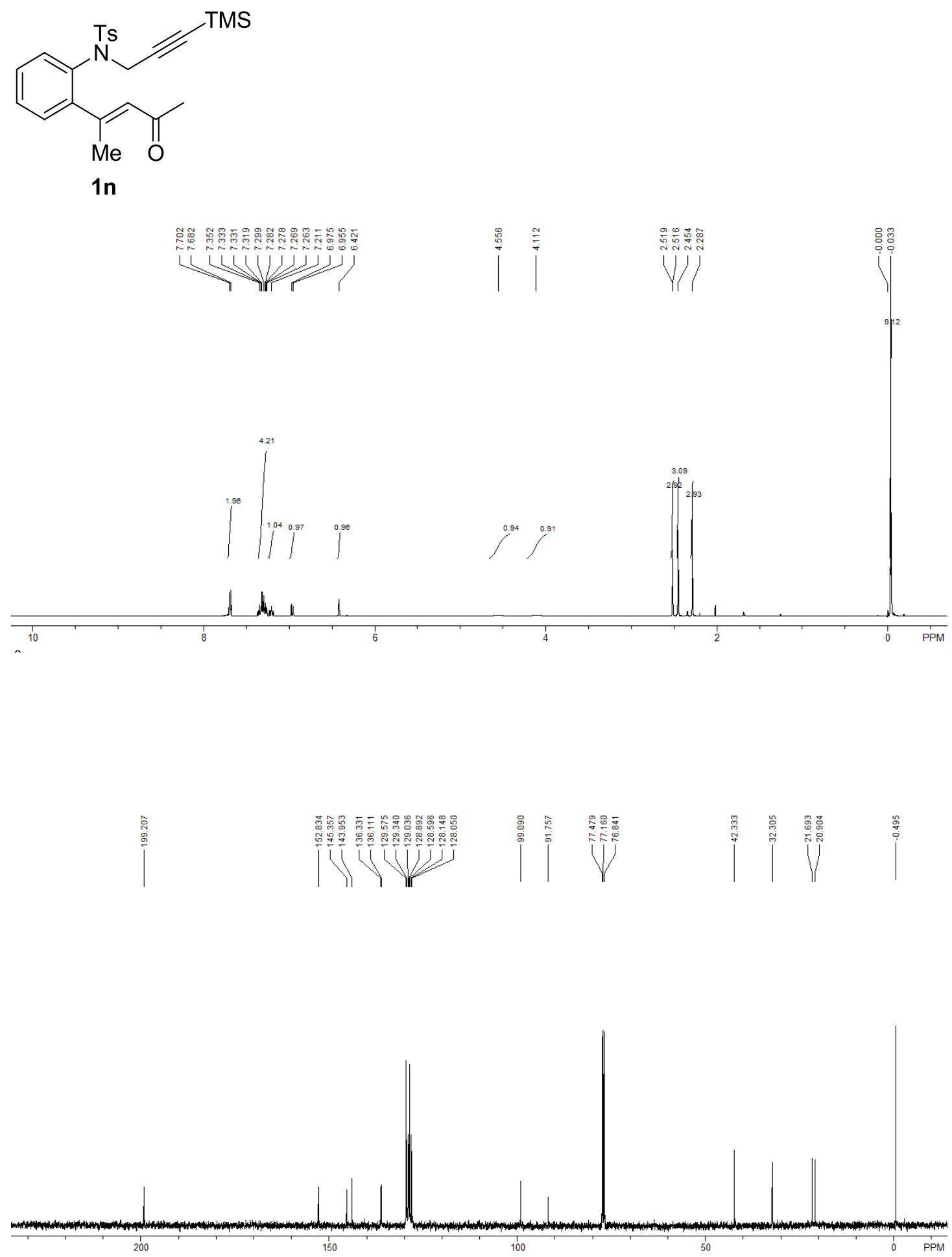

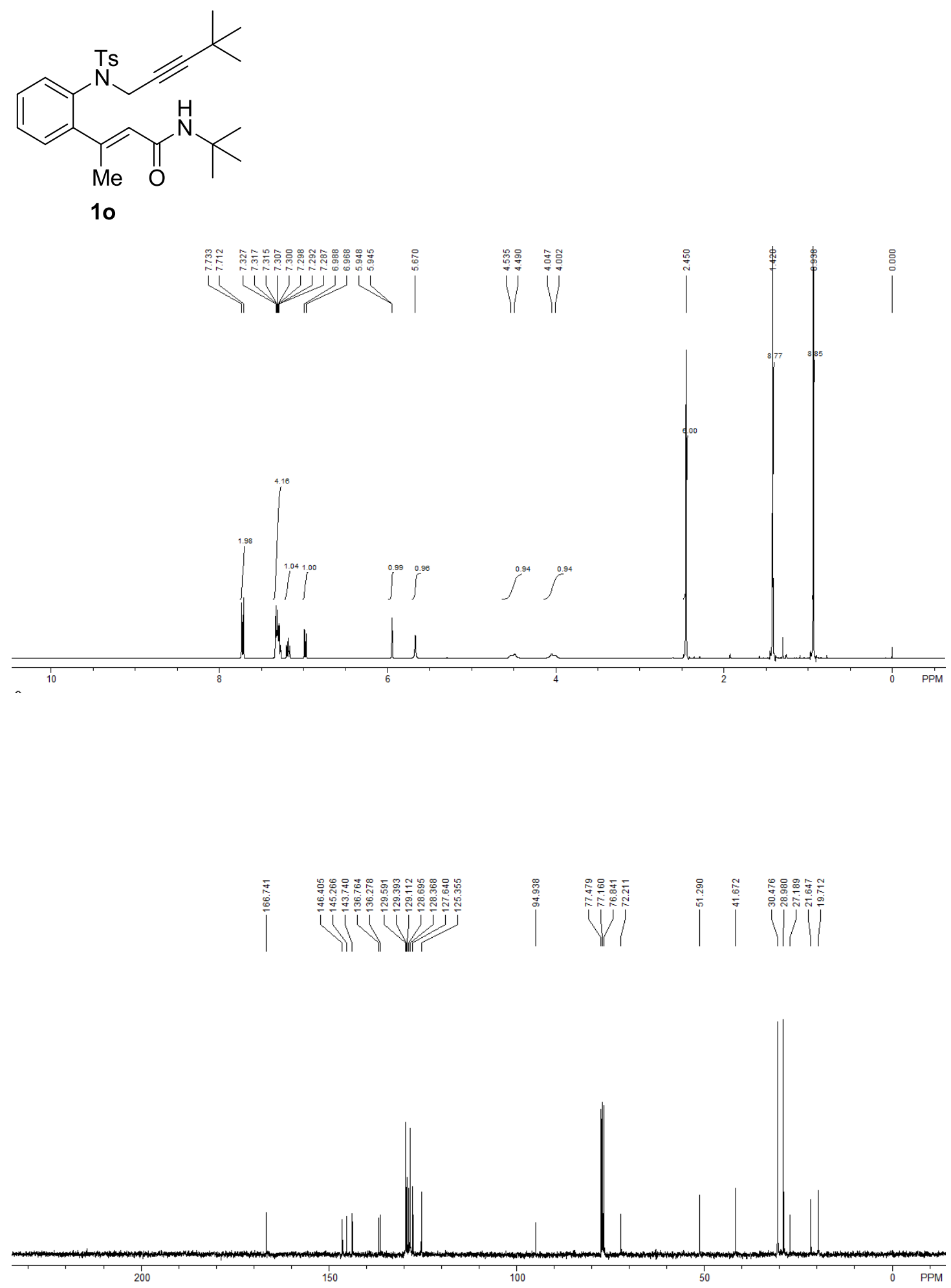


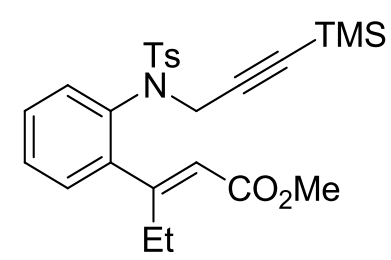

$1 \mathrm{p}$

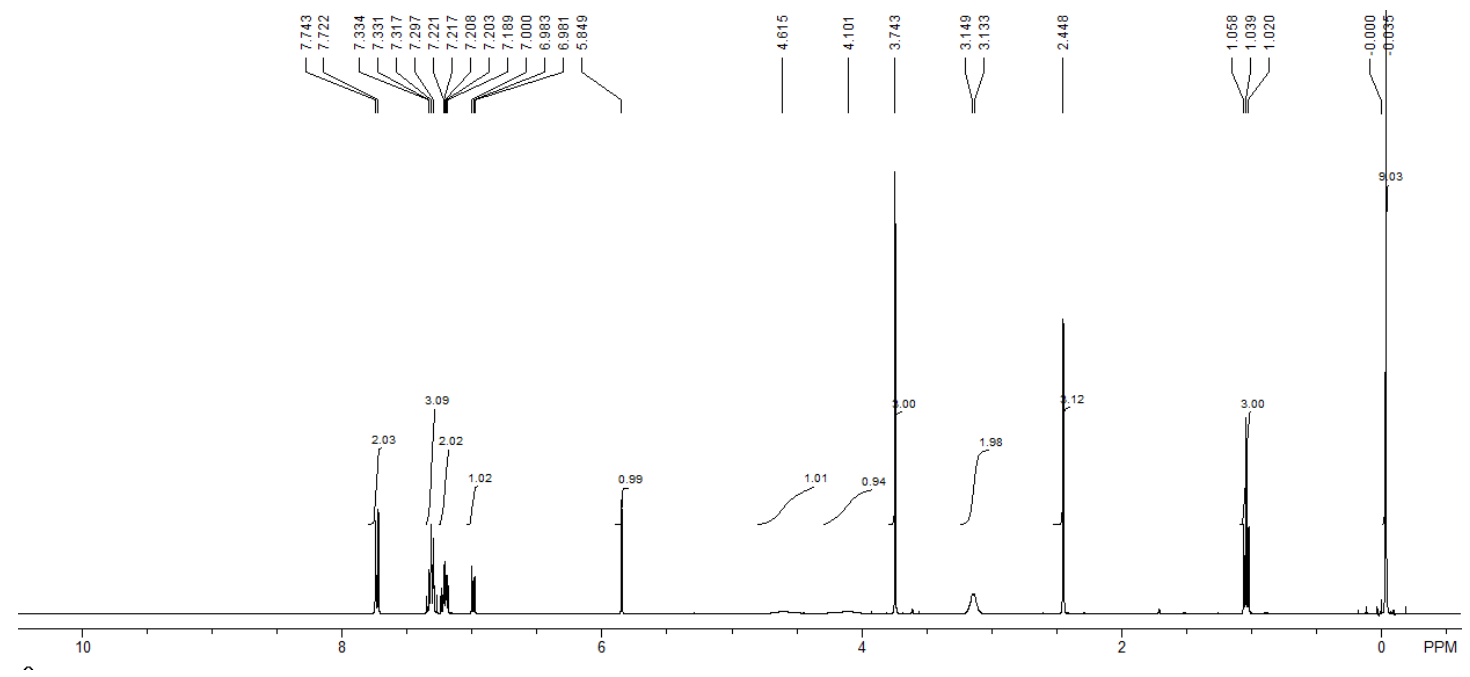

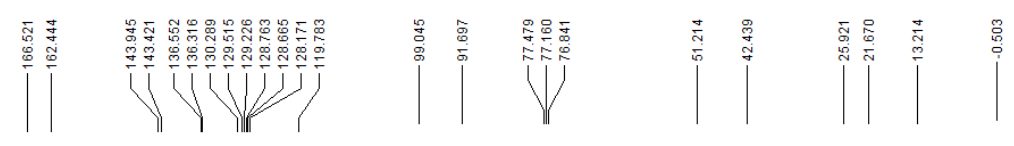

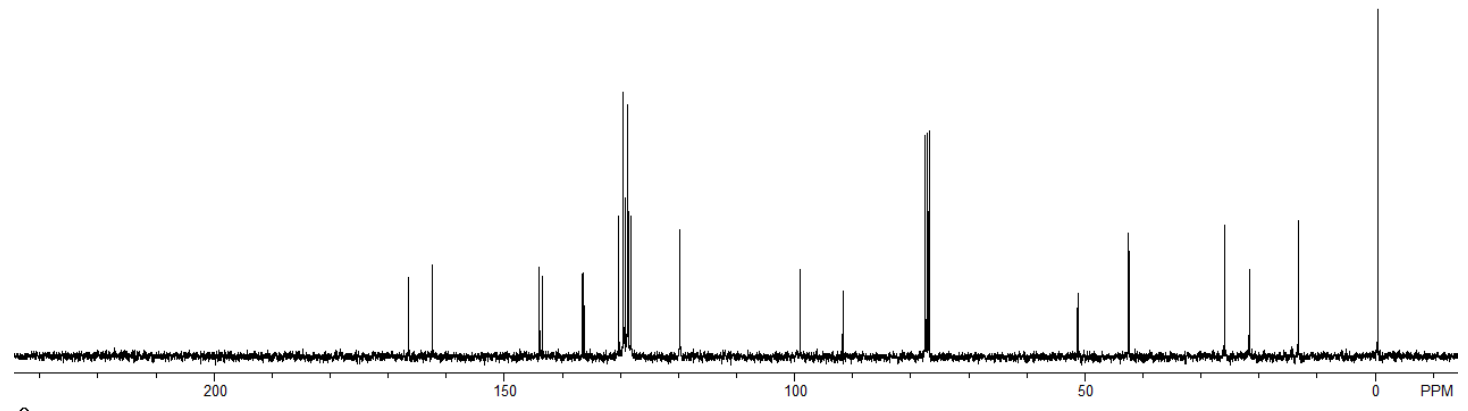



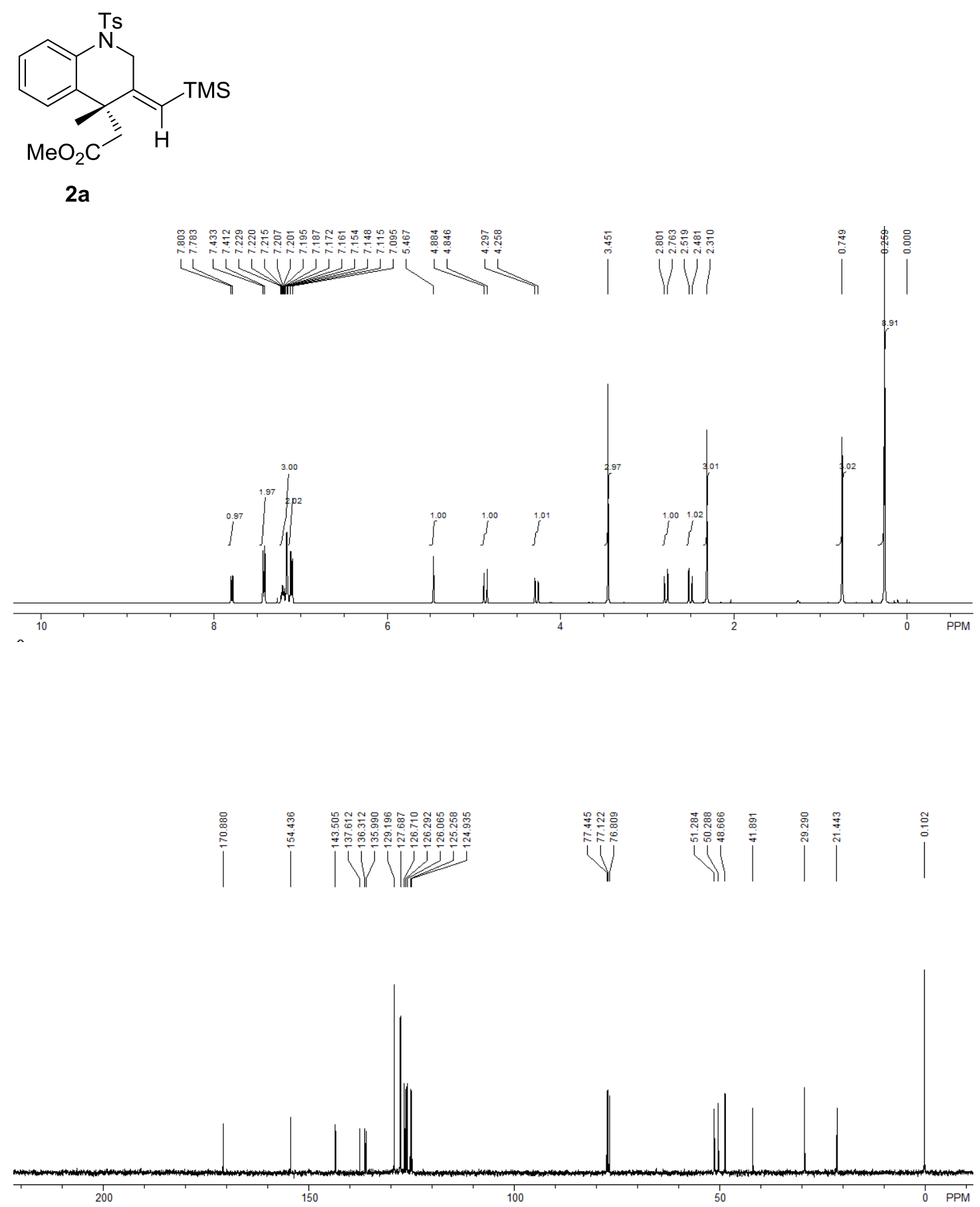
<smiles>CC[C@]1(C)/C(=C/C(C)=O)CN([As])c2ccc(C)cc21</smiles>
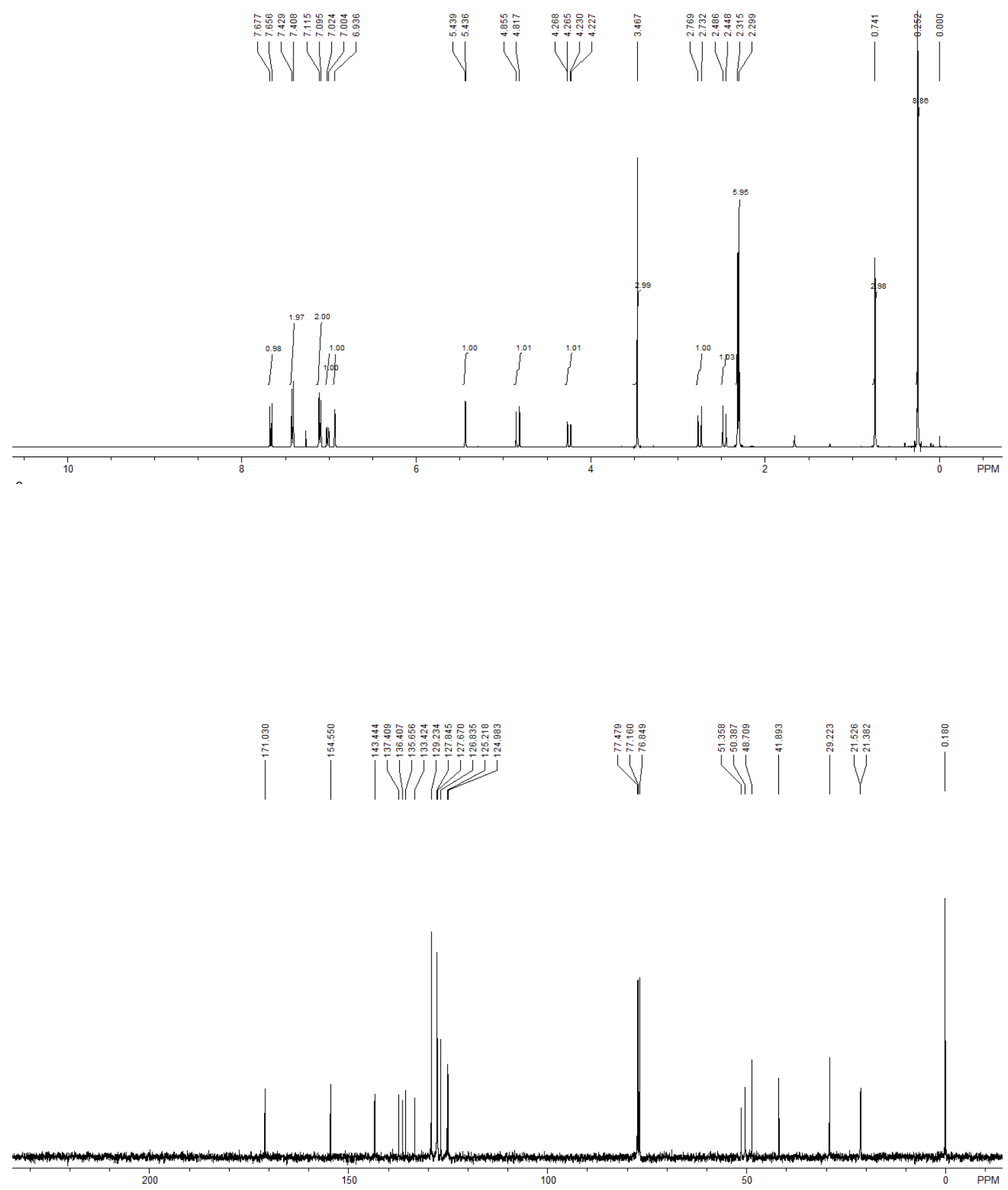

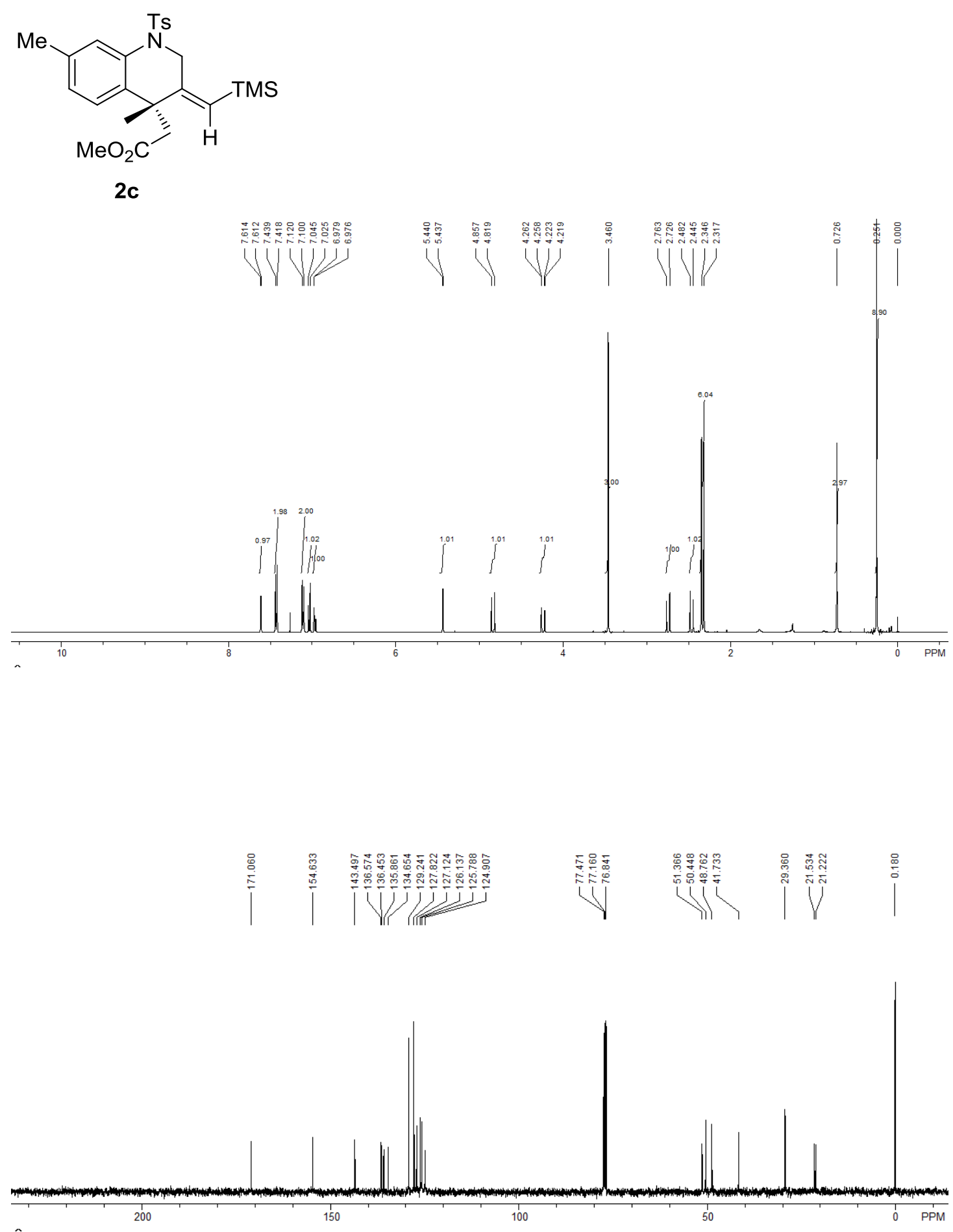
<smiles>CC[C@]1(C)/C(=C\C(C)=S)CN([As])c2ccc(OC)cc21</smiles>

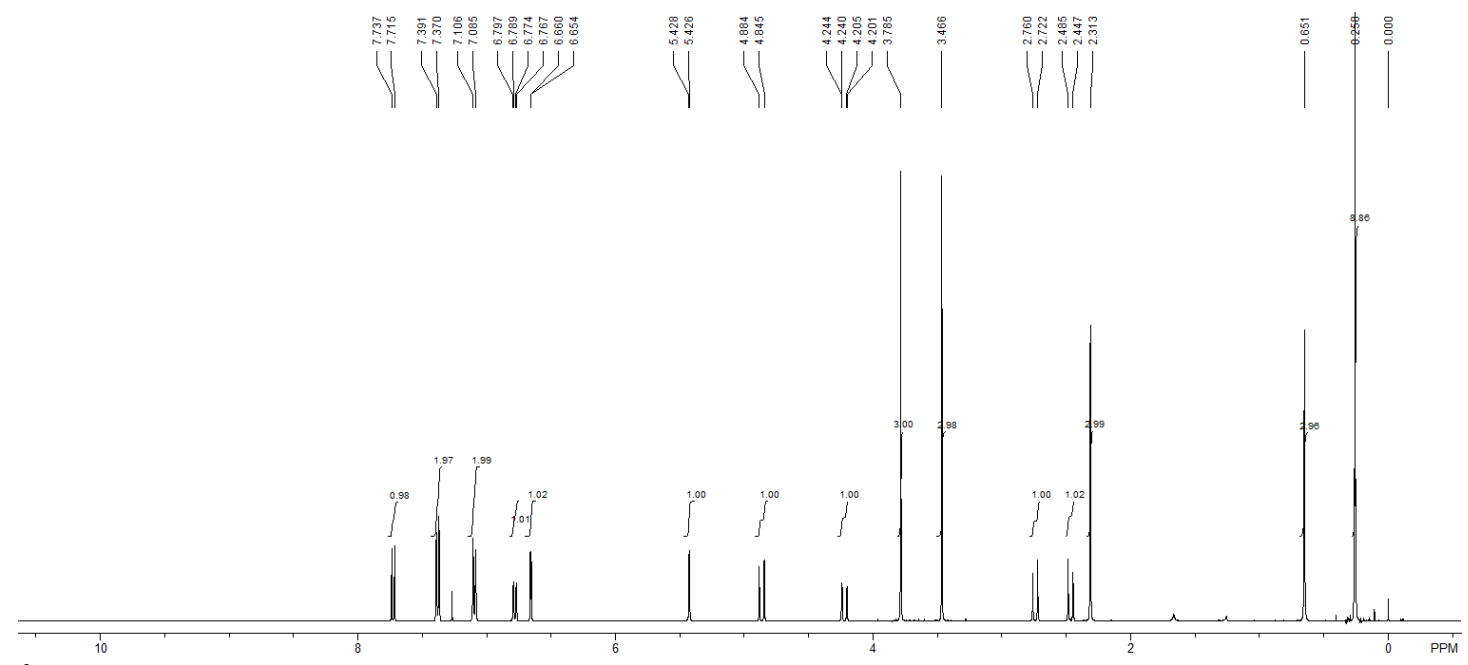

$\left.\right|^{1}$

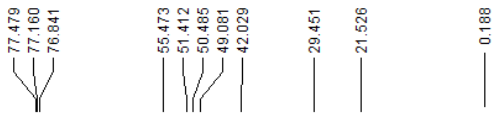

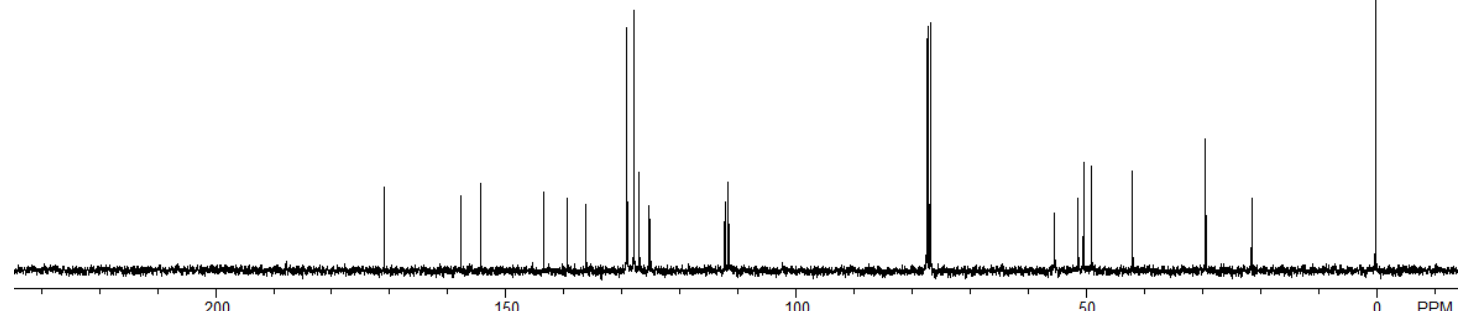


<smiles>CC(=O)C[C@@]1(C)C(=C(C)S(C)(=O)=O)C[N-]c2ccc(F)cc21</smiles>

$2 e$
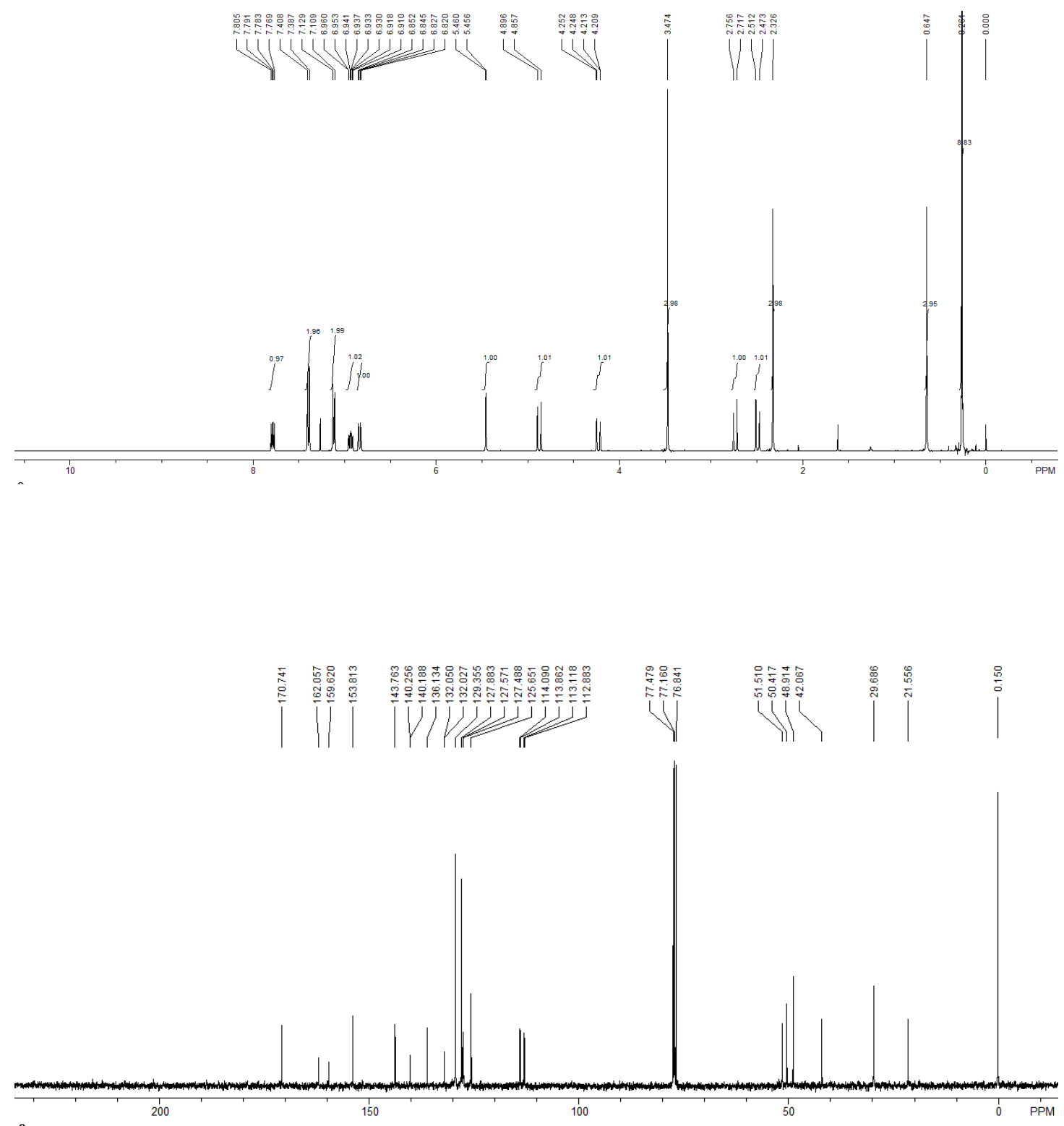


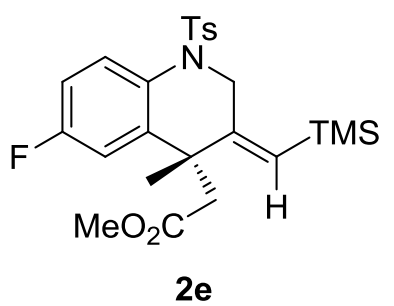

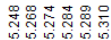

닌

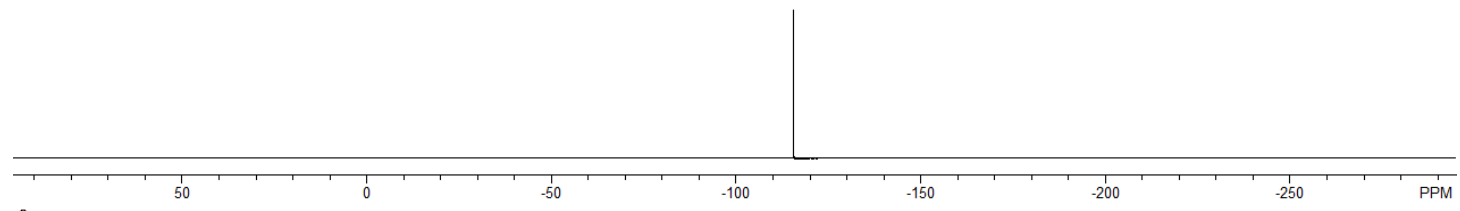


<smiles>CC(=O)CC1(CC(C)=O)/C(=C/[Y5](C)(C)C)CN([As])c2ccc(Cl)cc21</smiles>

$2 f$

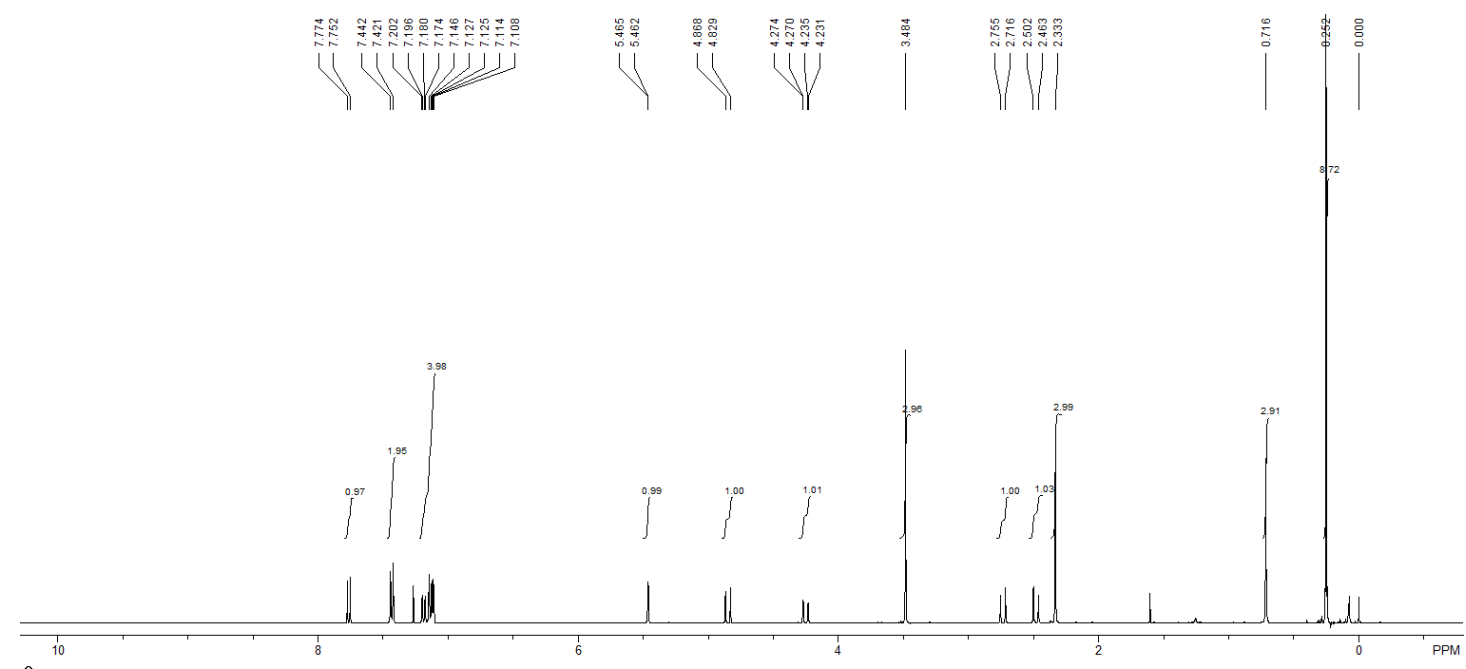

|

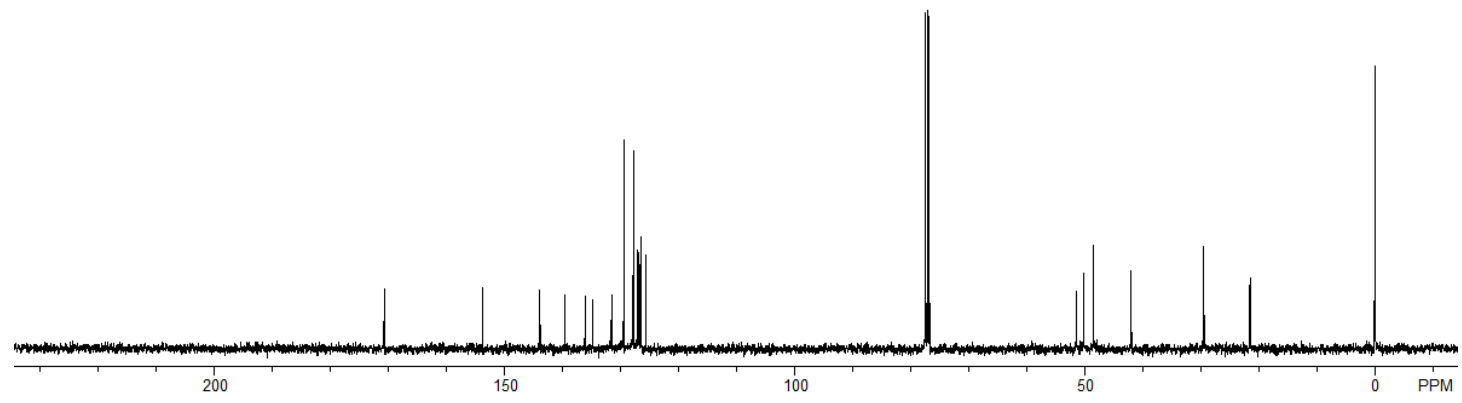




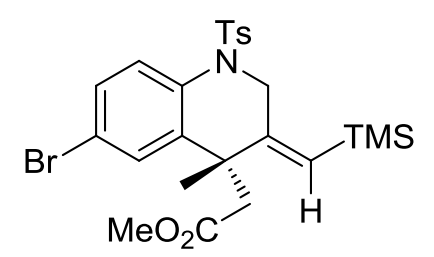

2g
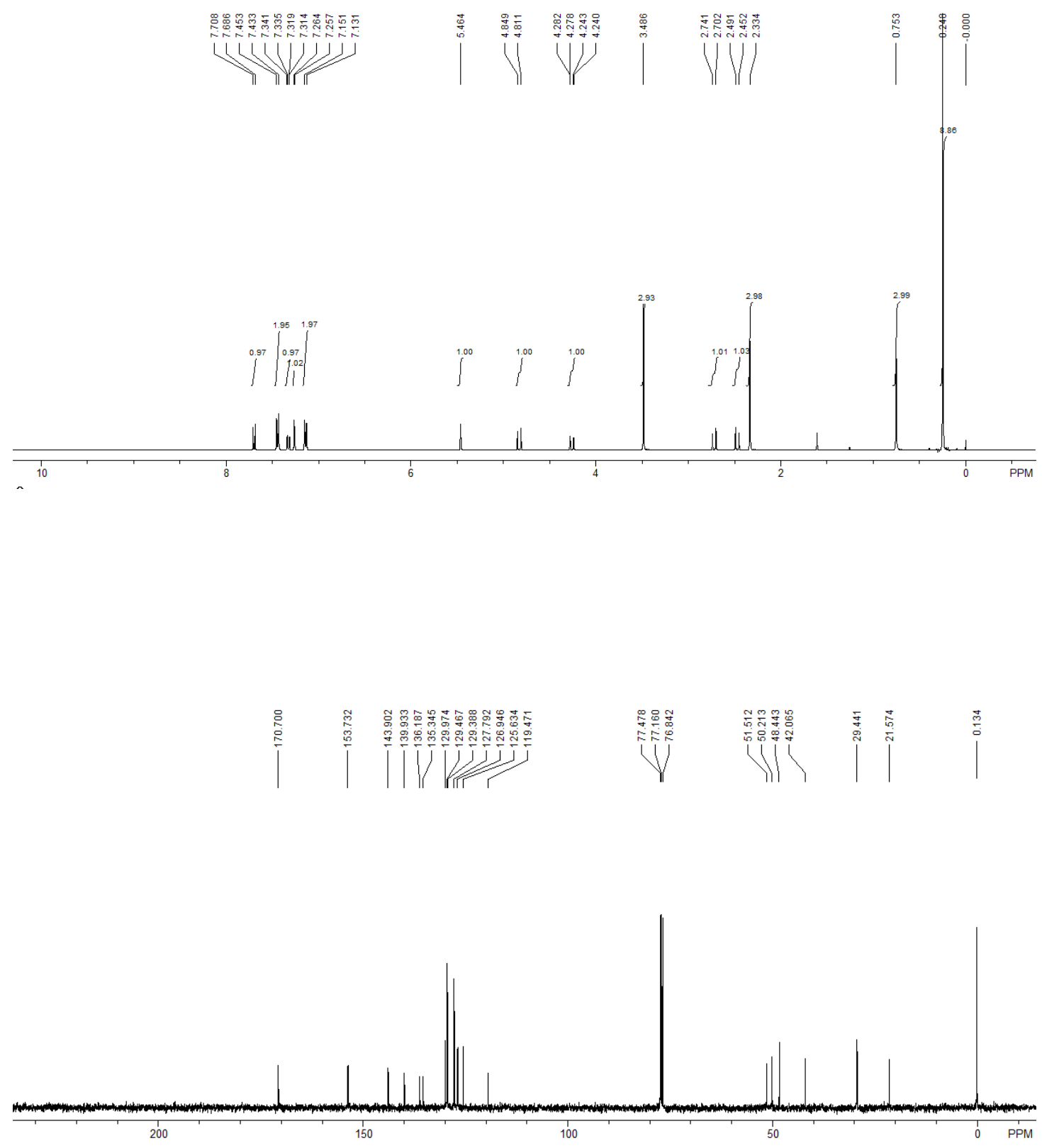
<smiles>CC(=O)C[C@]1(C)C(=C(C)C)CN([As])c2ccc(C(F)(F)F)cc21</smiles>

2h

|l
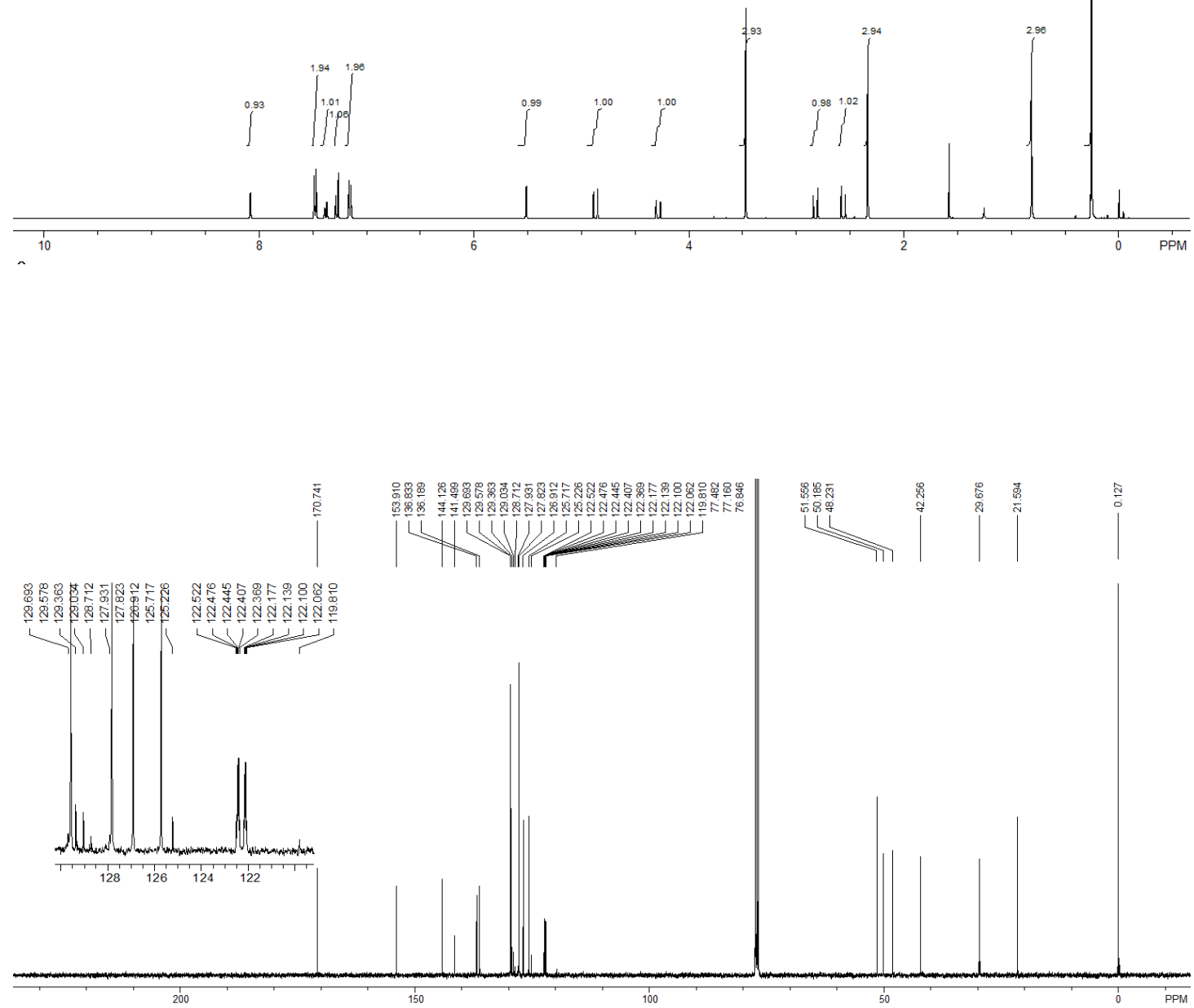


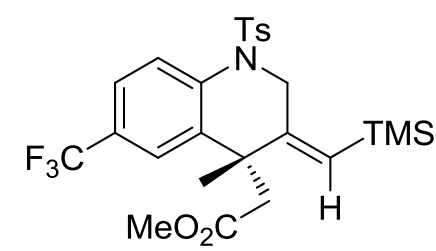

$2 \mathrm{~h}$

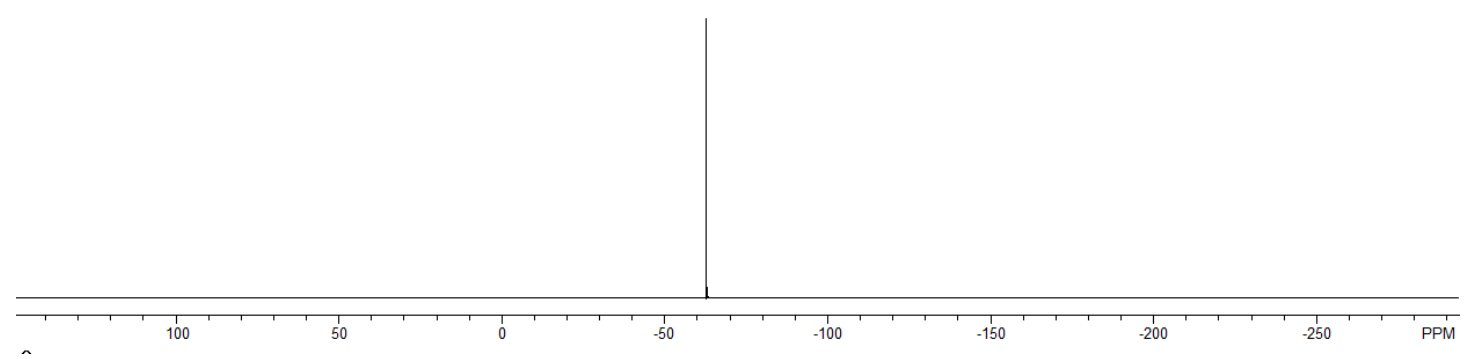




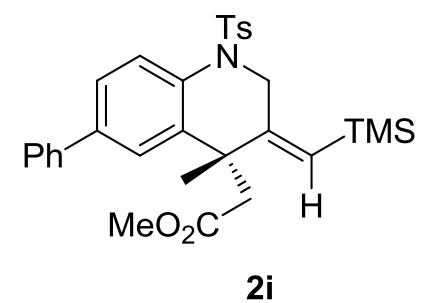

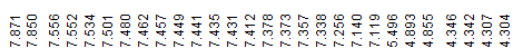

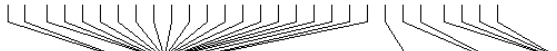
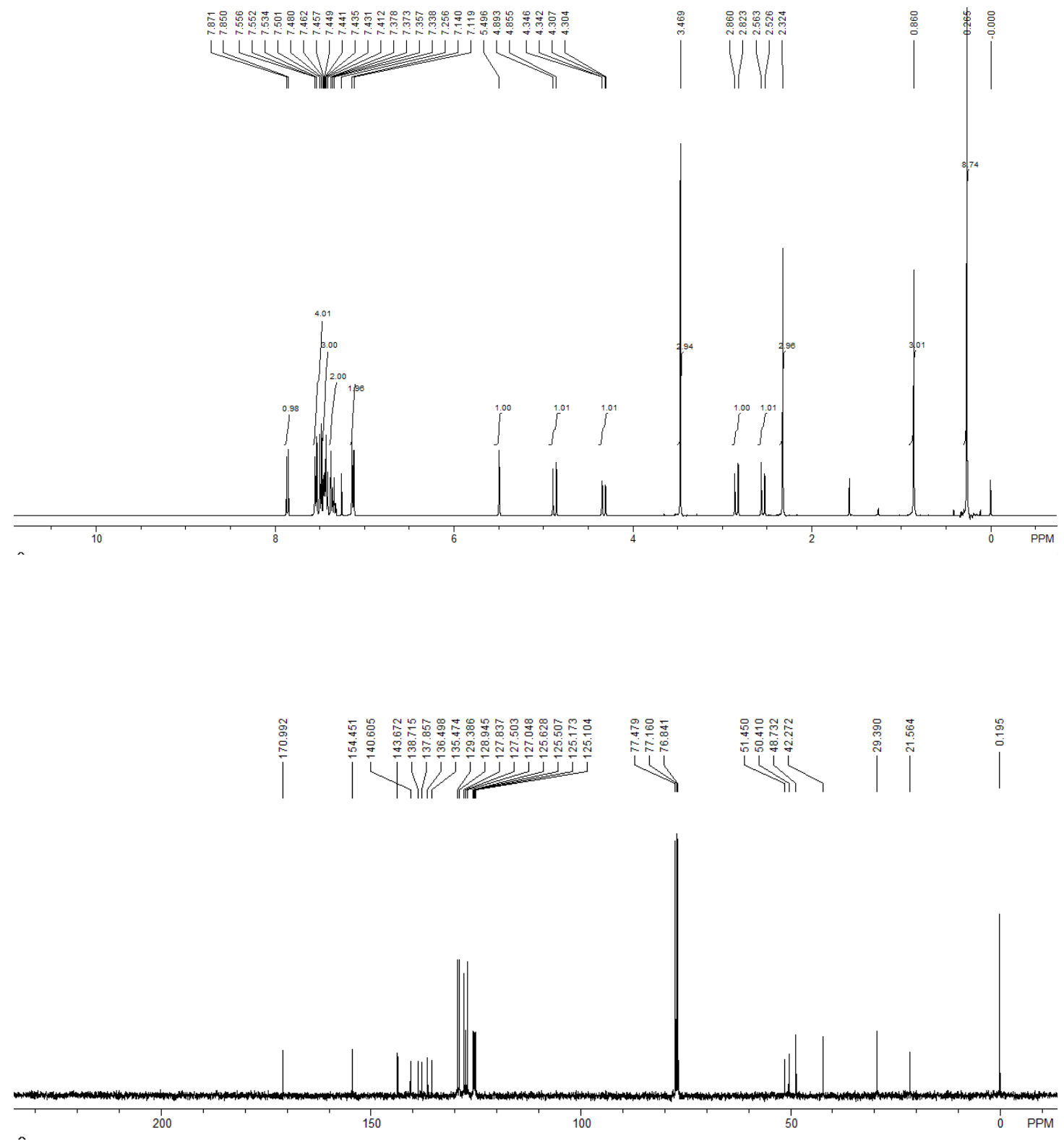
<smiles>CC(=O)C[C@@]1(C)/C(=C/OC(C)=O)CN([AlH2])c2ccccc21</smiles>

2j
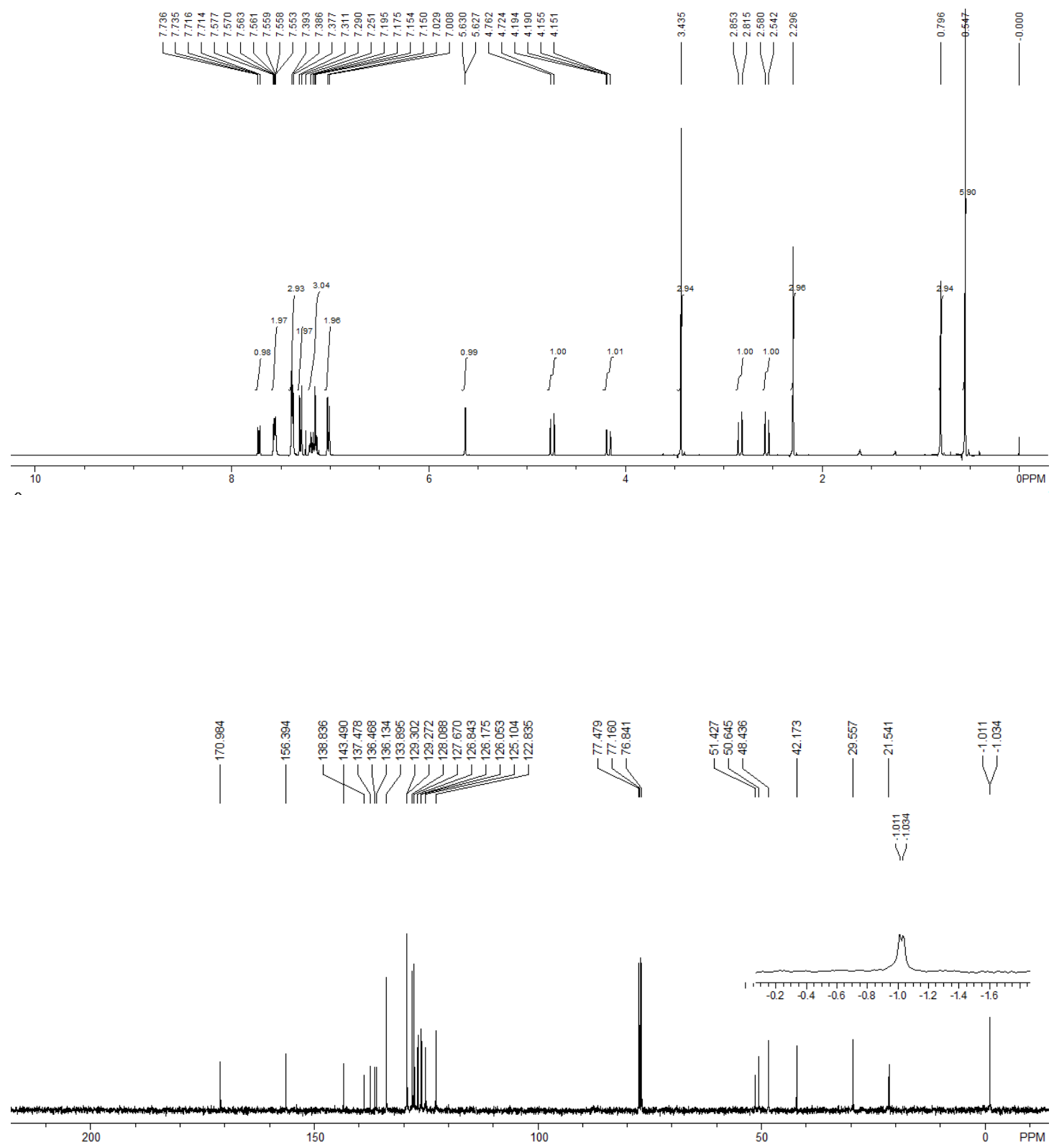

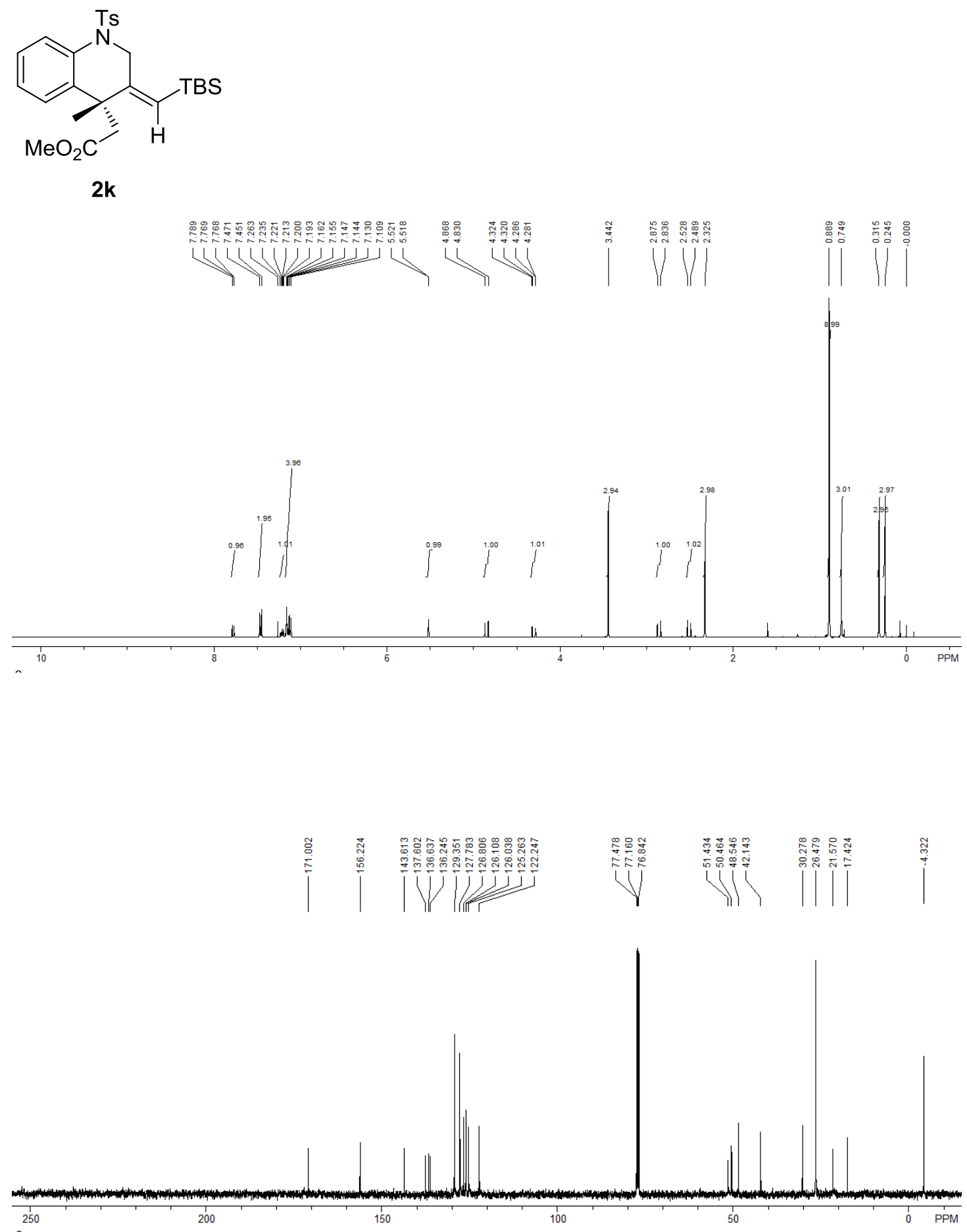


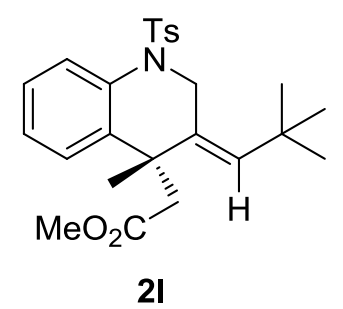

|
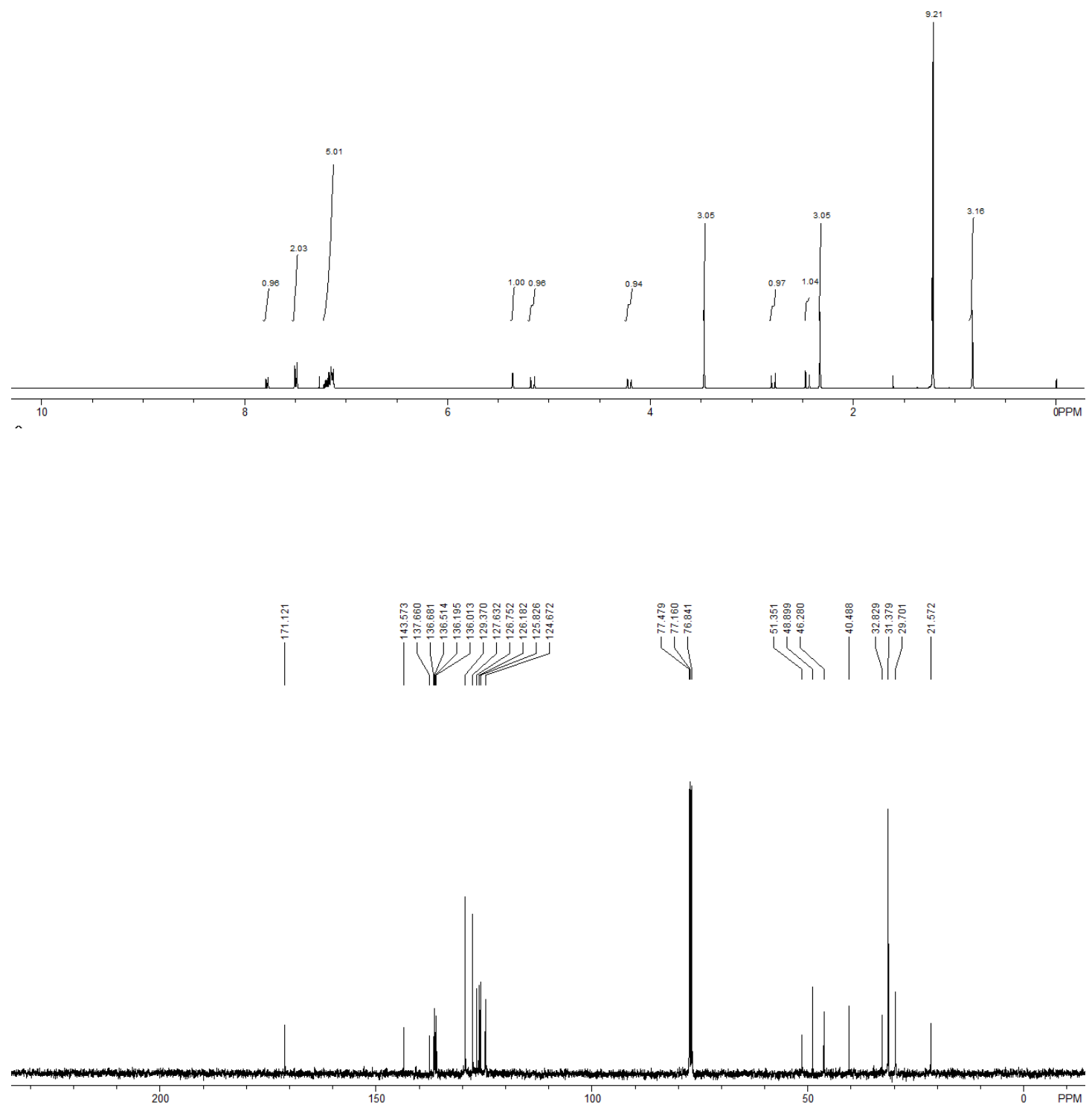

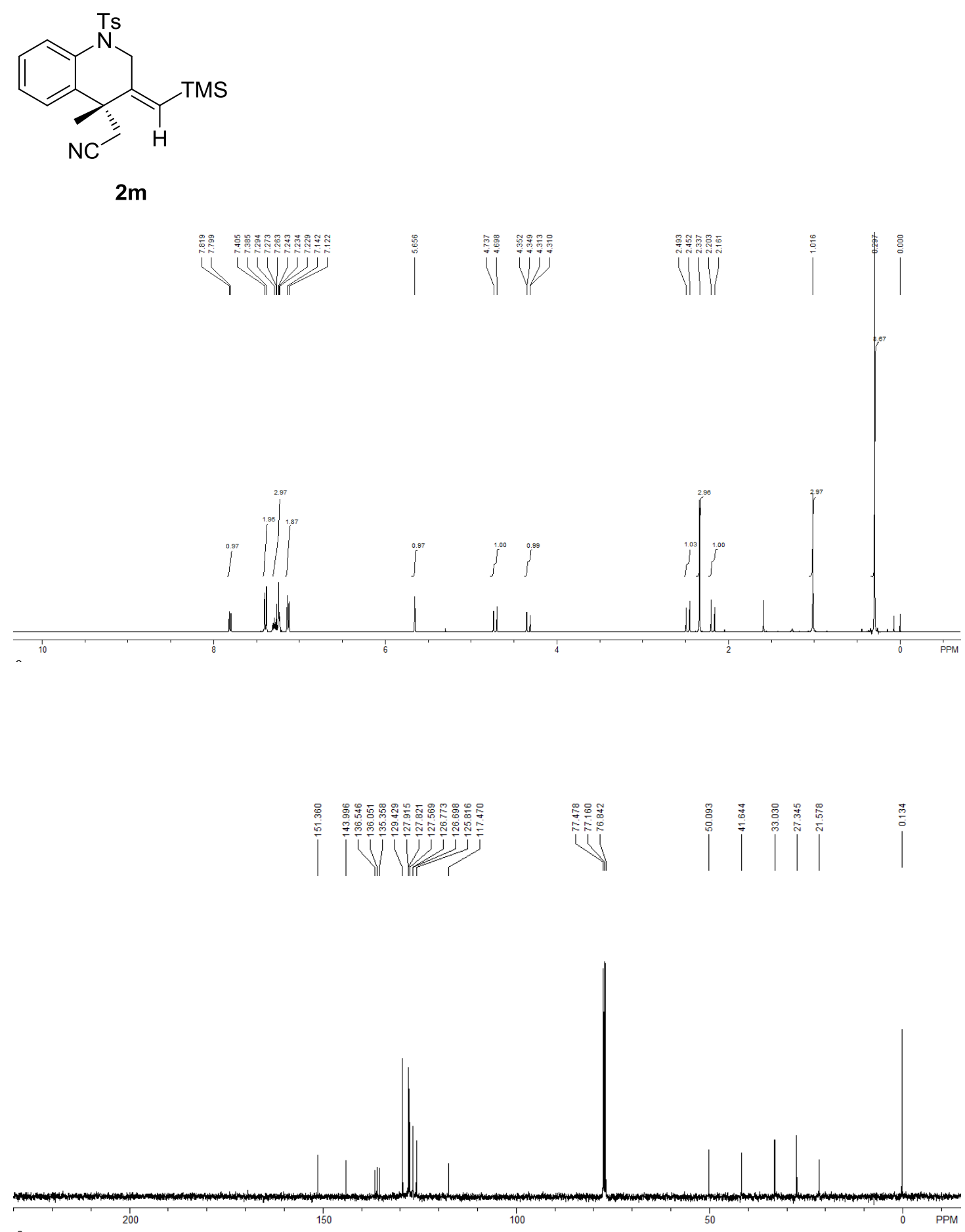
<smiles>CC(=O)C[C@@]1(C)/C(=C/[13CH])CN([As])c2ccccc21</smiles>
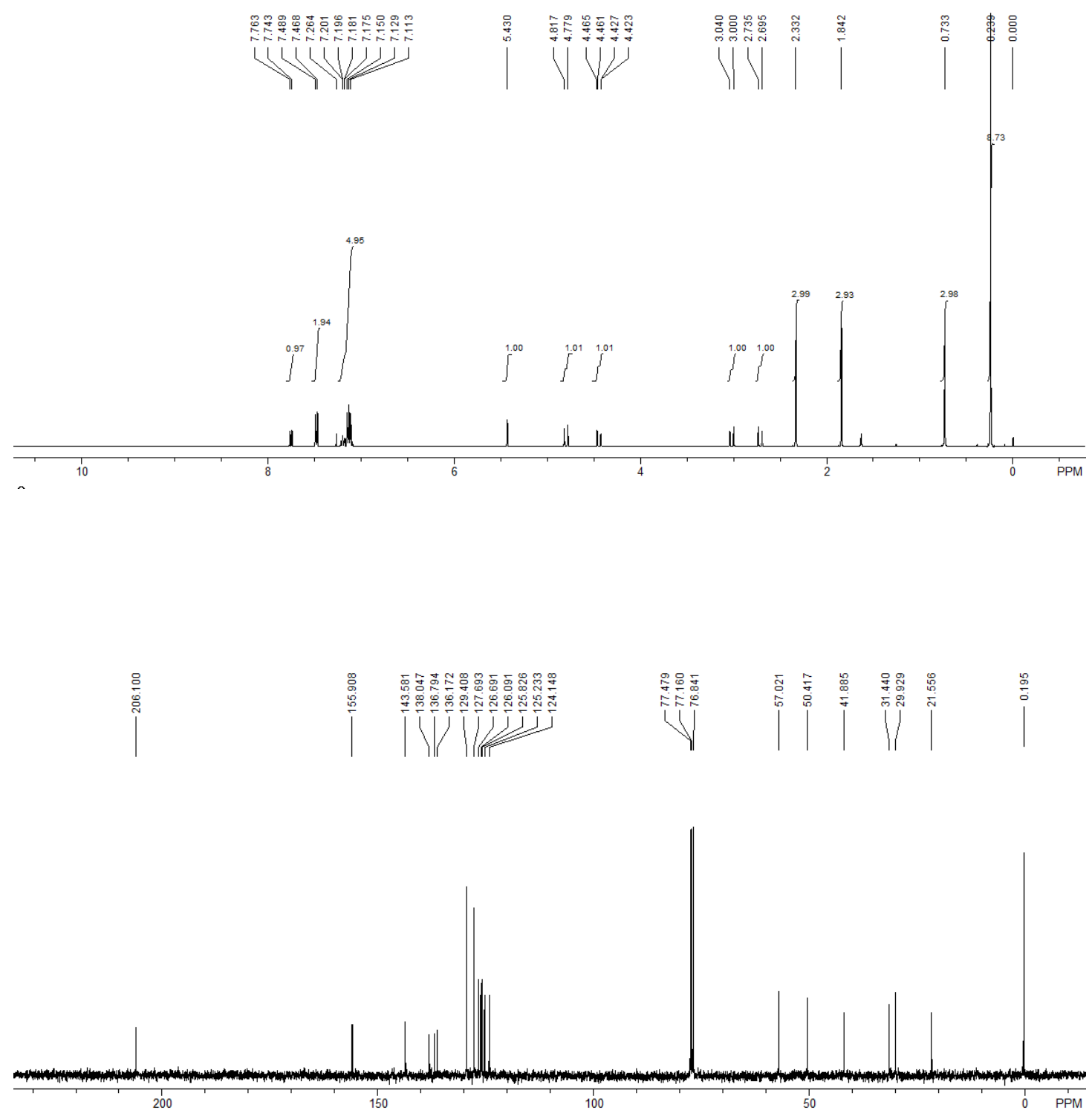
<smiles></smiles>

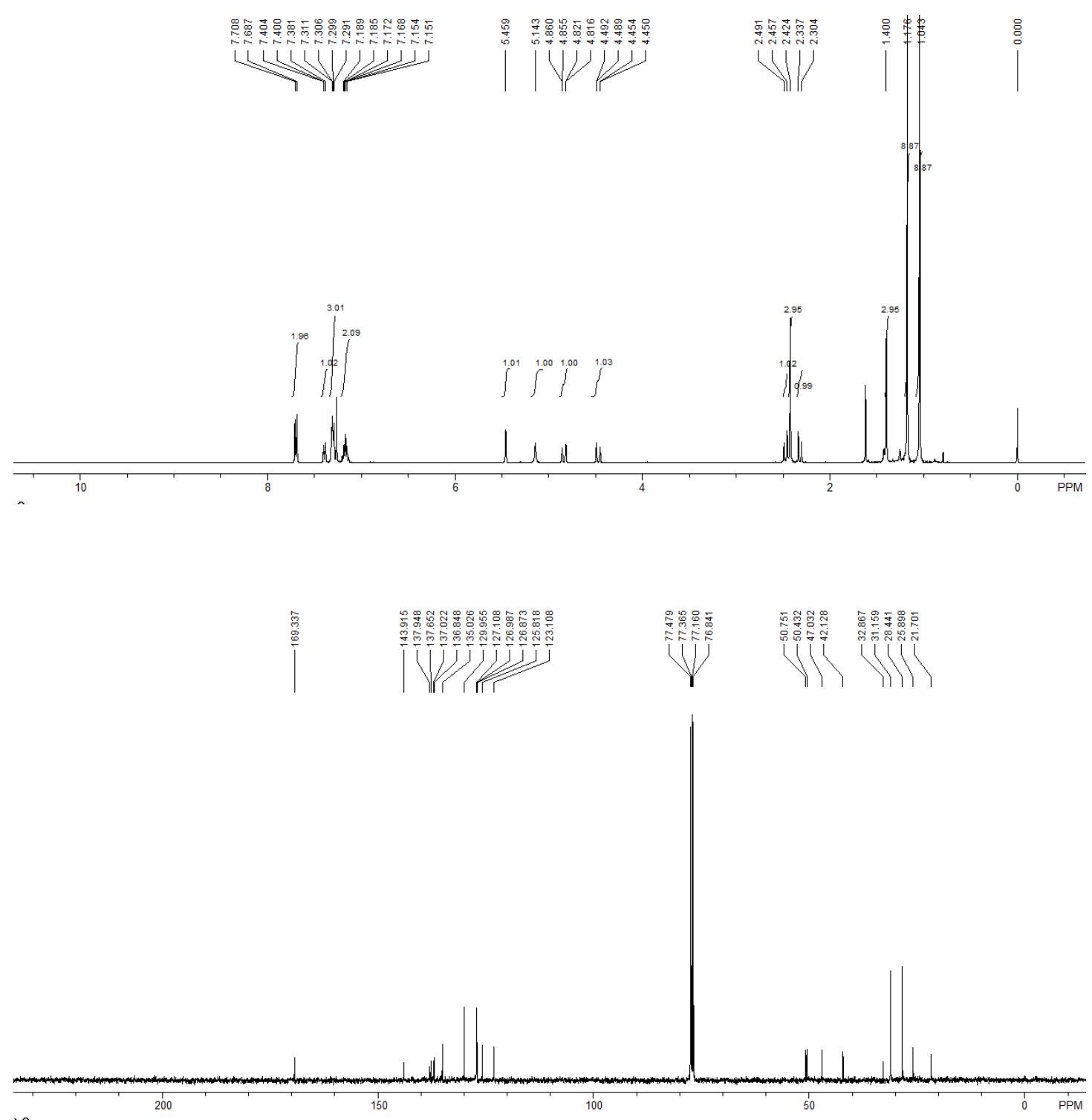




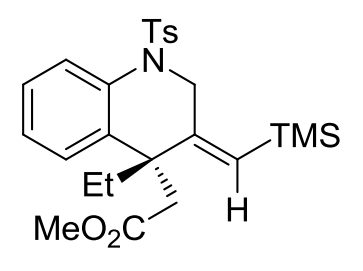

$2 p$
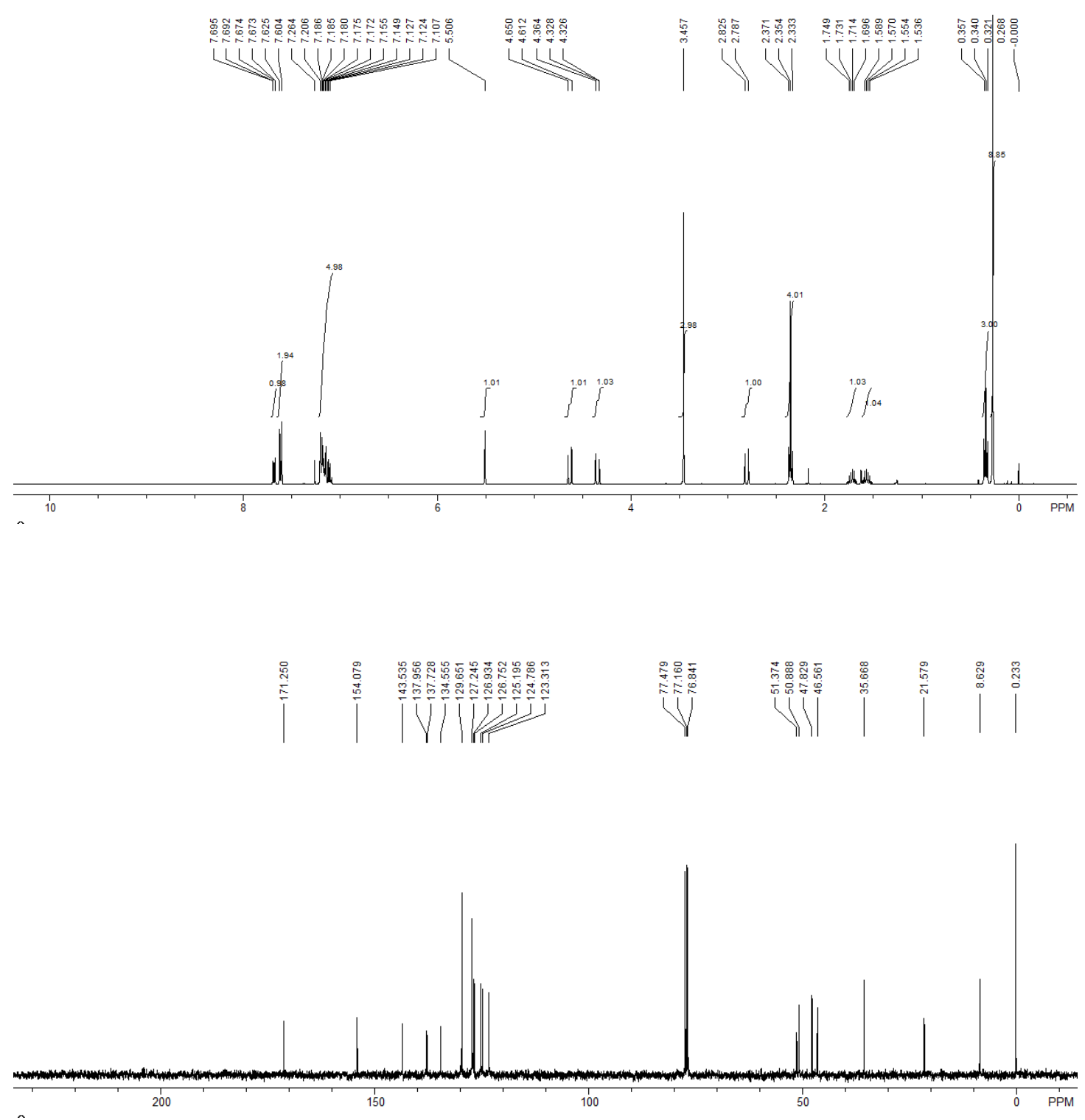


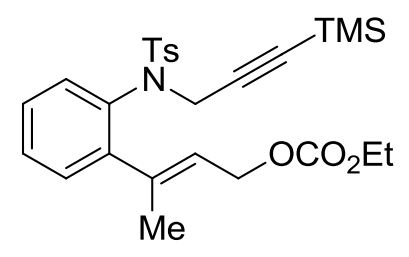

3a

声蛋

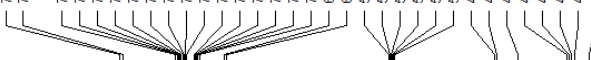

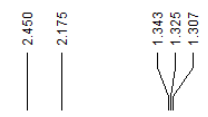

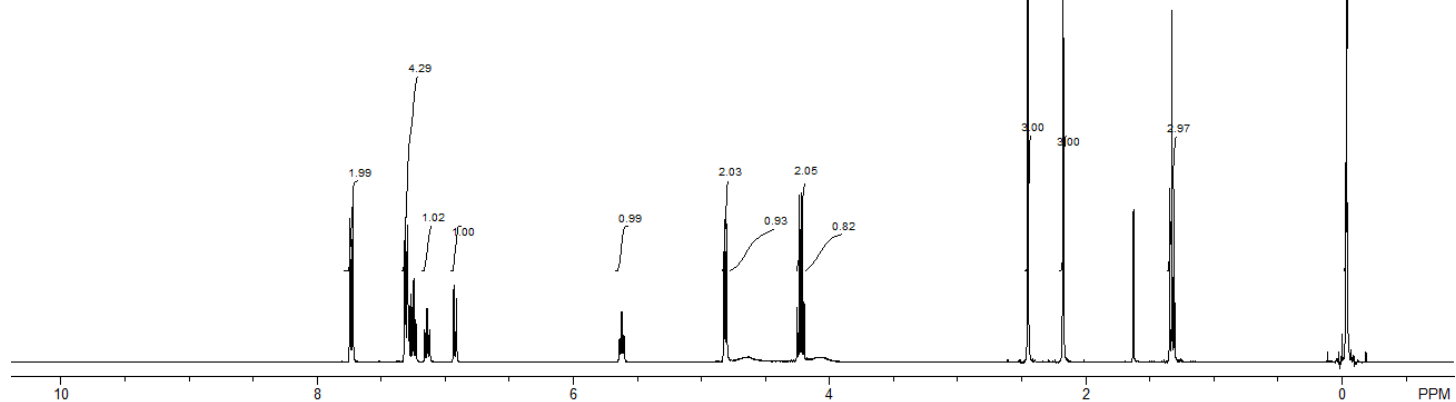

|

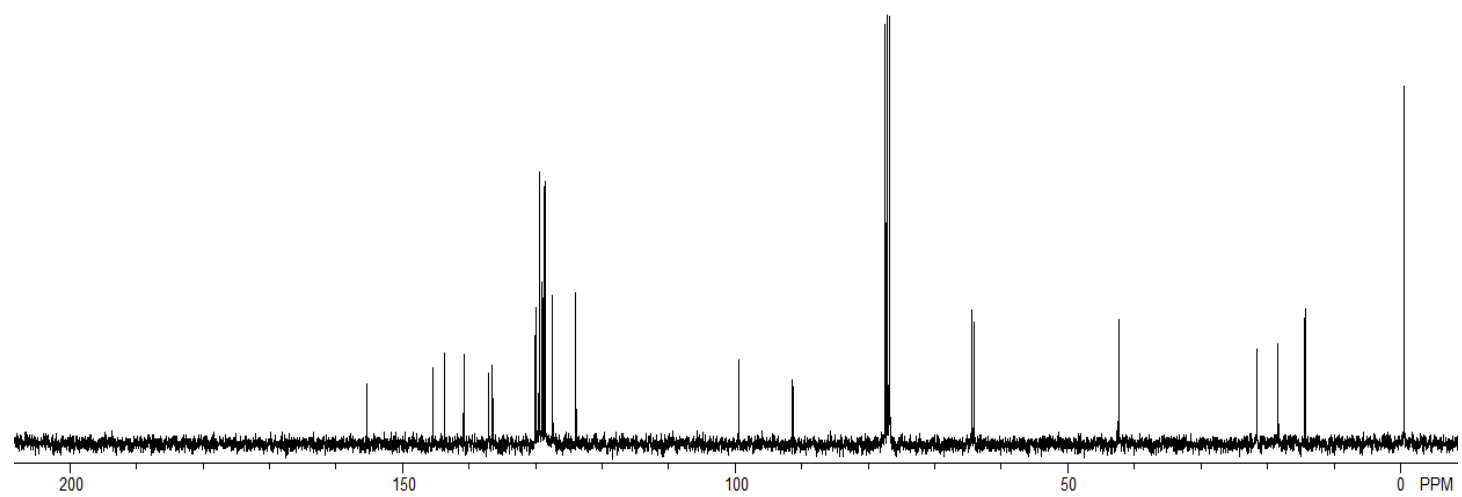


<smiles>[3H]N(CC#CC(C)(C)C)c1ccc(C)cc1/C(C)=C/CCOCC</smiles>
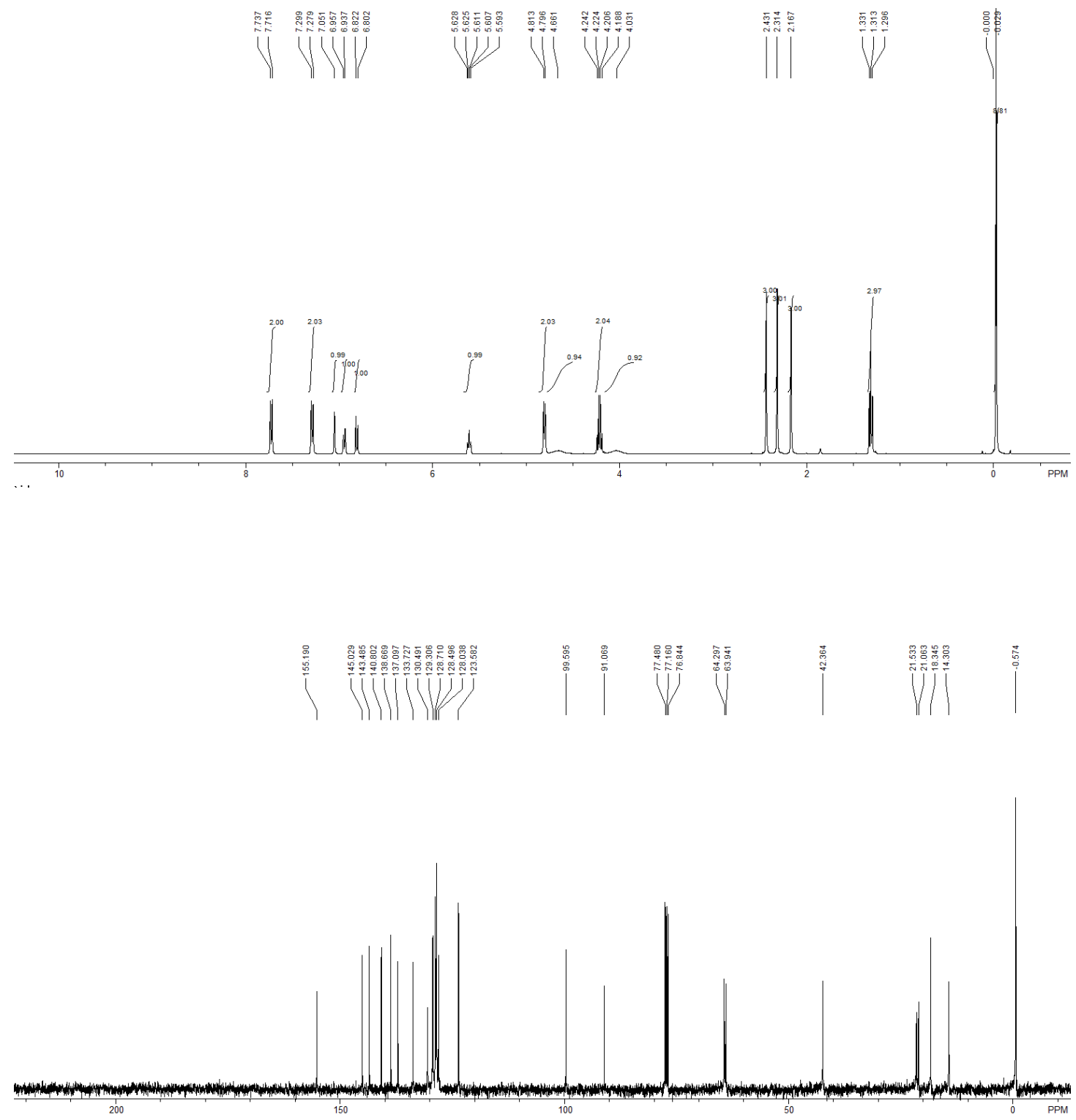

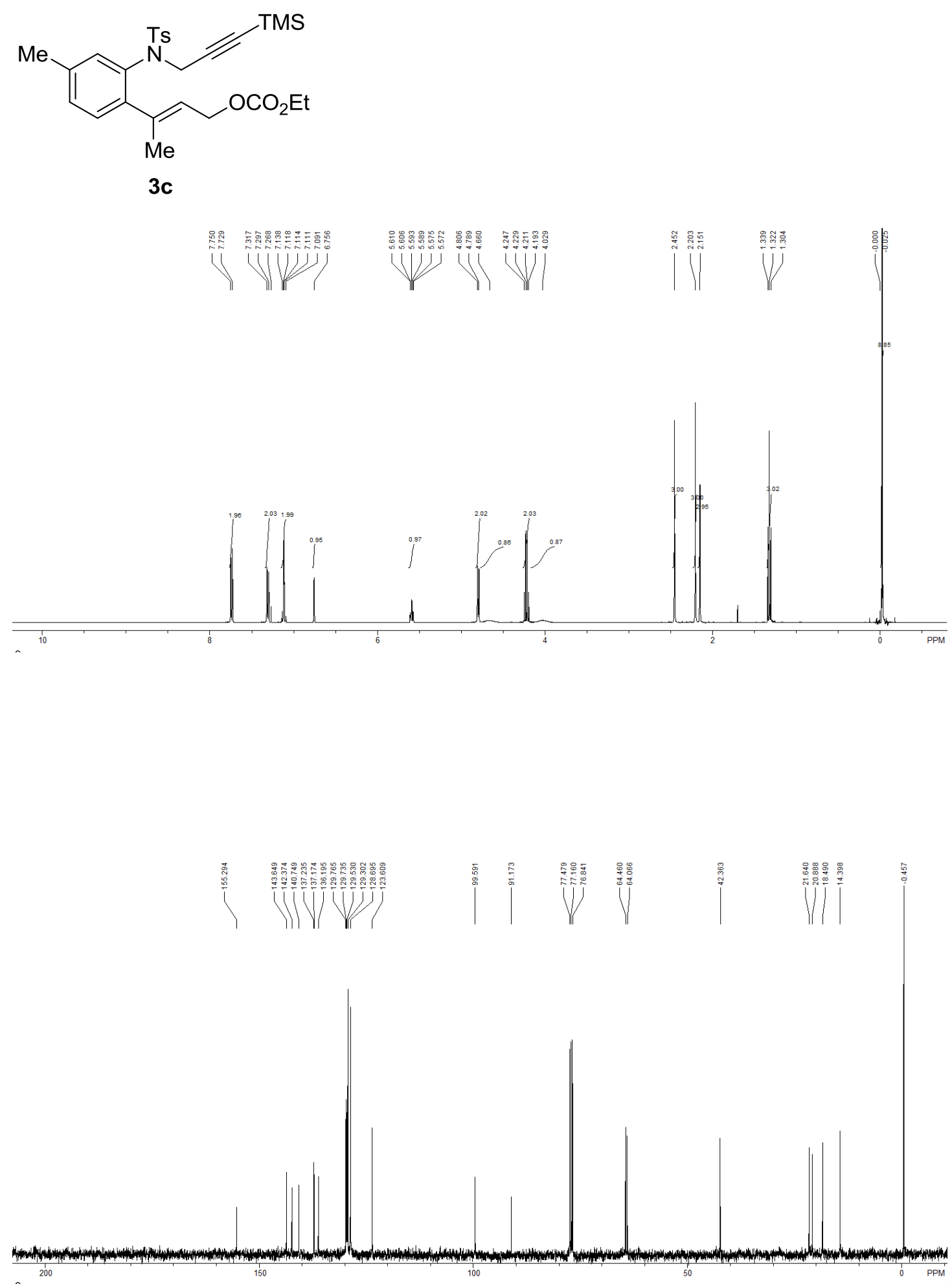

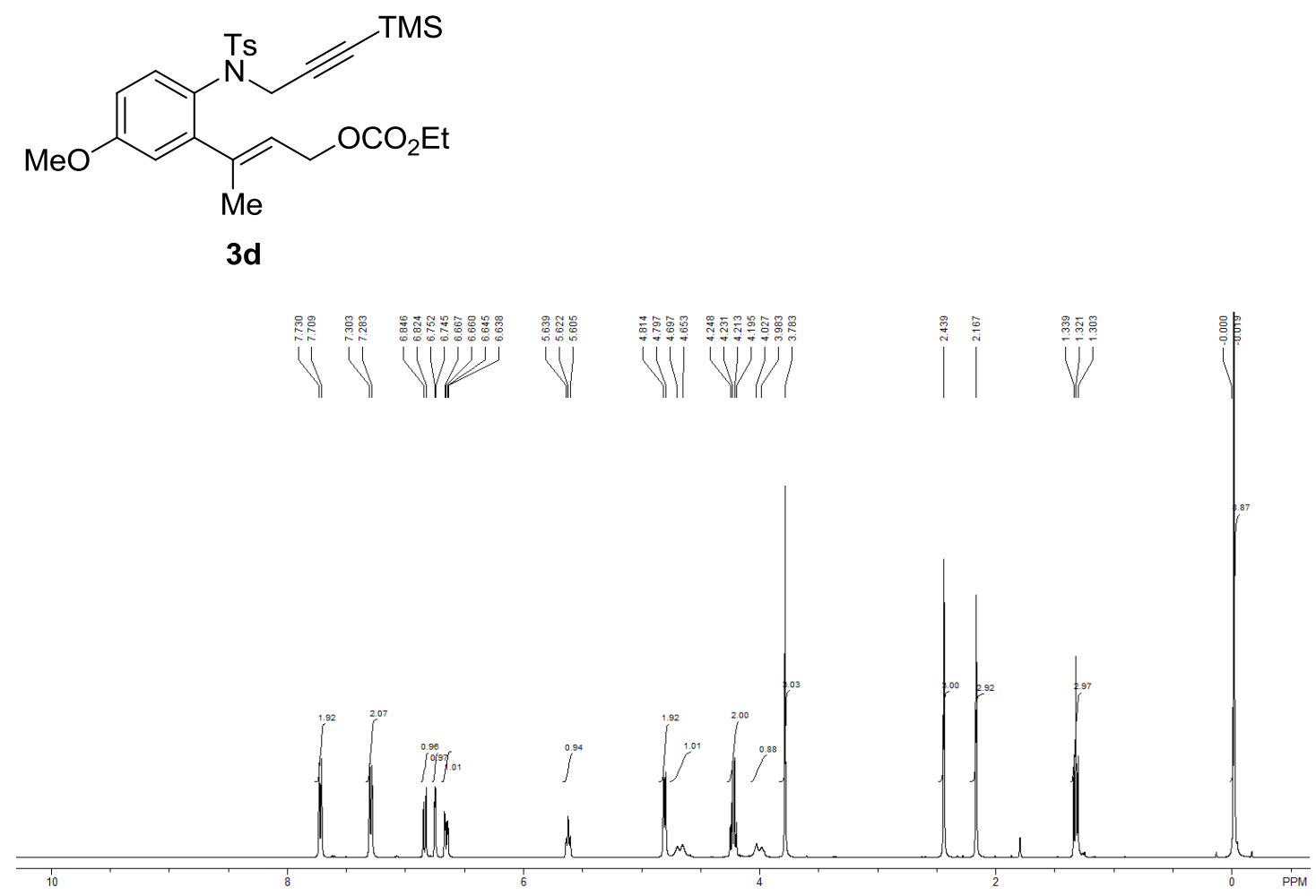

$$
\text { HIIII }
$$

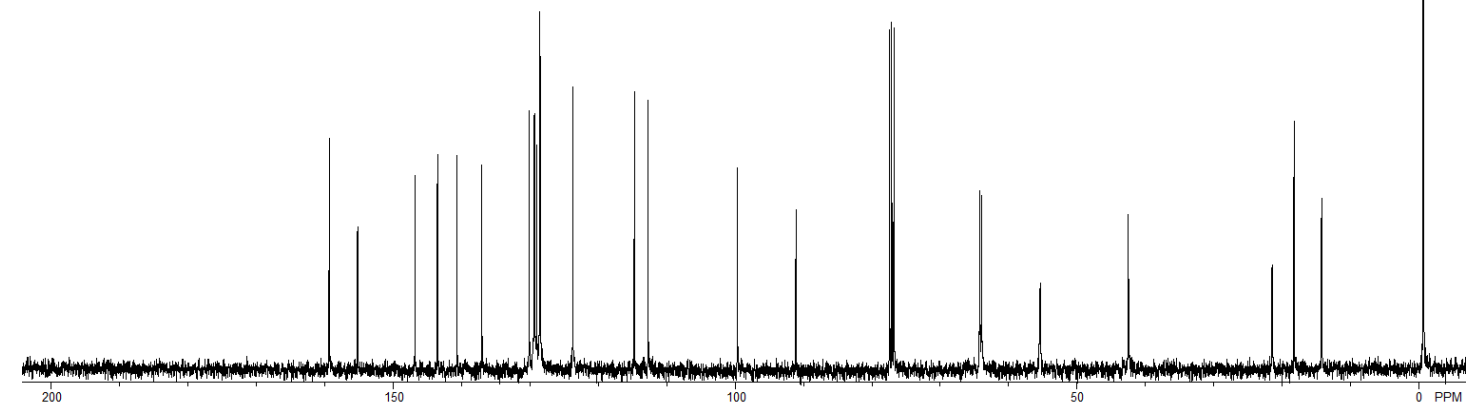


$\overbrace{\mathrm{Me}}^{\mathrm{TMS}}$

$3 e$

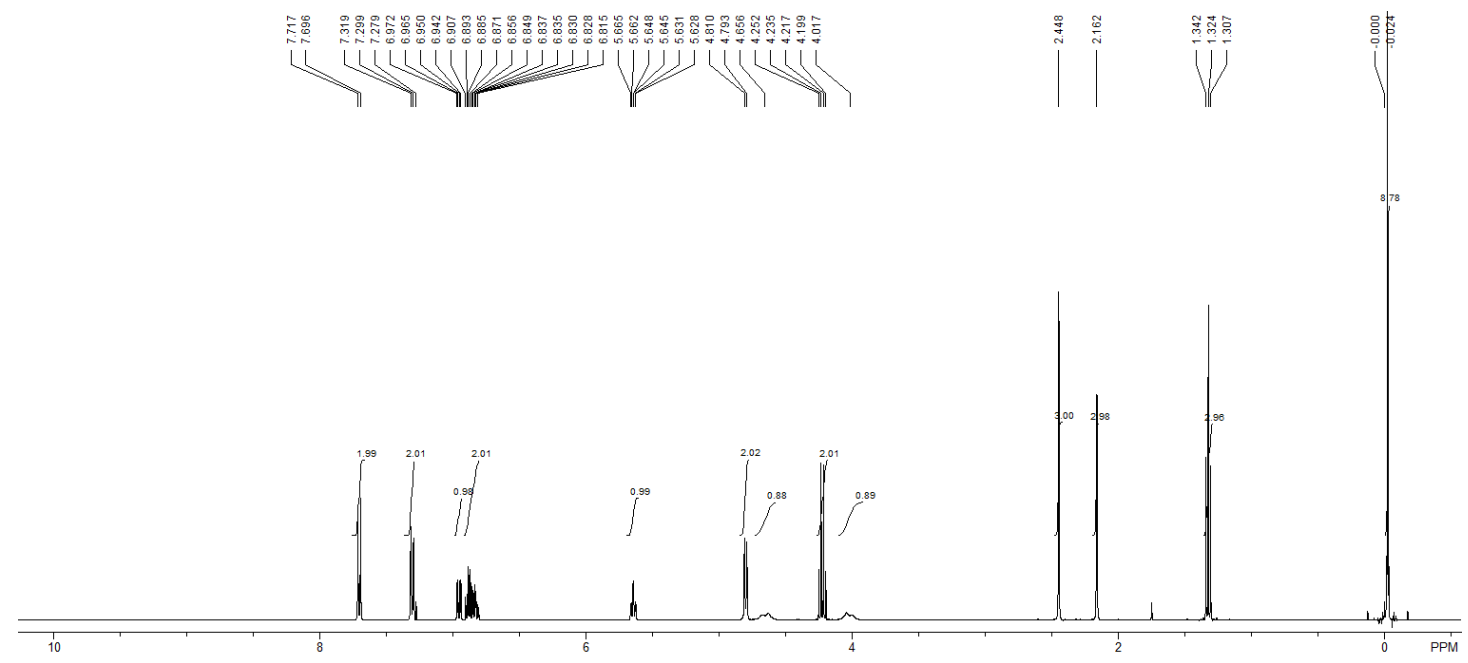

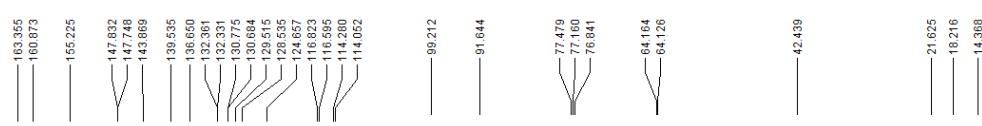

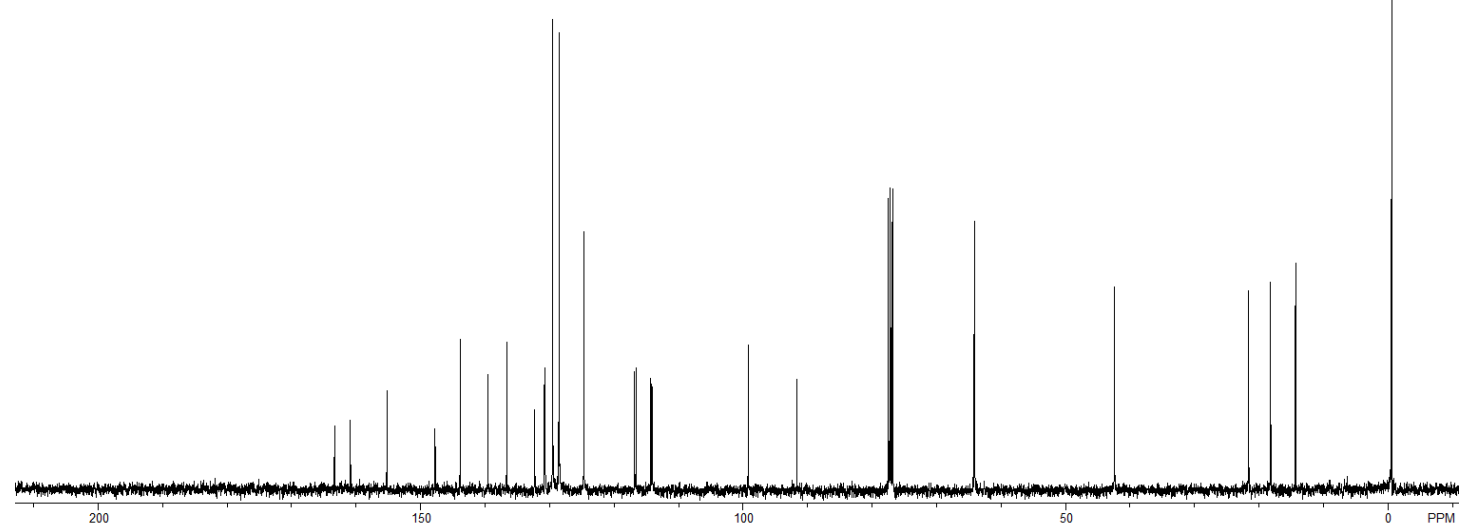




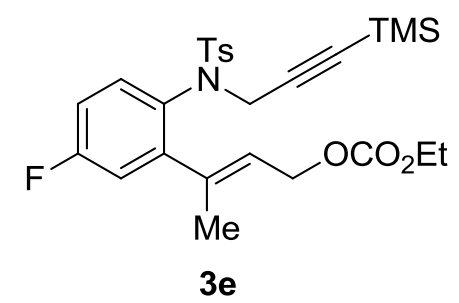

諅

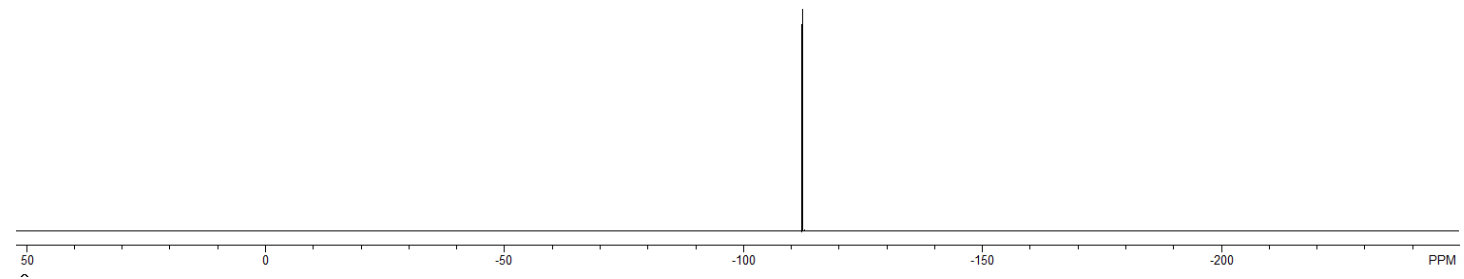


<smiles>[3H]N(CC#CC(C)(C)C)c1ccc(Cl)cc1/C(C)=C/COC(=O)OCC</smiles>

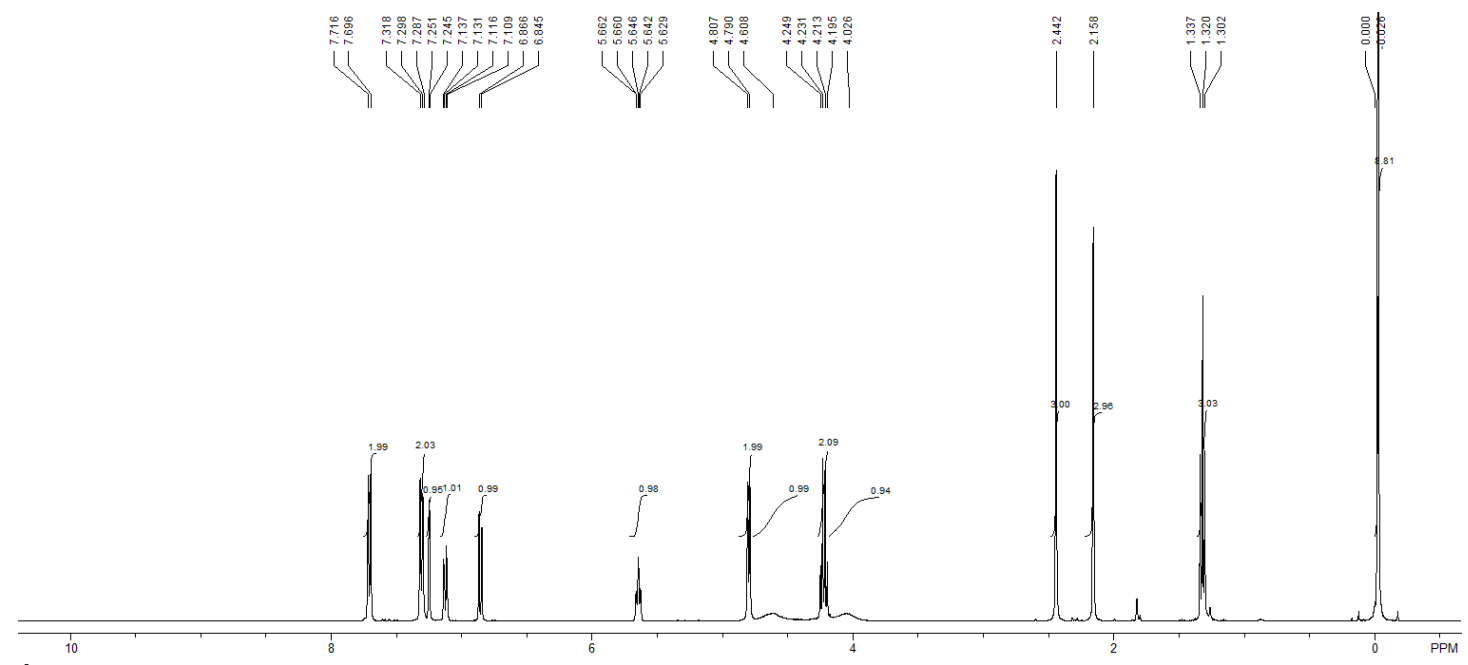

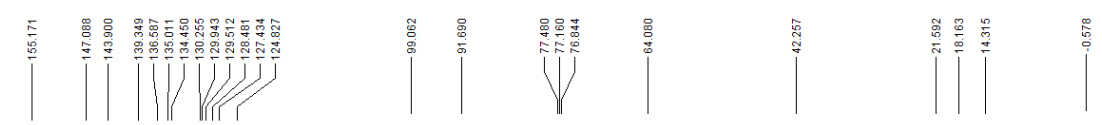

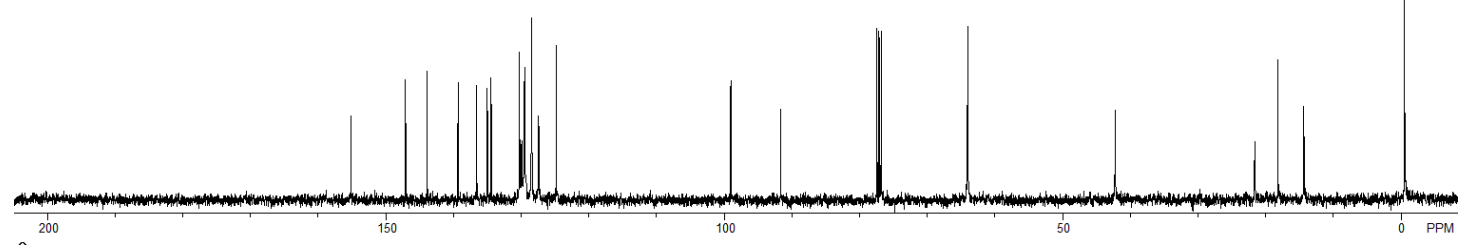



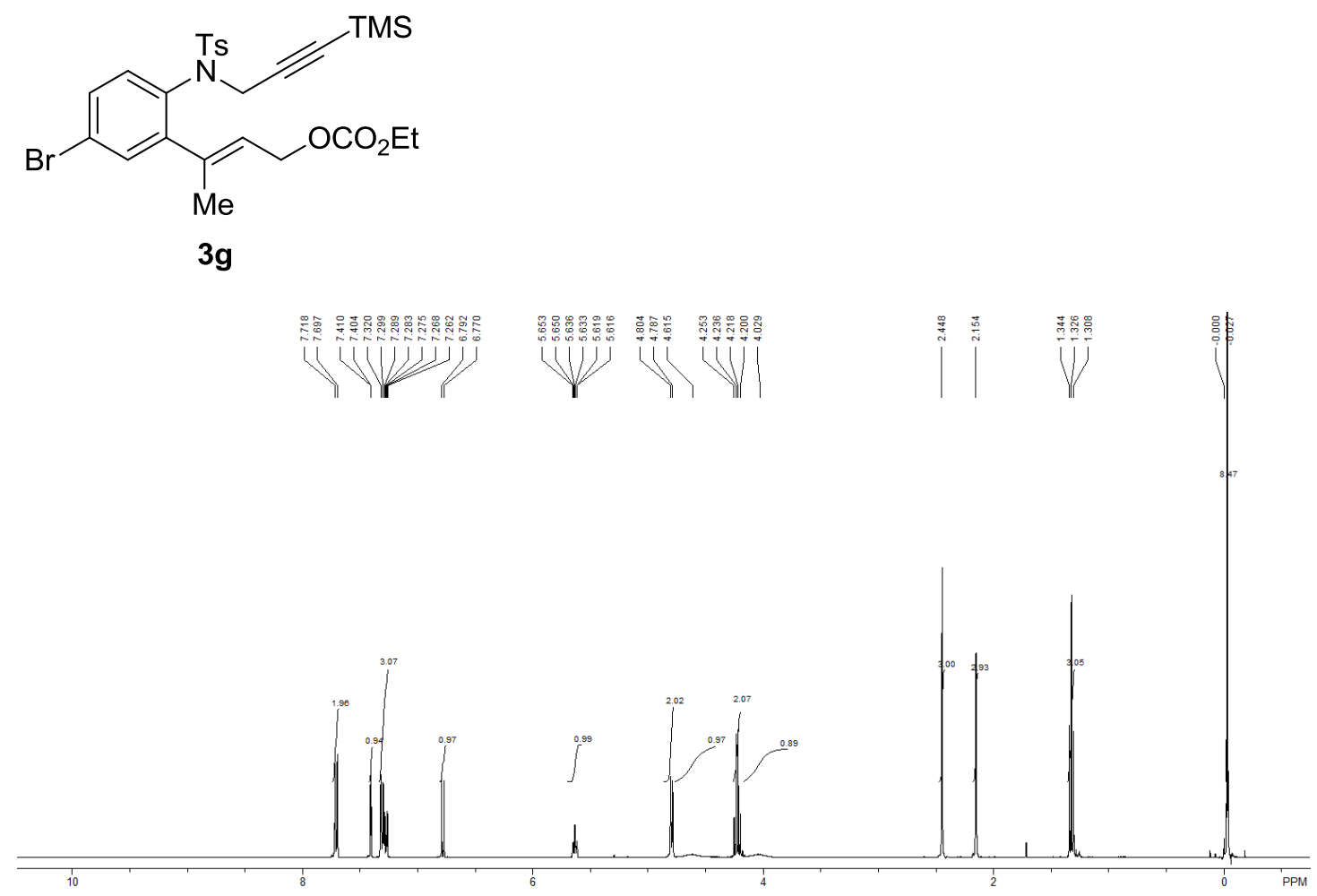

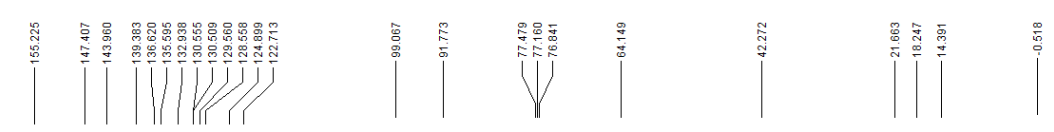

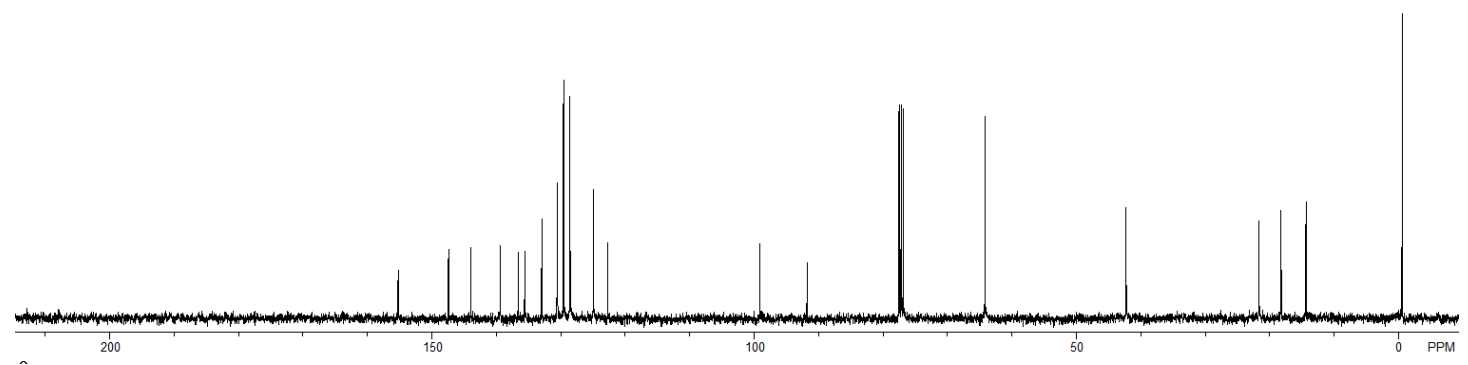


<smiles>CCOCOCC=C(C)c1cc(C(F)(F)F)ccc1N([37F])CC#CC(C)(C)C</smiles>

3h
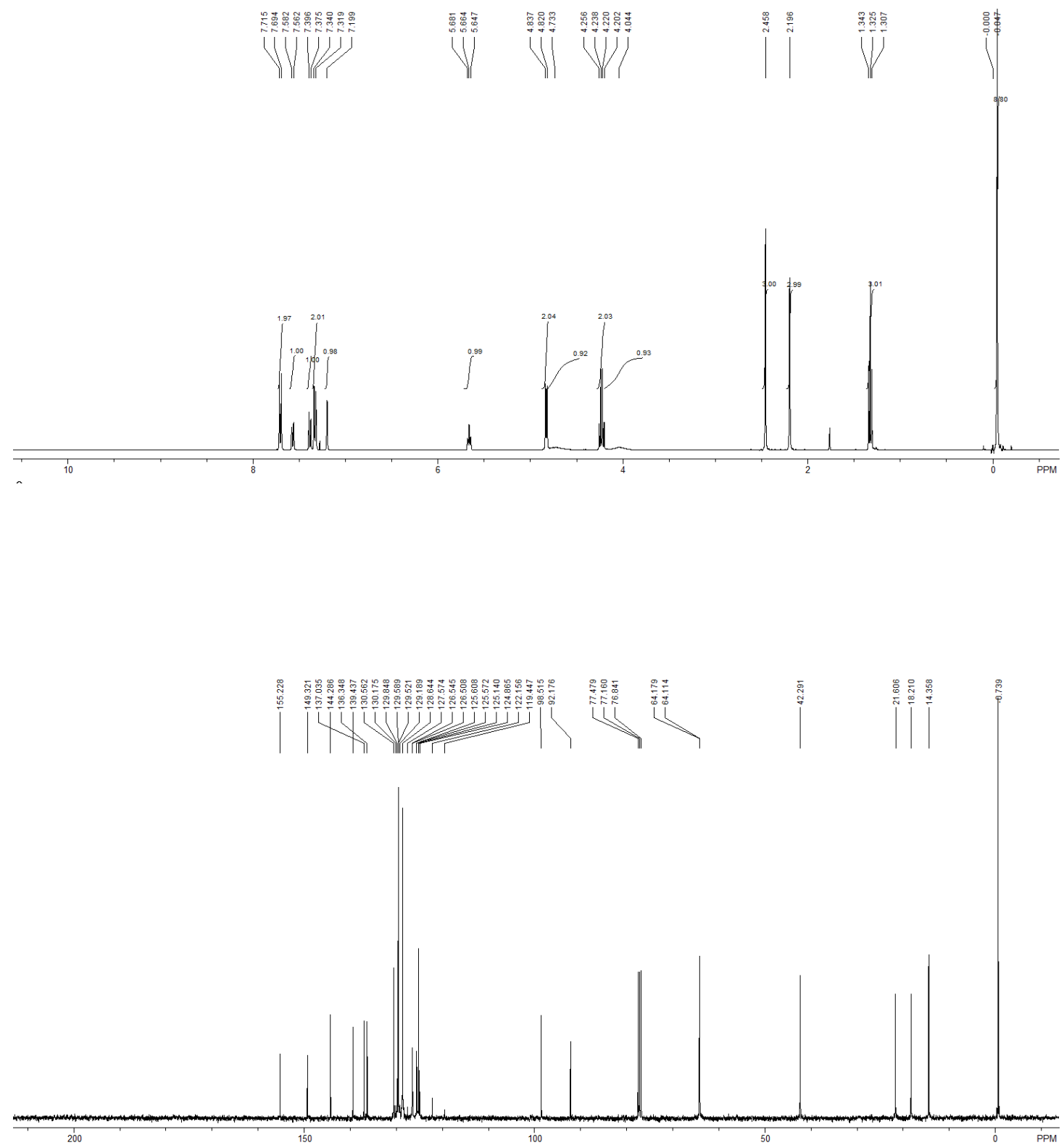
<smiles>[Y5]N(CC#CC(C)(C)C)c1ccc(C(F)(F)F)cc1/C(C)=C/COC(C)C</smiles>

3h

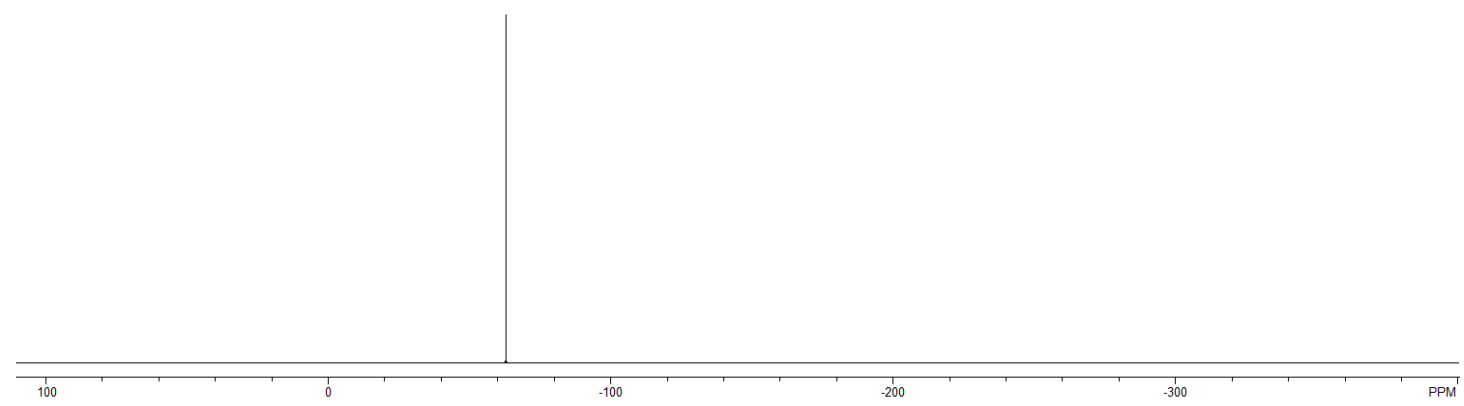




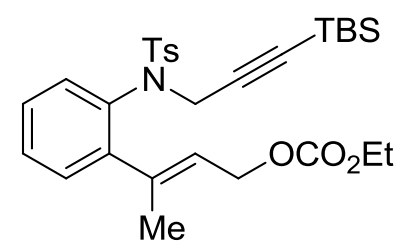

$3 \mathbf{i}$
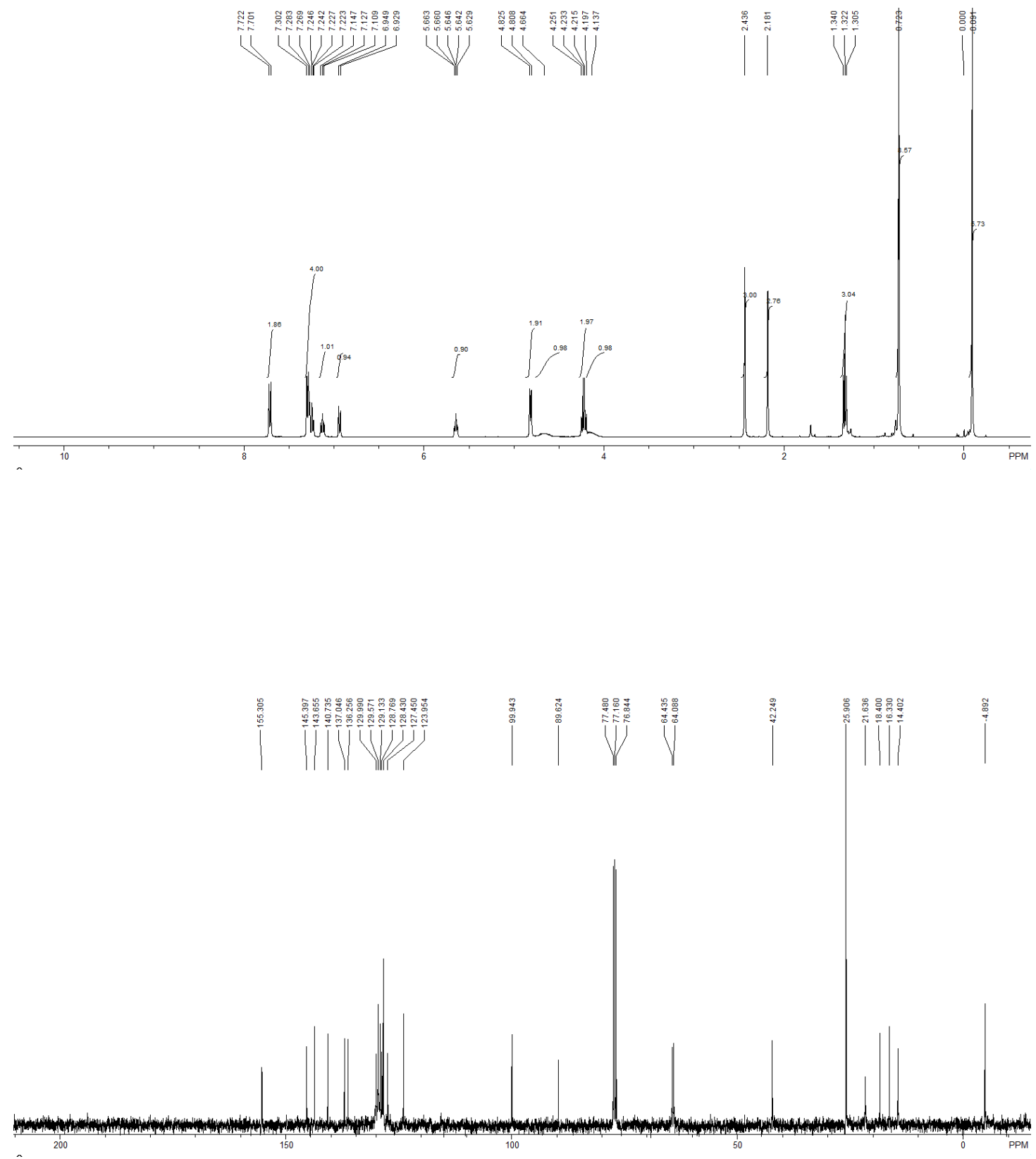


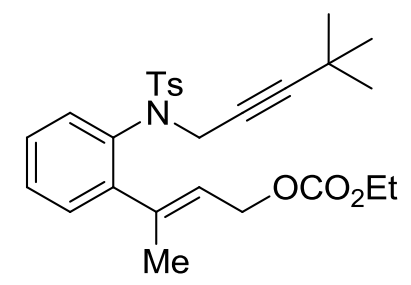

3j

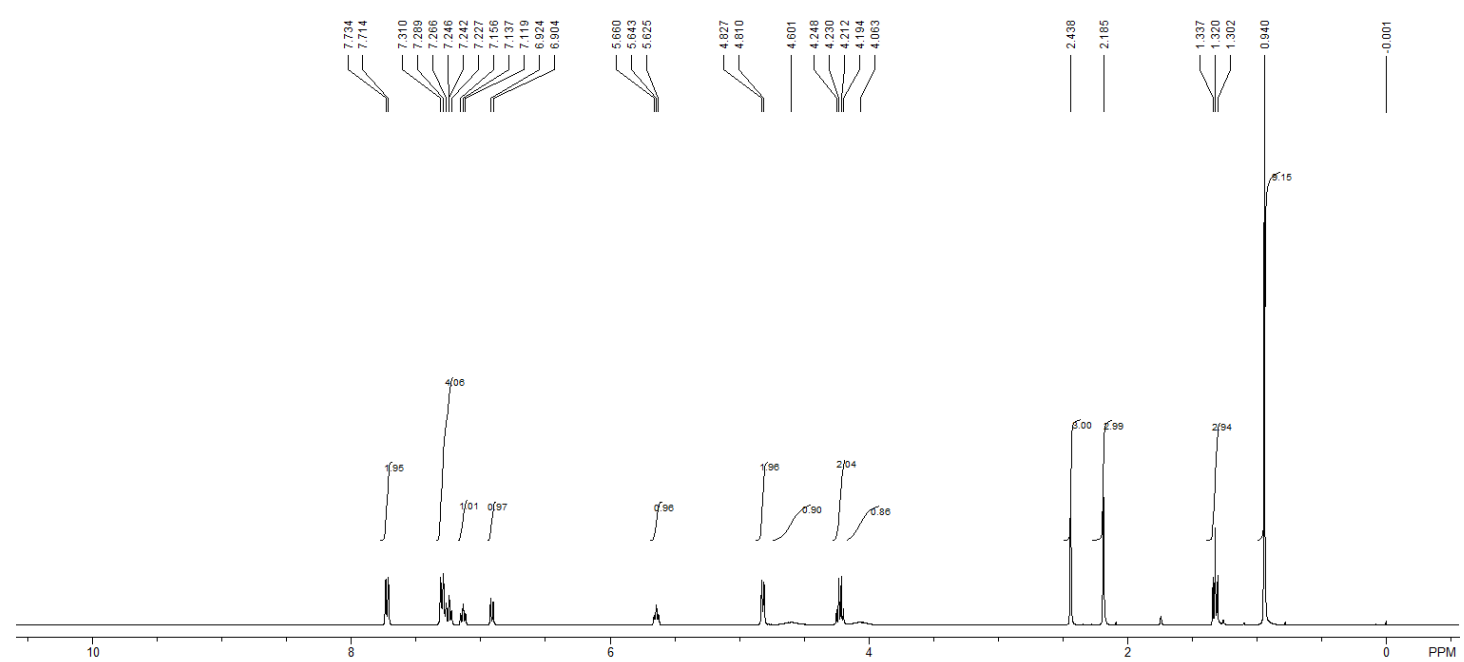


<smiles>C=C[C@]1(C)/C(=C/[13C](C)(C)C)CN([As])c2ccccc21</smiles>

$4 a$
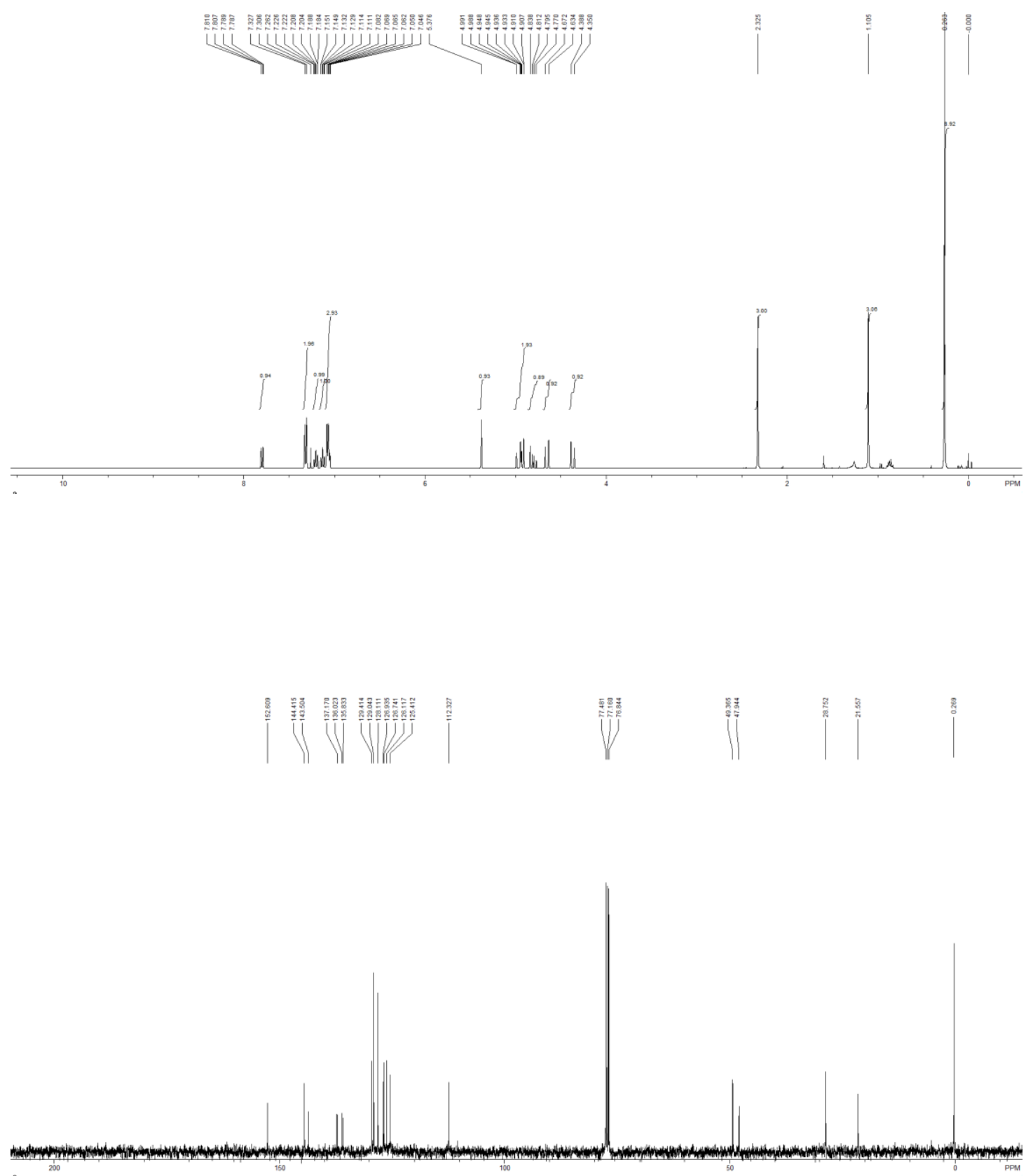
<smiles>C=C[C@]1(C)/C(=C/[Y15](C)(C)C)CN([As])c2ccc(C)cc21</smiles>

4b
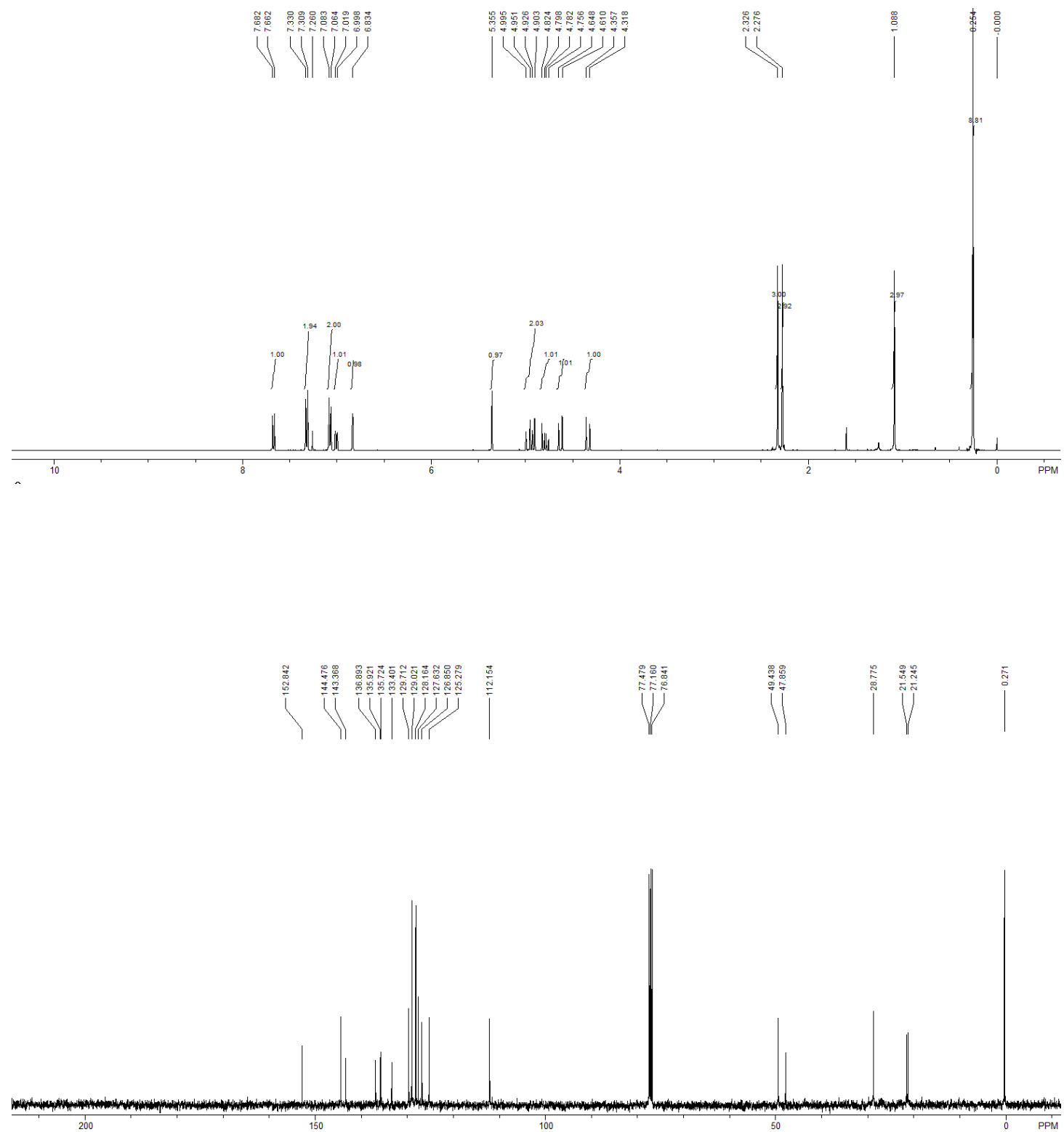


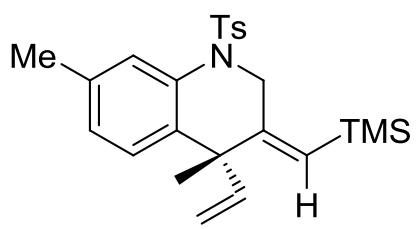

$4 c$

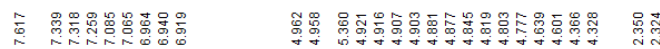

Vivi
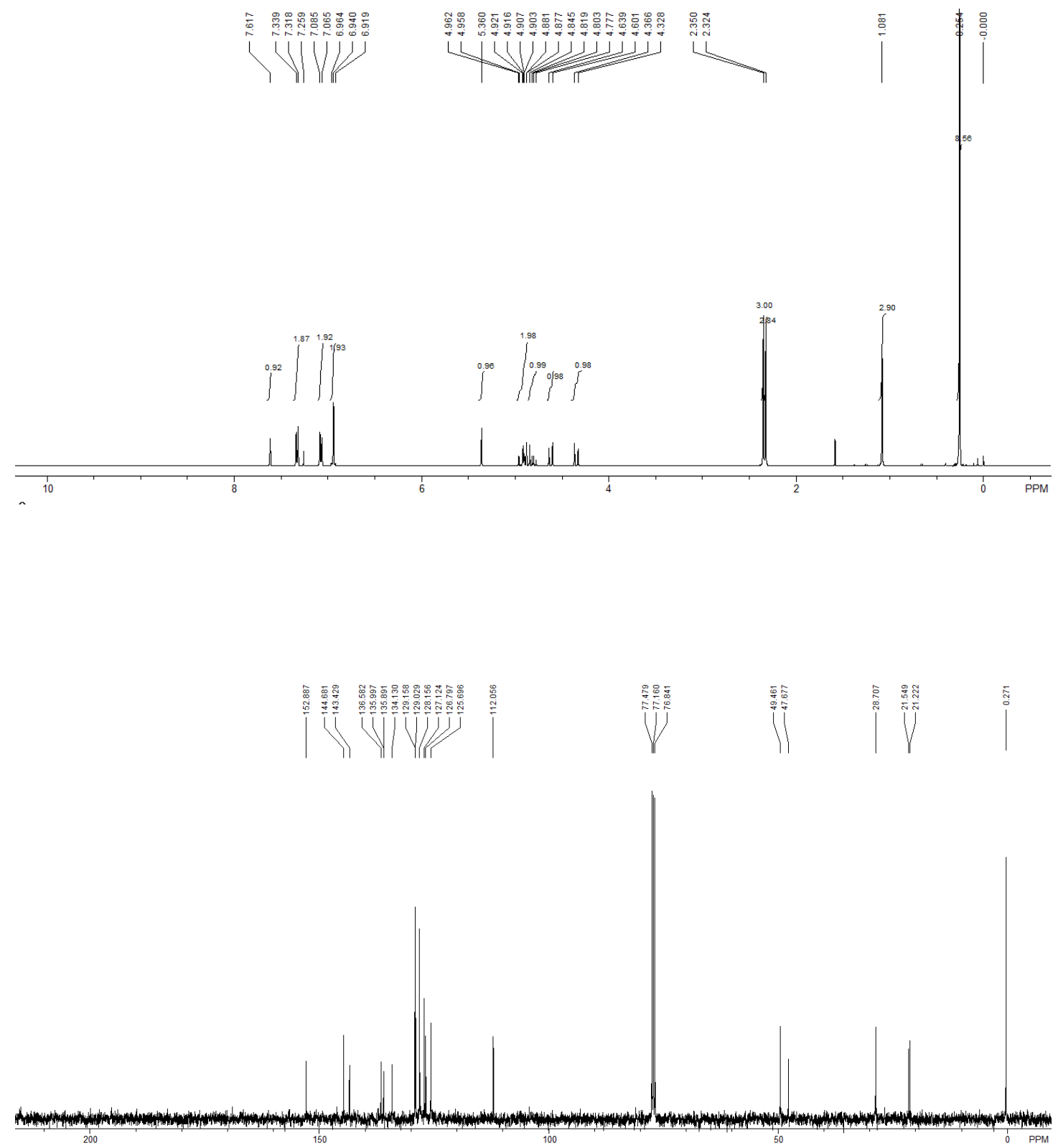
TMS

4d

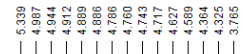

은

WUWH

W Wiflut
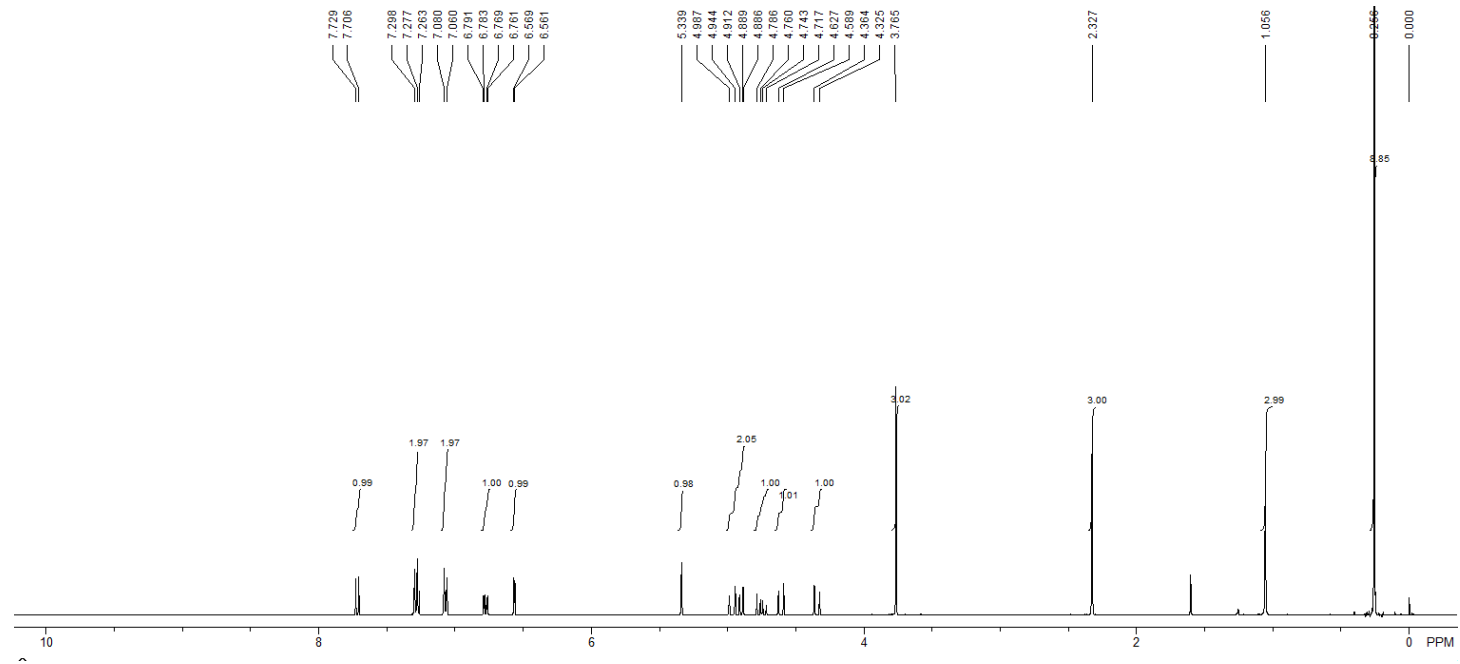

||
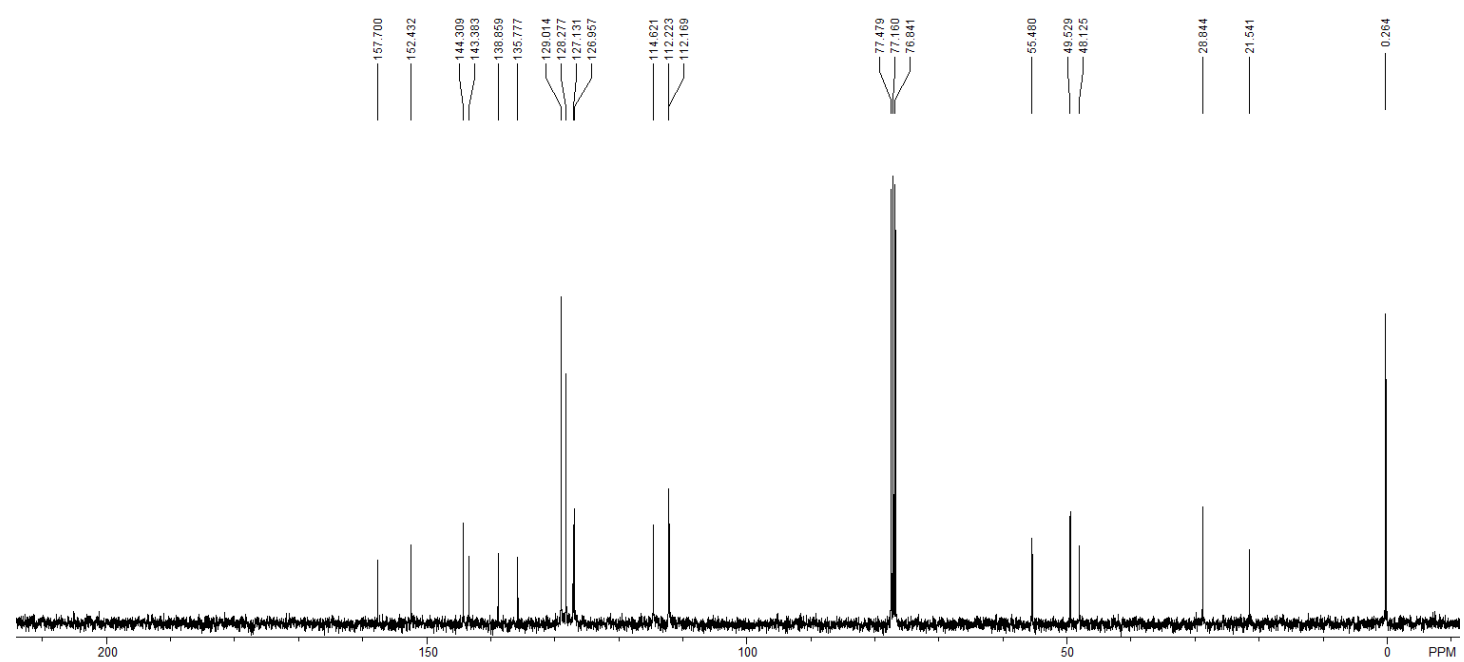
<smiles>C=C[C@]1(C)/C(=C/[Y15](C)(C)C)CN([As])c2ccc(F)cc21</smiles>

$4 e$
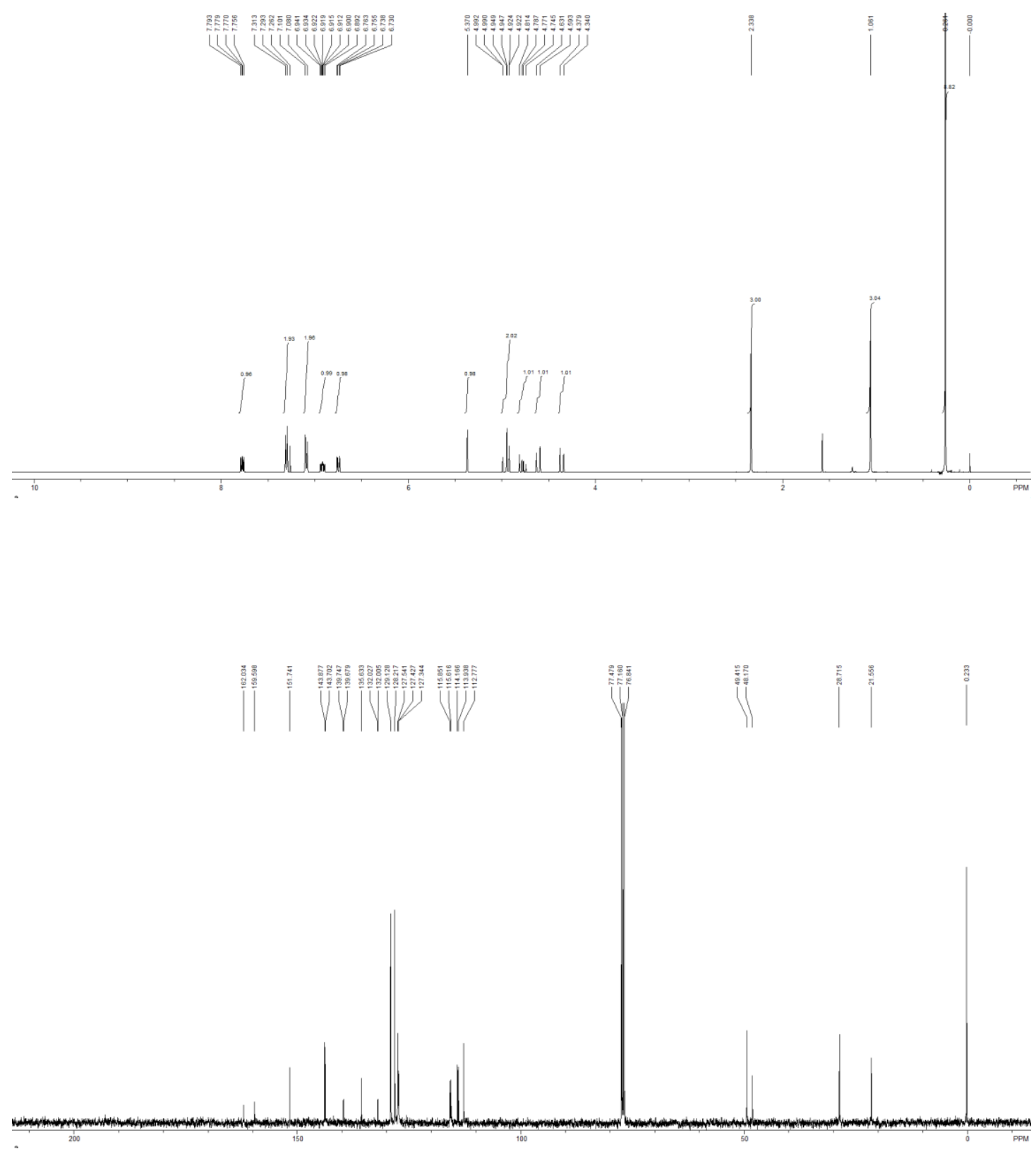


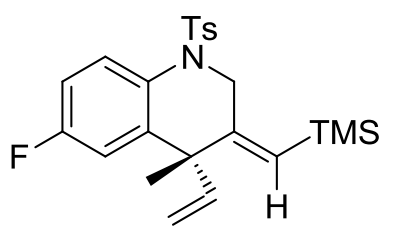

$4 e$

W

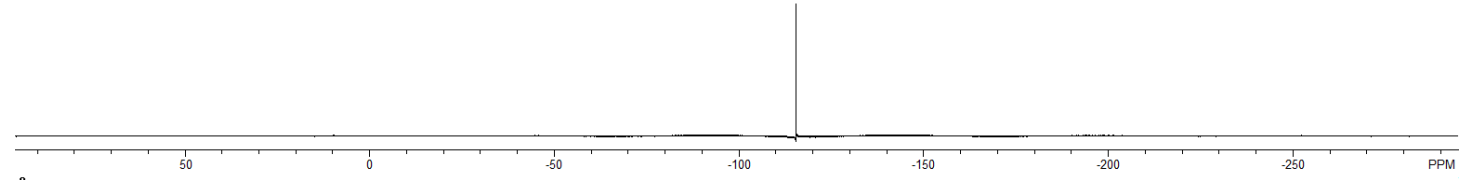


<smiles>C=C[C@]1(C)/C(=C/[Y5](C)(C)C)CN([As])c2ccc(Cl)cc21</smiles>

4f

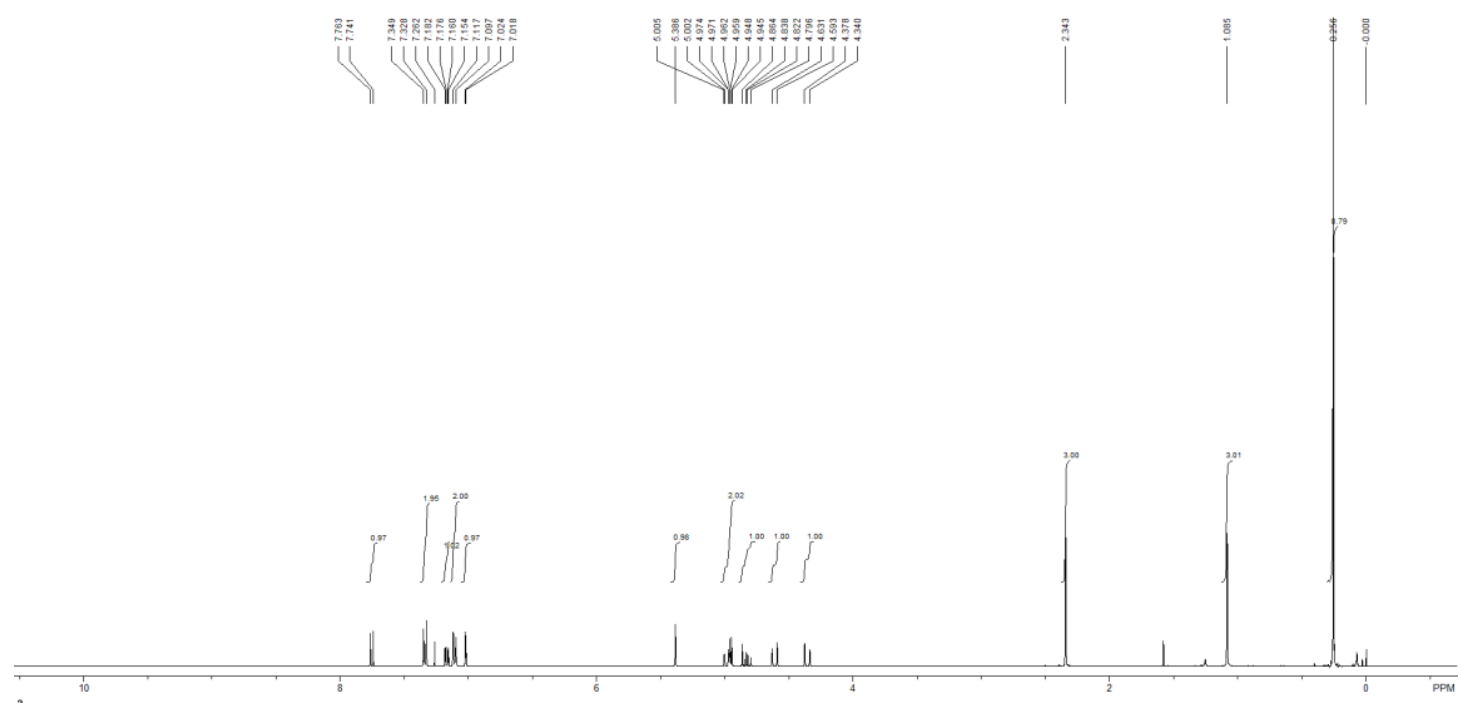

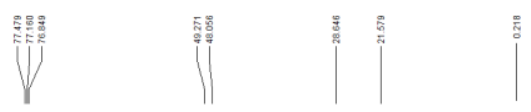

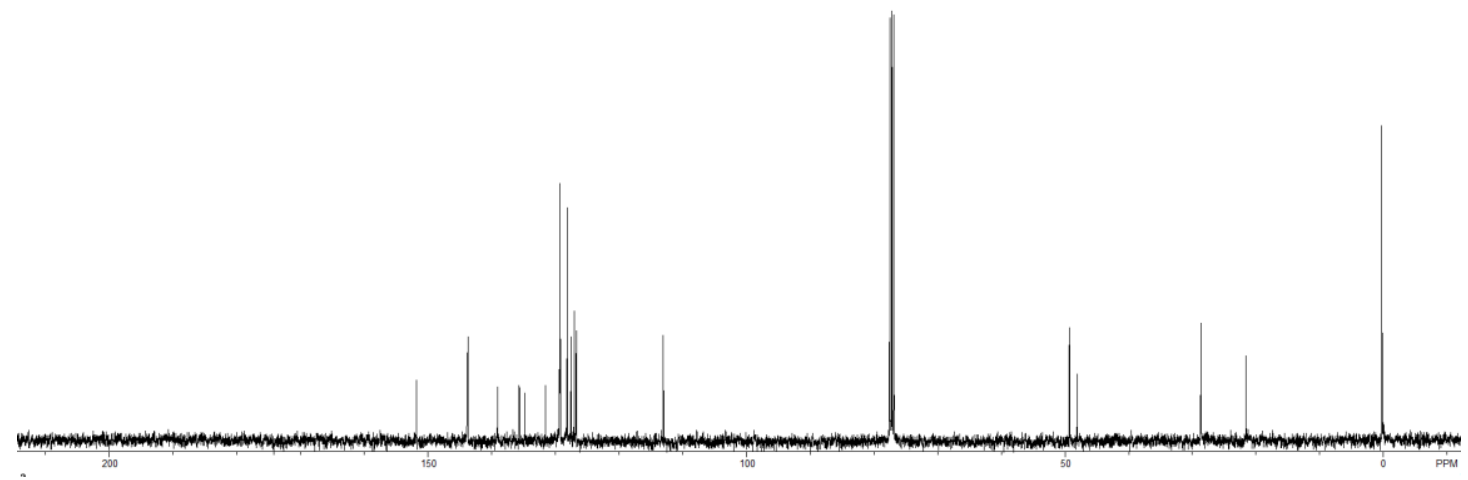


<smiles>C=C[C@]1(C)/C(=C/[Y15](=O)[O-])CN([As])c2ccc(Br)cc21</smiles>

$4 \mathrm{~g}$
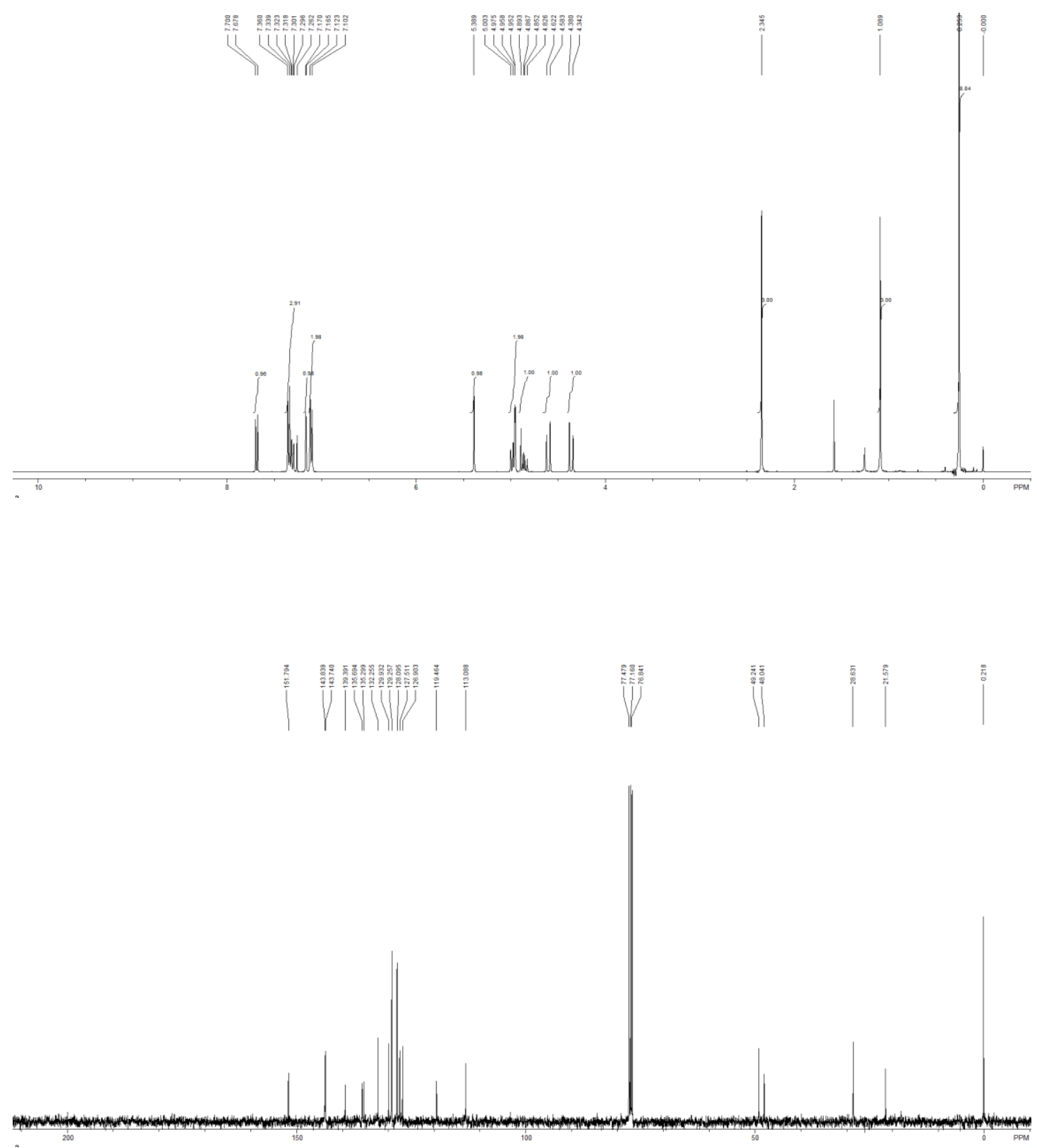
<smiles>C=C[C@]1(C)/C(=C/[Y15](=O)[O-])CN([As])c2ccc(C(F)(F)F)cc21</smiles>

$4 h$

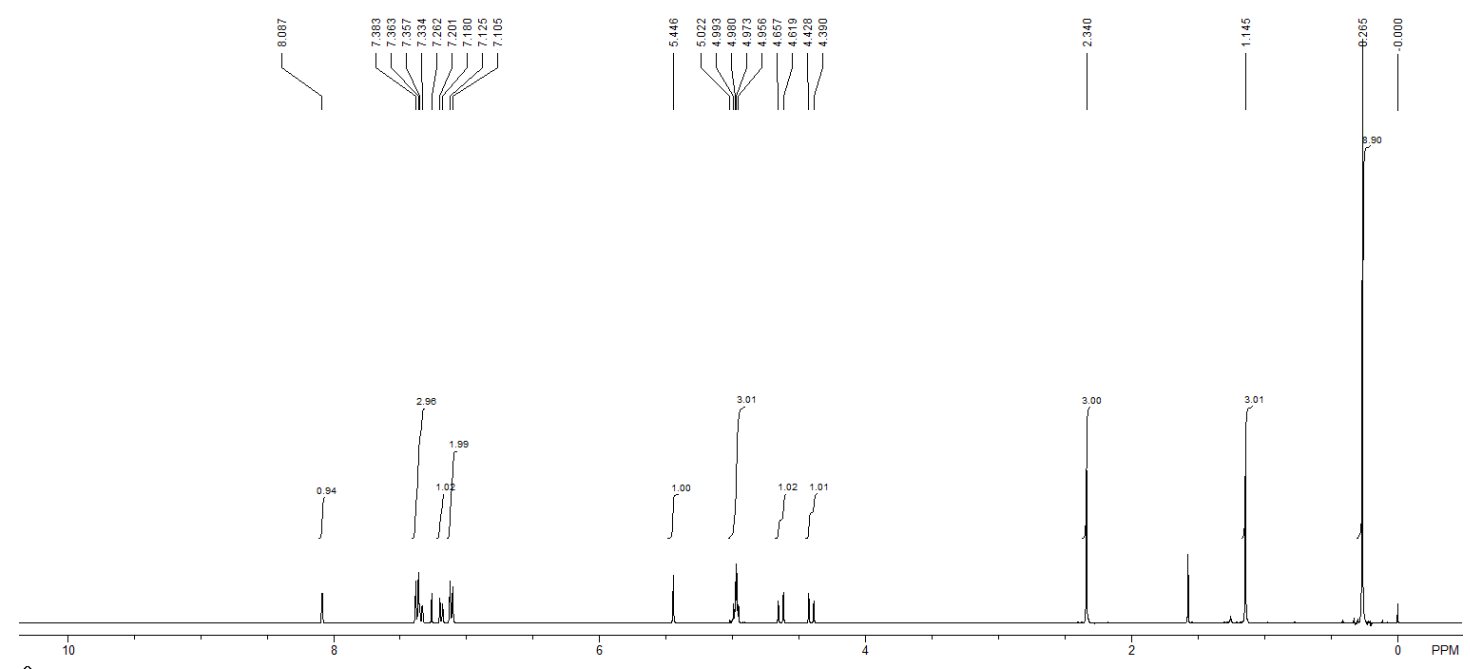

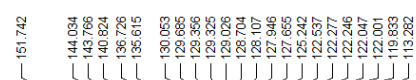

(1)

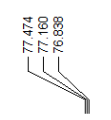

象
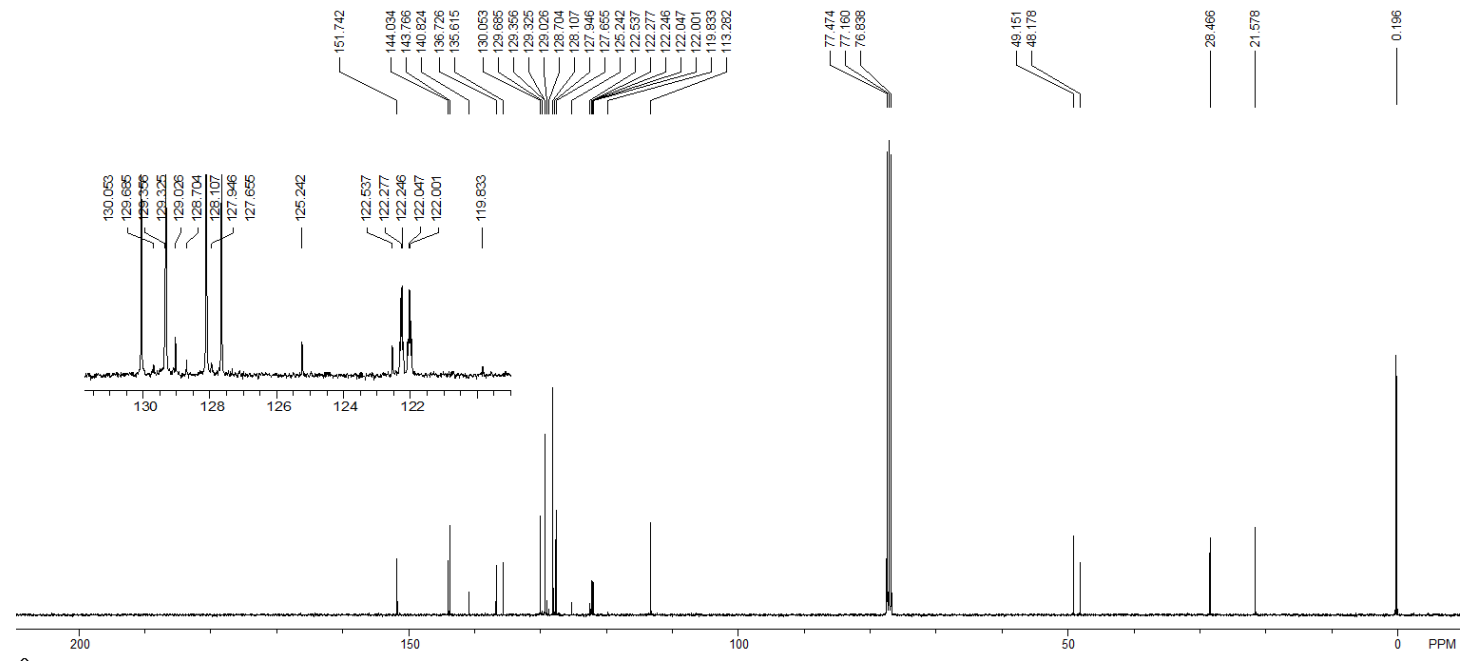


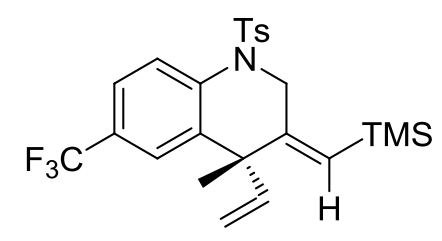

4h

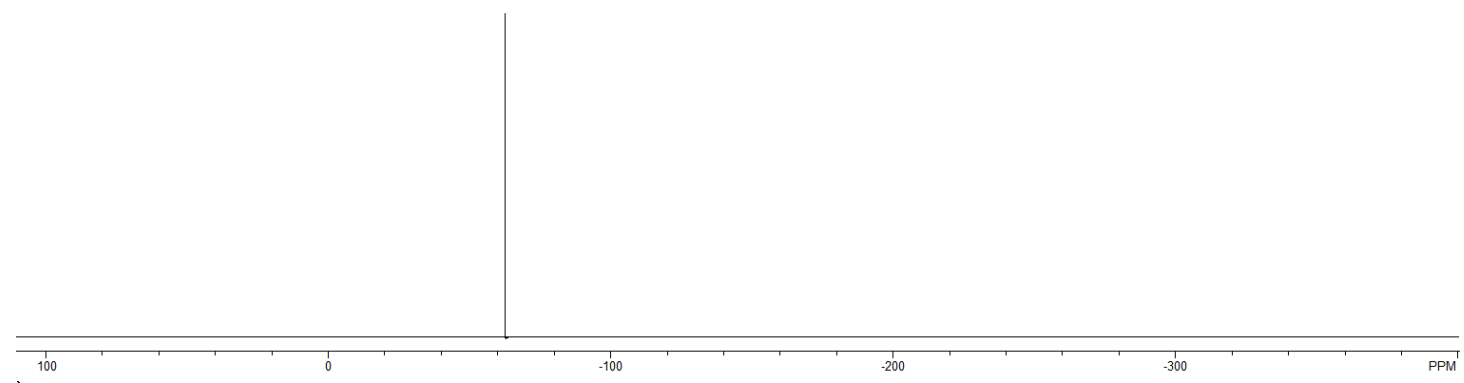


<smiles>C=C[C@]1(C)/C(=C/[13C](C)C)CN([As])c2ccccc21</smiles>

4i

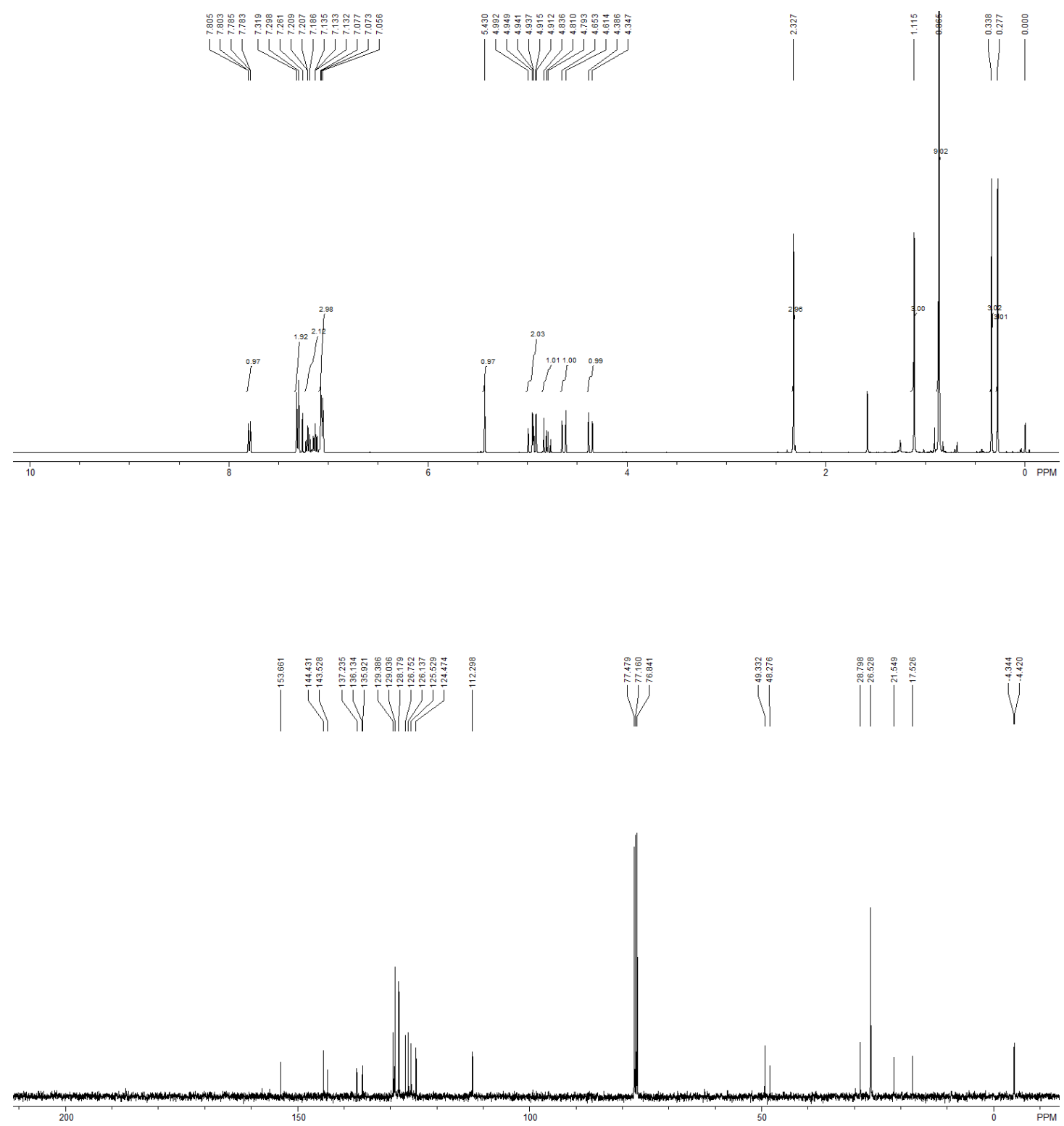


<smiles>C=C[C@@]1(C)/C(=C\C(C)(C)C)C[N]c2ccccc21</smiles>

4j

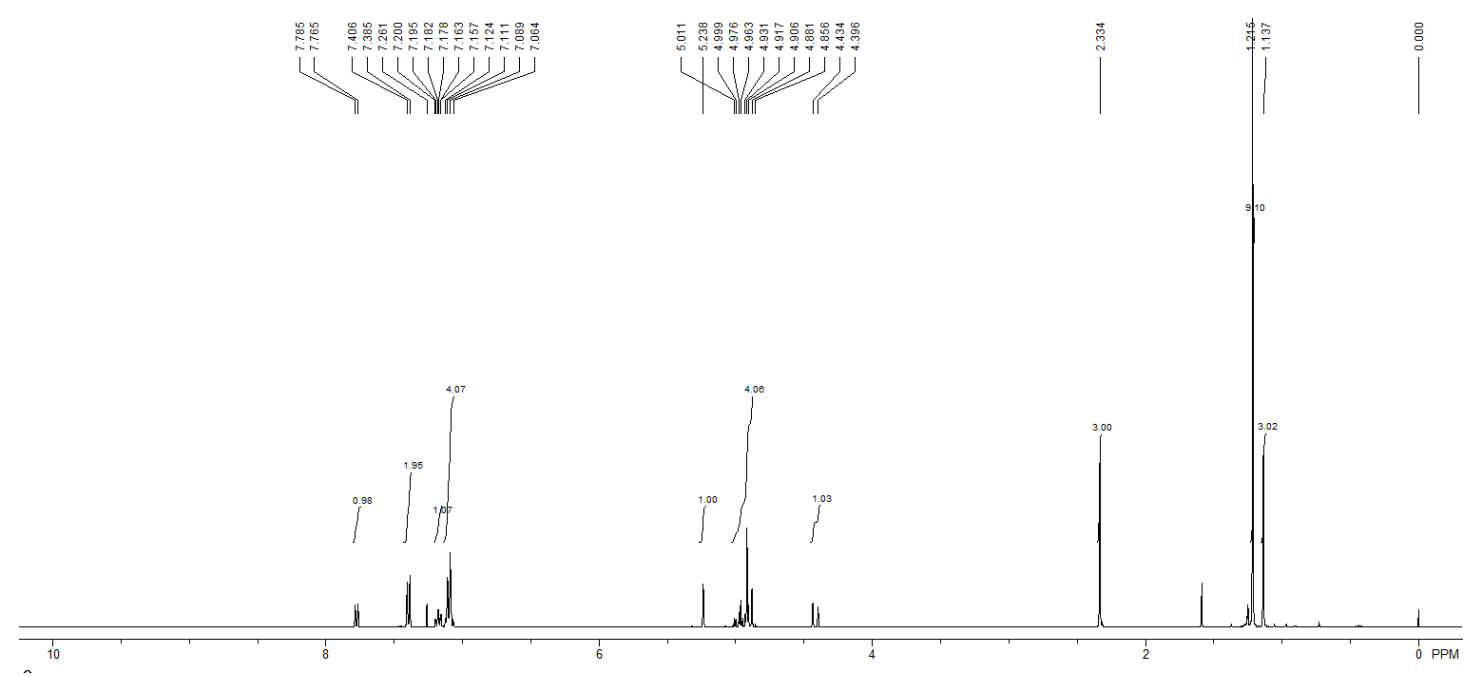

$\prod^{2}$
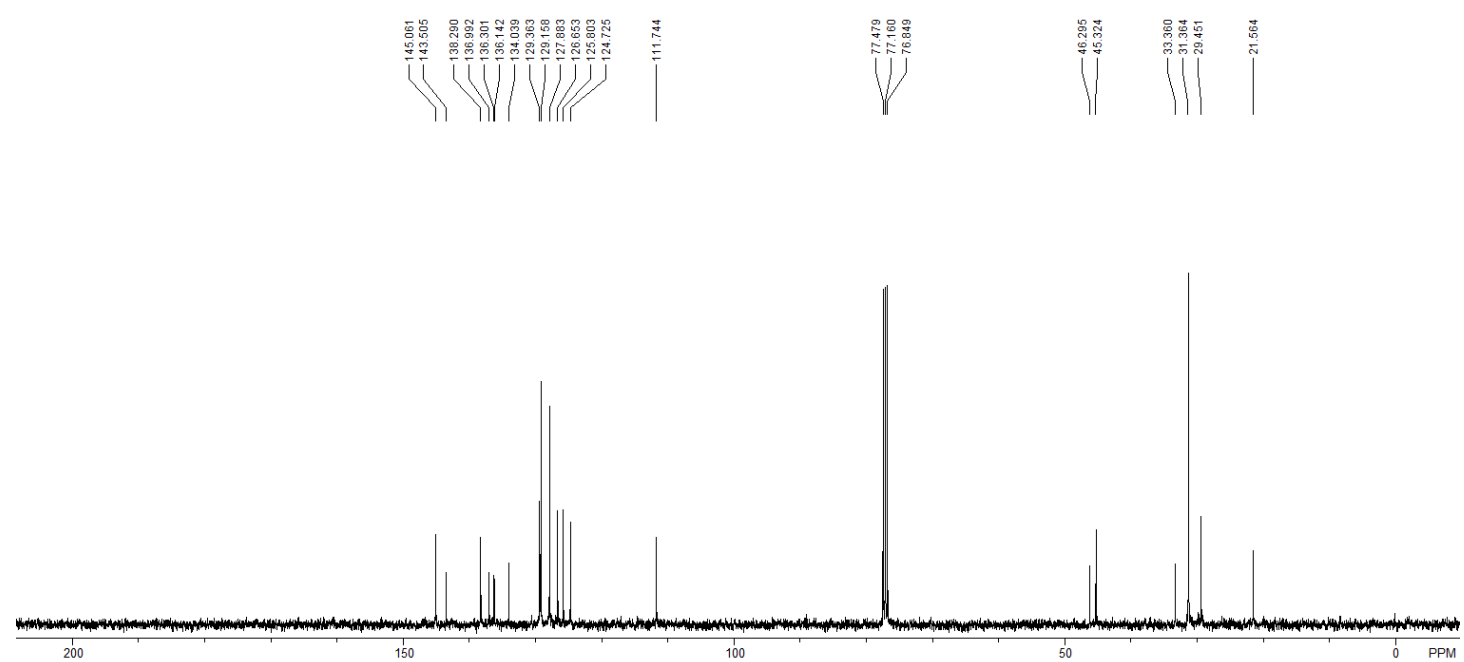


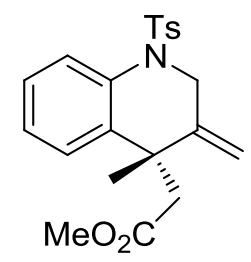

$5 a$

象哭

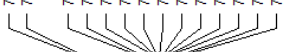

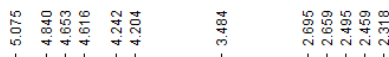

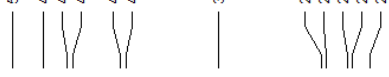
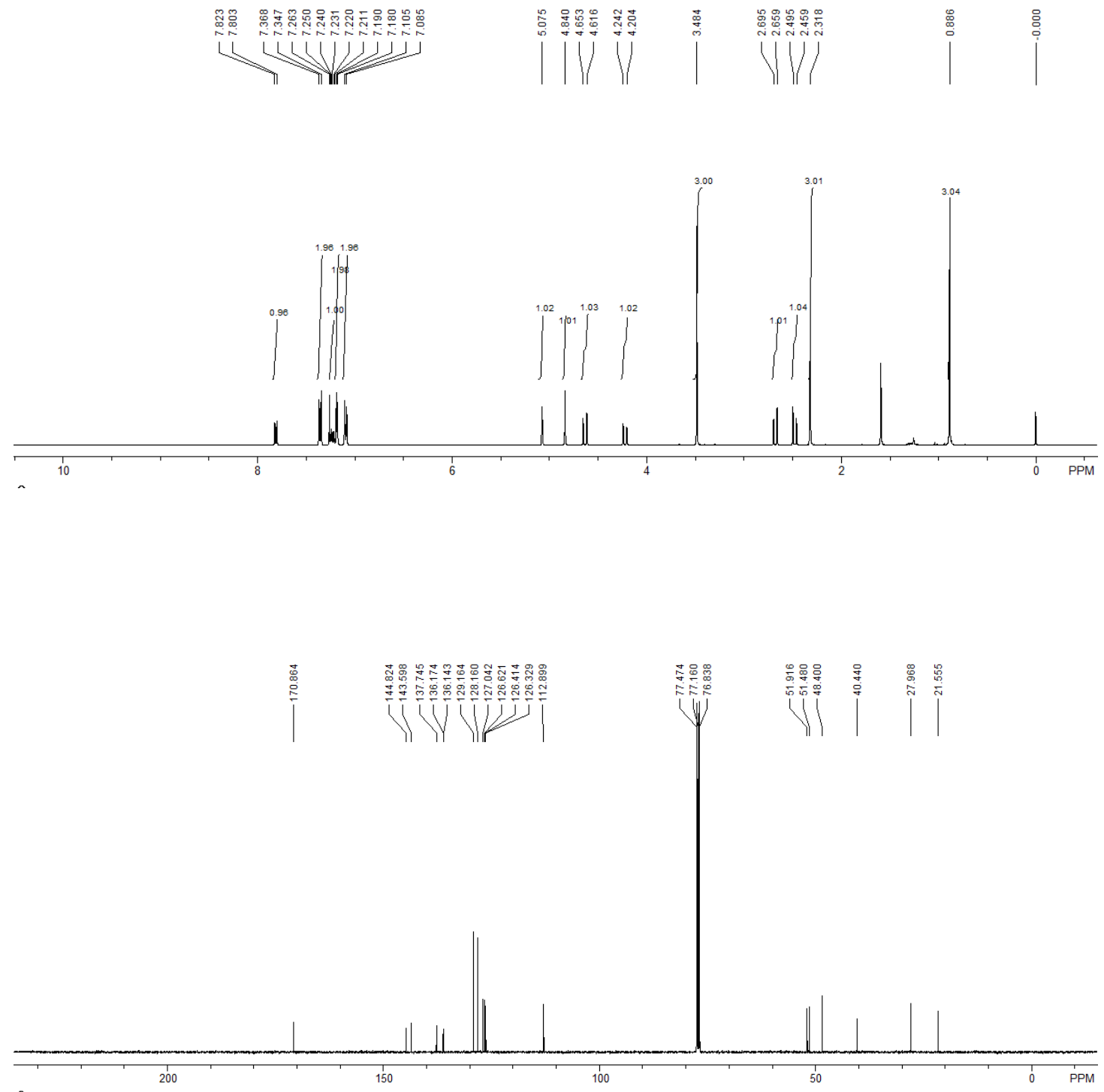


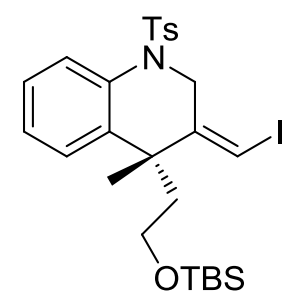

$6 a$
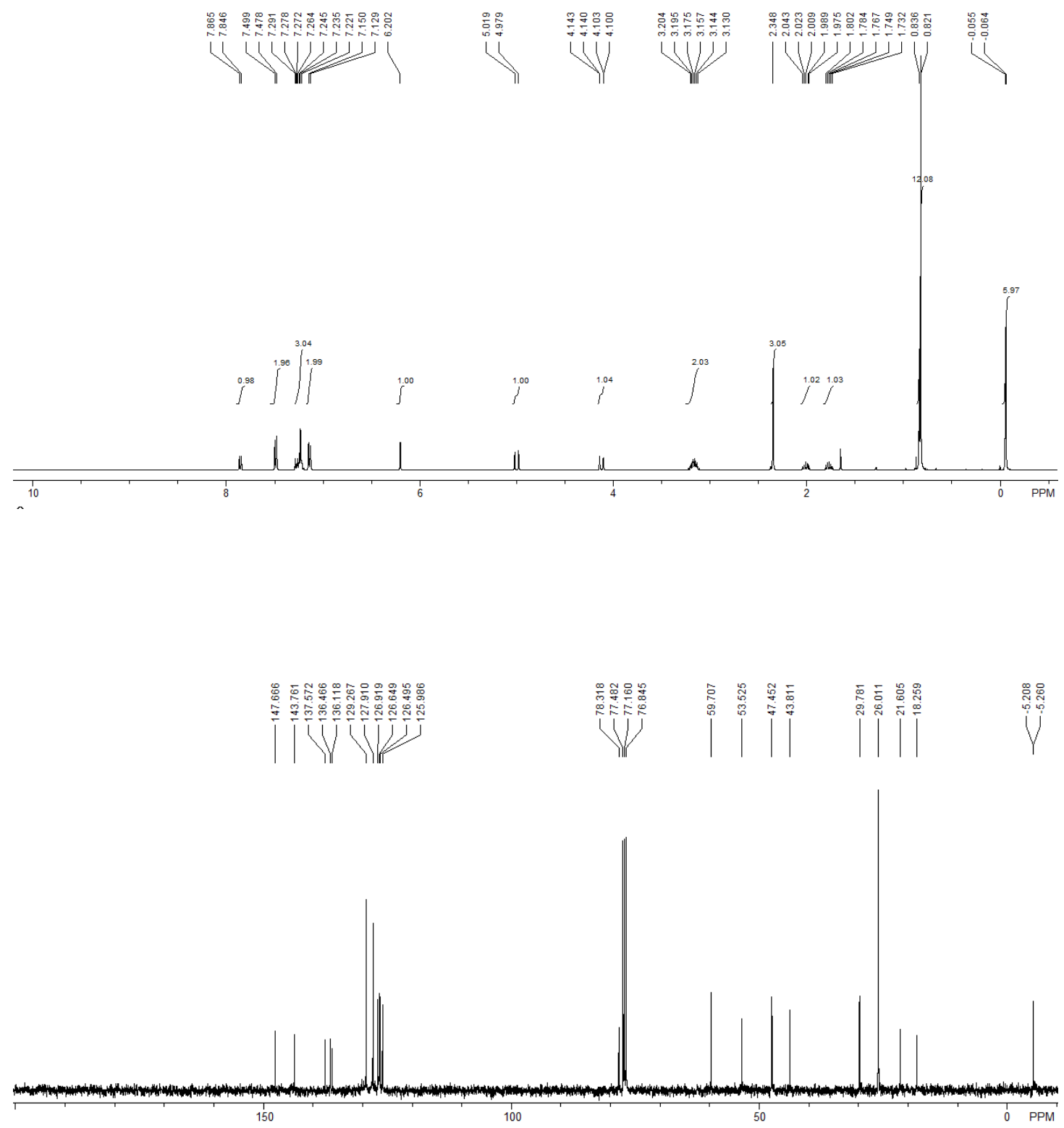


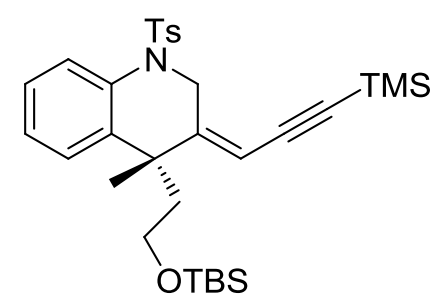

$7 a$

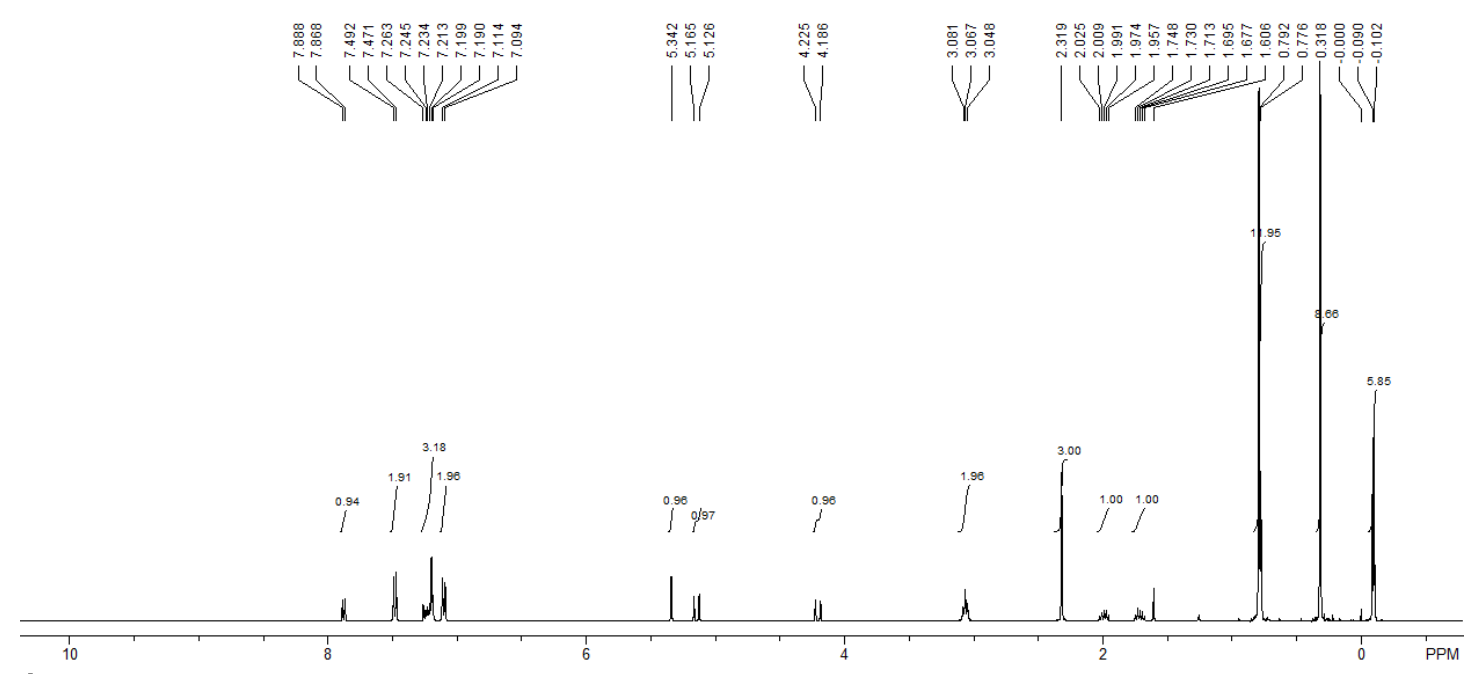

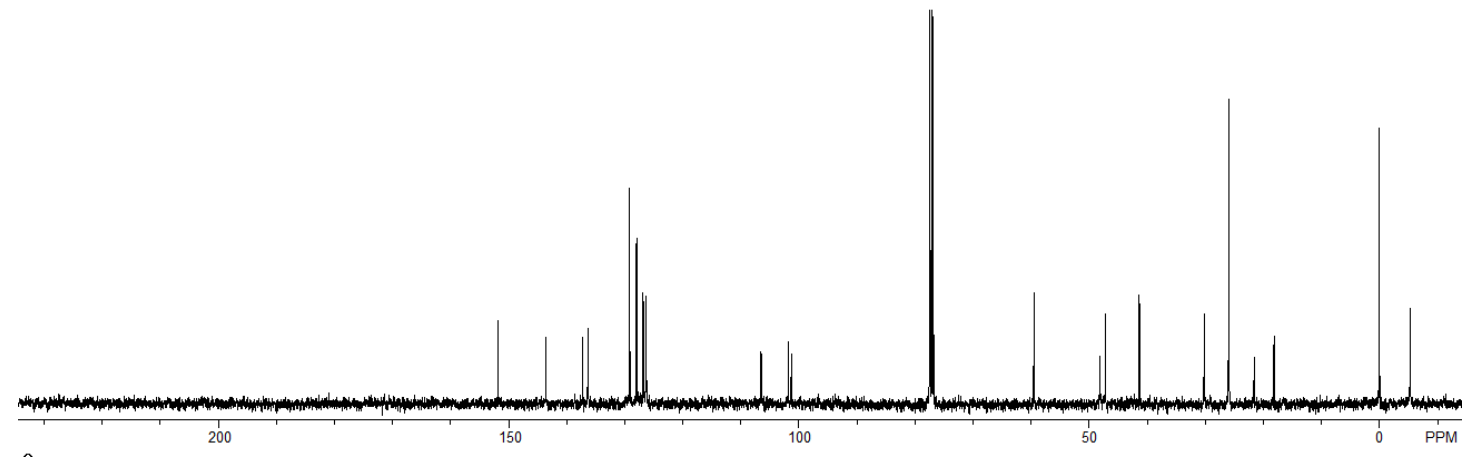


<smiles></smiles>

$8 a$
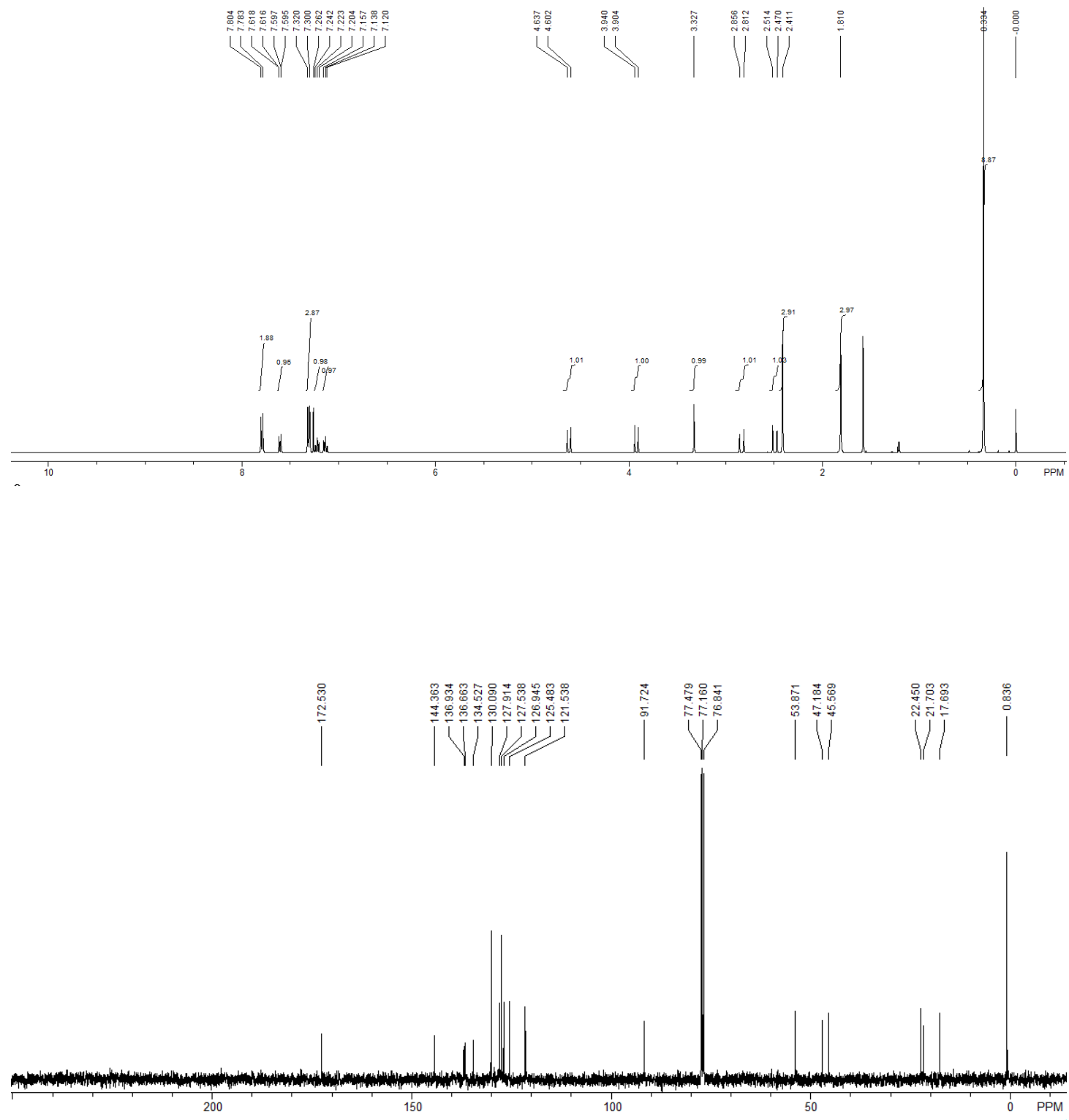

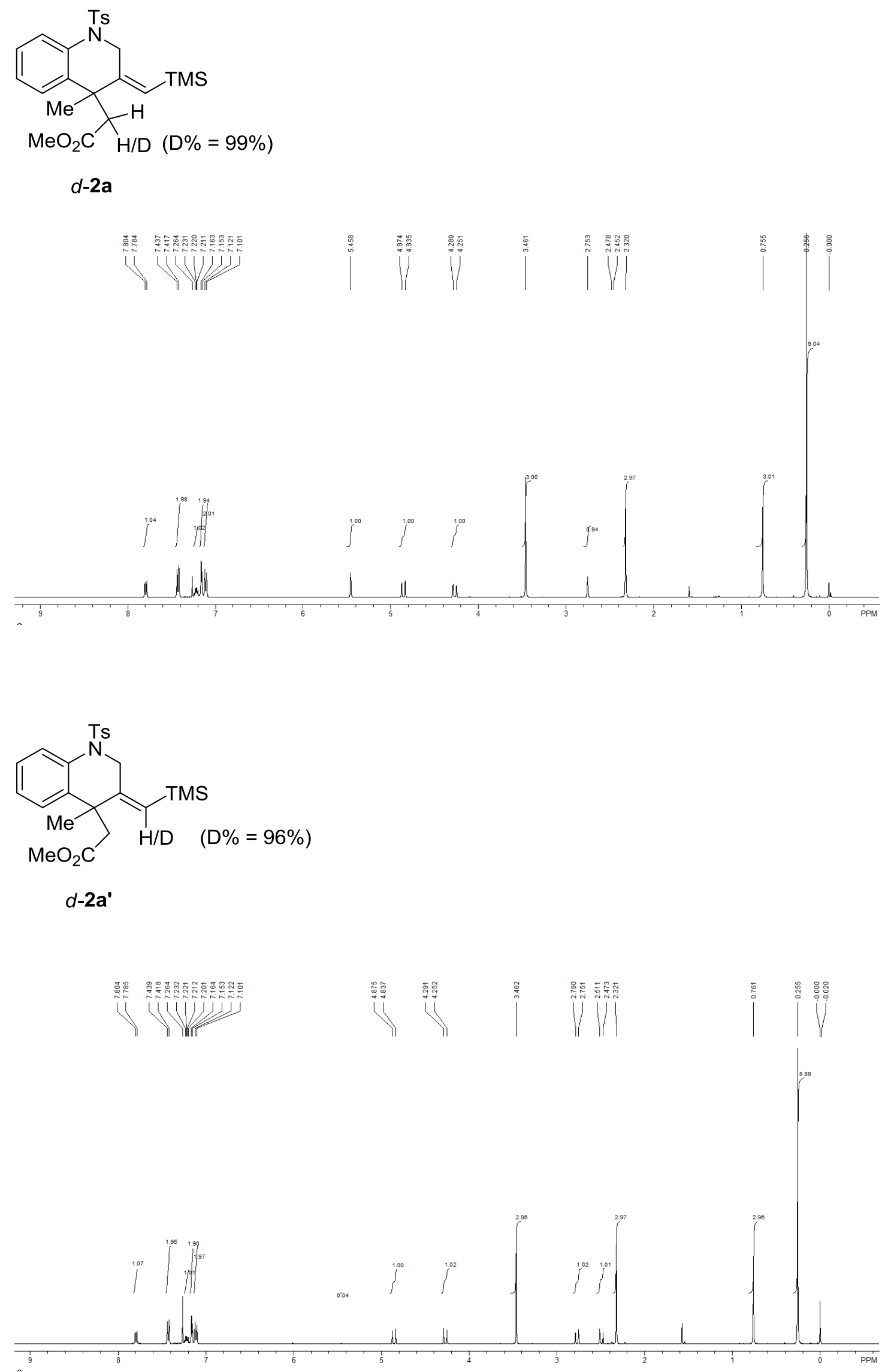


\section{HPLC analysis reports of chiral products}<smiles>CC(=O)OC[C@@]1(C)/C(=C/C(C)=S)CN([As])c2ccccc21</smiles>

2a
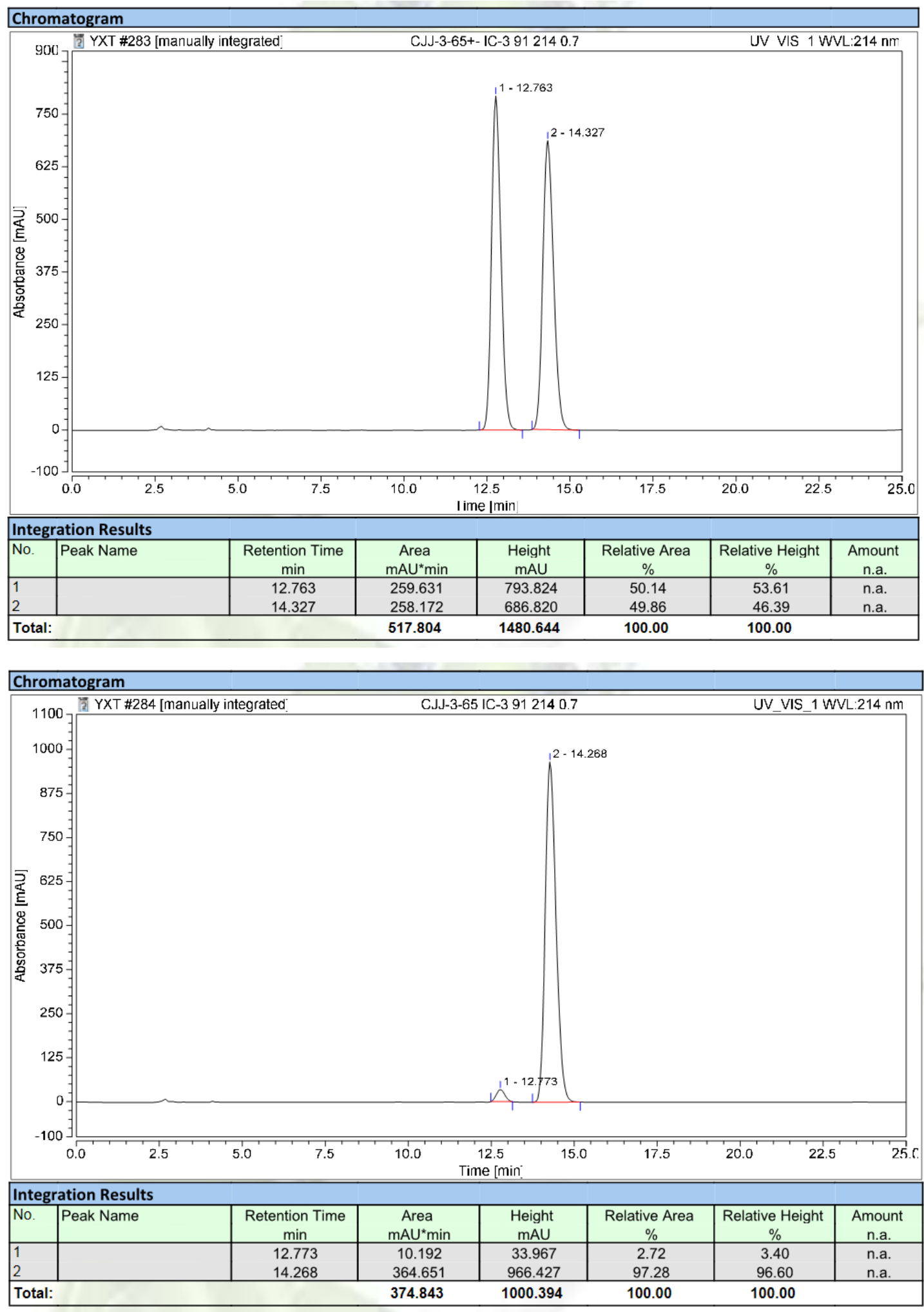
<smiles>CC(=O)/C=C1/CN([As])c2ccc(C)cc2[C@]1(C)CC(C)=O</smiles>

2b

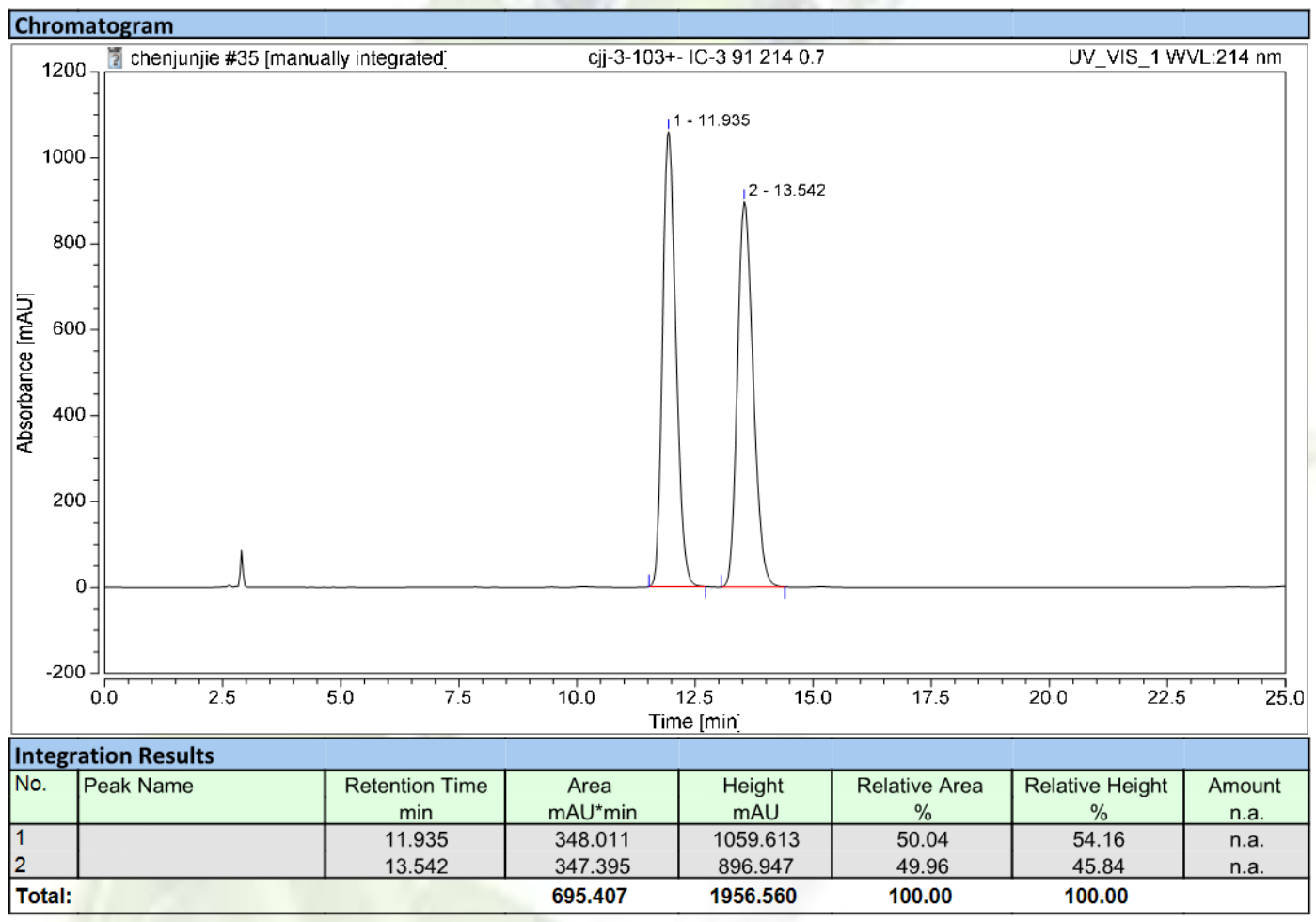

\section{Chromatogram}

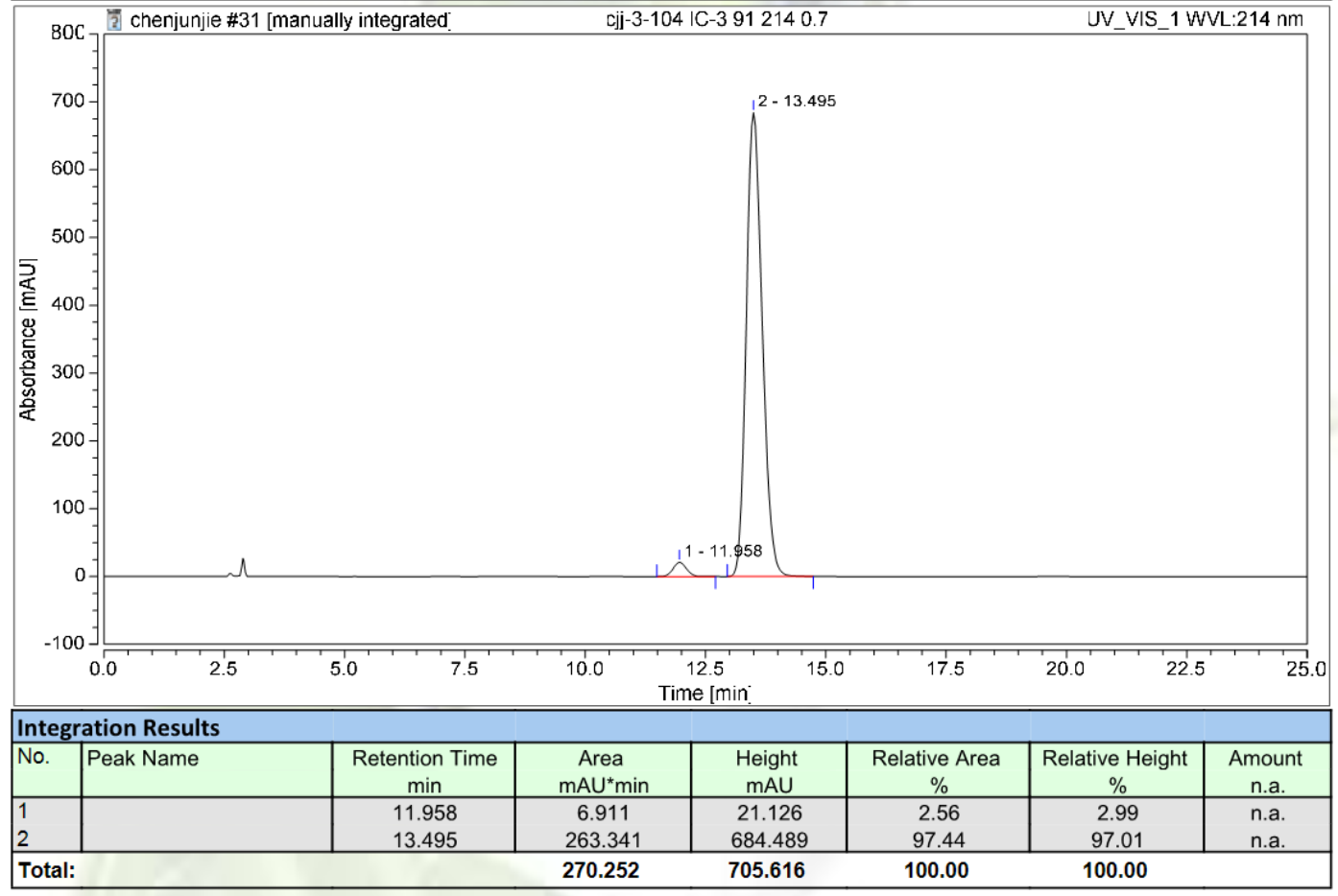



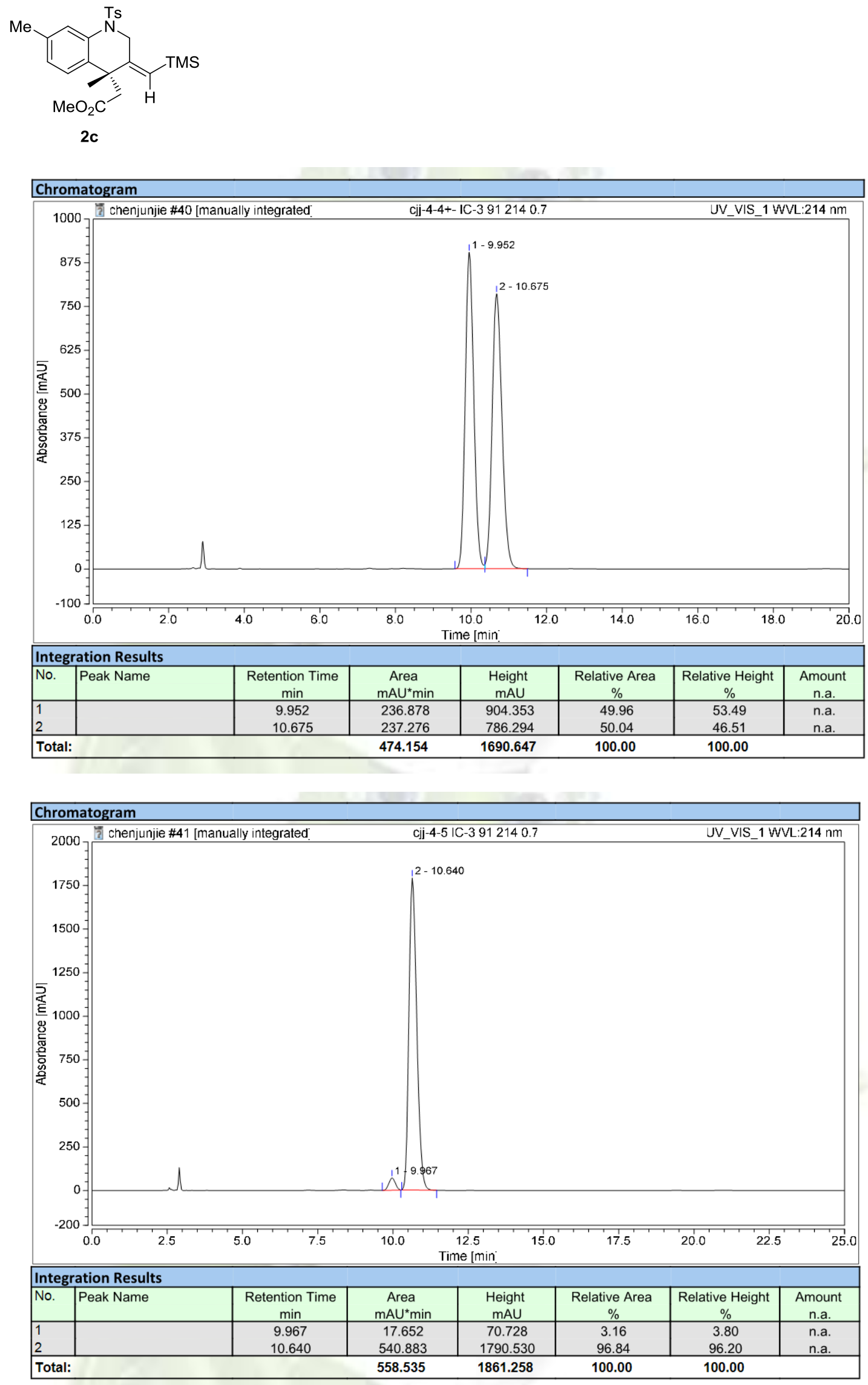
<smiles>COc1ccc2c(c1)[C@@]1(CC(C)=O)C[C+]2NC/C1=C\C(C)=O</smiles>
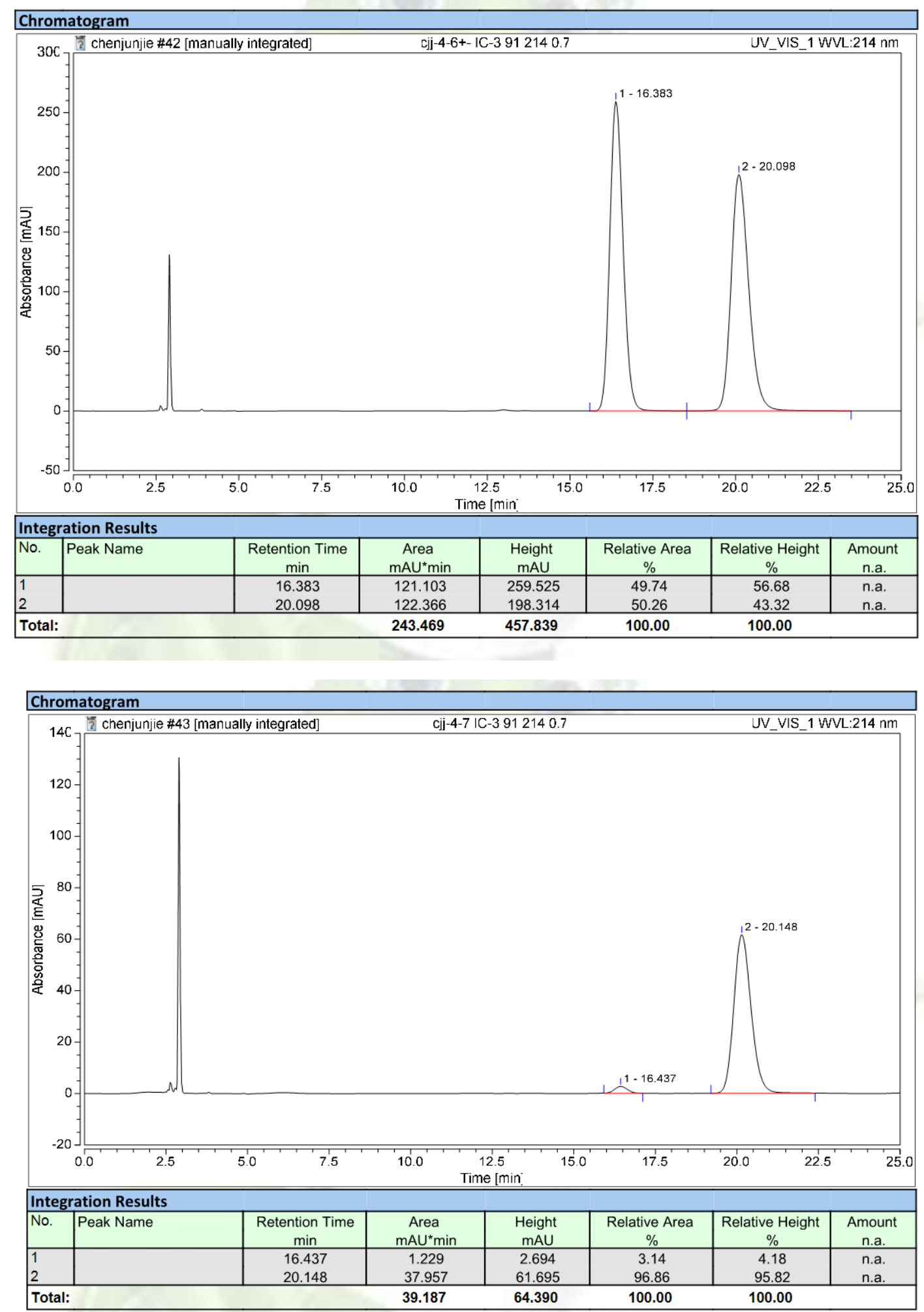
<smiles>CC(=O)CC1(C)/C(=C(/C)S(C)(=O)=O)C[N]c2ccc(F)cc21</smiles>

$2 e$
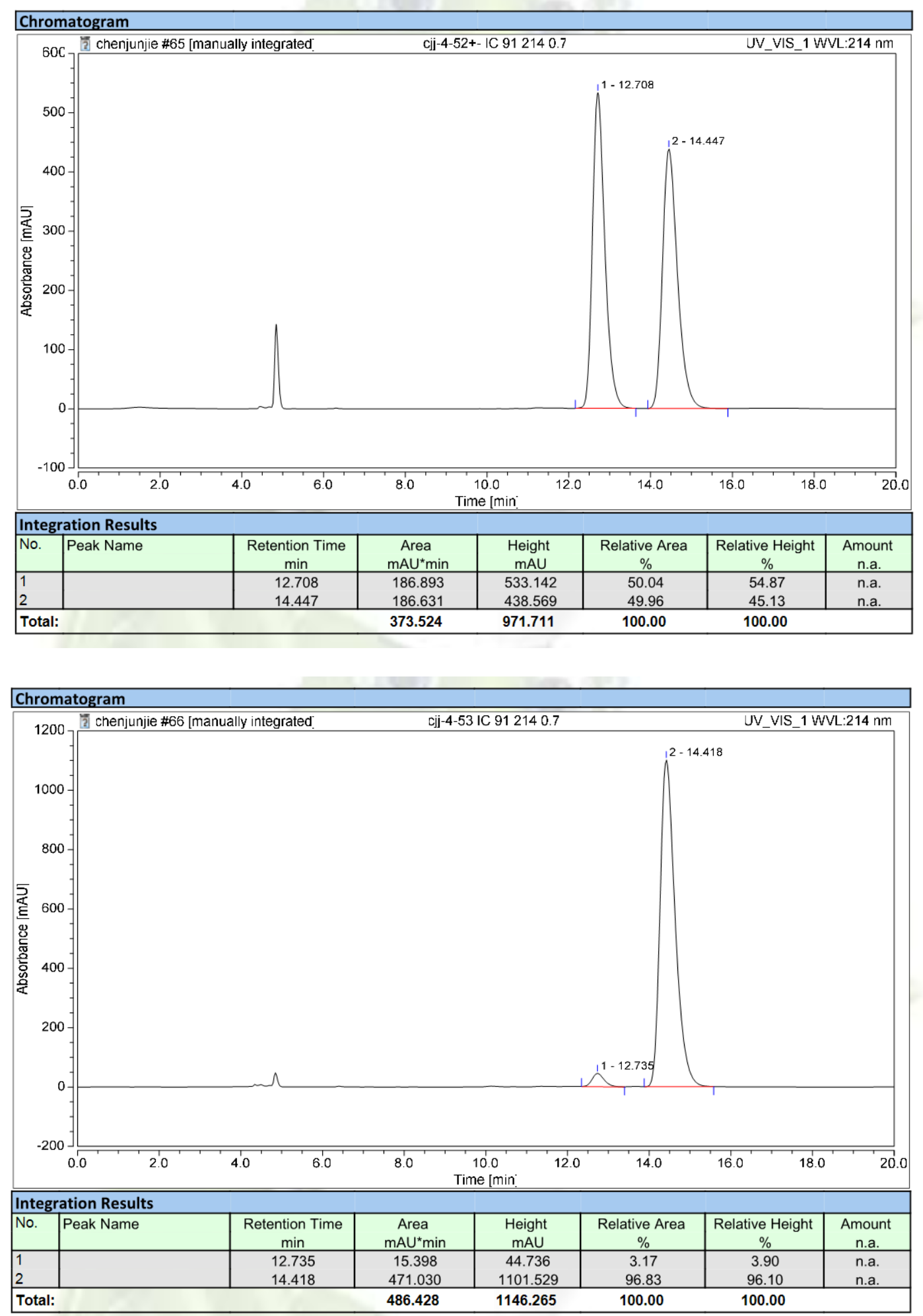
<smiles>CCC1(C(C)=O)C(=C[N+](=O)[O-])CNc2ccc(Cl)cc21</smiles>

$2 f$

\section{Chromatogram}

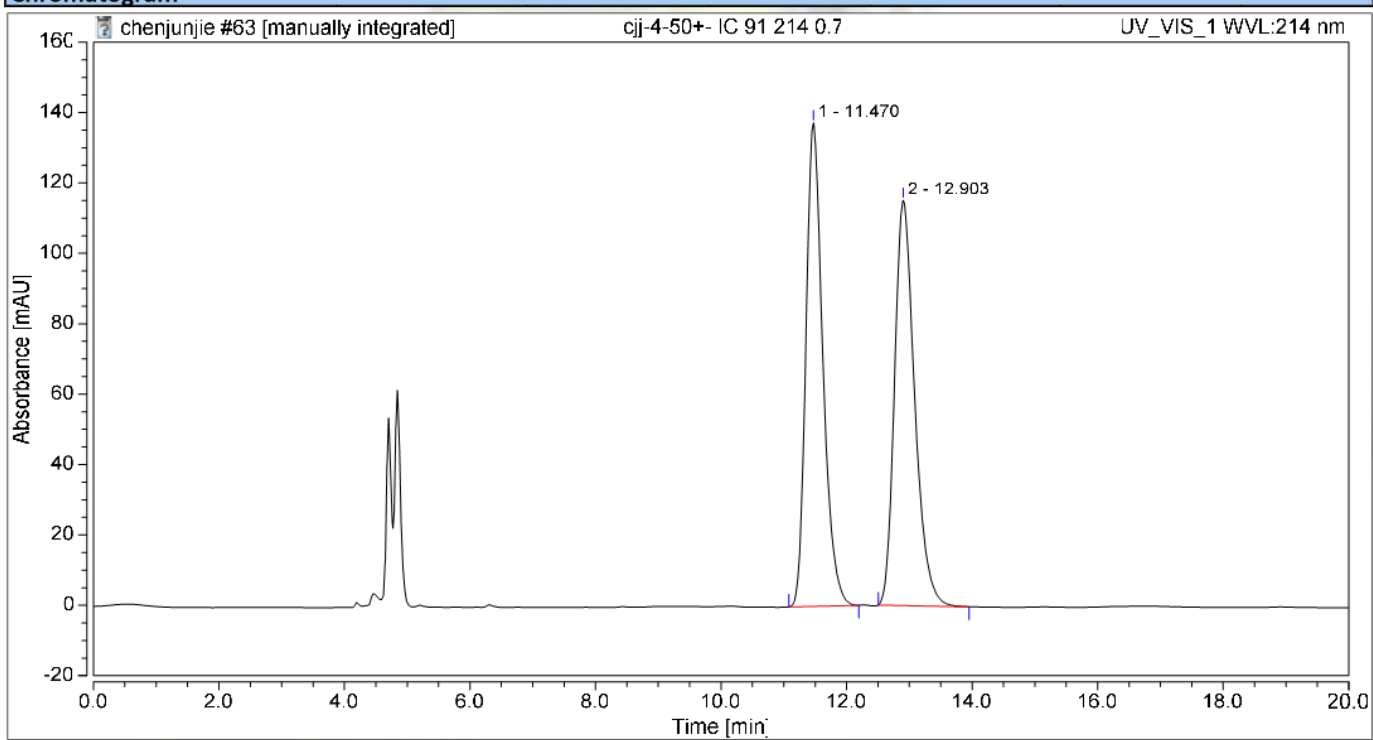

\begin{tabular}{|c|c|c|c|c|c|c|c|}
\hline \multicolumn{8}{|c|}{ Integration Results } \\
\hline No. & Peak Name & $\begin{array}{c}\text { Retention Time } \\
\text { min }\end{array}$ & $\begin{array}{c}\text { Area } \\
m A U^{*} \text { min }\end{array}$ & $\begin{array}{c}\text { Height } \\
\text { mAU }\end{array}$ & $\begin{array}{c}\text { Relative Area } \\
\%\end{array}$ & $\begin{array}{c}\text { Relative Height } \\
\%\end{array}$ & $\begin{array}{c}\text { Amount } \\
\text { n.a. }\end{array}$ \\
\hline 1 & & 11.470 & 42.921 & 137.353 & 50.22 & 54.42 & n.a. \\
\hline 2 & & 12.903 & 42.543 & 115.043 & 49.78 & 45.58 & n.a. \\
\hline \multicolumn{3}{|c|}{ Total: } & 85.464 & 252.397 & 100.00 & 100.00 & \\
\hline
\end{tabular}

\section{Chromatogram}

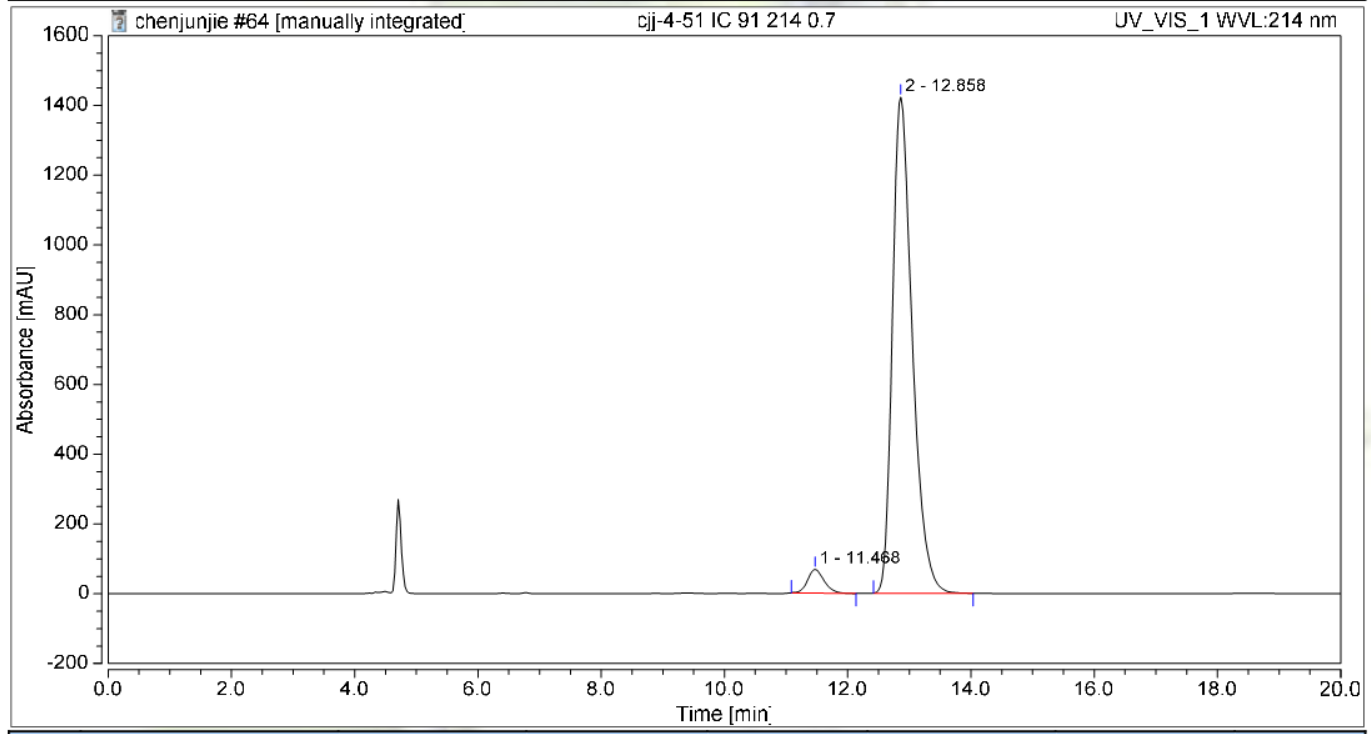

\begin{tabular}{|l|l|c|c|c|c|c|c|}
\hline Integration Results \\
\hline No. & Peak Name & $\begin{array}{c}\text { Retention Time } \\
\text { min }\end{array}$ & $\begin{array}{c}\text { Area } \\
\mathrm{mAU} \text { (min }\end{array}$ & $\begin{array}{c}\text { Height } \\
\mathrm{mAU}\end{array}$ & $\begin{array}{c}\text { Relative Area } \\
\%\end{array}$ & $\begin{array}{c}\text { Relative Height } \\
\%\end{array}$ & $\begin{array}{c}\text { Amount } \\
\text { n.a. }\end{array}$ \\
\hline 1 & & 11.468 & 21.215 & 68.086 & 3.85 & 4.57 & n.a. \\
2 & 12.858 & 530.505 & 1422.441 & 96.15 & 95.43 & n.a. \\
\hline
\end{tabular}


<smiles>CCC1(CC(C)=O)/C(=C\C(C)=O)C[NH2+]c2ccc(Br)cc21</smiles>

$2 \mathrm{~g}$
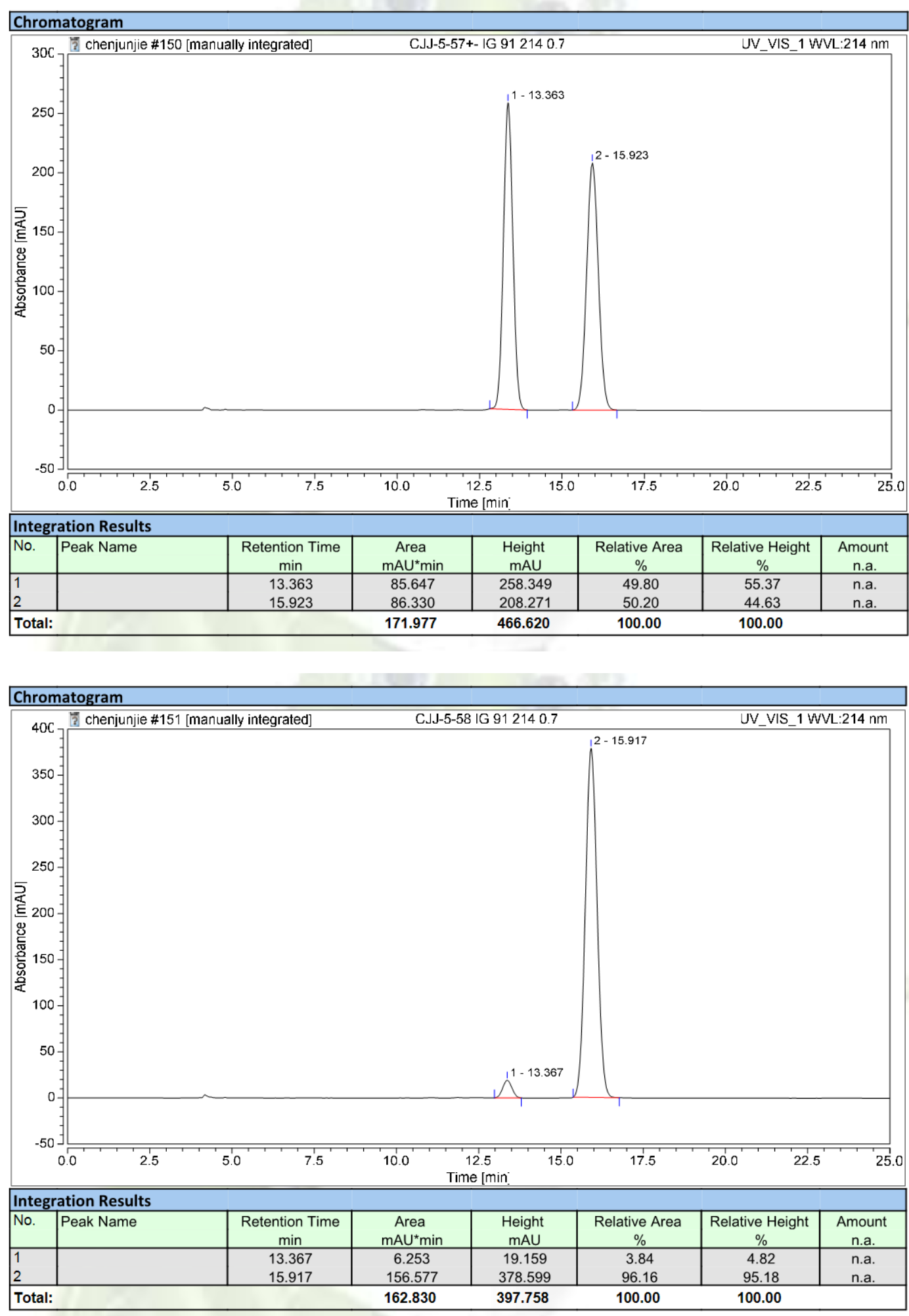
<smiles>CCC1(C)/C(=C/C(C)=O)CNc2ccc(C(F)(F)F)cc21</smiles>

$2 \mathrm{~h}$
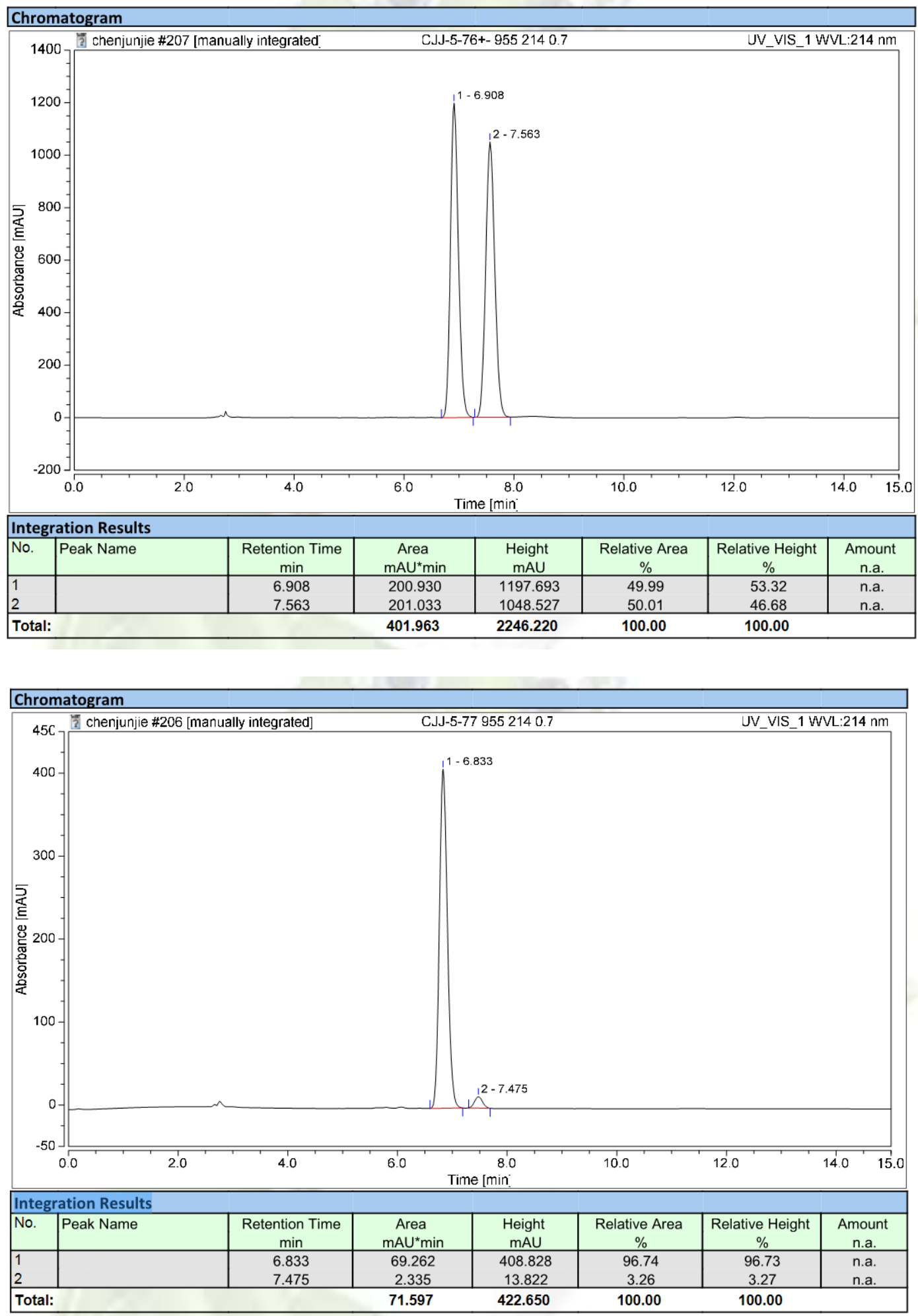
<smiles>CCC1(CC(C)(C)C)C(=CC(C)=O)C[NH2+]c2ccc(-c3ccccc3)cc21</smiles>

2i

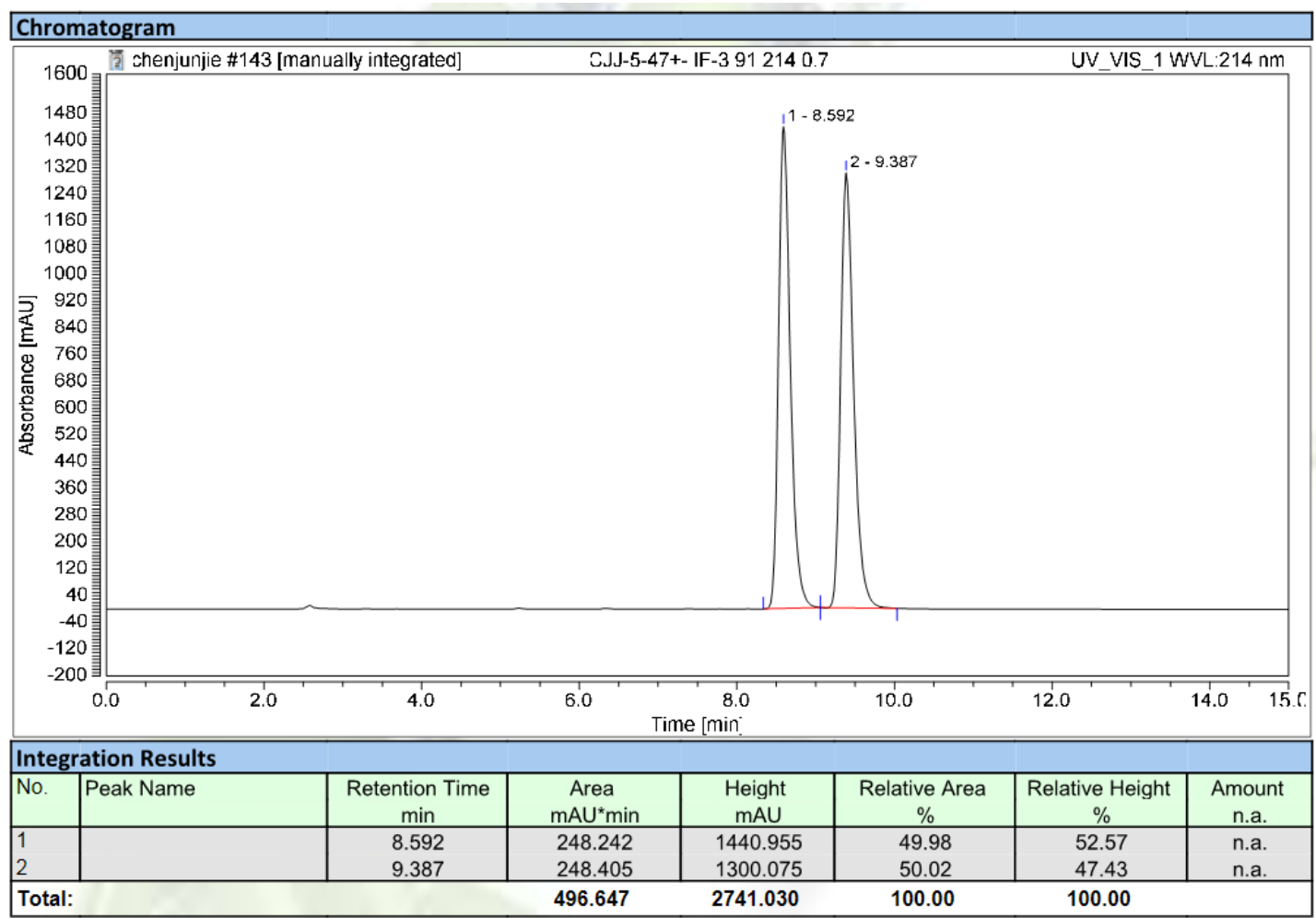

\section{Chromatogram}

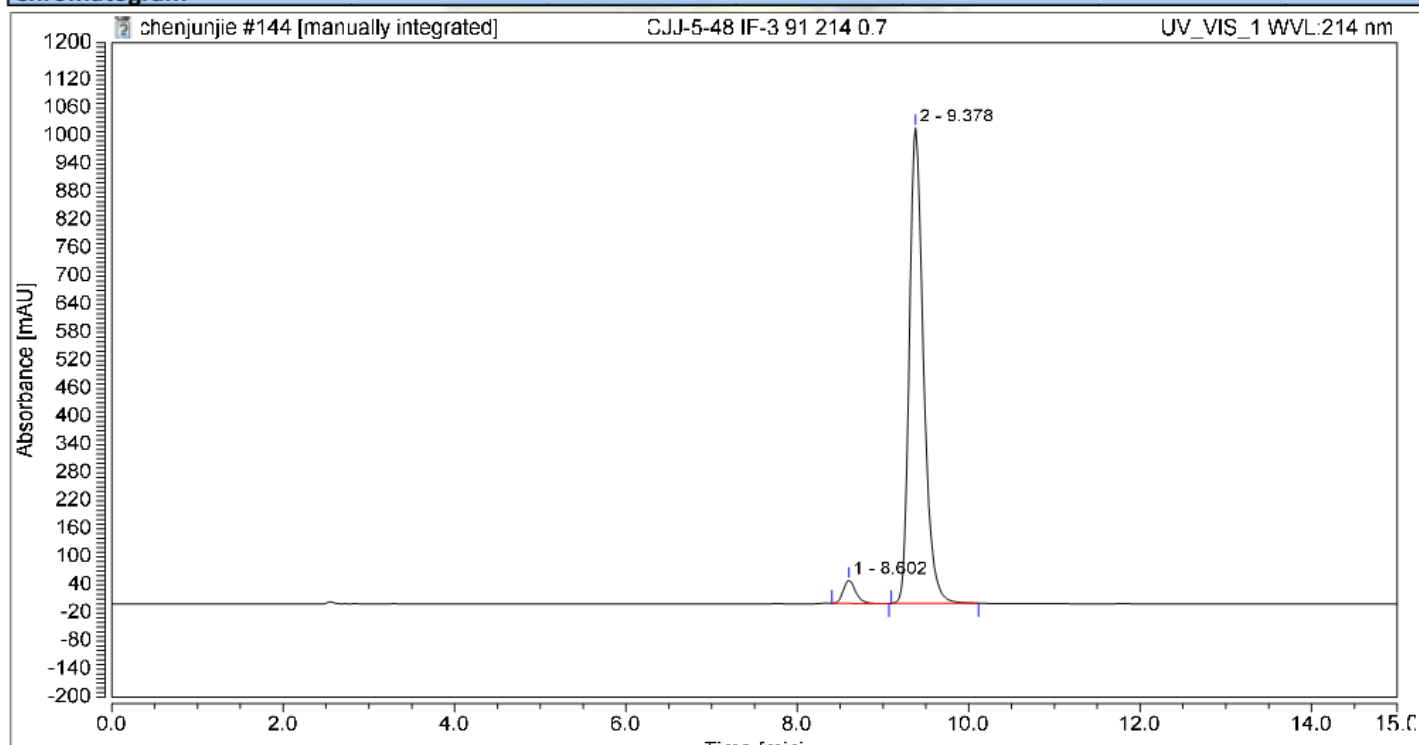

\begin{tabular}{|c|c|c|c|c|c|c|c|}
\hline \multicolumn{8}{|c|}{ Time [min] } \\
\hline \multicolumn{8}{|c|}{ Integration Results } \\
\hline No. & Peak Name & $\begin{array}{l}\text { Retention Time } \\
\text { min }\end{array}$ & $\begin{array}{c}\text { Area } \\
\mathrm{mAU}^{*} \mathrm{~min}\end{array}$ & $\begin{array}{c}\text { Height } \\
\text { mAU }\end{array}$ & $\begin{array}{c}\text { Relative Area } \\
\%\end{array}$ & $\begin{array}{c}\text { Relative Height } \\
\%\end{array}$ & $\begin{array}{c}\text { Amount } \\
\text { n.a. }\end{array}$ \\
\hline 1 & & 8.602 & 8.183 & 49.304 & 4.03 & 4.62 & n.a. \\
\hline 2 & & 9.378 & 194.777 & 1016.943 & 95.97 & 95.38 & n.a. \\
\hline \multicolumn{3}{|c|}{ Total: } & 202.959 & 1066.247 & 100.00 & 100.00 & \\
\hline
\end{tabular}


DMPS

2j

\section{Chromatogram}
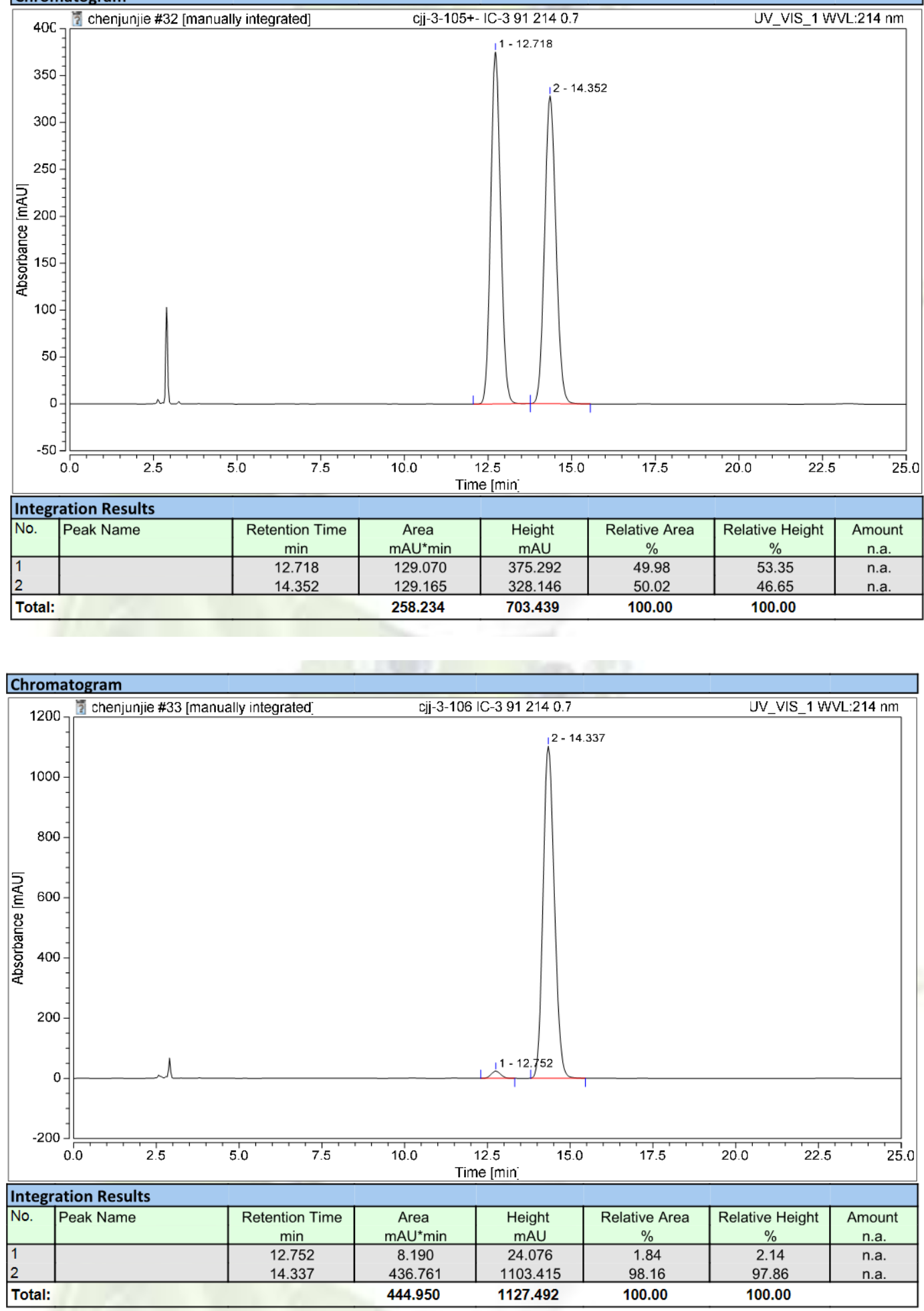


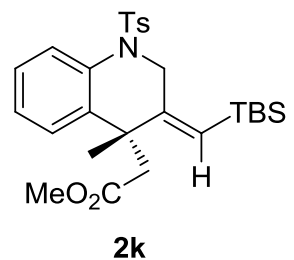

\section{Chromatogram}

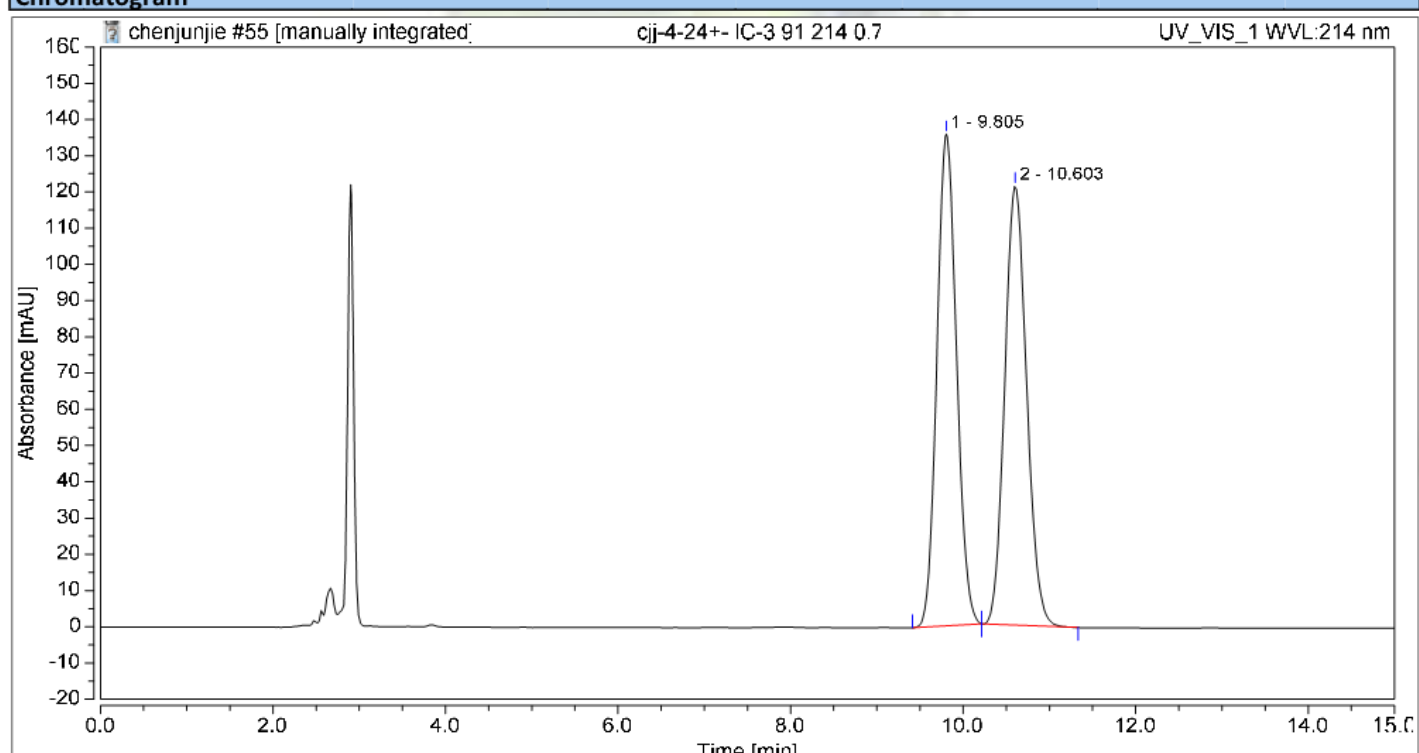

\begin{tabular}{|c|c|c|c|c|c|c|c|}
\hline \multicolumn{8}{|c|}{ Integration Results } \\
\hline No. & Peak Name & $\begin{array}{c}\text { Retention Time } \\
\text { min }\end{array}$ & $\begin{array}{c}\text { Area } \\
m A U^{*} \min \end{array}$ & $\begin{array}{c}\text { Height } \\
\text { mAU }\end{array}$ & $\begin{array}{c}\text { Relative Area } \\
\%\end{array}$ & $\begin{array}{c}\text { Relative Height } \\
\%\end{array}$ & $\begin{array}{c}\text { Amount } \\
\text { n.a. }\end{array}$ \\
\hline 1 & & 9.805 & 35.710 & 135.772 & 50.05 & 52.82 & n.a. \\
\hline 2 & & 10.603 & 35.641 & 121.259 & 49.95 & 47.18 & n.a. \\
\hline \multicolumn{3}{|c|}{ Total: } & 71.351 & 257.030 & 100.00 & 100.00 & \\
\hline
\end{tabular}

\section{Chromatogram}

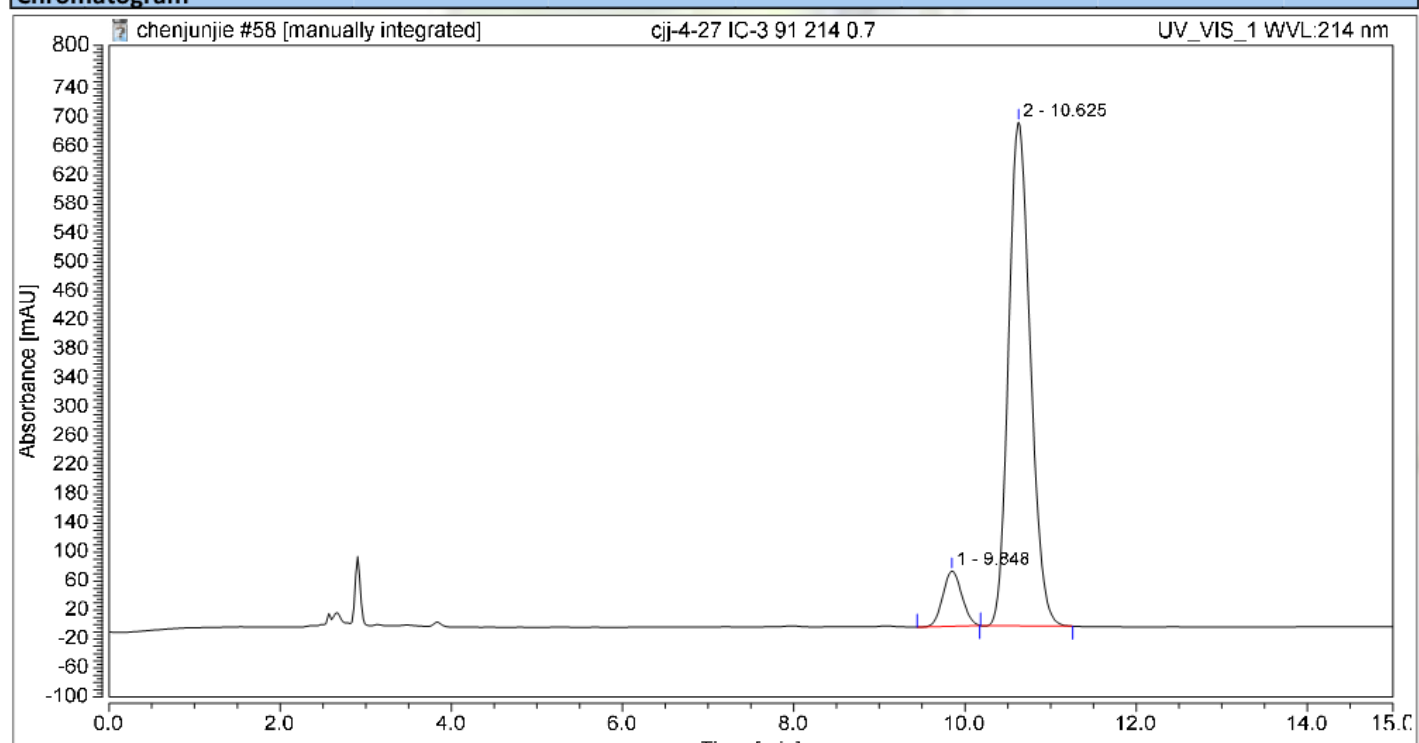

\begin{tabular}{|l|l|c|c|c|c|c|c|}
\hline \multicolumn{1}{|l|}{ Integration Results } \\
\hline No. & Peak Name & $\begin{array}{c}\text { Retention Time } \\
\text { min }\end{array}$ & $\begin{array}{c}\text { Area } \\
\text { mAU*min }\end{array}$ & $\begin{array}{c}\text { Height } \\
\text { mAU }\end{array}$ & $\begin{array}{c}\text { Relative Area } \\
\%\end{array}$ & $\begin{array}{c}\text { Relative Height } \\
\%\end{array}$ & $\begin{array}{c}\text { Amount } \\
\text { n.a. }\end{array}$ \\
\hline 1 & & 9.848 & 19.528 & 76.105 & 8.68 & 9.86 & n.a. \\
2 & 10.625 & 205.494 & 695.459 & 91.32 & 90.14 & n.a. \\
\hline
\end{tabular}




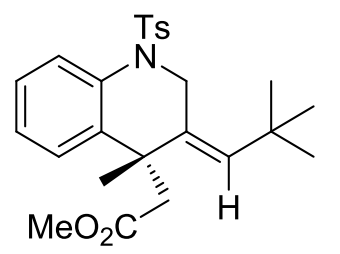

2I

\section{Chromatogram}

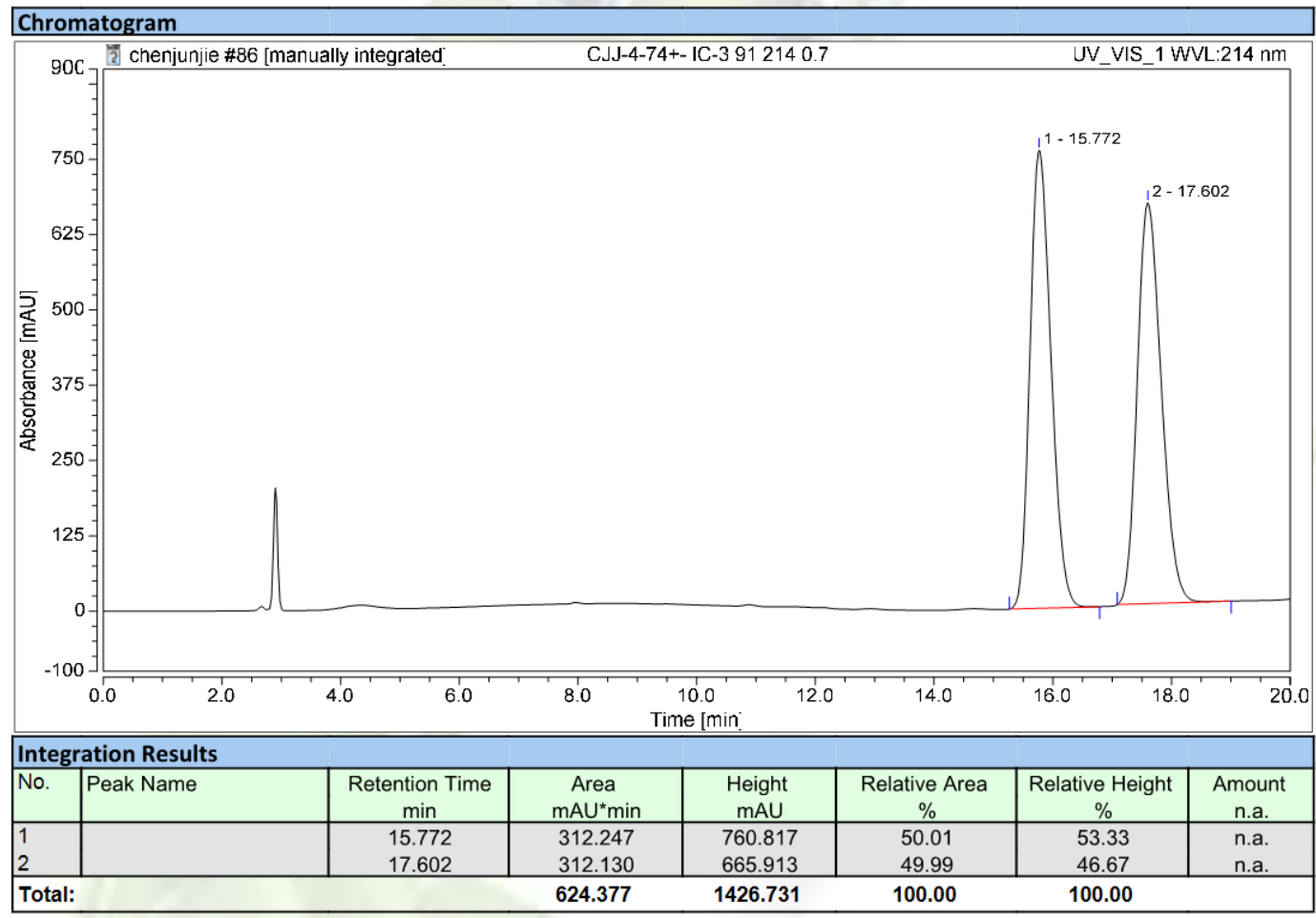

\section{Chromatogram}

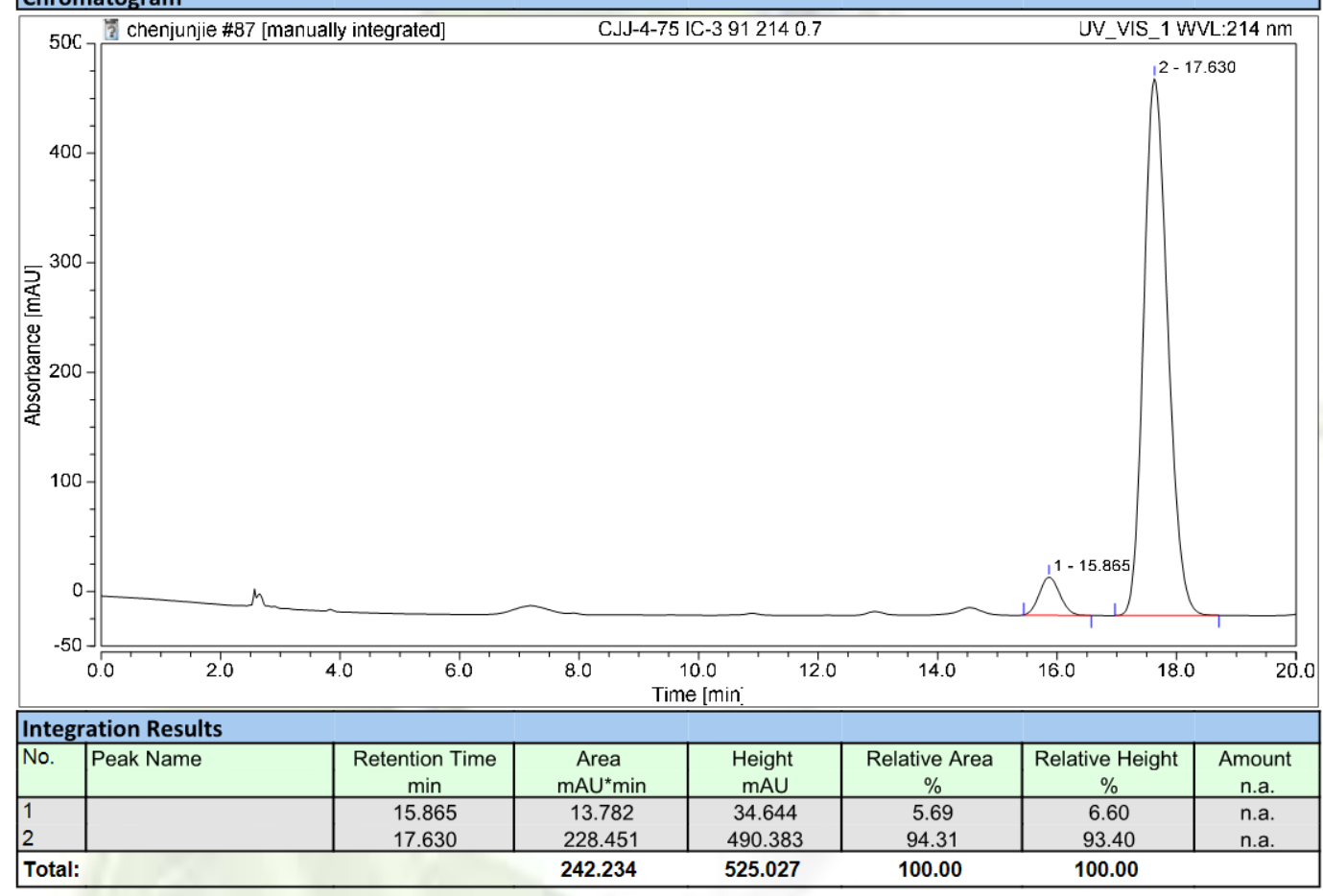




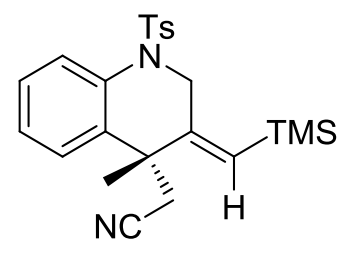

$2 m$
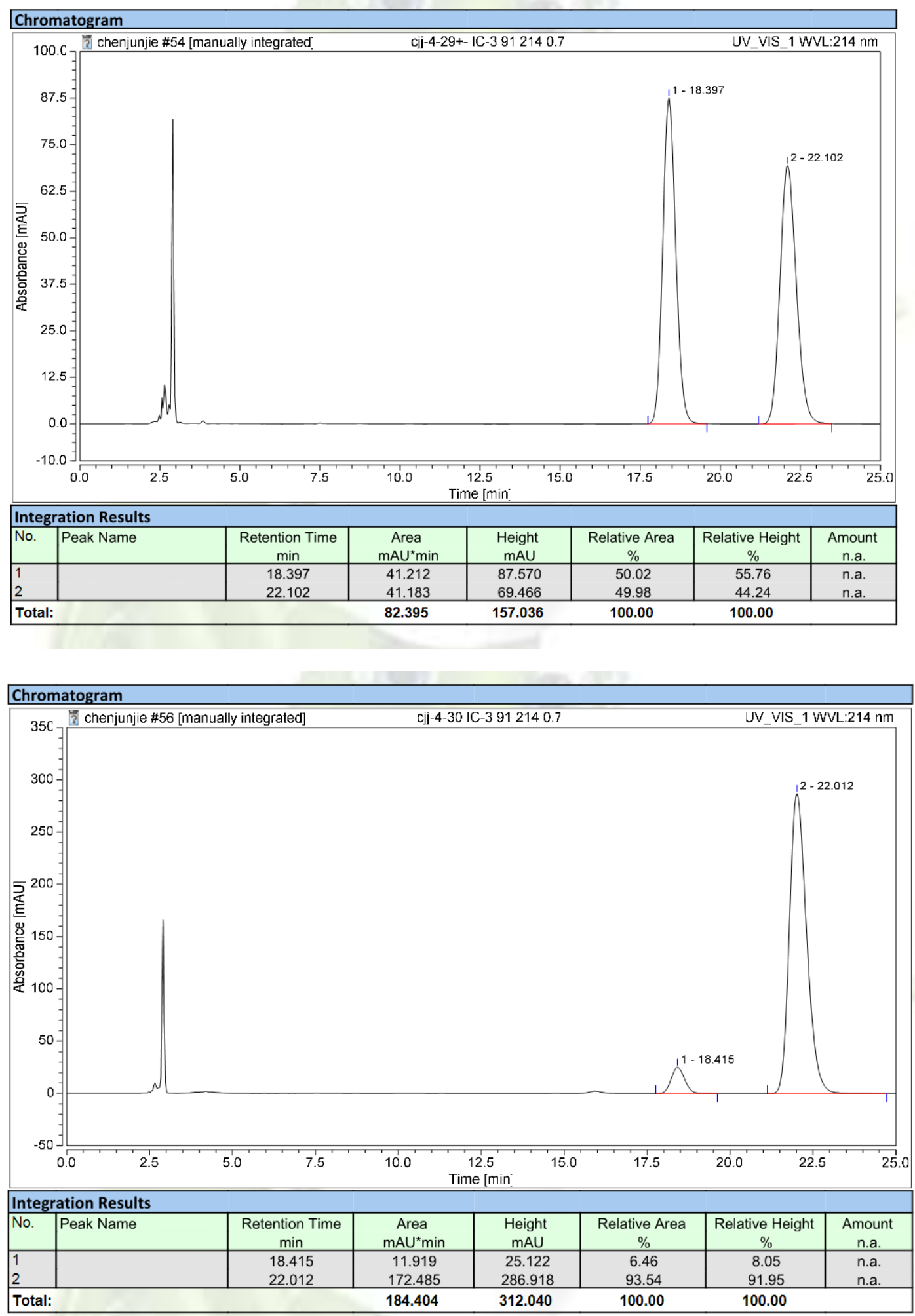
<smiles>CC(=O)C[C@@]1(C)/C(=C/[13CH3])CN([125I])c2ccccc21</smiles>

2n

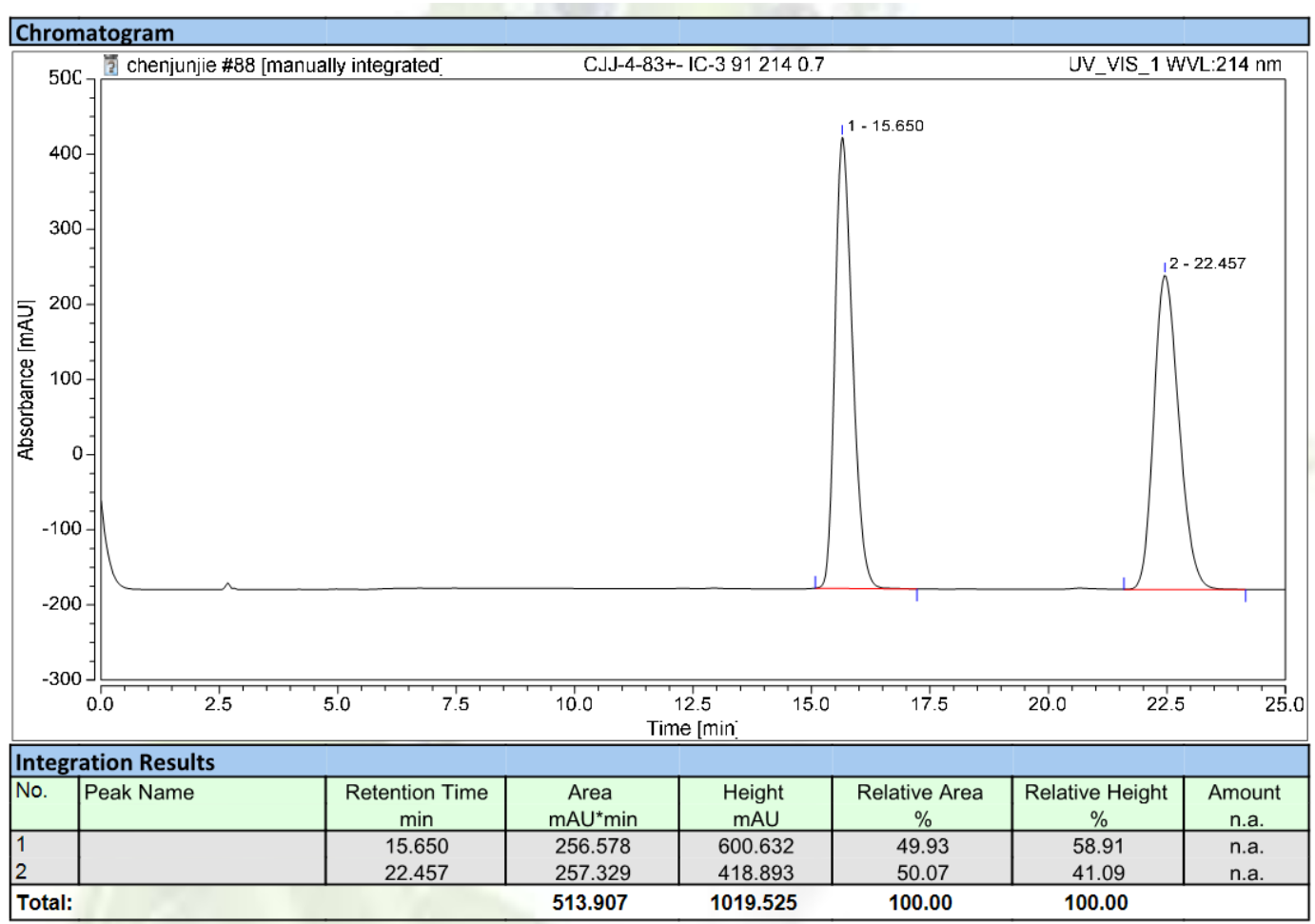

\section{Chromatogram}

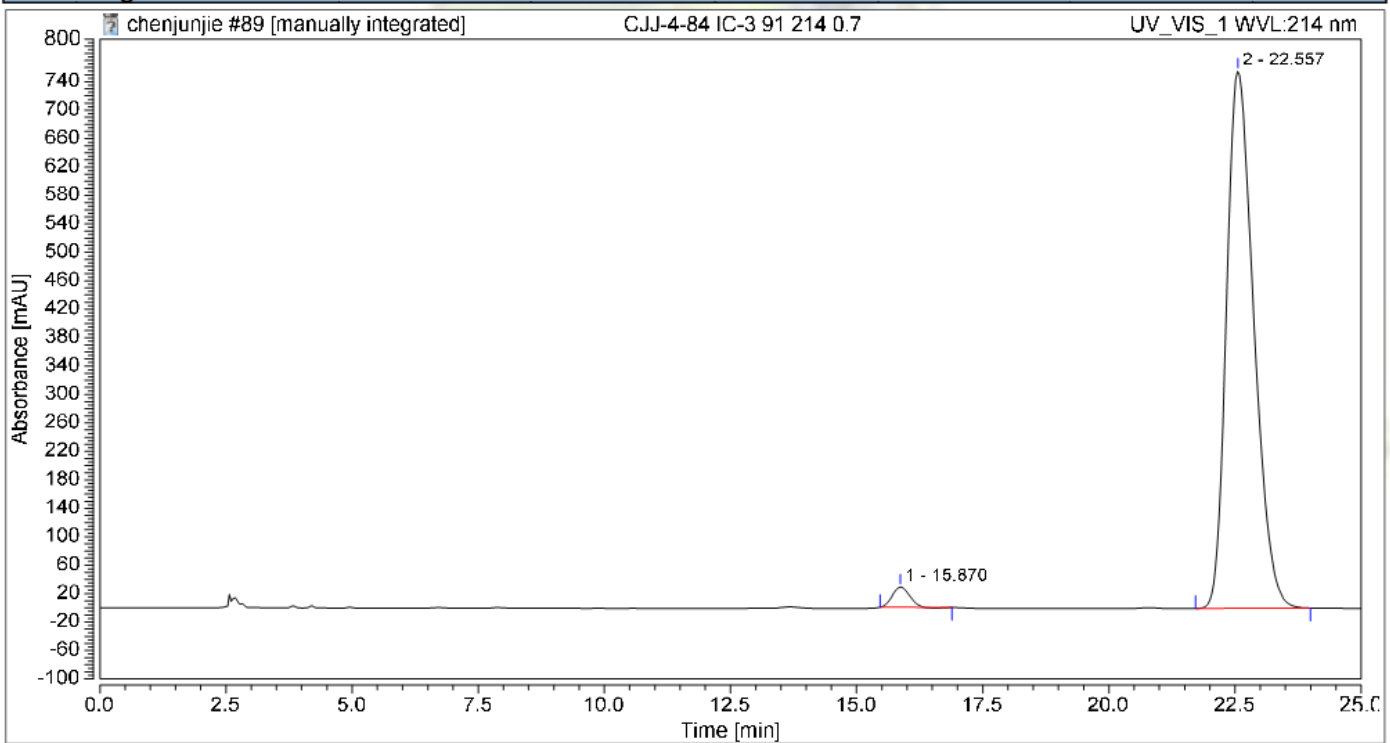

Integration Results

\begin{tabular}{|c|c|c|c|c|c|c|c|}
\hline No. & Peak Name & $\begin{array}{c}\text { Retention Time } \\
\text { min }\end{array}$ & $\begin{array}{c}\text { Area } \\
\mathrm{mAU}^{*} \min \end{array}$ & $\begin{array}{c}\text { Height } \\
\text { mAU }\end{array}$ & $\begin{array}{c}\text { Relative Area } \\
\% \\
\end{array}$ & \begin{tabular}{|c|} 
Relative Height \\
$\%$ \\
\end{tabular} & $\begin{array}{c}\text { Amount } \\
\text { n.a. }\end{array}$ \\
\hline $\begin{array}{l}1 \\
2\end{array}$ & & $\begin{array}{l}15.870 \\
22.557\end{array}$ & $\begin{array}{c}11.208 \\
472.974\end{array}$ & $\begin{array}{c}28.519 \\
755.576\end{array}$ & $\begin{array}{c}2.31 \\
97.69\end{array}$ & $\begin{array}{c}3.64 \\
96.36\end{array}$ & $\begin{array}{l}\text { n.a. } \\
\text { n.a. }\end{array}$ \\
\hline \multicolumn{3}{|c|}{ Total: } & 484.182 & 784.095 & 100.00 & 100.00 & \\
\hline
\end{tabular}


<smiles>CC(C)(C)/C=C1\CN([As])c2ccccc2[C@@]1(C)CC(=O)NC(C)(C)C</smiles>

20

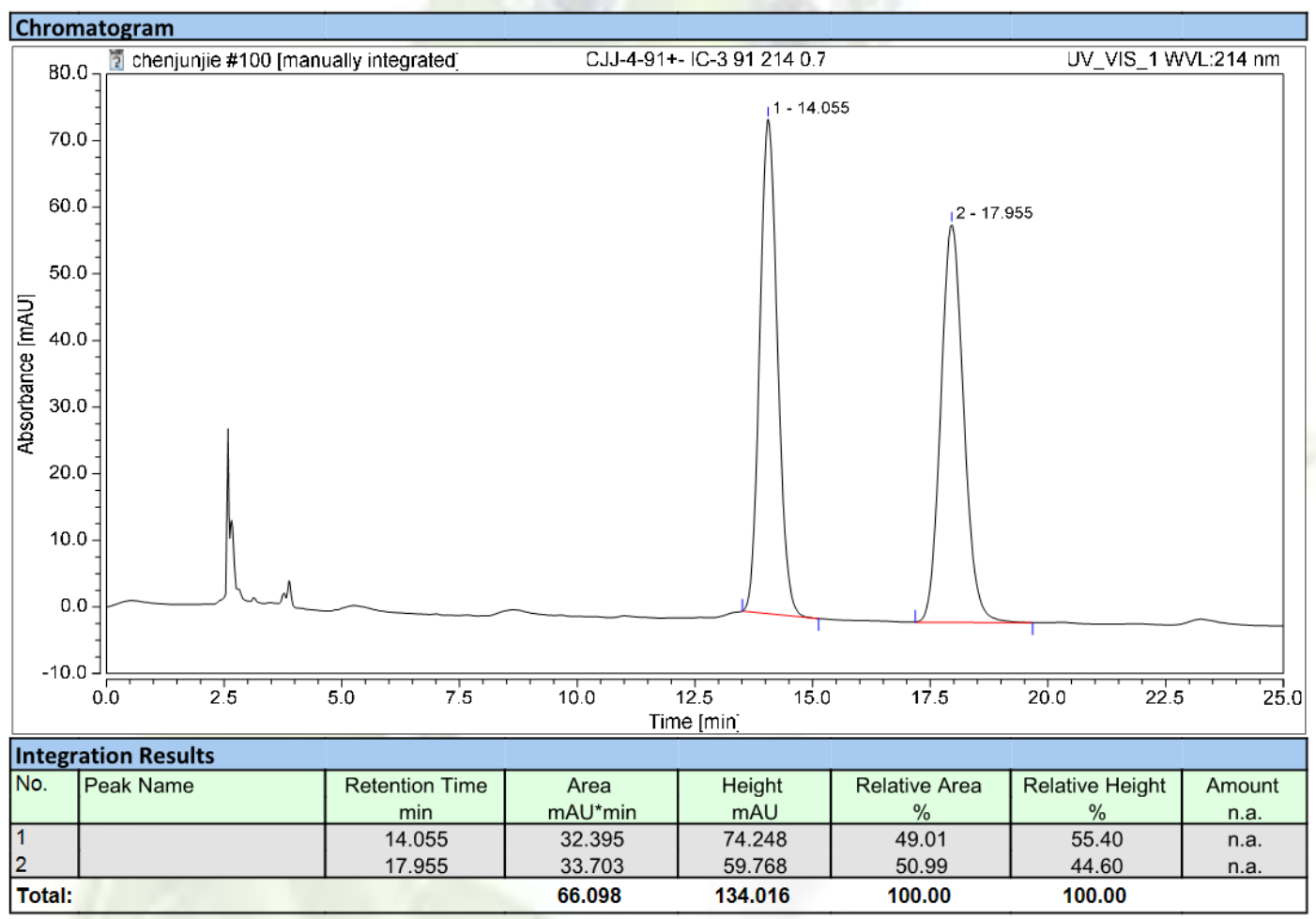

\section{Chromatogram}

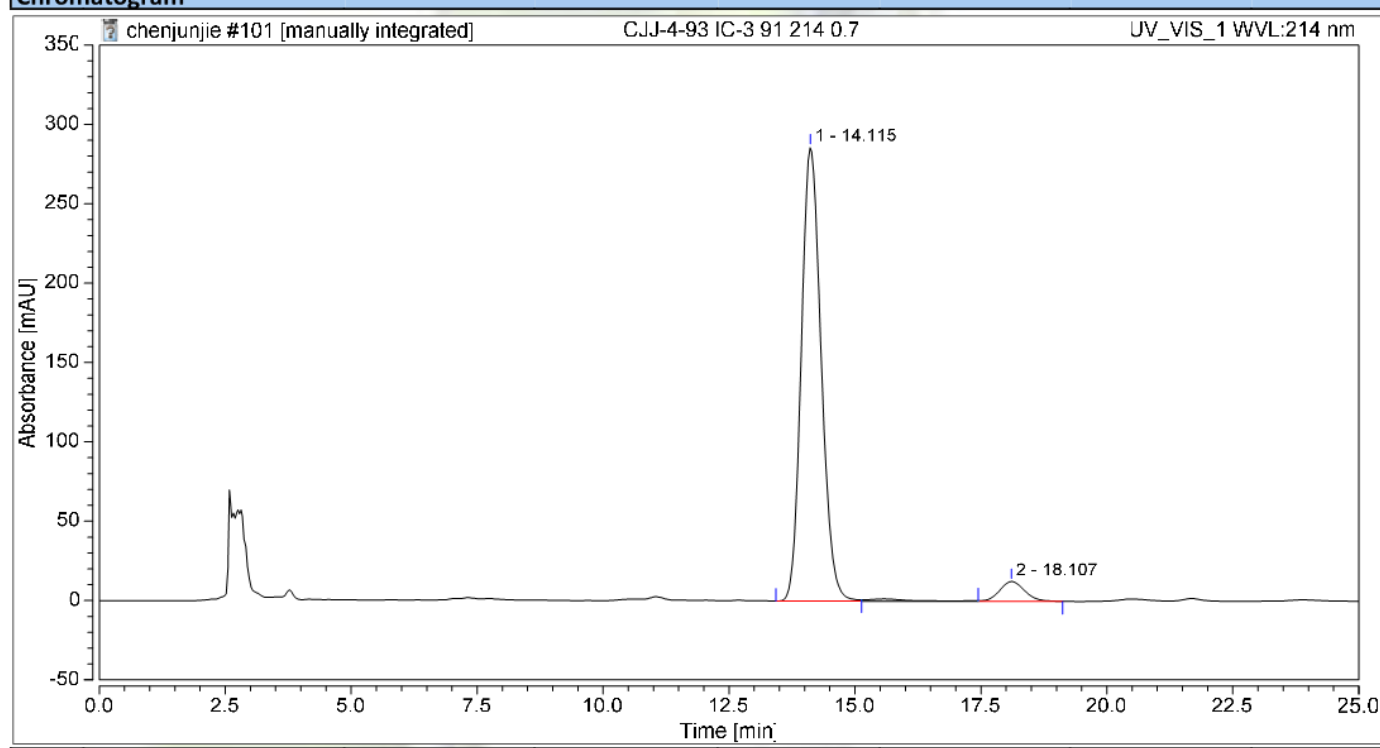

Integration Results

\begin{tabular}{|l|l|c|c|c|c|c|c|}
\hline No. & Peak Name & $\begin{array}{c}\text { Retention Time } \\
\min \end{array}$ & $\begin{array}{c}\text { Area } \\
\mathrm{mAU}^{*} \min \end{array}$ & $\begin{array}{c}\text { Height } \\
\mathrm{mAU}\end{array}$ & $\begin{array}{c}\text { Relative Area } \\
\%\end{array}$ & $\begin{array}{c}\text { Relative Height } \\
\%\end{array}$ & $\begin{array}{c}\text { Amount } \\
\text { n.a. }\end{array}$ \\
\hline 1 & & 14.115 & 130.448 & 286.112 & 94.75 & 95.81 & n.a. \\
2 & & 18.107 & 7.224 & 12.501 & 5.25 & 4.19 & n.a. \\
\hline
\end{tabular}


<smiles>CC[C@@]1(CC(C)=O)C(=CC(C)=O)C[NH2+]c2ccccc21</smiles>

$2 p$
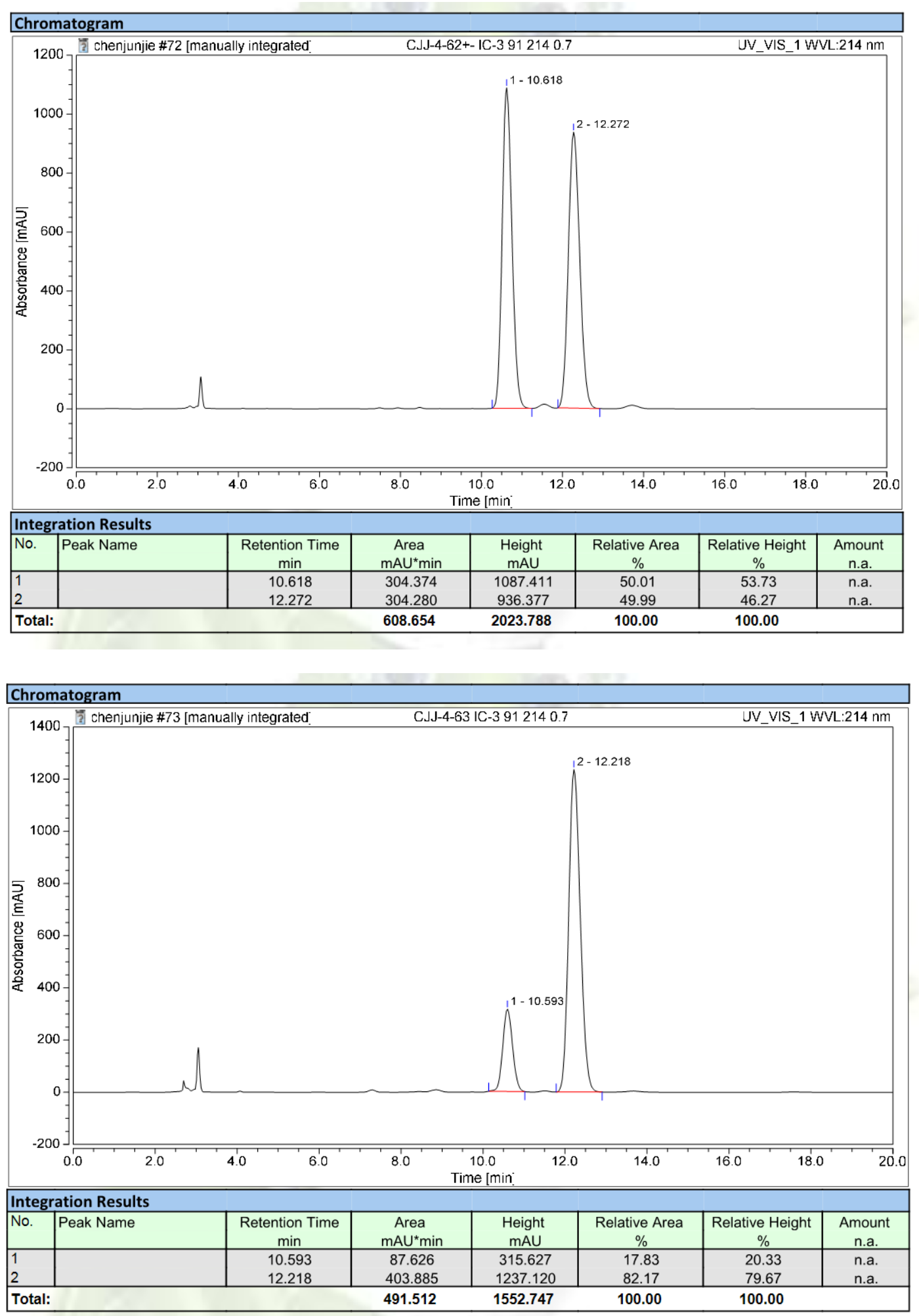
<smiles></smiles>

$4 a$

\section{Chromatogram}

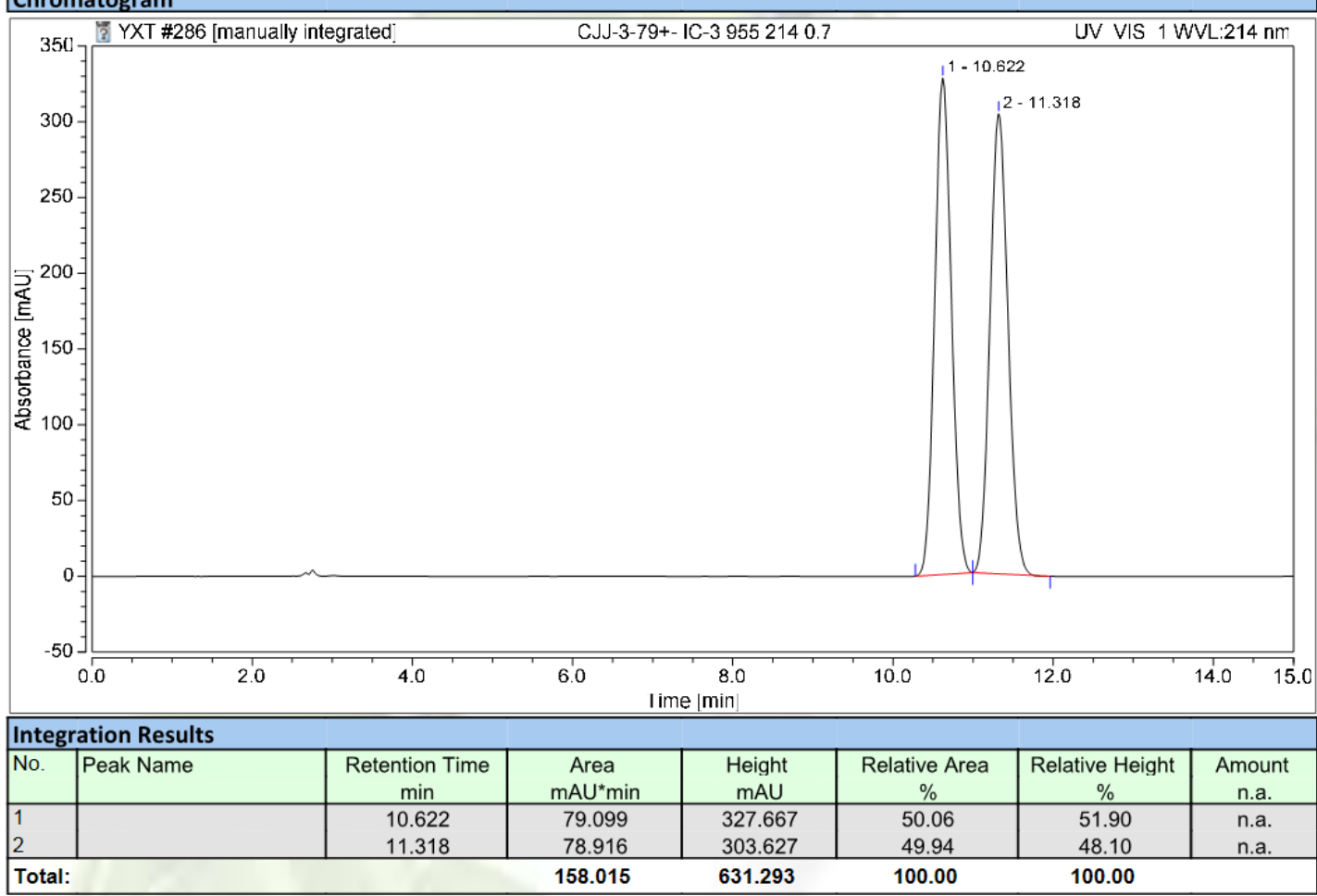

\section{Chromatogram}

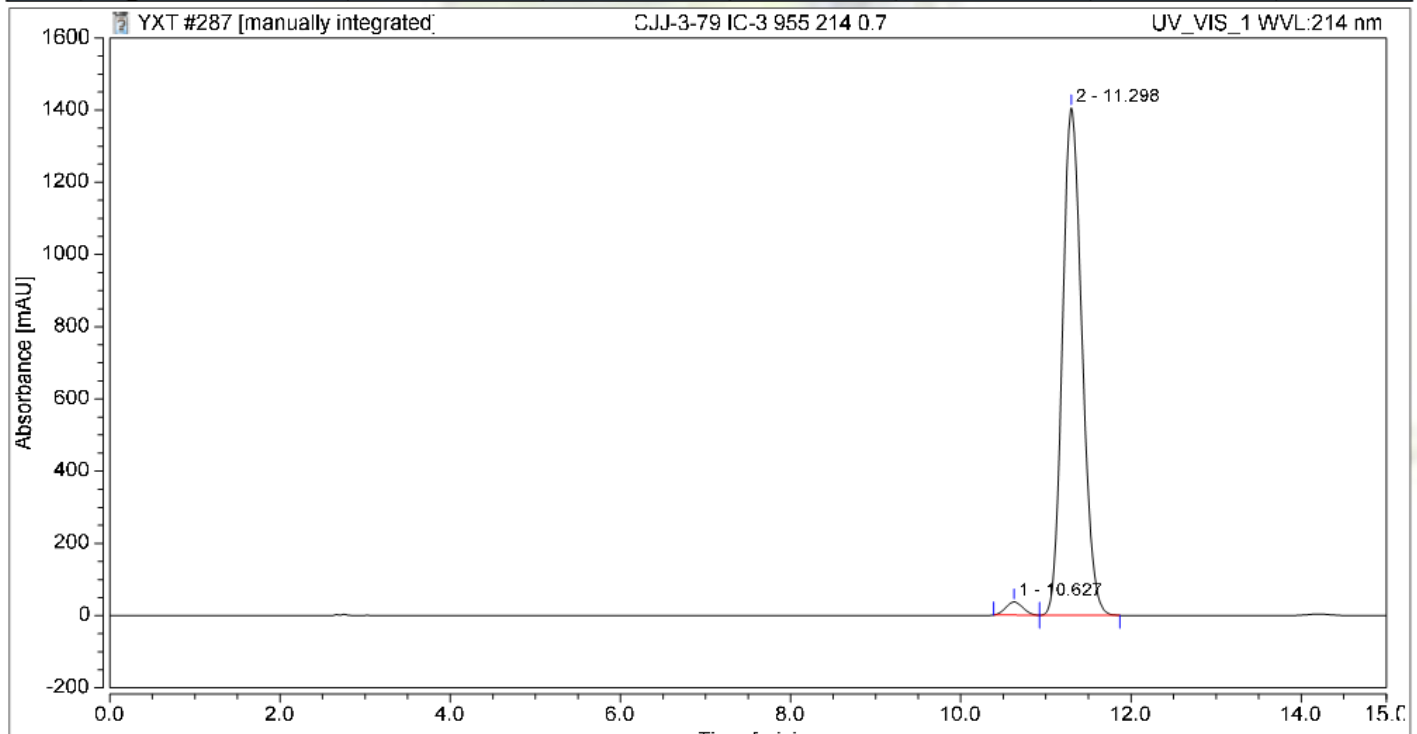

\begin{tabular}{|c|c|c|c|c|c|c|c|}
\hline \multicolumn{8}{|c|}{ Integration Results } \\
\hline No. & Peak Name & $\begin{array}{c}\text { Retention Time } \\
\text { min }\end{array}$ & $\begin{array}{c}\text { Area } \\
\mathrm{mAU}^{*} \min \end{array}$ & $\begin{array}{c}\text { Height } \\
\text { mAU }\end{array}$ & $\begin{array}{c}\text { Relative Area } \\
\%\end{array}$ & $\begin{array}{c}\text { Relative Height } \\
\%\end{array}$ & $\begin{array}{c}\text { Amount } \\
\text { n.a. }\end{array}$ \\
\hline $\begin{array}{l}1 \\
2\end{array}$ & & $\begin{array}{l}10.627 \\
11.298\end{array}$ & $\begin{array}{c}8.544 \\
374.101\end{array}$ & $\begin{array}{c}36.672 \\
1405.742\end{array}$ & $\begin{array}{c}2.23 \\
97.77\end{array}$ & $\begin{array}{c}2.54 \\
97.46\end{array}$ & $\begin{array}{l}\text { n.a. } \\
\text { n.a. }\end{array}$ \\
\hline \multicolumn{3}{|c|}{ Total: } & 382.645 & 1442.414 & 100.00 & 100.00 & \\
\hline
\end{tabular}


<smiles>C=C[C@]1(C)/C(=C/C(C)(C)C)CN([As])c2ccc(C)cc21</smiles>

4b

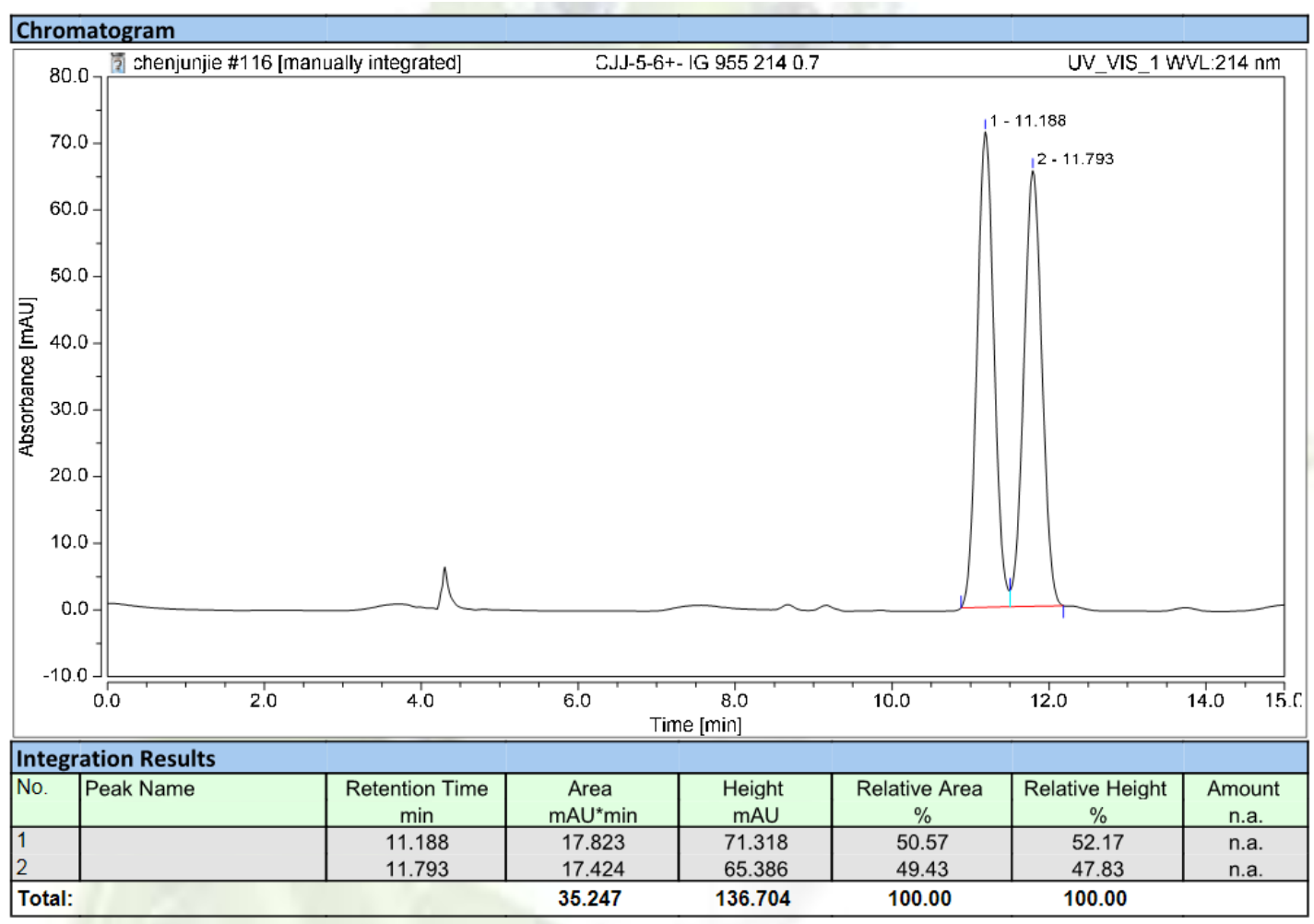

\section{Chromatogram}

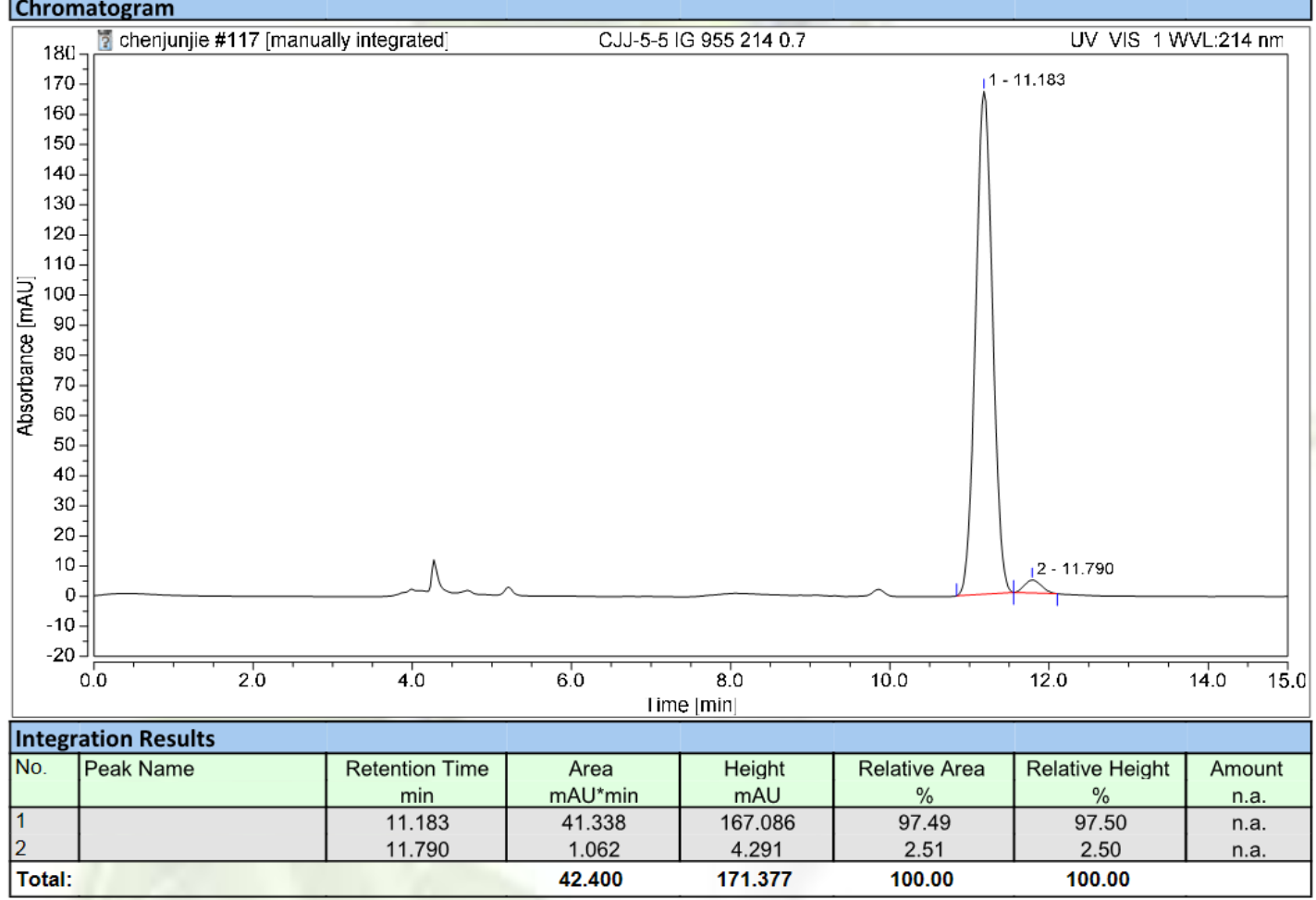


<smiles>C=C[C@]1(C)/C(=C/[Y5](C)(C)C)CN([As])c2cc(C)ccc21</smiles>

4c

\section{Chromatogram}

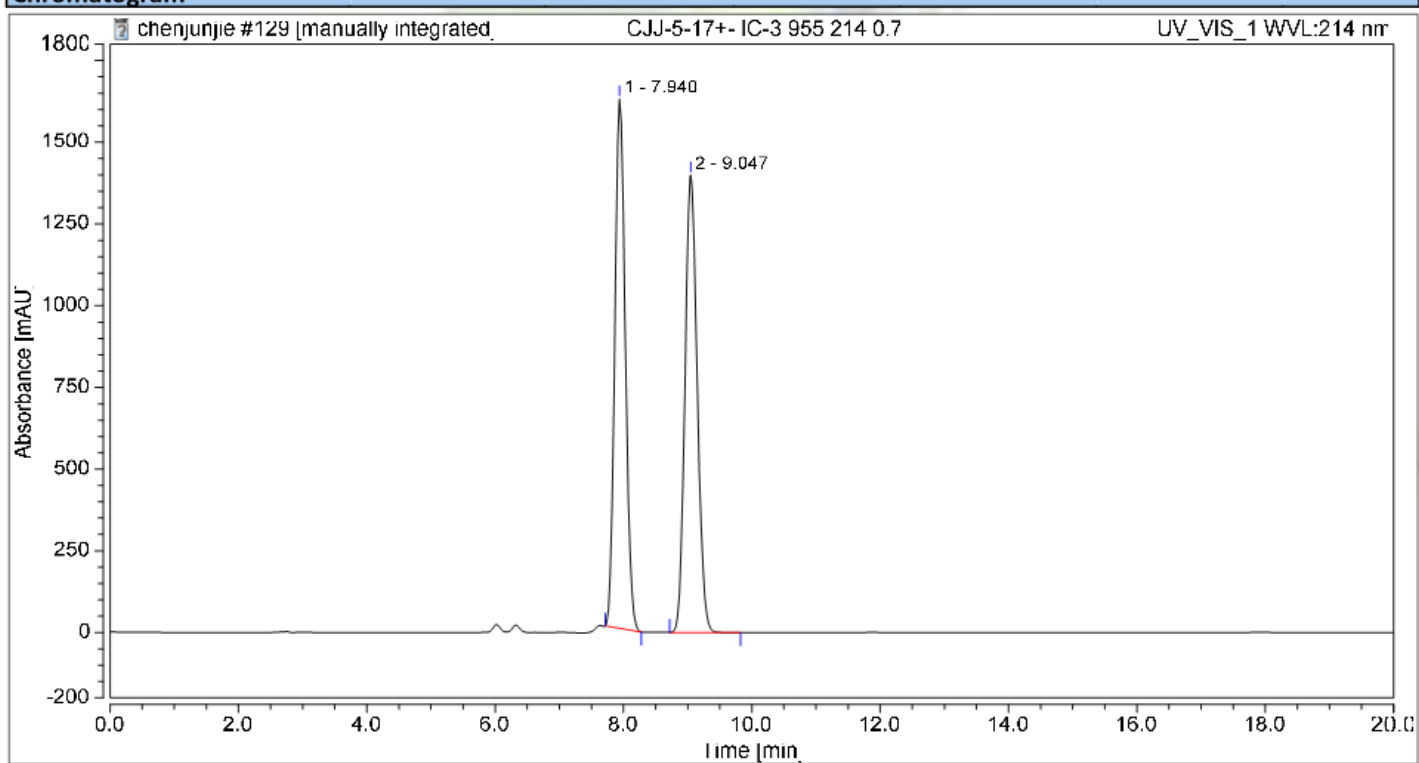

\begin{tabular}{|c|c|c|c|c|c|c|c|}
\hline \multicolumn{8}{|c|}{ Integration Results } \\
\hline No. & Peak Name & $\begin{array}{c}\text { Retention Time } \\
\text { min }\end{array}$ & $\begin{array}{c}\text { Area } \\
m A U^{\star} \text { min }\end{array}$ & $\begin{array}{l}\text { Height } \\
\text { mAU }\end{array}$ & $\begin{array}{c}\text { Relative Area } \\
\%\end{array}$ & $\begin{array}{c}\text { Relative Height } \\
\%\end{array}$ & $\begin{array}{c}\text { Amount } \\
\text { n.a. }\end{array}$ \\
\hline 1 & & 7.940 & 305.173 & 1619.449 & 49.81 & 53.65 & n.a. \\
\hline 2 & & 9.047 & 307.548 & 1399.186 & 50.19 & 46.35 & n.a. \\
\hline \multicolumn{3}{|c|}{ Total: } & 612.721 & 3018.636 & 100.00 & 100.00 & \\
\hline
\end{tabular}

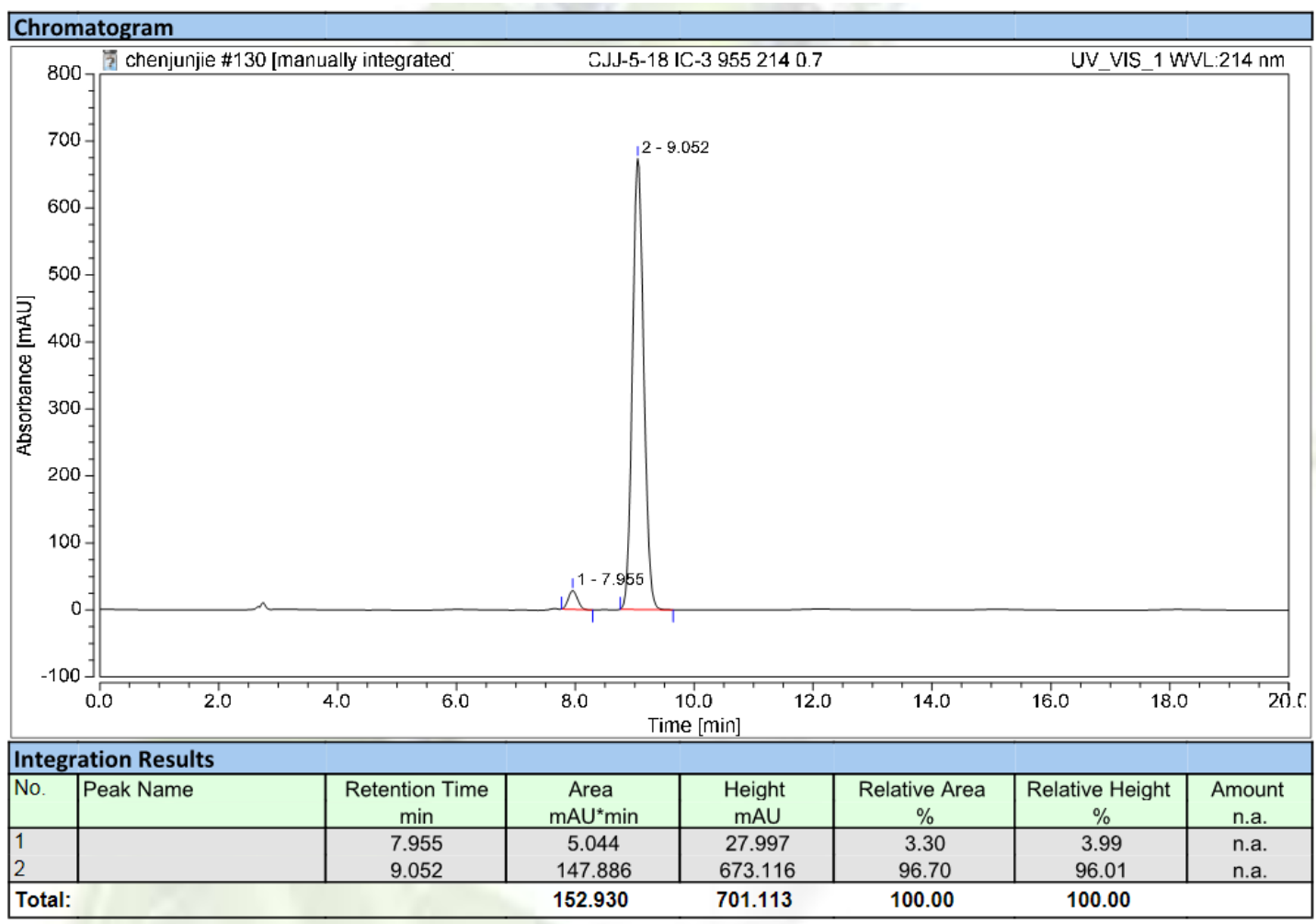


<smiles>C=C[C@]1(C)/C(=C/[Y15](C)(C)C)CN([As])c2ccc(OC)cc21</smiles>

4d
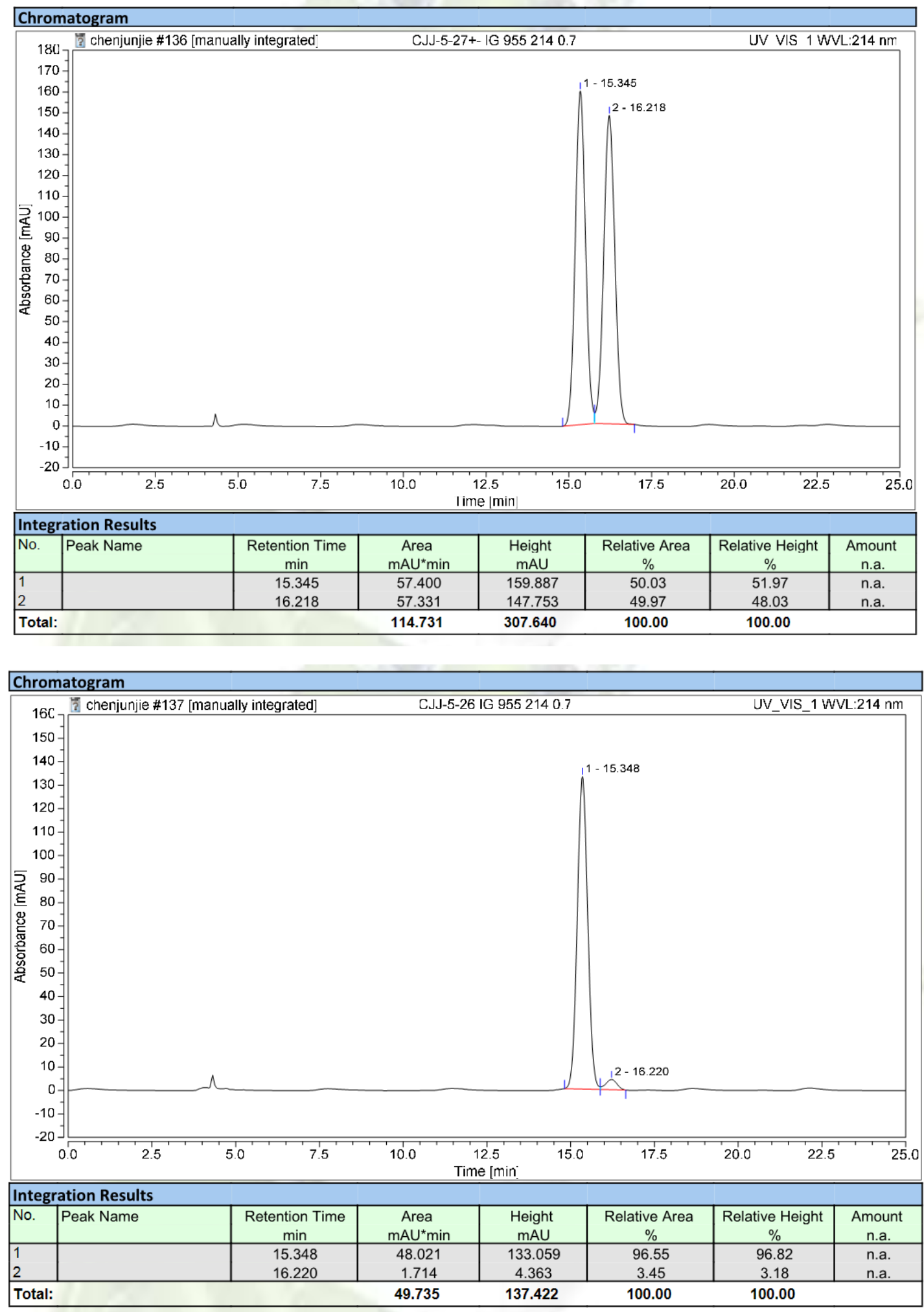
<smiles></smiles>

$4 e$

\section{Chromatogram}

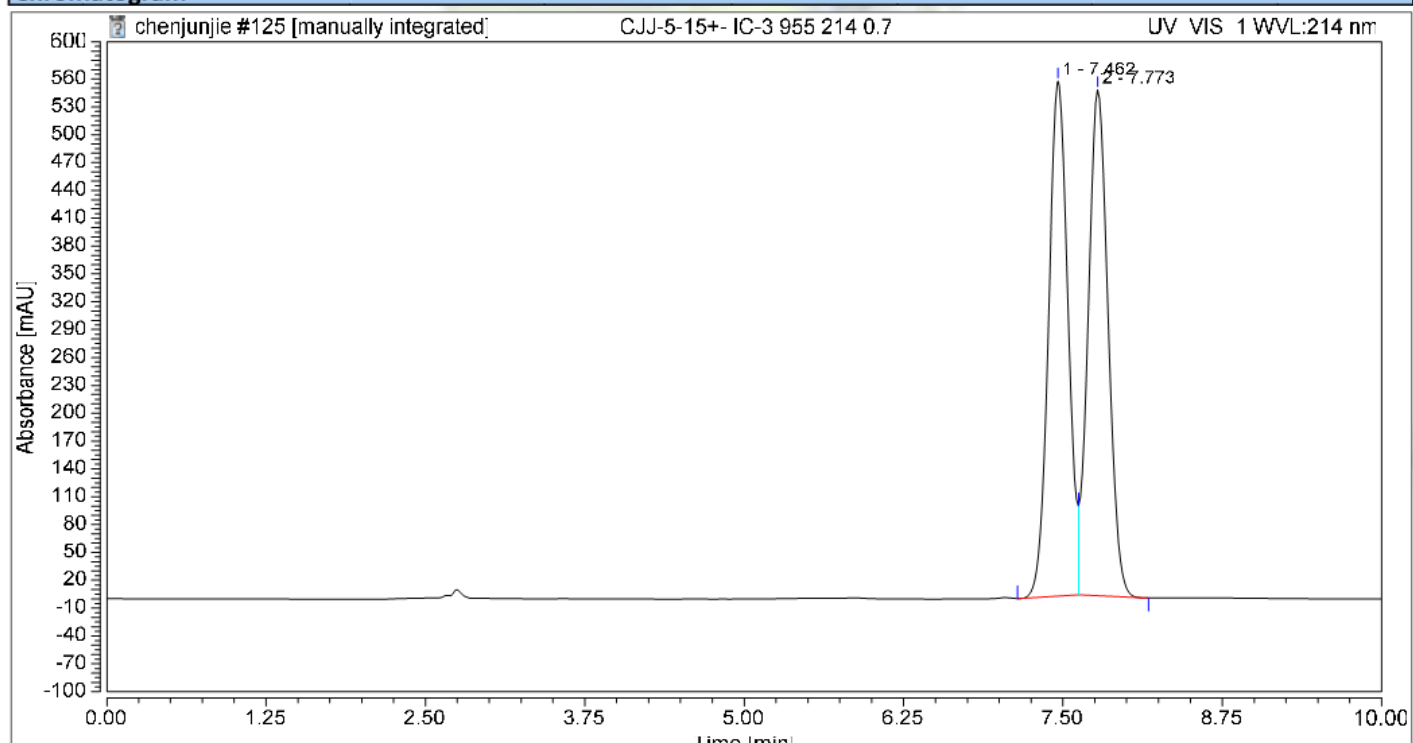

Integration Results

\begin{tabular}{|c|c|c|c|c|c|c|c|}
\hline No. & Peak Name & $\begin{array}{c}\text { Retention Time } \\
\text { min }\end{array}$ & $\begin{array}{c}\text { Area } \\
\mathrm{mAU}{ }^{*} \min \end{array}$ & $\begin{array}{c}\text { Height } \\
\text { mAU }\end{array}$ & $\begin{array}{c}\text { Relative Area } \\
\% \\
\end{array}$ & $\begin{array}{c}\text { Relative Height } \\
\%\end{array}$ & $\begin{array}{c}\text { Amount } \\
\text { n.a. }\end{array}$ \\
\hline 1 & & 7.462 & 97.289 & 554.687 & 49.69 & 50.44 & n.a. \\
\hline 2 & & 7.773 & 98.511 & 545.031 & 50.31 & 49.56 & n.a. \\
\hline \multicolumn{3}{|c|}{ Total: } & 195.800 & 1099.718 & 100.00 & 100.00 & \\
\hline
\end{tabular}

\section{Chromatogram}

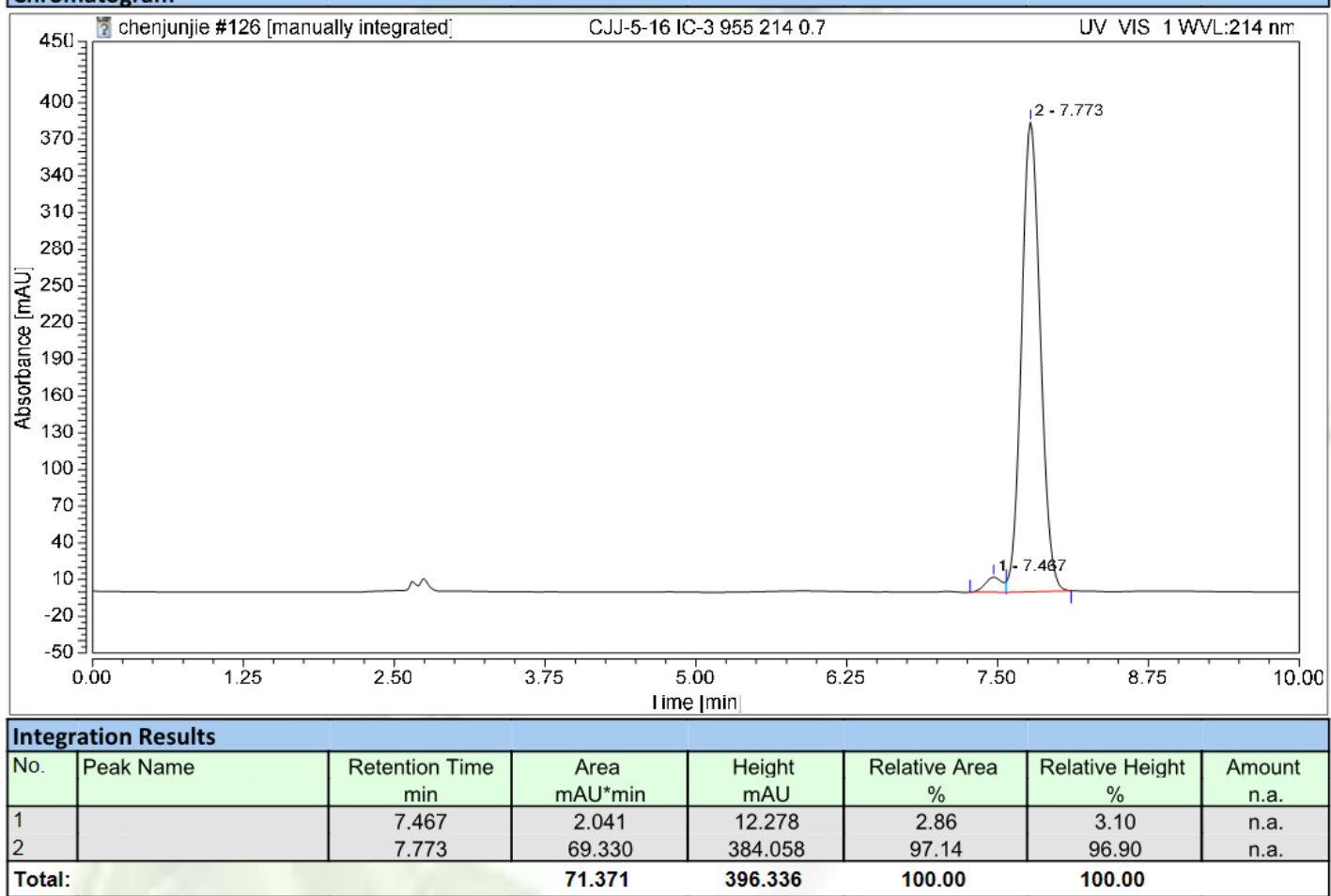


<smiles>C=C[C@]1(C)/C(=C/[Y15](=O)[O-])CN([As])c2ccc(Cl)cc21</smiles>

$4 f$

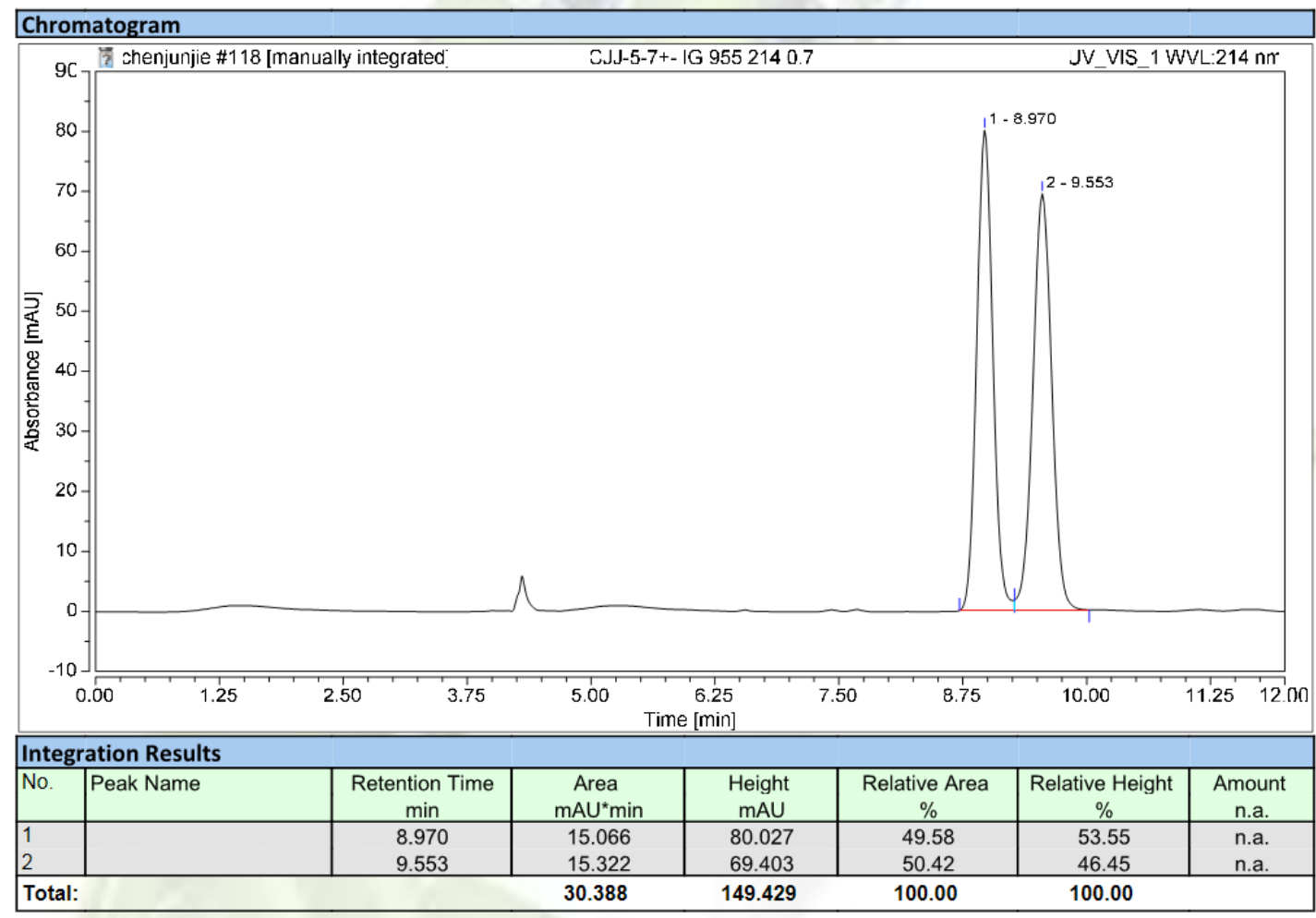

Chromatogram

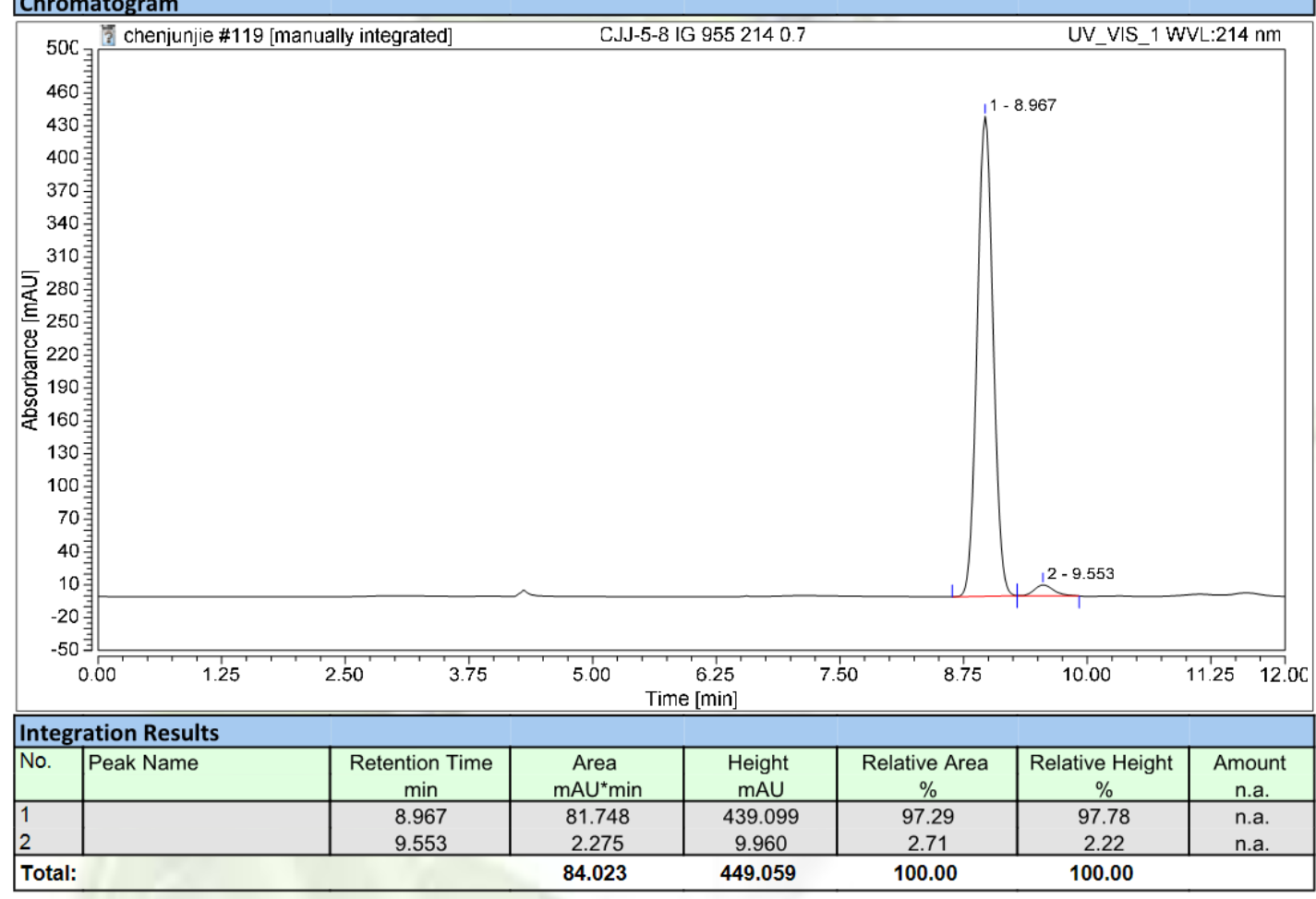


<smiles>C=C[C@]1(C)/C(=C/[Y15](C)(C)C)CN([As])c2ccc(Br)cc21</smiles>

$4 g$

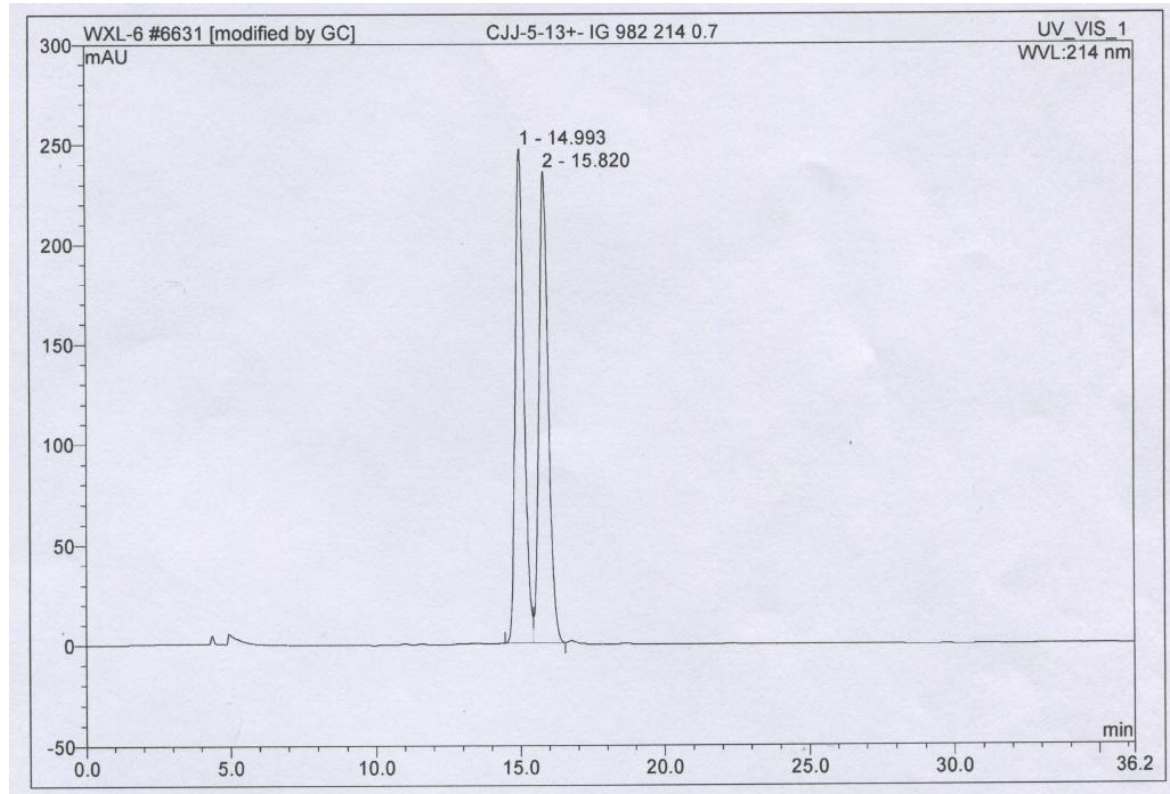

\begin{tabular}{|r|cccrrrr|}
\hline No. & $\begin{array}{c}\text { Ret.Time } \\
\text { min }\end{array}$ & Peak Name & Height & Area & Rel.Area & Amount & Type \\
& 14.99 & n.a. & 246.887 & 87.154 & 49.60 & n.a. & BM \\
\hline 1 & 15.82 & n.a. & 235.463 & 88.548 & 50.40 & n.a. & MB \\
\hline Total: & & & 482.350 & 175.702 & 100.00 & 0.000 & \\
\hline
\end{tabular}

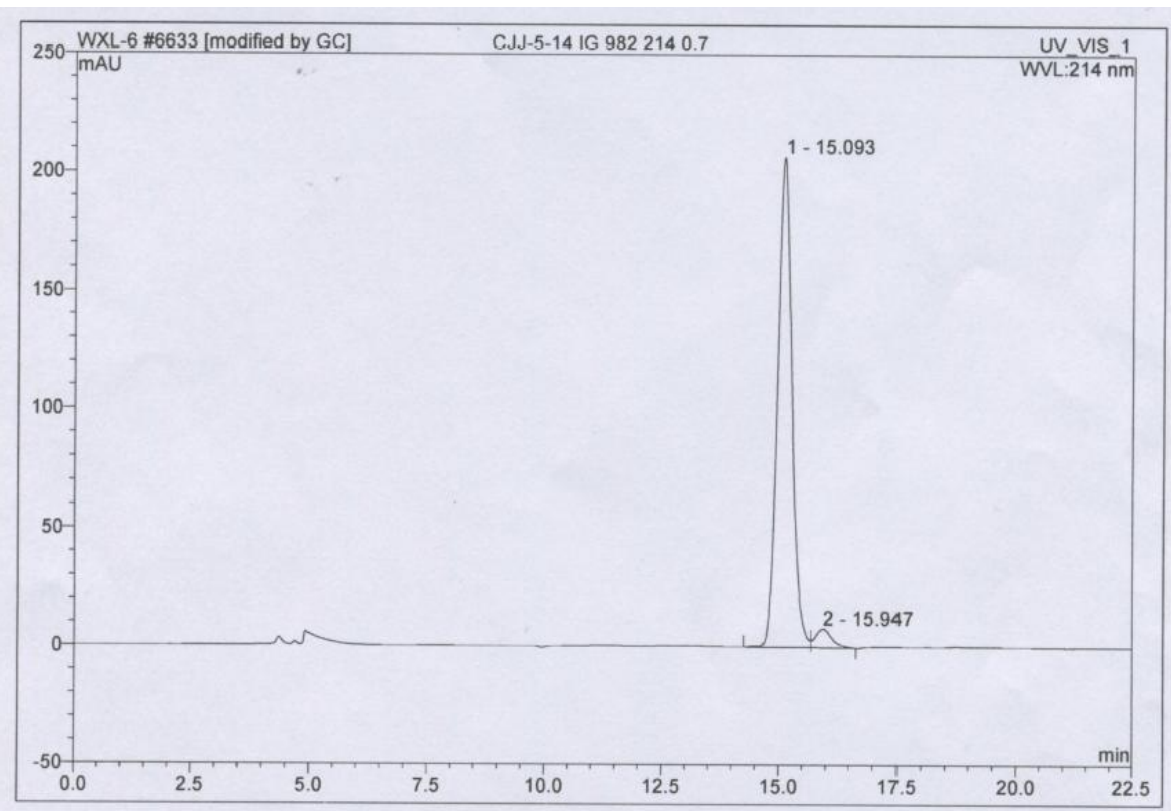

\begin{tabular}{|r|rrrrrrr|}
\hline No. & $\begin{array}{c}\text { Ret.Time } \\
\text { min }\end{array}$ & Peak Name & $\begin{array}{c}\text { Height } \\
\text { mAU }\end{array}$ & $\begin{array}{c}\text { Area } \\
\text { mAU*min }\end{array}$ & $\begin{array}{c}\text { Rel.Area } \\
\%\end{array}$ & Amount & Type \\
\hline 1 & 15.09 & n.a. & 206.591 & 73.533 & 95.97 & n.a. & BM \\
2 & 15.95 & n.a. & 7.733 & 3.086 & 4.03 & n.a. & MB \\
\hline Total: & & & 214.324 & 76.619 & 100.00 & 0.000 & \\
\hline
\end{tabular}


<smiles>C=C[C@]1(C)/C(=C/[15C](C)(C)C)CN([As])c2ccc(C(F)(F)F)cc21</smiles>

4h

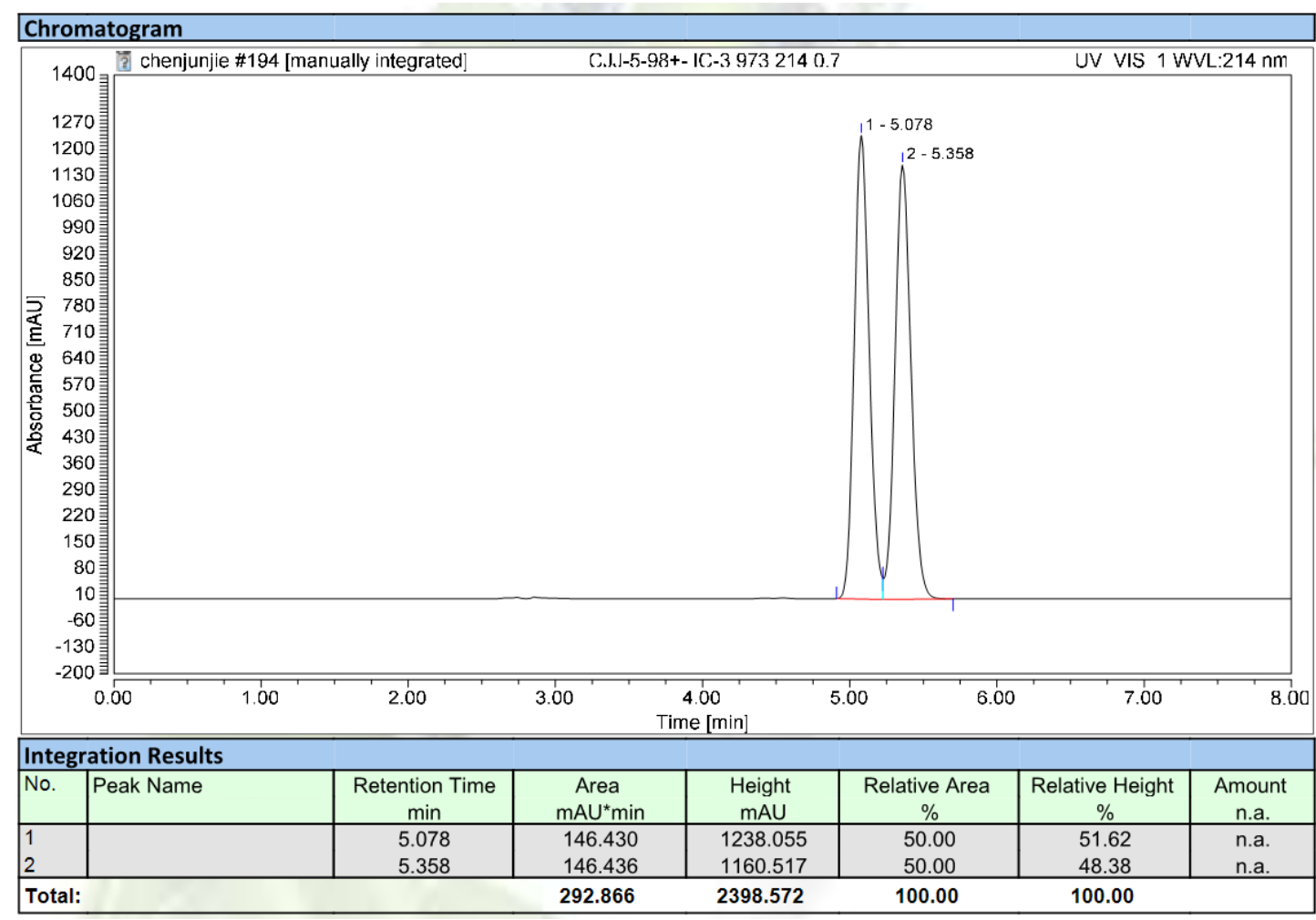

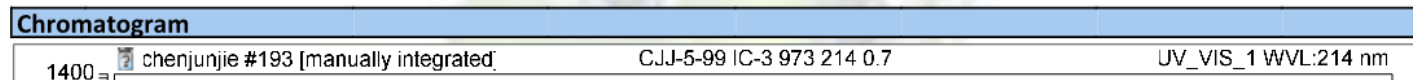

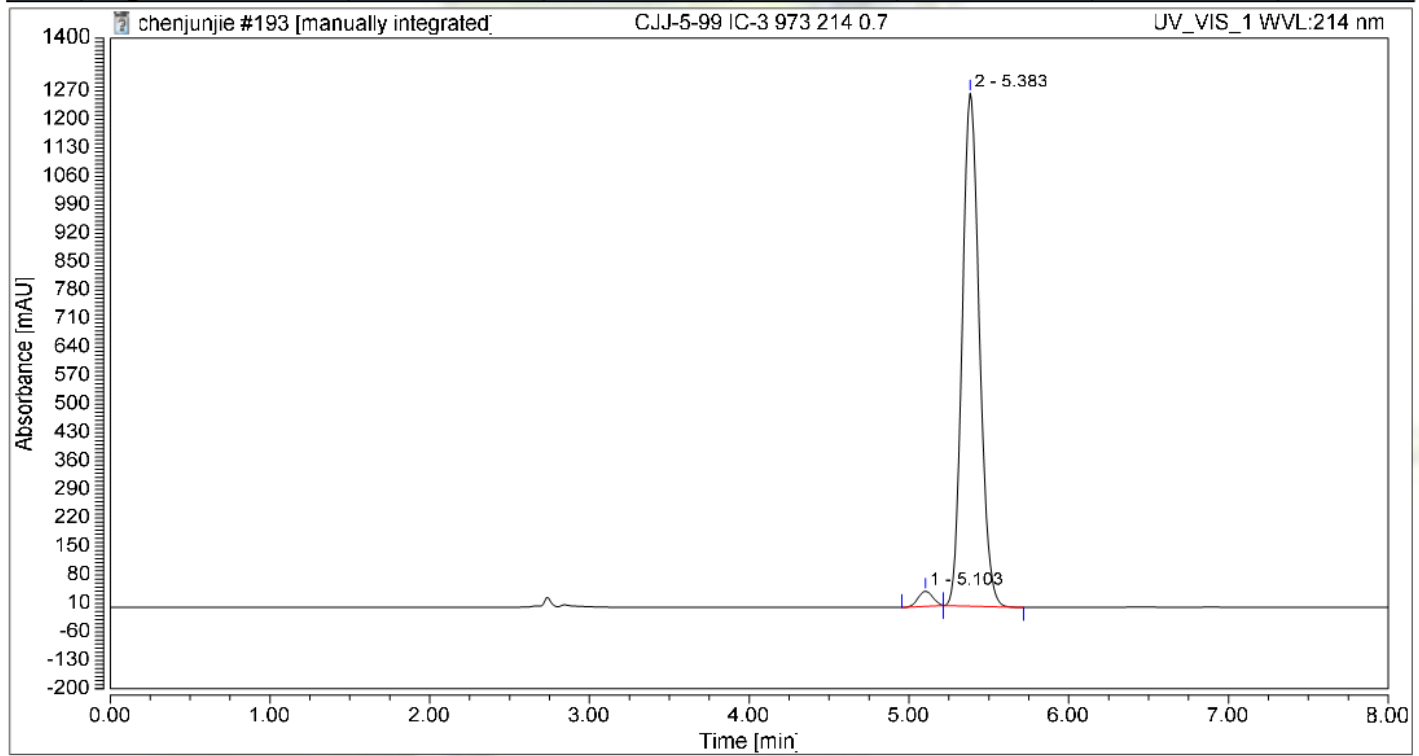

\begin{tabular}{|l|l|c|c|c|c|c|c|}
\hline Integration Results \\
\hline No. & Peak Name & $\begin{array}{c}\text { Retention Time } \\
\text { min }\end{array}$ & $\begin{array}{c}\text { Area } \\
\mathrm{mAU} \text { min }\end{array}$ & $\begin{array}{c}\text { Height } \\
\mathrm{mAU}\end{array}$ & $\begin{array}{c}\text { Relative Area } \\
\%\end{array}$ & $\begin{array}{c}\text { Relative Height } \\
\%\end{array}$ & $\begin{array}{c}\text { Amount } \\
\text { n.a. }\end{array}$ \\
\hline 1 & & 5.103 & 3.972 & 37.141 & 2.47 & 2.86 & n.a. \\
2 & 5.383 & 156.775 & 1261.275 & 97.53 & 97.14 & n.a. \\
\hline Total: & 160.747 & 1298.416 & 100.00 & 100.00 & \\
\hline
\end{tabular}


<smiles>C=C[C@]1(C)/C(=C/[13C](C)C)CN([As])c2ccccc21</smiles>

$4 i$

\section{Chromatogram}

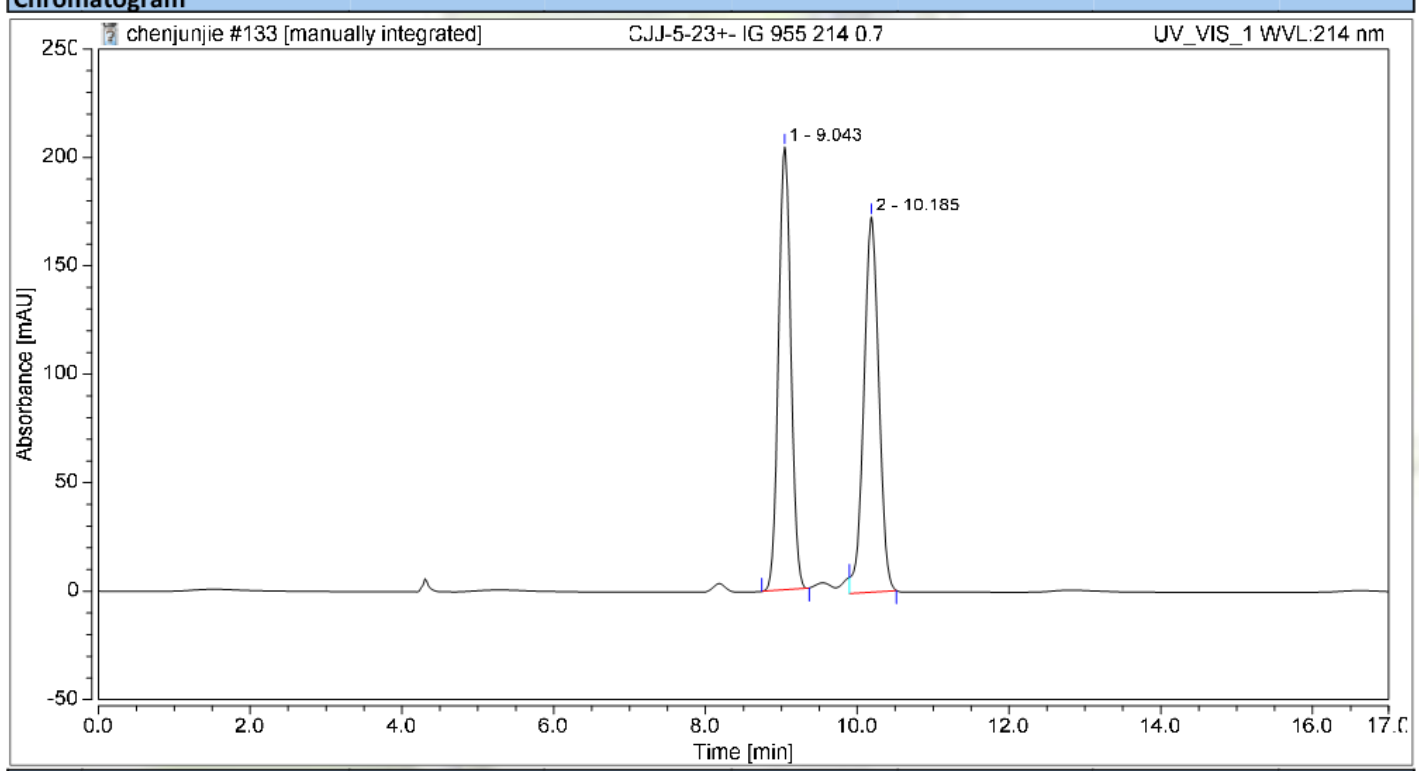

\begin{tabular}{|l|c|c|c|c|c|c|c|}
\hline Integration Results \\
\hline No. & Peak Name & $\begin{array}{c}\text { Retention Time } \\
\text { min }\end{array}$ & $\begin{array}{c}\text { Area } \\
\mathrm{mAU} \text { min }\end{array}$ & $\begin{array}{c}\text { Height } \\
\mathrm{mAU}\end{array}$ & $\begin{array}{c}\text { Relative Area } \\
\%\end{array}$ & $\begin{array}{c}\text { Relative Height } \\
\%\end{array}$ & $\begin{array}{c}\text { Amount } \\
\text { n.a. }\end{array}$ \\
\hline 1 & & 9.043 & 38.503 & 204.164 & 49.31 & 54.13 & n.a. \\
2 & 10.185 & 39.573 & 173.014 & 50.69 & 45.87 & n.a. \\
\hline Total: & 78.076 & 377.178 & 100.00 & 100.00 \\
\hline
\end{tabular}

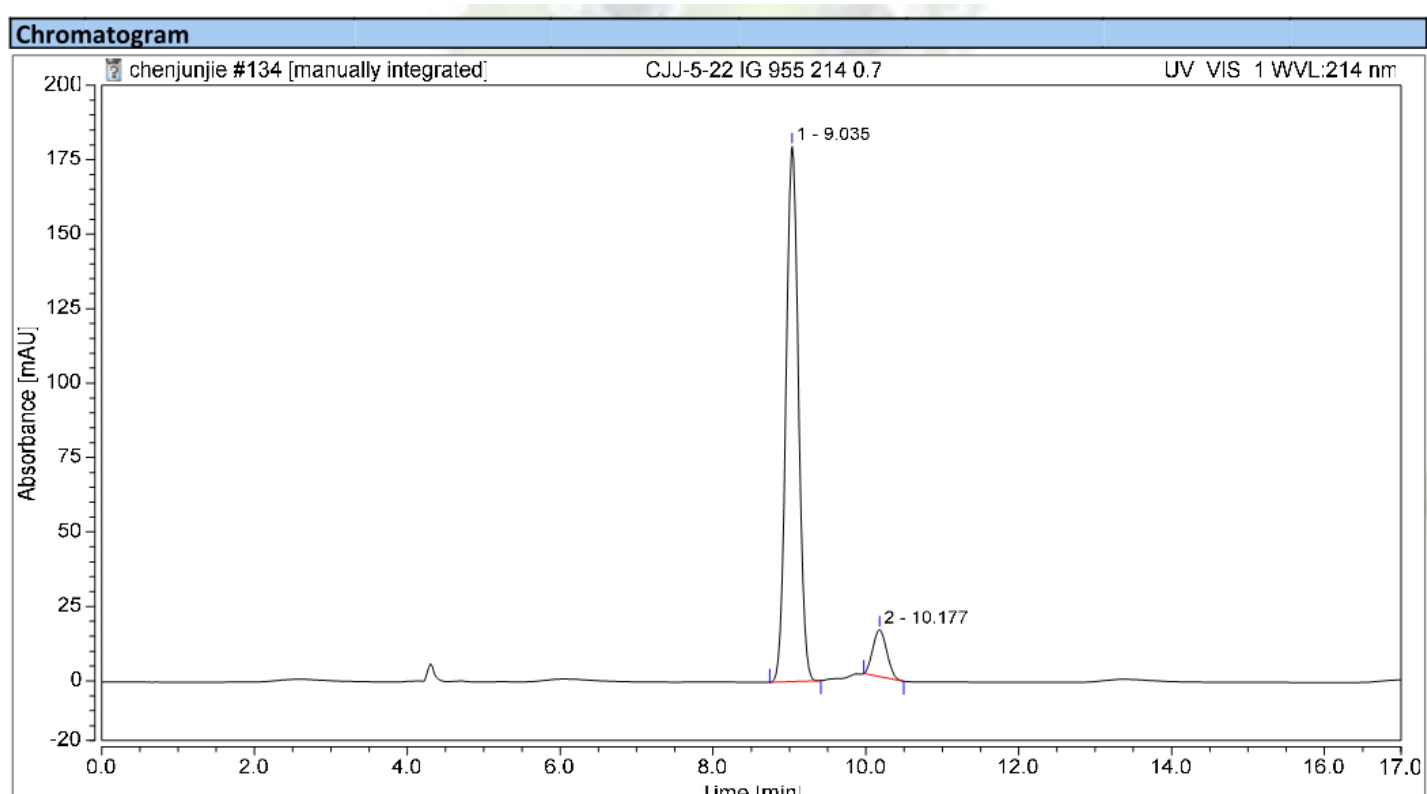

\begin{tabular}{|c|c|c|c|c|c|c|c|}
\hline \multicolumn{8}{|c|}{ Integration Results } \\
\hline No. & Peak Name & $\begin{array}{c}\text { Retention Time } \\
\text { min }\end{array}$ & $\begin{array}{c}\text { Area } \\
\mathrm{mAU}^{*} \min \end{array}$ & $\begin{array}{c}\text { Height } \\
\text { mAU }\end{array}$ & $\begin{array}{c}\text { Relative Area } \\
\%\end{array}$ & $\begin{array}{c}\text { Relative Height } \\
\%\end{array}$ & $\begin{array}{c}\text { Amount } \\
\text { n.a. }\end{array}$ \\
\hline $\begin{array}{l}1 \\
2\end{array}$ & & $\begin{array}{c}9.035 \\
10.177\end{array}$ & $\begin{array}{c}33.865 \\
3.321\end{array}$ & $\begin{array}{c}179.711 \\
15.801\end{array}$ & $\begin{array}{l}91.07 \\
8.93\end{array}$ & $\begin{array}{c}91.92 \\
8.08\end{array}$ & $\begin{array}{l}\text { n.a. } \\
\text { n.a. }\end{array}$ \\
\hline \multicolumn{3}{|c|}{ Total: } & 37.186 & 195.511 & 100.00 & 100.00 & \\
\hline
\end{tabular}


<smiles>C=C[C@]1(C)/C(=C/C(C)C)CN([As])c2ccccc21</smiles>

4j

\section{Chromatogram}

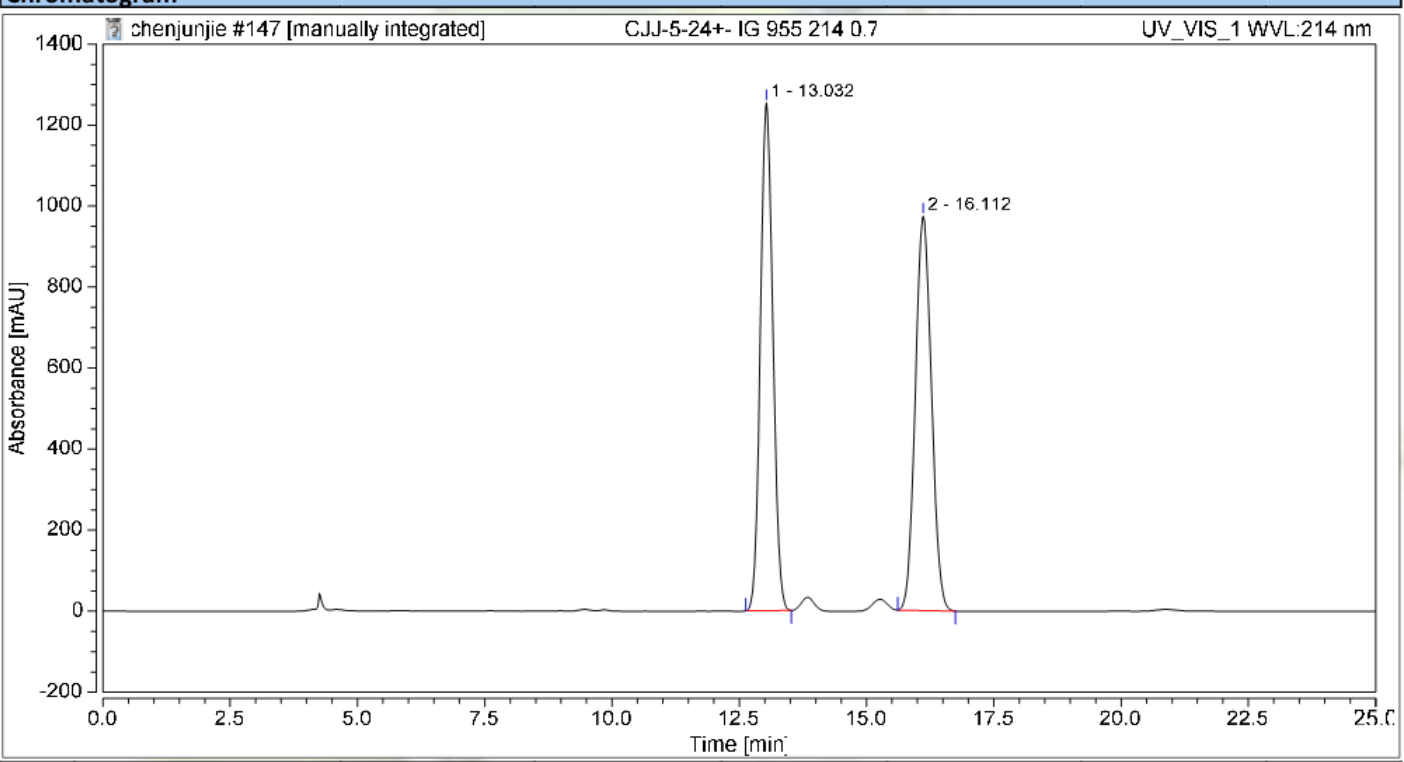

\begin{tabular}{|l|l|c|c|c|c|c|c|}
\hline Integration Results \\
\hline No. & Peak Name & $\begin{array}{c}\text { Retention Time } \\
\mathrm{min}\end{array}$ & $\begin{array}{c}\text { Area } \\
\mathrm{mAU} \text { min }\end{array}$ & $\begin{array}{c}\text { Height } \\
\mathrm{mAU}\end{array}$ & $\begin{array}{c}\text { Relative Area } \\
\%\end{array}$ & $\begin{array}{c}\text { Relative Height } \\
\%\end{array}$ & $\begin{array}{c}\text { Amount } \\
\text { n.a. }\end{array}$ \\
\hline 1 & 13.032 & 356.396 & 1253.882 & 49.91 & 56.29 & n.a. \\
2 & 16.112 & 357.736 & 973.853 & 50.09 & 43.71 & n.a. \\
\hline \multicolumn{7}{|l|}{} \\
\hline
\end{tabular}

\section{Chromatogram}

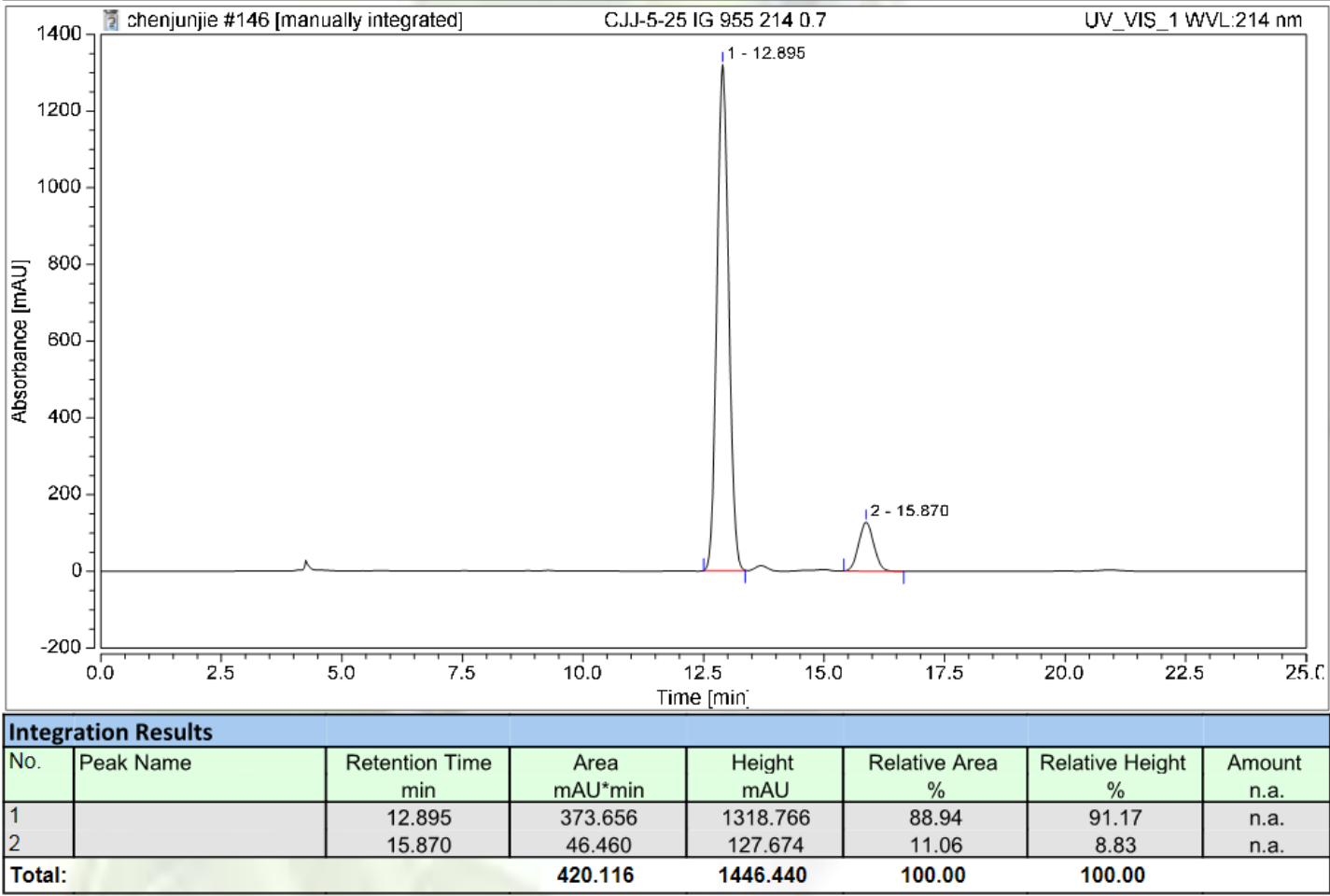




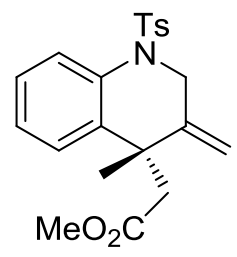

$5 a$
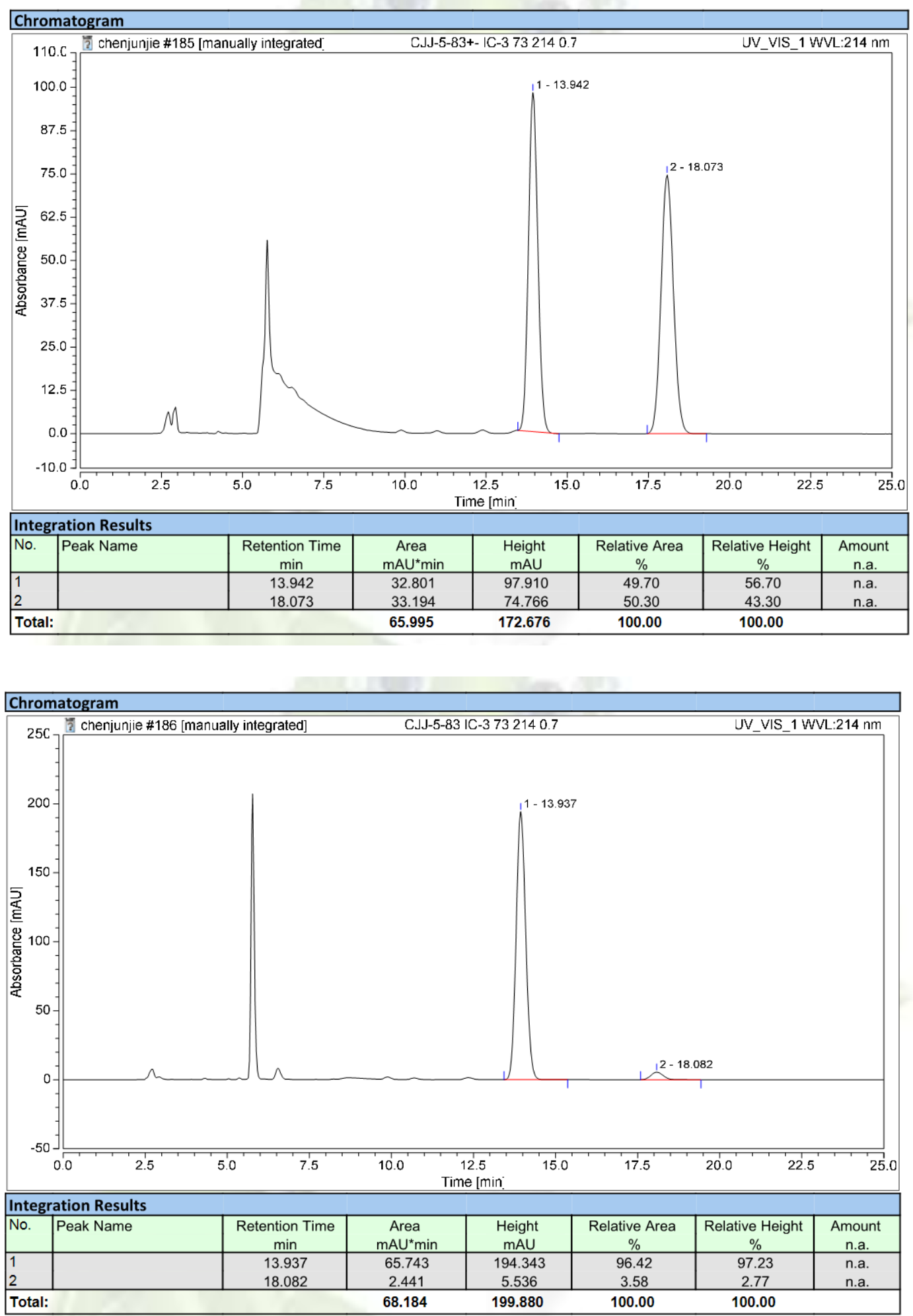


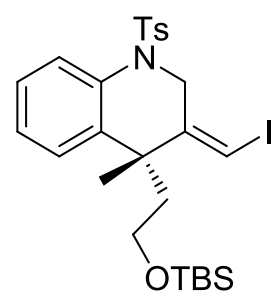

$6 a$

\section{Chromatogram}

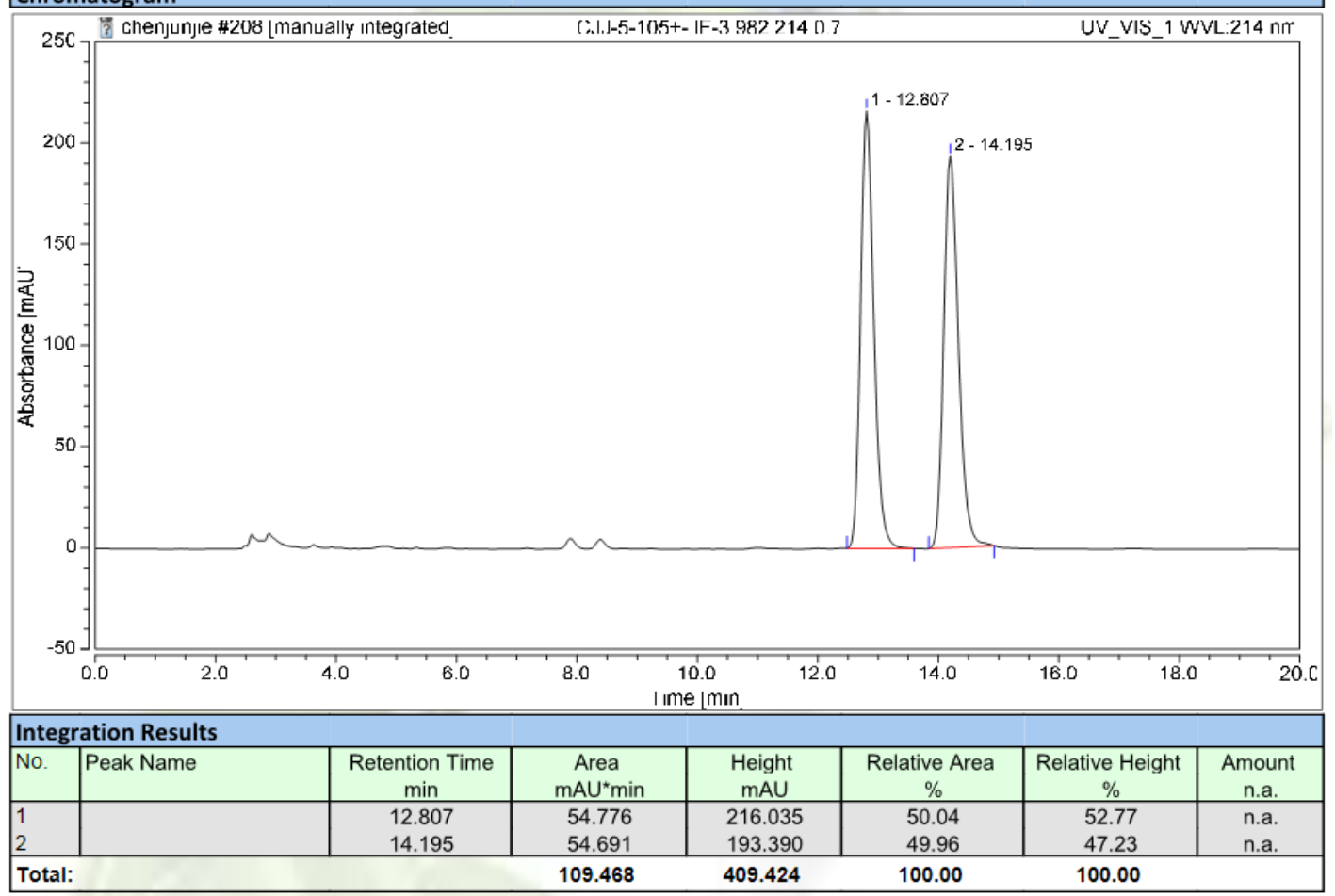

\section{Chromatogram}

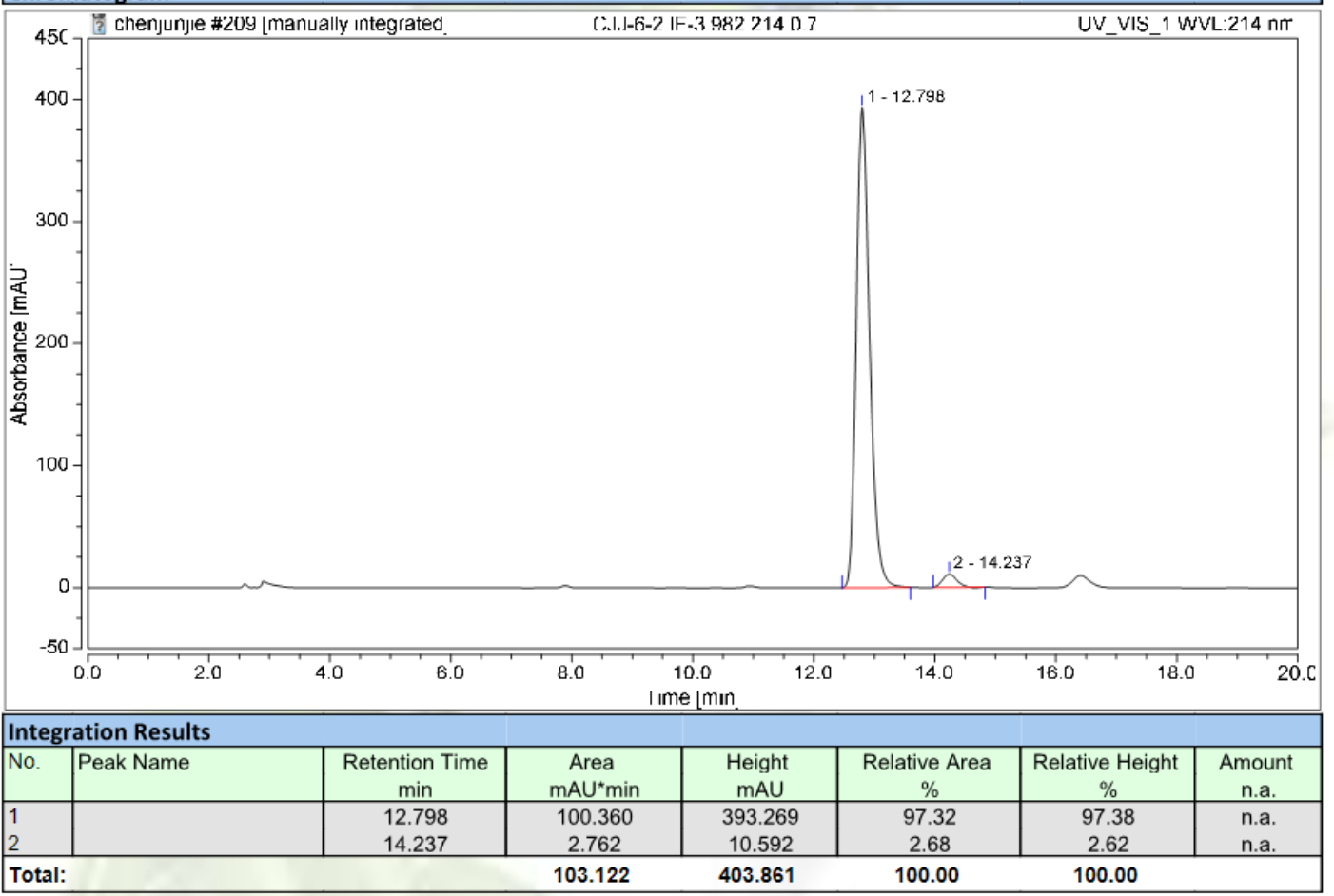




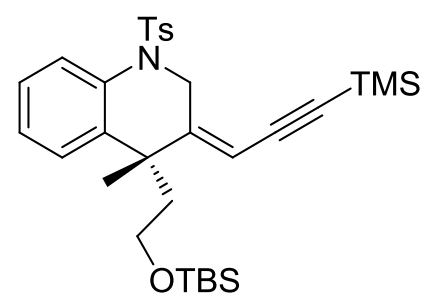

$7 a$

\section{Chromatogram}

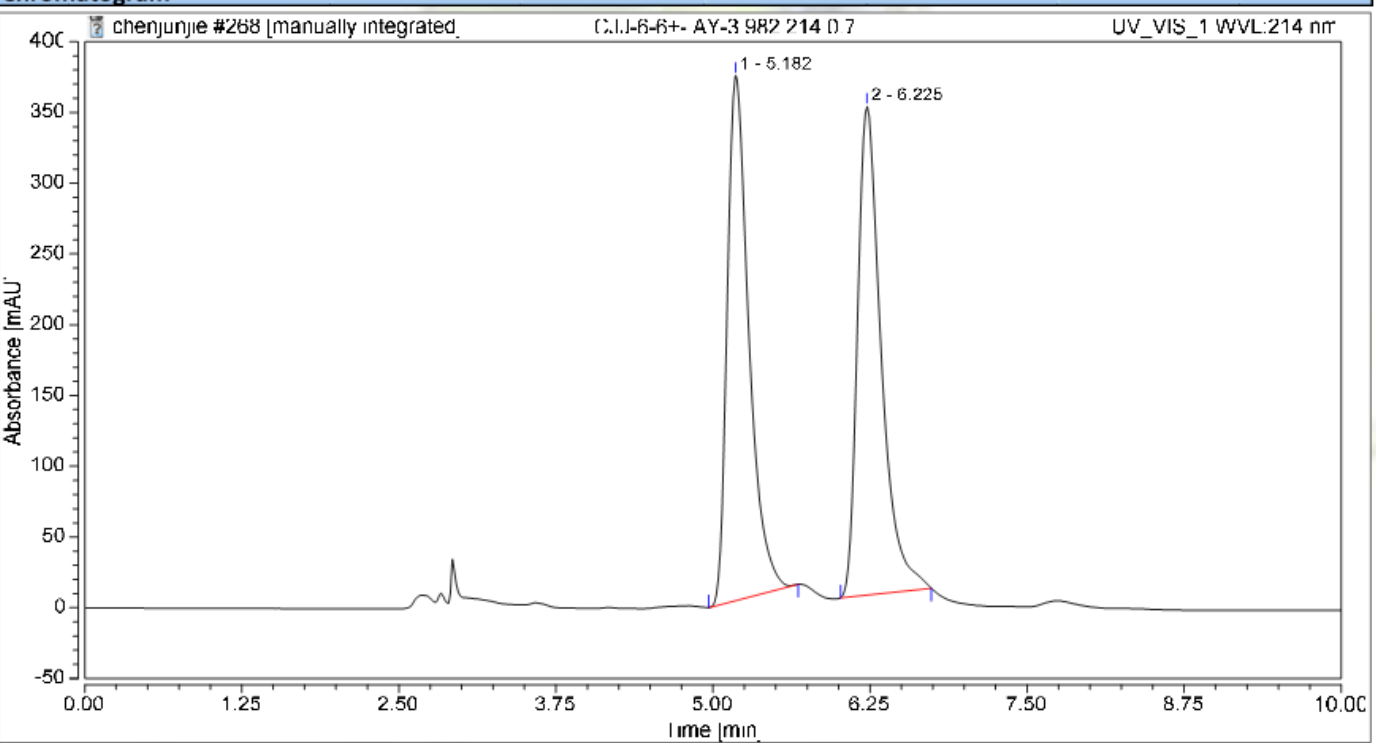

\begin{tabular}{|l|l|c|c|c|c|c|c|}
\hline \hline Integration Results \\
\hline No. & Peak Name & $\begin{array}{c}\text { Retention Time } \\
\text { min }\end{array}$ & $\begin{array}{c}\text { Area } \\
\mathrm{mAU} \text { min }\end{array}$ & $\begin{array}{c}\text { Height } \\
\mathrm{mAU}\end{array}$ & $\begin{array}{c}\text { Relative Area } \\
\%\end{array}$ & $\begin{array}{c}\text { Relative Height } \\
\%\end{array}$ & $\begin{array}{c}\text { Amount } \\
\text { n.a. }\end{array}$ \\
\hline 1 & & 5.182 & 73.769 & 371.089 & 49.36 & 51.79 & n.a. \\
2 & 6.225 & 75.675 & 345.479 & 50.64 & 48.21 & n.a. \\
\hline Total: & 149.444 & 716.568 & 100.00 & 100.00 \\
\hline
\end{tabular}

\section{Chromatogram}

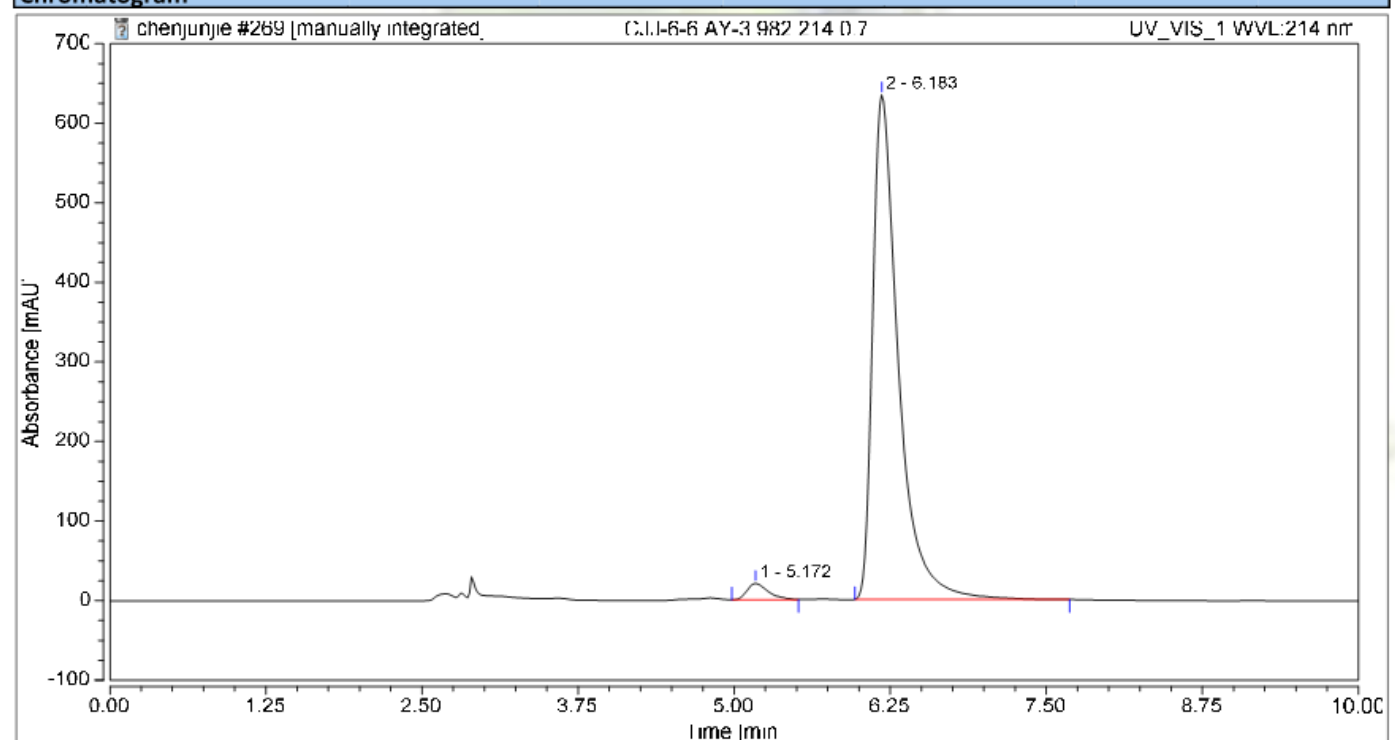

\begin{tabular}{|l|l|c|c|c|c|c|c|}
\hline \multicolumn{1}{|l|}{ Integration Results } \\
\hline No. & Peak Name & $\begin{array}{c}\text { Retention Time } \\
\text { min }\end{array}$ & $\begin{array}{c}\text { Area } \\
\text { mAU*min }\end{array}$ & $\begin{array}{c}\text { Height } \\
\text { mAU }\end{array}$ & $\begin{array}{c}\text { Relative Area } \\
\%\end{array}$ & $\begin{array}{c}\text { Relative Height } \\
\%\end{array}$ & $\begin{array}{c}\text { Amount } \\
\text { n.a. }\end{array}$ \\
\hline 1 & & 5.172 & 4.041 & 20.565 & 2.59 & 3.14 & n.a. \\
2 & 6.183 & 151.711 & 634.519 & 97.41 & 96.86 & n.a. \\
\hline
\end{tabular}




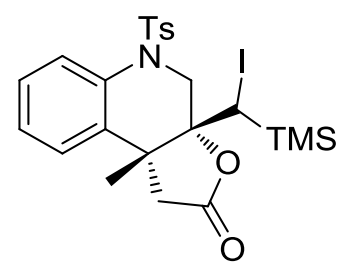

$8 a$
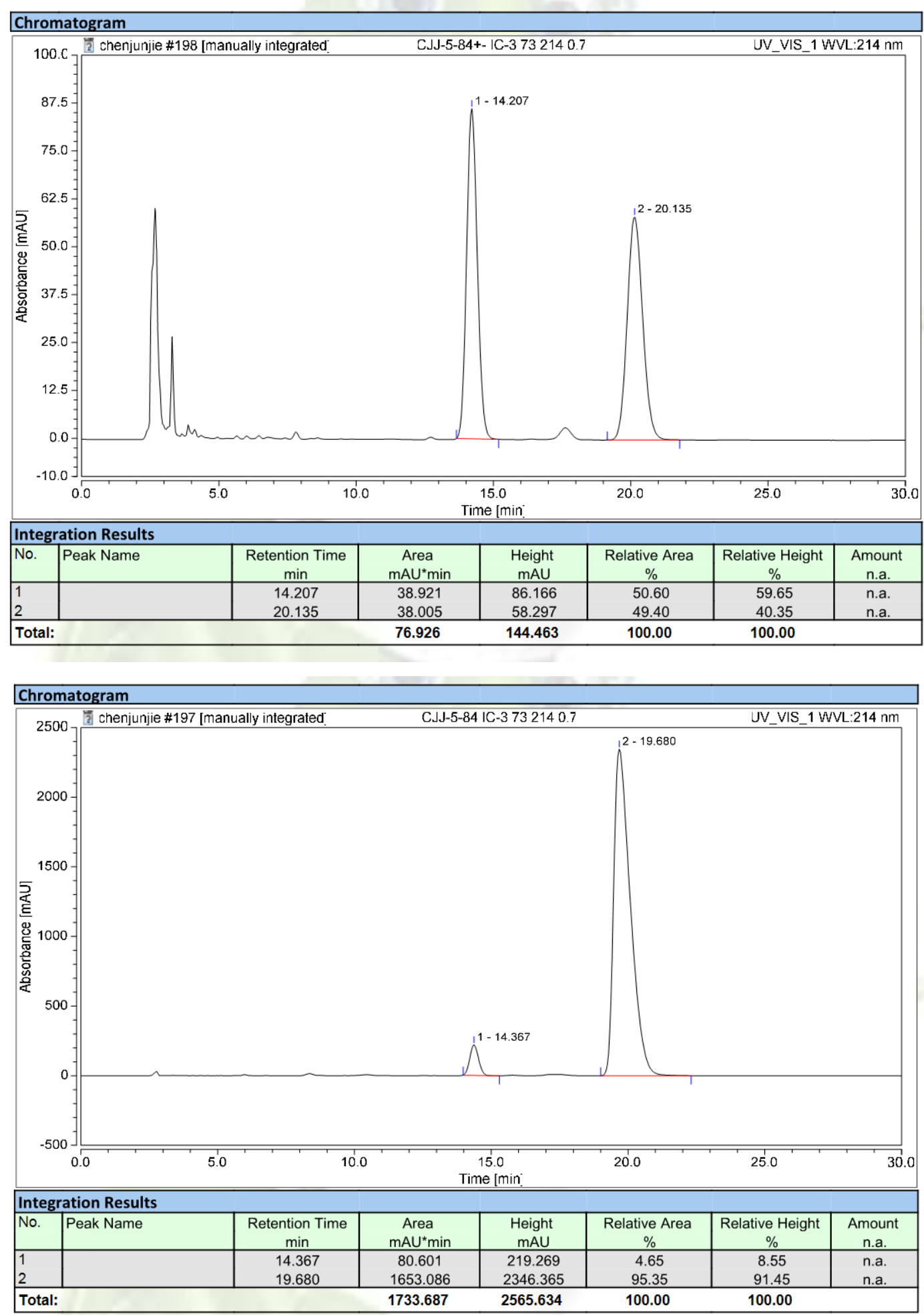
11. Crystal structure analyses for product 8a (CCDC 1919857).

The thermal ellipsoid drawn at 50\% probability level.

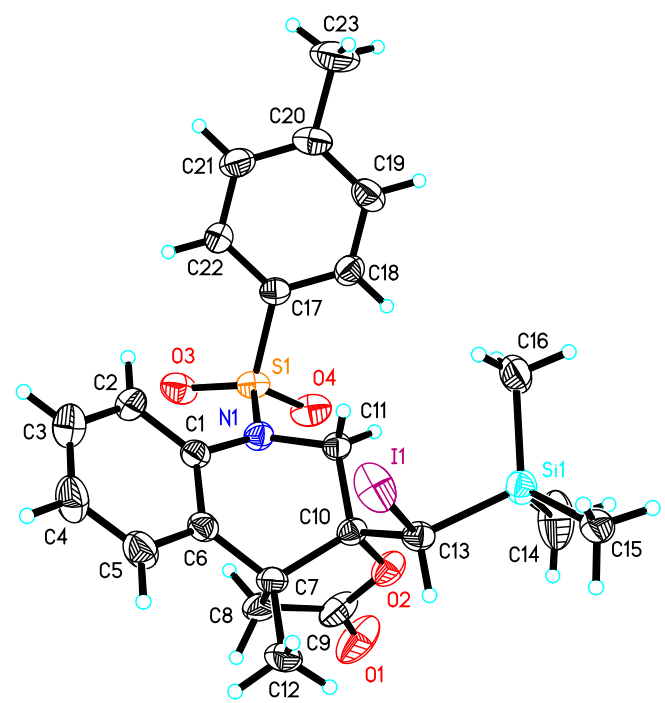

\section{Crystal data and structure refinement for $8 \mathrm{a}$}

Identification code

Empirical formula

Formula weight

Temperature

Wavelength

Crystal system

Space group

Unit cell dimensions

Volume

$\mathrm{Z}$

Density (calculated)

Absorption coefficient

$\mathrm{F}(000)$

Crystal size

Theta range for data collection

Index ranges

Reflections collected

Independent reflections

Completeness to theta $=25.242^{\circ}$ mo_d8v18611_0m

C23 H28 I N O4 S Si

569.51

296(2) K

$0.71073 \AA$

Orthorhombic

P 212121

$\mathrm{a}=11.9752(3) \AA \quad \alpha=90^{\circ}$.

$\mathrm{b}=13.1142(3) \AA \quad \beta=90^{\circ}$.

$\mathrm{c}=16.0811(4) \AA \quad \gamma=90^{\circ}$.

2525.46(11) $\AA^{3}$

4

$1.498 \mathrm{Mg} / \mathrm{m}^{3}$

$1.426 \mathrm{~mm}^{-1}$

1152

$0.180 \times 0.150 \times 0.100 \mathrm{~mm}^{3}$

2.972 to $25.999^{\circ}$.

$-13<=\mathrm{h}<=14,-16<=\mathrm{k}<=15,-19<=\mathrm{l}<=16$

14260

$4930[\mathrm{R}(\mathrm{int})=0.0262]$

$99.0 \%$ 
Absorption correction

Max. and min. transmission

Refinement method

Data / restraints / parameters

Goodness-of-fit on $\mathrm{F}^{2}$

Final $\mathrm{R}$ indices [I $>2 \operatorname{sigma}(\mathrm{I})]$

$\mathrm{R}$ indices (all data)

Absolute structure parameter

Extinction coefficient

Largest diff. peak and hole
Semi-empirical from equivalents

0.7456 and 0.5082

Full-matrix least-squares on $\mathrm{F}^{2}$

4930 / 0 / 286

1.031

$\mathrm{R} 1=0.0303, \mathrm{wR} 2=0.0724$

$\mathrm{R} 1=0.0371, \mathrm{wR} 2=0.0769$

$-0.010(8)$

$0.0106(11)$

0.604 and -0.594 e. $\AA^{-3}$ 